\title{
Topics in the theory of trusses
}

\author{
Bernard Rybołowicz
}

Submitted to Swansea University in fulfilment of the requirements for the Degree of Doctor of Philosophy

Department of Mathematics,

Swansea University,

2021

Supervisor: Prof. Tomasz Brzeziński

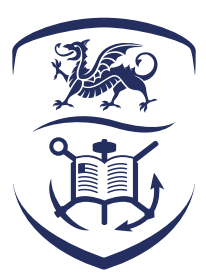

Swansea University

Prifysgol Abertawe

Copyright: The author, Bernard Rybolowicz, 2021. 


\section{Abstract}

In 2017 a truss was defined. Thus one can say that the theory of trusses is new and not yet well-established. In recent years trusses start to gain attention due to their connections to ring theory and braces. Braces are closely related to solutions of set-theoretic Yang-Baxter equations, which can lead to applications of trusses in physics. In this thesis, we study connections among groups, heaps, rings, modules, braces and trusses. In the beginning, one can find a description in details of free heaps and coproducts of Abelian heaps. Both constructions are applied to describe a functor from the category of heaps to the category of groups. We establish a connection between unital near-trusses and skew left braces. We show that for a specific choice of congruence on a unital near-truss the quotient is a brace. We also prove that if one localises a regular unital near-truss without an absorber, the result is a skew left brace. In this thesis, one can find many small results on categories of heaps, trusses and modules over a truss. Methods to extend trusses to unital trusses and rings are presented. Then first one allows us to show that a category of modules over a truss is isomorphic with the category of modules over its extension to the unital truss. The second method establishes a deep connection between rings and trusses, i.e. every truss is an equivalence class of some congruence on some specific ring. We present the ring construction. Using this result, we introduce the definition of a minimal extension of a truss into a ring. We construct tensor product and free modules over trusses. The Eilenberg-Watts theorem for modules over trusses is stated and proven. Thus the Morita theory for modules over trusses is developed. The thesis is concluded with results on projectivity and decompositions through a product of the modules. 


\section{Declarations and Statements}

\section{DECLARATION}

This work has not previously been accepted in substance for any degree and is not being concurrently submitted in candidature for any degree.

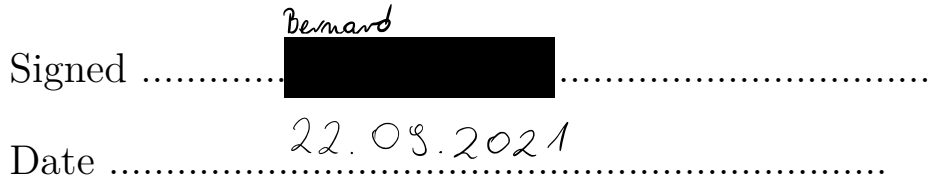

\section{STATEMENT 1}

This thesis is the result of my own investigations, except where otherwise stated. Where correction services have been used, the extent and nature of the correction is clearly marked in a footnote(s).

Other sources are acknowledged by footnotes giving explicit references. A bibliography is appended.

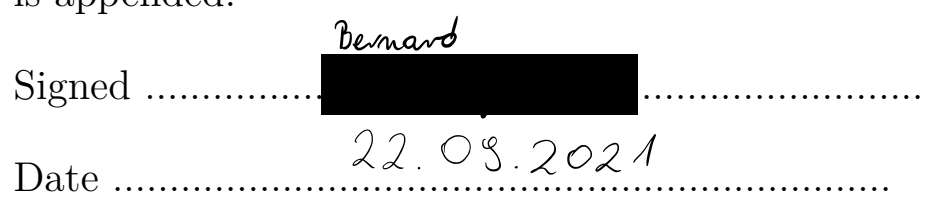

\section{STATEMENT 2}

I hereby give consent for my thesis, if accepted, to be available for photocopying and for inter-library loan, and for the title and summary to be made available to outside organisations.

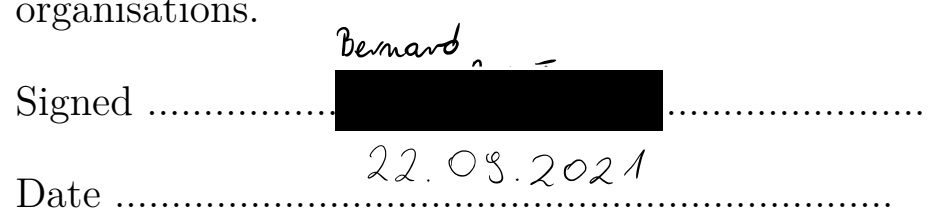




\section{Contents}

\begin{tabular}{ll}
\hline Introduction & 15
\end{tabular}

$\begin{array}{lll}\text { I Heaps } & 27\end{array}$

$\begin{array}{lll}1 & \text { In the world of heaps } & 29\end{array}$

1.1 Groups and heaps $\ldots \ldots \ldots \ldots$. . . . . . . . . . . . . . . 30

1.2 Quotient of a heap . . . . . . . . . . . . . . . . . . . . 40

1.3 Free heaps $\ldots \ldots \ldots \ldots \ldots \ldots$

1.4 Coproduct of Abelian heaps . . . . . . . . . . . . . . . . . . . . 51

1.5 Construction of a free group by heaps $\ldots \ldots \ldots \ldots \ldots$

II Trusses $\quad 63$

2 In the world of trusses $\quad 65$

$2.1 \quad$ Pre-trusses, near-trusses and trusses $\ldots \ldots \ldots \ldots \ldots$

2.2 Congruences on pre-trusses $\ldots \ldots \ldots \ldots \ldots$

2.3 Units from the perspective of trusses $\ldots \ldots \ldots \ldots \ldots 77$

$\begin{array}{lll}3 & \text { From trusses to braces } & 83\end{array}$

3.1 Near-trusses and braces . . . . . . . . . . . . . . . . . . . . . . . 83 
3.2 From a near-truss to a domain . . . . . . . . . . . . . . . . . . 88

3.3 A skew brace of fractions . . . . . . . . . . . . . . . . . . . 94

III Modules 105

\begin{tabular}{lll}
\hline In the world of modules & 107
\end{tabular}

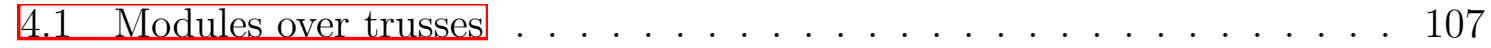

4.2 Congruences in modules . . . . . . . . . . . . . . . . . . . . 111

4.3 Coproduct of modules . . . . . . . . . . . . . . . . . . 117

4.4 Monomorphisms and epimorhpisms . . . . . . . . . . . . . . 118

$\begin{array}{lll}5 & \text { Extensions of trusses } & 123\end{array}$

5.1 On extensions of a truss . . . . . . . . . . . . . . . . . . . . 124

5.1 .1 Extension by a module . . . . . . . . . . . . . . . . . . . . . . . 124

5.1 .2 Coproduct extensions . . . . . . . . . . . . . . . . . . 133

5.2 Universal and minimal extensions to rings $\ldots \ldots \ldots$. . . . . . . . . 139

$5.2 .1 \quad$ Extending to a ring . . . . . . . . . . . . . . . . . 139

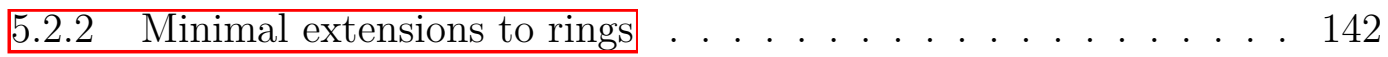

6 On the categories of modules $\quad 155$

6.1 Unital trusses and trusses $\ldots \ldots \ldots \ldots$. . . . . . . . . . 156

6.2 Free modules . . . . . . . . . . . . . . . . . . . . . . 158

6.3 Tensor product $\ldots \ldots \ldots$. . . . . . . . . . . . . . 166

$\begin{array}{lll}7 & \text { Morita theory and projective modules } & 181\end{array}$

7.1 Morita theory and modules over trusses . . . . . . . . . . . . . . . . . . 182 
$\begin{array}{ll}\text { CONTENTS } & 9\end{array}$

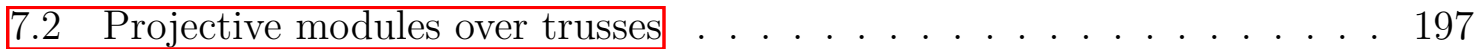

\begin{tabular}{ll}
\hline A Categories & 209
\end{tabular}

\begin{tabular}{ll}
\hline B Structures with binary operations & 217
\end{tabular}

B.1 Semigroups, monoids and groups . . . . . . . . . . . . . . . . 217

B.2 Near-rings, skew braces and braces . . . . . . . . . . . . . . . . . . . 220

\begin{tabular}{ll}
\hline C Universal algebra & 225
\end{tabular} 


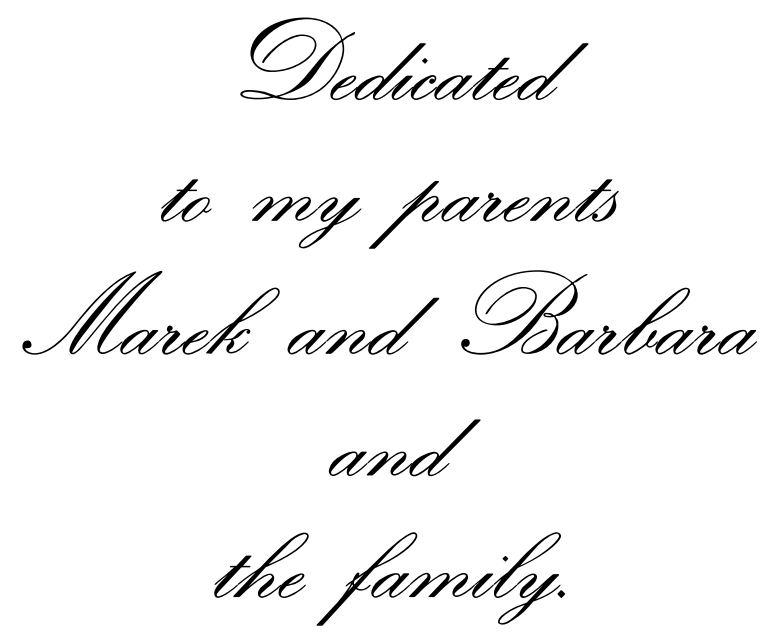




\section{Acknowledgements}

I wish to thank all my friends and family that always listened to my complaints and encouraged me to work. It was not an easy task, but thanks to you all, I finished it.

I wish to thank:

Tomasz Brzeziński, working with you for the last three years was a pleasure. Thank you for all the meaningful advice and enormous patience.

Stefano Mereta, for being my collaborator and lunch buddy but mainly for cookie breaks.

Paolo Saracco, for all the guidance and everything he taught me about category theory, for example, that one can prove something with a crazy big diagram that is too hard for me to follow.

My examiners Jeffrey Giansiracusa and Christian Lomp, for all the comments on the thesis.

Swansea University for funding my PhD project, all of this would not have been possible without the SURES scholarship.

All my teachers that I met until today at the University of Białystok and Swansea University. Thanks for being so enthusiastic about sharing your knowledge with me.

At last, I would like to thank anyone who will read or use this thesis in their work as only then it will fulfil its purpose. 


\section{Introduction}

The thesis is written to be as autonomous as possible. It consists of 3 parts: Heaps, Trusses and Modules. To understand the idea behind the truss theory one should be familiar with groups, rings, heaps and braces. First two are well-known by potential readers, so let us introduce heaps and braces.

\section{Heaps and groups}

In the 1920s, H. Prüfer [1], R. Baer [2] and A.K. Suskievic [3] introduced heaps. A heap is a set $H$ together with ternary operation $[-,-,-]: H \times H \times H \rightarrow H$ such that for all $a_{i} \in H$,

$$
\left[a_{1}, a_{2},\left[a_{3}, a_{4}, a_{5}\right]\right]=\left[\left[a_{1}, a_{2}, a_{3}\right], a_{4}, a_{5}\right] \text { and }\left[a_{1}, a_{1}, a_{2}\right]=\left[a_{2}, a_{1}, a_{1}\right]=a_{2}
$$

The first equality is called associativity, and the second one is called Mal'cev identities. There is a deep connection between heaps and groups. To every group $(G, \cdot)$ we can assign a heap $\mathrm{H}(G)$ in a functorial way by defining ternary operation by $[a, b, c]=a b^{-1} c$, for any $a, b, c \in G$. This heap is called a heap associated with group $G$. It is also possible to assign a group $\mathrm{G}(H ; e)$ to every heap $H$ and any $e \in H$. We achieve this by fixing the middle element in the ternary operation, i.e. $a \cdot{ }_{e} b=[a, e, b]$ is a group operation on $H$ with a neutral element $e$. This assignment is not functorial. To understand why, let us first introduce homomorphisms of heaps. A homomorphism of heaps is a map $f$ between heaps that preserves ternary operations, i.e. $f([a, b, c])=[f(a), f(b), f(c)]$. Every group homomorphism is a heap homomorphism of associated heaps. The opposite is not true. For example, if we consider a constant map from a group into itself, then that map is a group homomorphism if and only if the constant is the neutral element of the group. But it is always a homomorphism of associated heaps by Mal'cev identities. 
Thus heaps, in some sense, have more homomorphisms. Observe that lack of a unique choice of an element preserved by homomorphisms is the cause why the assignment of a group to a heap is not a functor. A sub-heap is a subset of a heap closed under the ternary operation. By preceding connection with groups, one can translate normality to heaps, see Definition 1.2.6. In [4, Theorem 1] J. Certaine (see Theorem 1.2.14) observed that since we do not have a particular choice of an element in a heap, there is a correspondence between sub-heaps and equivalence relation classes. Besides, any preimage of a single element of a heap homomorphism is a normal sub-heap. That implies two things, that we can take quotient by any congruence class in a heap and that the choice of a sub-heap is not unique, i.e. there exist more than one sub-heap such that the quotient is the same heap. All this sums up to a conclusion that a heap is an affinization of a group. We forget the neutral element of a group. That is not wrong since every heap gives a free transitive action on a set, and every free transitive action of a group on a set makes the set a heap.

Around 80 years later...

\section{Braces}

In $2007 \mathrm{~W}$. Rump in [5] introduced definition of a brace. A two-sided brace $B$ is a set with two group operations $+, \cdot: B \times B \rightarrow B$ such that for all $a, b, c \in B$,

$$
a+b=b+a, a(b+c)=a b-a+a c \text { and }(b+c) a=b a-a+c a .
$$

The second equality is called left distributivity, and the third is right distributivity. If only left distributivity holds, we say that $B$ is a left brace. In the same paper, W. Rump established connections among left braces, radical rings and non-degenerate involutive solutions of a set-theoretic Yang-Baxter equation. A map $R: X \times X \rightarrow X \times X$ is a solution of a set-theoretic Yang-Baxter equation if

$$
(R \times 1)(1 \times R)(R \times 1)=(1 \times R)(R \times 1)(1 \times R),
$$

where 1 is an identity map on $X$. To every set-theoretic solution, one can associate a solution of a quantum Yang-Baxter equation. Those solutions have applications 
in noncommutative geometry, see [6]. Since Rump paper appeared, the subject has been very intensively researched, e.g. [7], [8], [9], [10], [11], [12], [13], [14]. In 2017 L. Guarnieri and L. Vendramin introduced a method to produce a non-degenerate solution of a set-theoretic Yang-Baxter equation from a skew left brace, a left brace for which $a+b=b+a$ does not hold. We will omit the adjective left, and we will write skew brace for a skew left brace. The solution $R: B \times B \rightarrow B \times B$ associated with a skew brace $B$ is given for any $a, b \in B$ by

$$
R(a, b)=\left(-a+a b,(-a+a b)^{-1} a b\right)
$$

where $a^{-1}$ is an inverse of $a$ with respect to $\cdot$. We will focus on the algebraic properties of skew braces, omitting but keeping in mind their applications to quantum physics. A two-sided brace is an interesting algebraic object due to its similarity to fields. In both structures, we have two groups. The difference is that in a field, multiplication is not a group operation on a whole set as we do not have an inverse of zero. The left distributivity of a skew brace implies that neutral elements of both group operations are the same, i.e. $0=1$, and $0 \mathrm{a}=\mathrm{a}$, for all $a \in B$. One would suspect that there are only trivial congruences on braces, similarly to fields, but it is not correct. In contrast to the case of fields, one can consider non-trivial congruences on braces. The easiest example is to consider a group $(G,+)$, then $(G,+,+)$, i.e. $\cdot=+$, is a brace. All normal subgroups of $G$ are ideals in the sense of Definition B.2.29 in the brace $(G,+,+)$. One of the goals of this thesis is to study connections between unital near-trusses (See Definition 2.1.1) and skew braces. That will lead us to new constructions of skew braces, and therefore constructions of solutions of a set-theoretic Yang-Baxter equation.

Around 10 years after W. Rump introduced braces...

\section{Trusses}

In 2017 in a preprint of [15], T. Brzeziński introduced trusses. A truss is an Abelian heap $T$ with an additional associative binary operation $\cdot: T \times T \rightarrow T$, called multiplication, which distributes from both sides over the ternary operation, i.e. for all 
$a, b, c, d \in T$,

$$
a[b, c, d]=[a b, a c, a d] \&[b, c, d] a=[b a, c a, d a]
$$

The first equality is left distributivity, and the second is right distributivity. By the Abelian heap, we mean that $[a, b, c]=[c, b, a]$, that is, the heap associated with an Abelian group. A truss homomorphism is a map $f$ between trusses that preserves both operations, i.e. for all $a, b, c$,

$$
f([a, b, c])=[f(a), f(b), f(c)] \quad \& \quad f(a b)=f(a) f(b) .
$$

On every Abelian heap, we can build multiplication in at least two ways. First, commutative, choose a constant $c \in T$, then for all $a, b \in T, a b=c$. Second, noncommutative, for all $a, b \in T$ consider $a b=b$. Analogously one can define multiplication $b a=b$. Similarly to heaps, the first assignment is not a functor since we choose a constant, but the noncommutative assignment is functorial. The idea behind trusses is to unify both rings and braces in one distributive law of trusses. Therefore, with every ring $(R,+, \cdot)$, we can associate a truss $\mathrm{T}(R)=(\mathrm{H}(R), \cdot)$, i.e. we take a heap associated with an additive group of a ring $R$ and consider the same multiplication of a ring. This assignment $\mathrm{T}$ is a functor from the category of rings to the category of trusses since every ring homomorphism will preserve ternary operation. The interesting fact is that if we start with a truss and we have an element, called an absorber, $a \in T$ such that for all $b \in T, a b=b a=a$, then $a$ is unique and by taking retract $(\mathrm{G}(T ; a), \cdot)$, we get a ring. That is not a functor as homomorphisms of trusses do not necessarily preserve absorbers. But one can see that category of rings is isomorphic with some subcategory of trusses with an absorber. We choose all morphisms which preserve absorbers. The same reasoning leads to building analogous functor $\mathrm{T}$ from braces to unital trusses, i.e. trusses with an element, called identity, $1 \in T$ such that $1 a=a 1=a$. Unital trusses, which 1-retracts are braces, will be called brace-type or associated with braces. Since morphisms of unital trusses preserve identities, it will be a full subcategory. We see that rings and braces are trusses, but there are more, for example, truss with multiplication $a b=b$. Thus trusses provide us with the environment to study both rings and braces in a more general setting. Due to the affine nature of heaps, one can think of trusses 
as an affine version of rings and braces. Since we have this generalisation of rings, it is natural to consider modules over trusses. T. Brzeziński introduced modules over trusses in [16]. A module over a truss is a heap together with an action of truss by the endomorphisms of the heap. Two things motivate us to consider those modules. First, a module $M$ over a ring $R$ is a module $\mathrm{H}(M)$ over a truss $\mathrm{T}(R)$. Thus every vector space is a module over a truss. But not every module over a truss associated with a field is a vector space. Therefore, we have more objects when we consider modules over trusses. Second is the affine nature of $\mathrm{H}(M)$. As the thesis is write down, the second motivation is still a mystery to the author, and research on this matter continues.

There are three main results of this thesis:

- We establish a relationship of unital near-trusses and skew braces. In Proposition 3.1.5. Theorem 3.1.11, Corollary 3.3.5. Corollary 5.1.6, we present methods of acquiring new skew braces from near-trusses .

- In Proposition 5.1.11 and Proposition 5.1.10, we introduce new ways to extend trusses to unital trusses and trusses associated with rings. That leads to characterisation of extensions of trusses into rings by size, i.e. we introduce classes of locally small, small, and minimal extensions. See definitions 5.2.5, 5.2.18, 5.2.22, In Lemma 5.2.10, we have shown that every truss is a congruence class of some ring. The ring is in Proposition 5.1.10. In Remark 5.2.28, we point out possible further applications of minimal extensions to essential extensions in ring theory.

- The last main result is the Eilenberg-Watts theorem for trusses 7.1.3. That allows us to consider the Morita theory for trusses.

Even though there are three main results, many of the other lemmas and theorems in the thesis have their particular value and meaning. 


\section{Contents}

\section{Heaps}

\section{Chapter 1}

We start with an introduction to heaps. See Definition 1.1.1. Next, we describe a relationship between heaps and groups, i.e. we discuss the functor H : Grp $\rightarrow$ Heap, see Lemma 1.1.9 and Corollary 1.1.11, and $e$-retract $G(H ; e)$ for any heap $H$ and $e \in H$, see Lemma 1.1.12. To define a quotient heap, in Definition 1.2.6, we define a normal sub-heap. Further, in Corollary 1.2.15, we show a one-to-one correspondence between normal sub-heaps and all congruence classes of any retracted group. In Lemma 1.1.21. we introduce an importnat automorphism of heaps $\tau_{e}^{f}: H \rightarrow H$. For a particular choice of $e, f \in H$, restriction of $\tau_{e}^{f}$ to a normal sub-heap is the heap isomorphism between classes of the congruence. Let us fix a retract of $H$ and consider a normal subgroup $G$, then $\tau_{e}^{f}$ is the isomorphism of $e+G$ and $f+G$. See Lemma 1.2.13. In Section 1.3, we describe in detail the construction of free heaps. For any set $X$, $\mathrm{H}(\mathcal{G}(X)) \neq \mathcal{H}(X)$, i.e. that heap associated with a free group over a set $X$ is not the free heap over $X$, see Lemma 1.3.3. In Section 1.4, we construct a coproduct of Abelian heaps as the quotient of the free heap. By proposition 1.4.1, we managed to describe elements of a coproduct of two heaps by choosing two arbitrary elements, one of each coproduct component. That allows us to construct an isomorphism from Proposition 1.4 .4 between coproduct $A \boxplus B$ and $A \times B \times \mathbb{Z}$ for any heaps $A$ and $B$. Existence of coproduct for non-Abelian heaps is provided by the universal algebra as heaps form a variety of algebras, see Theorem C.0.16. In the non-Abelian case, the choice of relations generating the normal sub-heap of the free heap complicates due to the weaker associativity. Compare associativity in Definition 1.1.1 and equality (1.1.1). The assignment of a retract to a heap is not a functor. We cannot uniquely choose an element in every heap that homomorphisms preserve. In the last section, we use coproduct to extend heap by a singleton set, i.e. we take a coproduct with the terminal heap. The universal property of coproduct assures that the unique extension of heap homomorphism maps the singleton element of the extension into the singleton element 
of the extension of the codomain, see Lemma 1.5.1. Attaching the terminal heap with coproduct and taking retract in the element of the terminal heap is a functor see Lemma 1.5.4. Moreover, this functor is left adjoint to the functor $\mathrm{H}$, see Theorem 1.5.5. We conclude the chapter with an observation that the composition of the free heap functor and attachment of the terminal heap is a free group functor, Corollary 1.5.6, and we leave some thoughts on the free group construction.

\section{Trusses}

\section{Chapter 2}

In this chapter, we introduce a generalisation of a truss, called pre-truss. A pre-truss is a heap with an associative binary operation. If this operation distributes over the ternary operation from the left, the pre-truss is a near-truss. See Definition 2.1.1. In the same way, as braces and rings are examples of trusses, near-rings and skew braces are examples of near-trusses. See Lemma 2.1.9 and Lemma 2.1.11. For a quotient heap of a pre-truss to be a pre-truss, one needs to assume something more than just the normality of a sub-heap. We postulate that the normal sub-heap is a paragon that is all the congruence classes of heaps, and therefore normal sub-heaps of the congruence are closed, see Definition 2.2.1. Thus given any subset of a pre-truss, we can check if it is a congruence class. By Corollaries 2.2.8 and 2.2.11, there is a one-to-one correspondence between all congruences classes in skew braces (or near-rings) and paragons in the associated near-truss. Thus, we have an isomorphism of the category of skew braces and a full subcategory of unital near-trusses by Lemma 2.2.12. The invertible elements $U(R)$ of a ring $R$ never form a subring of $R$, as $0 \notin R$. But $U(R)$ is a subgroup of $(R, \cdot)$. Thus we can ask when $U(R)$ is a sub-heap of $\mathrm{T}(R)$, therefore a brace-type truss. In Proposition 2.3.2 and Theorem 2.3.5, we present some necessary conditions for a set of units to be a brace. In Corollary 2.3.6, we show that $U(\mathbb{Z} / n \mathbb{Z})$ is a brace if and only if $n=2^{k}$ for any $k \in \mathbb{N}$. The Abelian cyclic brace is a commutative brace with the underlying cyclic additive group. We conclude the chapter with Proposition 2.3.9 with a derivation of Abelian cyclic braces of the form $\mathbb{Z} / 2^{m} \mathbb{Z}$ as a quotient of a commutative truss by a paragon. 


\section{Chapter 3}

In this chapter, we focus on the relationship between near-trusses and skew braces. We show that a near-truss $T$ with exactly one left ideal, i.e. a normal sub-heap $I$ such that for all $t \in T$ and $i \in I, t i \in I$, is a skew brace, see Proposition 3.1.5. That leads to a question when the quotient of a truss has only one left ideal. The answer is Theorem 3.1.11. Sadly, the condition given in this theorem is not practical. The next goal is to localise near-trusses to skew braces. To perform localisation, one need to consider regular near-trusses (Definition 3.3.1), i.e. domains with the Ore conditions. A domain is a near-truss $T$ in which right and left cancellation properties hold. Cancellation properties state that for all $a, b, c \in T, a b=a c \Longrightarrow b=c$ and $b a=c a \Longrightarrow b=c$. See Definition 3.2.5 and Lemma 3.2.6. We show that one can acquire a domain from a truss $T$ as the quotient by a completely prime paragon, see Definition 3.2.7 and Lemma 3.2.11. We conclude the chapter with a detailed description of localisation in Theorem 3.3.2 and Corollary 3.3.5, which states that the localisation of a near-truss without an absorber is a skew brace.

\section{Chapter 4}

In this chapter, we introduce the basics of modules over trusses. This chapter is technical. Its main aim is to familiarise the reader with modules over trusses and develop tools for further chapters. In Definition 4.2.2 and Lemmalem:induced action, one can find the definition of an induced action and an induced module. As in heaps and trusses, induced submodules correspond to congruence classes. See Theorem 4.2.13. As in the previous structures, a module $M$ over a ring $R$ is a module $\mathrm{H}(M)$ over a truss $\mathrm{T}(R)$ with the action given by the action of $R$ on $M$. Not every module $M$ over a truss $\mathrm{T}(R)$ is a module over a ring. A heap $M$ is a $\mathrm{T}(R)$-module with the action given for all $r \in R$ and $m \in M$ by $r m=m$. That is not a module over a ring $R$. Thus, modules over a truss associated with a ring are more general than modules over rings. In Section 4.3, we show that a coproduct of $T$-modules, as heaps, can be endowed with $T$-action, which makes it a coproduct of $T$-modules. We conclude this chapter with some results on monomorphisms and epimorphisms, e.g. Proposition 4.4.1. Proposition 
4.4.2. Lemma 4.4.6.

\section{Modules}

\section{Chapter 5}

In this chapter, we consider extensions of trusses, mainly into braces and rings. For a truss $T$ and a $T$-module $M$, we can define a truss on the product $T \times M$, see Theorem 5.1.2. This construction gives us a truss associated with a ring if and only if the module is a singleton set and truss $T$ is associated with a ring, see Lemma 5.1.3. In Corollary 5.1.6, we show that the extension by a module is a brace if and only if the truss is brace-type and the module is unital. Thus any unital module over a truss associated with a brace can be extended to a brace. In Remark 5.1.6, we observe that due to the construction of the extension, we can drop the right distributivity of $T$. The coproduct of a truss $T$ with a singleton $T$-module can be equipped with two truss multiplications. First makes a singleton element an identity. See Proposition 5.1.11. Thus the extended truss is unital. The second makes a singleton element an absorber. See Proposition 5.1.10. Thus the extended truss is associated with a ring. Attaching an absorber has a particular universal property. Any truss homomorphism from a truss to a truss associated with a ring uniquely extends to a ring homomorphism from a retract of a truss with an attached absorber $\mathrm{R}_{0}(T)=(\mathrm{G}(T \boxplus 0 ; 0), \cdot)$, see Lemma 5.2.1. This universal property ensures that $R_{0}$ is a functor from the category of trusses to the category of rings, which is a left adjoint to T, see Proposition 5.2.4. Let us consider truss $T$ and any ring extension $R$, i.e. we have injective homomorphism of trusses $\varphi: T \rightarrow \mathrm{T}(R)$. By the universal property of the extension by an absorber, we have that $\varphi$ uniquely extends to $\widehat{\varphi}: \mathrm{R}_{0}(T) \rightarrow R$. Thus, for any ring extension of $T$, we can find an ideal in $\mathrm{R}_{0}(T)$, namely $\operatorname{ker}(\widehat{\varphi})$. That allows us to introduce criterium of size to extensions into rings. A locally small extension of a truss $T$ is a ring $R$ such that $\mathrm{R}_{0}(T) / I \cong R$ for some ideal $I$. See definition 5.2.5 and Proposition 5.2.6. An image $\iota_{T}(T)$ of a canonical injection into $\mathrm{R}_{0}(T)$ is a paragon in $\mathrm{R}_{0}(T)$. See Lemma 5.2.10. Thus we can choose an ideal $\mathrm{I}(T)$, see Remark 5.2.11, for which $\iota_{T}(T)$ is a congruence class. That allows us to introduce a small extension as an extension in which $\widehat{\varphi}(\mathrm{I}(T))$ 
is essential, see Definition B.2.10. A small extension is not unique. For some trusses, there exists a particular universal choice of a small extension. This extension is called minimal. See Definition 5.2.22. We conclude this chapter with Remark 5.2.28, which points out possible future applications of minimal extensions to ring theory.

\section{Chapter 6}

In this chapter, we focus on categories of modules over trusses. Consider the extension of a truss to a unital truss from the previous chapter. Then the category of modules over a truss and the category of modules over the truss extended by the identity are isomorphic. See Theorem 6.1.2. Keeping in mind the isomorphism, we introduce a free $T$-module for a unital truss $T$ as a coproduct of $T$ 's, see Definition 6.2.2. In Lemma 6.2.4 we show that freeness is equivalent for a module to admit the basis. Since there exist modules over $\mathrm{T}(R)$ that are not modules over a ring $R$, one expects that free modules over rings are not free over trusses associated with them. The difference is in the number of absorbers, i.e. elements $m$ of the module such that for all $t \in T, t m=m$. Thus we introduce a functor $(-)_{\mathrm{Abs}}: \mathrm{T}(R)$-mod $\rightarrow R$-mod, which glues all the absorbers together. This functor is a left adjoint to the functor T, which assigns to every $R$-module a $\mathrm{T}(R)$-module. See Lemma 6.2.6. If $M$ is a free $\mathrm{T}(R)$-module with a basis $B$, then $M_{\mathrm{Abs}}$ is a free $R$-module with the same basis, see Theorem 6.2.7. The problem which highlights the difficulty of dealing with trusses without an absorber is to prove that a free $T$-module, for a truss $T$ without an absorber, has no absorbers. It is confirmed in Corollary 7.2.12 of Chapter 7 using projectivity. In Theorem 6.3.4, we introduce the tensor product of modules over trusses. In the rest of the chapter, we prove the basic properties of the tensor product, analogous to the tensor product of rings. For example, tensoring $-\otimes M$ is a left adjoint to $\operatorname{Hom}_{T}(M,-)$, Proposition 6.3.9 the free module with basis $X$ is isomorphic to the tensor product of heaps $T \otimes \mathcal{A}(X)$, of a truss $T$ and a free heap over $X, \mathcal{A}(X)$, see Proposition 6.3.13. We conclude the chapter with the observation that every module is a quotient of a free one. 


\section{Chapter 7}

In the last chapter, we develop the Morita theory for trusses. We say that two rings $R, S$ are Morita equivalent if there is an equivalence of their left module categories. The crucial tool of Morita theory is the Eilenberg-Watts theorem, which identifies all cocontinuous functors $\mathrm{F}: R$-mod $\rightarrow S$-mod, i.e. functors which preserve small colimits, with tensoring by some $S, R$-bimodule $M$, i.e. $F \cong M \otimes_{R}-$, naturally. We present the analogous theorem for trusses, see Eilenberg-Watts theorem for trusses 7.1.3. In the modules over rings, $\mathrm{F}$ is an equivalence if $M$ is projective and finitely generated. Then $\operatorname{Hom}_{S}(M,-)$ is the other functor of the equivalence. Two rings $R$ and $S$ are Morita equivalent if and only if $S \cong \operatorname{End}_{R}(P)$, for some finitely generated projective $R$-module $P$. The crucial part of the proof uses the fact that the dual module $\operatorname{Hom}_{R}(P, R)$ admits a dual basis. For trusses, it is not clear when $\operatorname{Hom}_{T}(P, T)$ fulfils condition similar to the existence of a dual basis. We introduce this property in Definition 7.1.8 and name it a dual basis property. Modules satisfying dual basis property are called tiny. See Definition 7.1.12 and Theorem 7.1.11. Observe that by Theorem 7.1.11, $\operatorname{Hom}_{S}(M,-)$ preserves colimits if and only if $\mathrm{M}$ is tiny, so for $\mathrm{F}$ to be equivalence, $M$ must be tiny. This theory coincides with the Morita theory for rings as every projective finitely generated $R$ module is tiny in the sense of the $\mathrm{T}(R)$-module. In contrast to the theory of rings, in this case, free $T$-modules are not tiny. In Proposition 7.2 .8 , we show that every tiny module is projective and finitely generated. Next, we consider splittings of short exact sequences. Since the category of $T$-modules is not Abelian, it is not obvious how to define an exact sequence. Thus we introduce the definition at the beginning of Section 7.2. In Proposition 7.2.2 and Proposition 7.2.5. we observe that the existence of section (retraction) of a sequence

$$
0 \longrightarrow \mathrm{G}\left(M ; e^{\prime}\right) \stackrel{\phi}{\longrightarrow} \mathrm{G}(N ; \underbrace{\sigma(e)) \stackrel{\psi}{\longrightarrow}}_{\sigma} \mathrm{G}(P ; e) \longrightarrow 0 .
$$

leads to a decomposition of the module $N$ into $M \times P \cong N\left(M^{(e)} \times P \cong N\right)$ as $T$-modules. We conclude this chapter with different results on a decomposition of modules. By Theorem 7.2.18, $T$-module $M$ is projective if and only if $M$ is a direct 
factor of free $T$-module such that the complementary factor is a module with the absorber. In Proposition 7.2.19, we observe that every tiny module is a factor of a $T$-module $T^{s}=T \times \ldots \times T$.

\section{Appendix A}

This appendix contains basic definitions and theorems on category theory.

Appendix $B$

This appendix contains basic definitions and theorems on groups, near-rings and skew braces.

\section{Appendix $C$}

This appendix contains basic definitions and theorems on universal algebras.

\section{Enjoy the thesis.}




\section{Part I}

\section{Heaps}





\section{Chapter 1}

\section{In the world of heaps}

Heaps were defined in the 1920s by H. Prüfer (see [1]) and R. Baer (see [2]) as an algebraic system consisting of a set with a ternary operation which fulfils conditions called associativity and Mal'cev identities, see Definition 1.1.1. This chapter is devoted to heaps, as heaps are for trusses the same as groups are for rings. All the categories discussed in this section, that is categories of heaps, Abelian heaps, groups and Abelian groups are varieties of algebras (in the sense of the universal algebra), hence they have free objects, limits, coproducts, euqalisers, coequalisers etc., see appendices $\mathrm{A}$ and C or [17] and [18]. The aim of this section is to give explicit constructions of free heaps, free Abelian heaps, coequalisers of heaps and coproduct of Abelian heaps. The complementary literature for this chapter is [16], [19], [20] and [21].

First and second sections consist of basic definitions and properties of heaps and quotient heaps.

In the third section a construction of a free heap is described in details. Fourth contain a construction of a coproduct of Abelian heaps. Third and fourth sections are a third section from [19.

In the fifth section we present a new construction of a free groups through construction of free heaps by constructing a left adjoint functor to the functor $\mathrm{H}$ between category of groups and heaps. This functor allows us to decompose a free group functor through the category of heaps. This highlights how identity and inverses naturally 
appears in the free construction of a free group. Fifth section is based on [22].

\subsection{Groups and heaps}

The aim of this section is to introduce reader with the term of a heap and describe connection between heaps and groups.

Definition 1.1.1. A heap is a set $H$ together with a ternary operation,

$$
[-,-,-]: H \times H \times H \longrightarrow H
$$

such that, for all $a_{1}, a_{2}, a_{3}, a_{4}, a_{5} \in H$,

(1) $\left[a_{1}, a_{2},\left[a_{3}, a_{4}, a_{5}\right]\right]=\left[\left[a_{1}, a_{2}, a_{3}\right], a_{4}, a_{5}\right]$, (Associativity)

(2) $\left[a_{1}, a_{1}, a_{2}\right]=a_{2}=\left[a_{2}, a_{1}, a_{1}\right]$. (Mal'cev identities)

We say that $H$ is an Abelian heap if, for all $a, b, c \in H,[a, b, c]=[c, b, a]$.

Definition 1.1.2. Let $H_{1}$ and $H_{2}$ be heaps. A map $f: H_{1} \longrightarrow H_{2}$ is a heap homomorphism if, for all $a, b, c \in H_{1}$,

$$
f([a, b, c])=[f(a), f(b), f(c)]
$$

Example 1.1.3. The integer numbers $\mathbb{Z}$ with the ternary operation $[-,-,-]$ given by $[k, l, m]:=k-l+m$, for all $k, l, m \in \mathbb{Z}$, form a heap.

Example 1.1.4. A set of odd integer numbers $2 \mathbb{Z}+1:=\{2 k+1 \mid k \in \mathbb{Z}\}$ with the ternary operation given by $[k, l, m]:=k-l+m$ is a heap.

Corollary 1.1.5. Heaps as objects and heap homomorphisms as arrows form a category, which is denoted by Heap. The full subcategory of the category of heaps consisting of only Abelian heaps is denoted by Ah.

Proof. Observe that since every heap homomorphism is a function between underlying sets it is enough to check that a composition of heap homomorphisms is a heap 
homomorphism. Let $f: H_{1} \rightarrow H_{2}$ and $g: H_{2} \rightarrow H_{3}$ be a heap homomorphisms among heaps $H_{1}, H_{2}$ and $H_{3}$, then for all $a, b, c \in H_{1}$,

$$
g \circ f([a, b, c])=g([f(a), f(b), f(c)])=[g \circ f(a), g \circ f(b), g \circ f(c)]
$$

Therefore composition of heap homomorphisms is a heap homomorphism, so Heap and $\mathbf{A} \mathbf{h}$ are categories. By the definition of a heap homomorphism $\mathbf{A} \mathbf{h}\left(H_{1}, H_{2}\right)=$ $\mathbf{H e a p}\left(H_{1}, H_{2}\right)$, therefore $\mathbf{A h}$ is a full subcategory of Heap.

Remark 1.1.6. A singleton set with the (unique) ternary operation is the terminal object in categories Heap and Ah, we denote this heap by $\{*\}$.

Remark 1.1.7. As the definition of a heap uses only universal quantifiers, the empty set with the unique ternary operation given by $\emptyset \times \emptyset \times \emptyset \longrightarrow \emptyset$ is a heap, which is the initial object in categories Heap and Ah.

Lemma 1.1.8. Let $\varphi \in \operatorname{Heap}\left(H_{1}, H_{2}\right)$, then

(1) $\varphi$ is a monomorphism if and only if $\varphi$ is injective,

(2) surjectivity of $\varphi$ implies that $\varphi$ is an epimorphism.

Proof. First observe that every homomorphism of heaps is a function between underlying sets, thus every injective (surjective) homomorphism is a monomorphism (an epimorphism), respectively. Now, assume that $\varphi: H_{1} \rightarrow H_{2}$ is a monomorphism and $r, z \in H_{1}, \varphi(r)=\varphi(z)$, then for any heap $H_{3}$ we can consider two heap homomorphisms $f_{1}, f_{2}: H_{3} \rightarrow H_{1}$ given by $f_{1}(h)=r$ and $f_{2}(h)=z$ for all $h \in H_{3}$. Since $\varphi$ is a monomorphism and $\varphi \circ f_{1}=\varphi \circ f_{2}$ we get that $f_{1}=f_{2}$. Thus $r=z$ and $\varphi$ is an injection.

Lemma 1.1.9. Let $G$ be a group. Then a pair $(G,[-,-,-])$, where $[a, b, c]:=a b^{-1} c$ for all $a, b, c \in G$, is a heap. This heap will be called a heap associated with a group $G$ and denoted by $\mathrm{H}(G)$. Moreover if $f: G \rightarrow G^{\prime}$ is a homomorphism of groups, then a map $\mathrm{H}(f): \mathrm{H}(G) \rightarrow \mathrm{H}\left(G^{\prime}\right)$ given by $\mathrm{H}(f)(x)=f(x)$, for all $x \in \mathrm{H}(G)$, is a heap homomorphism. 
Proof. The ternary operation is well-defined as it is given by the group operation and inverse, both of which are well-defined on $G$. Let $a, b, c, d, e \in G$, then

$$
\begin{gathered}
{[[a, b, c], d, e]=\left[a b^{-1} c, d, e\right]=a b^{-1} c d^{-1} e=\left[a, b, c d^{-1} e\right]=[a, b,[c, d, e]]} \\
{[a, a, b]=a a^{-1} b=b=b a^{-1} a=[b, a, a]}
\end{gathered}
$$

Thus $(G,[-,-,-])$ is a heap. Now, if $f: G \rightarrow G^{\prime}$ is a group homomorphism then for all $a, b, c \in \mathrm{H}(G)$,

$$
\mathrm{H}(f)([a, b, c])=f\left(a b^{-1} c\right)=f(a) f(b)^{-1} f(c)=[\mathrm{H}(f)(a), \mathrm{H}(f)(b), \mathrm{H}(f)(c)] .
$$

Therefore $\mathrm{H}(f)$ is a heap homomorphism.

Corollary 1.1.10. Let $G$ be a group. Then $G$ is an Abelian group if and only if $\mathrm{H}(G)$ is an Abelian heap.

Proof. Let $G$ be Abelian. Then for all $a, b, c \in G$

$$
[a, b, c]=a b^{-1} c=c b^{-1} a=[c, b, a]
$$

so $\mathrm{H}(G)$ is Abelian. Now, Assume $\mathrm{H}(G)$ is Abelian, then for all $a, b \in G$ and neutral element $e \in G$,

$$
a b=a e^{-1} b=[a, e, b]=[b, e, a]=b e^{-1} a=b a .
$$

Thus, $G$ is Abelian.

Corollary 1.1.11. The assignment $\mathrm{H}:$ Grp $\rightarrow$ Heap of a heap to a group, defined as in Lemma 1.1.9, is a functor. Moreover, the restriction $\mathrm{H}_{\mathbf{A b}}: \mathbf{A b} \rightarrow \mathbf{A h}$ of a functor $\mathrm{H}$ to Abelian groups is a functor between Abelian groups and Abelian heaps.

Proof. Let $G, G^{\prime}, G^{\prime \prime}$ be groups and $f: G \rightarrow G^{\prime}, g: G^{\prime} \rightarrow G^{\prime \prime}$ be group homomorphisms, then

$$
\mathrm{H}(g \circ f)=g \circ f=\mathrm{H}(g) \circ \mathrm{H}(f)
$$


by the definition of $\mathrm{H}$. For an identity homomorphism $1_{G}: G \rightarrow G$ and all $x \in \mathrm{H}(G)$, $\mathrm{H}\left(1_{G}\right)(x)=1_{G}(x)=x$, so $\mathrm{H}\left(1_{G}\right)=1_{\mathrm{H}(G)}$. Thus $\mathrm{H}$ is a functor. Second statement follows by Corollary 1.1 .10 .

Lemma 1.1.12. Let $H$ be a heap. Then for all $e \in H$ a pair $\left(H,+_{e}\right)$, where $+_{e}:=$ $[-, e,-]: H \times H \rightarrow H$, is a group. The group will be denoted by $\mathrm{G}(H ; e)$ and called a retract of $H$ in $e$ or an e-retract of $H$.

Proof. The operation $+_{e}$ is a well-defined binary operation, since $[-,-,-]$ is a welldefined ternary operation. Associativity follows by the associativity of ternary operation. Now, $e$ is a neutral element as for all $a \in H$,

$$
e+{ }_{e} a=[e, e, a]=a=[a, e, e]=a+{ }_{e} e,
$$

where second and third equalities follows by Mal'cev identities. The inverse to an element $a \in H$ is $[e, a, e]$, indeed as

$$
\begin{gathered}
a+{ }_{e}[e, a, e]=[a, e,[e, a, e]]=[[a, e, e] a, e]=[a, a, e]=e, \\
{[e, a, e]+{ }_{e} a=[[e, a, e], e, a]=[e, a,[e, e, a]]=[e, a, a]=e,}
\end{gathered}
$$

where in both cases the second equality follows by associativity and the third by Mal'cev identities. Therefore $\mathrm{G}(H ; e)$ is a group.

Corollary 1.1.13. A heap homomorphism $f: H \rightarrow H^{\prime}$ is a group homomorphism between retracts $\mathrm{G}(H ; e)$ and $\mathrm{G}\left(H ; e^{\prime}\right)$ if and only if $f(e)=e^{\prime}$.

The following corollary is a special case of Proposition 4.4.1. We present another proof using the fact that every group epimorphism is surjective.

Corollary 1.1.14. If $f$ is a heap epimorphism, then $f$ is surjective.

Proof. Let $f: H \rightarrow H^{\prime}$ be a heap epimorphism. We will show that $f$ is an epimorphism of groups. Let $e \in H$, then $f: \mathrm{G}(H ; e) \rightarrow \mathrm{G}\left(H^{\prime} ; f(e)\right)$ is a group homomorphism. Moreover, for any group homomorphisms $g, h: \mathrm{G}\left(H^{\prime} ; f(e)\right) \rightarrow G$ such that $g \circ f=h \circ f$, 
$\mathrm{H}(g) \circ f=\mathrm{H}(h) \circ f$, and since $f$ is an epimorphism of heaps we get that $\mathrm{H}(g)=\mathrm{H}(h)$. Thus, since $\mathrm{H}(g)=g$ and $\mathrm{H}(h)=h$ as functions, we get that $g=h$. Therefore $f$ is an epimorphism of groups and in consequence it is surjective, see B.1.4.

Example 1.1.15. A 1-retract $G(2 \mathbb{Z}+1 ; 1)$ is isomorphic to $\mathbb{Z}$.

Remark 1.1.16. In contrast to the assignment $\mathrm{H}$ of a heap to a group, the assignment of a group to a heap $H \mapsto \mathrm{G}(H ; e)$ is not a functor as it depends on the element $e \in H$, and therefore not every heap homomorphism can be interpreted as a group homomorphism of specific retracts, so the assignment is not a well-defined functor on morphisms.

Remark 1.1.17. The category of heaps is equivalent to a category of pointed heaps, i.e. a category of which objects are pairs of a heap and its element and homomorphism are homomorphisms between heaps which map a chosen element into a chosen element.

Corollary 1.1.18. Let $H$ be a heap and $e \in H$, then

$$
\mathrm{H}(\mathrm{G}(H ; e))=H .
$$

Proof. As underlying sets of $\mathrm{H}(\mathrm{G}(H ; e))$ and $H$ are the same, the statement follows by the following equality,

$$
a_{1}+{ }_{e} a_{2}^{-1}+{ }_{e} a_{3}=\left[\left[a_{1}, e, a_{2}^{-1}\right], e, a_{3}\right]=\left[\left[a_{1}, e,\left[e, a_{2}, e\right]\right], e, a_{3}\right]=\left[a_{1}, a_{2}, a_{3}\right]
$$

Lemma 1.1.19. If $H$ is a heap then for all $a_{1}, a_{2}, a_{3}, a_{4}, a_{5} \in H$,

(1) $\left[\left[a_{1}, a_{2}, a_{3}\right], a_{4}, a_{5}\right]=\left[a_{1},\left[a_{4}, a_{3}, a_{2}\right], a_{5}\right]$,

(2) $\left[a_{1}, a_{1}, a_{1}\right]=a_{1}$,

(3) $\left[a_{1}, a_{2}, a_{3}\right]=a_{1} \Longleftrightarrow a_{2}=a_{3}$,

(4) $\left[\left[a_{1}, a_{2}, a_{3}\right], a_{4}, a_{5}\right]=\left[\left[a_{1}, a_{4}, a_{5}\right],\left[a_{2}, a_{4}, a_{5}\right],\left[a_{3}, a_{4}, a_{5}\right]\right]$.

Proof. (1) Let $e, a_{1}, a_{2}, a_{3}, a_{4}, a_{5} \in H$, then

$$
\left[\left[a_{1}, a_{2}, a_{3}\right], a_{4}, a_{5}\right]=a_{1}+{ }_{e} a_{2}^{-1}+{ }_{e} a_{3}+{ }_{e} a_{4}^{-1}+{ }_{e} a_{5}
$$


in the $e$-retract $\mathrm{G}(H ; e)$. On the other hand side

$$
\left[a_{1},\left[a_{4}, a_{3}, a_{2}\right], a_{5}\right]=a_{1}+{ }_{e}\left(a_{4}+{ }_{e} a_{3}^{-1}+_{e} a_{2}\right)^{-1} a_{5}=a_{1}+{ }_{e} a_{2}^{-1}+_{e} a_{3}+_{e} a_{2}^{-1}+_{e} a_{5},
$$

thus, by the Corollary 1.1 .18 both sides are equal, and equality in (1) holds.

(2) The idempotency of the ternary operation follows by any of Mal'cev identities.

(3) For the third statement, observe that

$$
\left[a_{1}, a_{2}, a_{3}\right]=a_{1} \Longrightarrow\left[a_{3},\left[a_{1}, a_{2}, a_{3}\right], a_{1}\right]=\left[a_{3}, a_{1}, a_{1}\right] \Longrightarrow a_{2}=a_{3},
$$

where the first equality follows from the fact that $\left[a_{3},-, a_{1}\right]$ is a function and the second equality follows by (1) and Mal'cev identities. Opposite implication is trivial.

(4) Observe that by (1), associativity and Mal'cev identities one gets,

$$
\begin{aligned}
{\left[\left[a_{1}, a_{4}, a_{5}\right],\left[a_{2}, a_{4}, a_{5}\right],\left[a_{3}, a_{4}, a_{5}\right]\right] } & =\left[\left[\left[a_{1}, a_{4}, a_{5}\right], a_{5}, a_{4}\right], a_{2},\left[a_{3}, a_{4}, a_{5}\right]\right] \\
& =\left[\left[a_{1}, a_{4},\left[a_{5}, a_{5}, a_{4}\right]\right], a_{2},\left[a_{3}, a_{4}, a_{5}\right]\right] \\
& =\left[a_{1}, a_{2},\left[a_{3}, a_{4}, a_{5}\right]\right]=\left[\left[a_{1}, a_{2}, a_{3}\right], a_{4}, a_{5}\right]
\end{aligned}
$$

therefore (4) holds.

If heap is Abelian, then (1) in Corollary 1.1 .18 implies the following associative property,

$$
\left[a_{1}, a_{2},\left[a_{3}, a_{4}, a_{5}\right]\right]=\left[a_{1},\left[a_{2}, a_{3}, a_{4}\right], a_{5}\right]=\left[\left[a_{1}, a_{2}, a_{3}\right], a_{4}, a_{5}\right]
$$

which allows one to simplify the notation by omitting brackets. In this case we write

$$
\left[a_{1}, \ldots, a_{2 n+1}\right] \text { or by imitating the sum notation }\left[a_{i}\right]_{i=1}^{2 n+1}, \quad a_{1}, \ldots, a_{2 n+1} \in H \text {, }
$$

for the result of applying the Abelian heap operation $n$-times in any possible way.

Lemma 1.1.20. In an Abelian heap $H$,

$$
\left[\left[a_{i, j}\right]_{i=1}^{2 n+1}\right]_{j=1}^{2 m+1}=\left[\left[a_{i, j}\right]_{j=1}^{2 m+1}\right]_{i=1}^{2 n+1}
$$

for all $n, m \geq 0$ and for all $a_{i, j} \in H, i=1, \ldots, 2 n+1, j=1, \ldots, 2 m+1$. 
Proof. Firstly, let us prove by induction that

$$
\left[a_{i}, b_{i}, c_{i}\right]_{i=1}^{2 n+1}=\left[\left[a_{i}\right]_{i=1}^{2 n+1},\left[b_{i}\right]_{i=1}^{2 n+1},\left[c_{i}\right]_{i=1}^{2 n+1}\right]
$$

for all $n \geq 0$. For $n=0$ 1.1.3 is trivial. If $n=1$,

$$
\begin{aligned}
{\left[\left[a_{1}, b_{1}, c_{1}\right]\left[a_{2}, b_{2}, c_{2}\right]\left[a_{3}, b_{3}, c_{3}\right]\right] } & =\left[a_{1}, b_{1}, c_{1}, a_{2}, b_{2}, c_{2}, a_{3}, b_{3}, c_{3}\right]=\left[a_{1}, a_{2}, c_{1}, b_{1}, a_{3}, c_{2}, b_{2}, b_{3}, c_{3}\right] \\
& =\left[a_{1}, a_{2}, a_{3}, b_{1}, c_{1}, b_{3}, b_{2}, c_{2}, c_{3}\right] \\
& =\left[\left[a_{1}, a_{2}, a_{3}\right],\left[b_{1}, b_{2}, b_{3}\right],\left[c_{1}, c_{2}, c_{3}\right]\right]
\end{aligned}
$$

and 1.1.3 holds for $n=1$. Let us assume the induction hypothesis that (1.1.3) holds for any $n \in \mathbb{N}$, then

$$
\begin{aligned}
{\left[a_{i}, b_{i}, c_{i}\right]_{i=1}^{2 n+3} } & =\left[\left[a_{i}, b_{i}, c_{i}\right]_{i=1}^{2 n+1},\left[a_{2 n+2}, b_{2 n+2}, c_{2 n+2}\right],\left[a_{2 n+3}, b_{2 n+3}, c_{2 n+3}\right]\right] \\
& =\left[\left[\left[a_{i}\right]_{i=1}^{2 n+1},\left[b_{i}\right]_{i=1}^{2 n+1},\left[c_{i}\right]_{i=1}^{2 n+1}\right],\left[a_{2 n+2}, b_{2 n+2}, c_{2 n+2}\right],\left[a_{2 n+3}, b_{2 n+3}, c_{2 n+3}\right]\right] \\
& =\left[\left[\left[a_{i}\right]_{i=1}^{2 n+1}, a_{2 n+2}, a_{2 n+3}\right],\left[\left[b_{i}\right]_{i=1}^{2 n+1}, b_{2 n+2}, b_{2 n+3}\right],\left[\left[c_{i}\right]_{i=1}^{2 n+1}, c_{2 n+2}, c_{2 n+3}\right]\right] \\
& =\left[\left[a_{i}\right]_{i=1}^{2 n+3},\left[b_{i}\right]_{i=1}^{2 n+3},\left[c_{i}\right]_{i=1}^{2 n+3}\right]
\end{aligned}
$$

where the third equality follows by the case for $n=1$. Therefore the induction hypothesis holds for all $n \in \mathbb{N}$. Now, let us fix an arbitrary $m \in \mathbb{N}$, then 1.1 .2 holds for $n=0$ trivially and for $n=1$ we get

$$
\left[a_{1, j}, a_{2, j}, a_{3, j}\right]_{j=1}^{2 m+1}=\left[\left[a_{1, j}\right]_{j=1}^{2 m+1},\left[a_{1, j}\right]_{j=1}^{2 m+1},\left[a_{1, j}\right]_{j=1}^{2 m+1}\right]
$$

which follows by 1.1.3). Since 1.1.2 holds for $n=0,1$ let us assume the induction hypothesis that 1.1 .2 is true for any $n \in \mathbb{N}$. Then

$$
\begin{aligned}
{\left[\left[a_{i, j}\right]_{i=1}^{2 n+3}\right]_{j=1}^{2 m+1} } & =\left[\left[a_{i, j}\right]_{i=1}^{2 n+1}, a_{2 n+2, j}, a_{2 n+3, j}\right]_{j=1}^{2 m+1} \\
& =\left[\left[\left[a_{i, j}\right]_{i=1}^{2 n+1}\right]_{j=1}^{2 m+1},\left[a_{2 n+2, j}\right]_{j=1}^{2 m+1},\left[a_{2 n+3, j}\right]_{j=1}^{2 m+1}\right] \\
& =\left[\left[\left[a_{i, j}\right]_{j=1}^{2 m+1}\right]_{i=1}^{2 n+1},\left[a_{2 n+2, j}\right]_{j=1}^{2 m+1},\left[a_{2 n+3, j}\right]_{j=1}^{2 m+1}\right] \\
& =\left[\left[a_{i, j}\right]_{j=1}^{2 m+1}\right]_{i=1}^{2 n+3}
\end{aligned}
$$

where second equality follows by (1.1.3) and third by the induction hypothesis. Therefore (1.1.2) holds for all $n, m \in \mathbb{N}$. 
Lemma 1.1.21. Let $H$ be a heap. The endomaps, defined for all $e, f \in H$,

$$
\tau_{e}^{f}: H \longrightarrow H, \quad a \longmapsto[a, e, f], \quad \tau_{f}^{e}: H \longrightarrow H, \quad a \longmapsto[a, f, e]
$$

are mutually inverse heap isomorphisms.

Proof. Let $a, b, c \in H$, then

$$
\left[\tau_{e}^{f}(a), \tau_{e}^{f}(b), \tau_{e}^{f}(c)\right]=[[a, e, f],[b, e, f],[c, e, f]]=[[a, b, c], e, f]=\tau_{e}^{f}([a, b, c])
$$

where the second equality follows by (4) of Lemma 1.1.19. Thus $\tau_{e}^{f}$ is a heap homomorphism. Now, by Mal'cev identities for all $a \in H$

$$
\tau_{e}^{f}\left(\tau_{f}^{e}(a)\right)=[[a, f, e], e, f]=[a, f,[e, e, f]]=[a, f, f]=a
$$

and the composition $\tau_{f}^{e} \circ \tau_{e}^{f}$ follows analogously. Therefore $\tau_{e}^{f}$ and $\tau_{f}^{e}$ are mutually inverse heap isomorphisms.

Lemma 1.1.22. Let $f: H_{1} \rightarrow H_{2}$ be a function between heaps. Then the following are equivalent:

(1) A function $f: H_{1} \rightarrow H_{2}$ is a heap homomorphism.

(2) For all $e_{1} \in H_{1}$ and $e_{2} \in H_{2}$ there exists a group homomorphism $\hat{f}: \mathrm{G}\left(H_{1} ; e_{1}\right) \rightarrow \mathrm{G}\left(H_{2} ; e_{2}\right)$ such that $f=\tau_{e_{2}}^{f\left(e_{1}\right)} \circ \hat{f}$.

(3) There exist $e_{1} \in H_{1}$ and $e_{2} \in H_{2}$ such that $f=\tau_{e_{2}}^{f\left(e_{1}\right)} \circ \hat{f}$, for some group homomorphism $\hat{f}: \mathrm{G}\left(H_{1} ; e_{1}\right) \rightarrow \mathrm{G}\left(H_{2} ; e_{2}\right)$.

Proof. (11) $\Longrightarrow$ (2) Let $f: H_{1} \rightarrow H_{2}$ be a heap homomorphism. For any $e_{1} \in H_{1}$ and $e_{2} \in H_{2}$ let us consider $\hat{f}:=\tau_{f\left(e_{1}\right)}^{e_{2}} \circ f$. Now, since $\hat{f}\left(e_{1}\right)=\left(\tau_{f\left(e_{1}\right)}^{e_{2}} \circ f\right)\left(e_{1}\right)=$ $\left[e_{2}, f\left(e_{1}\right), f\left(e_{1}\right)\right]=e_{2}$ and $\hat{f}$ is a heap homomorphism, Corollary 1.1 .13 implies that $\hat{f}$ is required group homomorphism.

$(2) \Longrightarrow(3)$. Follows trivially, as universal quantifier implies existential one.

(3) $\Longrightarrow$ (1). If $f=\tau_{e_{2}}^{f\left(e_{1}\right)} \circ \hat{f}$, then $f$ is a composition of two heap homomorphisms, as group homomorphism between retracts is a homomorphism between heaps. Thus $f$ is a heap homomorphism. 
Lemma 1.1.23. The set $\mathbf{A h}\left(H_{1}, H_{2}\right)$ is a heap with the point-wise operation, i.e. for all $\varphi, \varphi^{\prime}, \varphi^{\prime \prime} \in \mathbf{A} \mathbf{h}\left(H_{1}, H_{2}\right)$, the function

$$
\left[\varphi, \varphi^{\prime}, \varphi^{\prime \prime}\right]: H_{1} \longrightarrow H_{2}, \quad a \longmapsto\left[\varphi(a), \varphi^{\prime}(a), \varphi^{\prime \prime}(a)\right]
$$

is a homomorphism of heaps.

Proof. Let $\varphi, \varphi^{\prime}, \varphi^{\prime \prime} \in \mathbf{A} \mathbf{h}\left(H_{1}, H_{2}\right)$ and $a, b, c \in H_{1}$, then

$$
\begin{aligned}
{\left[\varphi, \varphi^{\prime}, \varphi^{\prime \prime}\right]([a, b, c]) } & =\left[[\varphi(a), \varphi(b), \varphi(c)],\left[\varphi^{\prime}(a), \varphi^{\prime}(b), \varphi^{\prime}(c)\right],\left[\varphi^{\prime \prime}(a), \varphi^{\prime \prime}(b), \varphi^{\prime \prime}(c)\right]\right] \\
& =\left[\left[\varphi(a), \varphi^{\prime}(a), \varphi^{\prime \prime}(a)\right],\left[\varphi(b), \varphi^{\prime}(b), \varphi^{\prime \prime}(b)\right],\left[\varphi(c), \varphi^{\prime}(c), \varphi^{\prime \prime}(c)\right]\right] \\
& =\left[\left[\varphi, \varphi^{\prime}, \varphi^{\prime \prime}\right](a),\left[\varphi, \varphi^{\prime}, \varphi^{\prime \prime}\right](b),\left[\varphi, \varphi^{\prime}, \varphi^{\prime \prime}\right](c)\right],
\end{aligned}
$$

where the second equality follows by Lemma 1.1.20. Therefore $\left[\varphi, \varphi^{\prime}, \varphi^{\prime \prime}\right]$ is a heap homomorphism, and one can easily check that $\mathbf{A h}\left(H_{1}, H_{2}\right)$ is a heap, since ternary operation is given point-wise.

Proposition 1.1.24. Let $H$ be a heap and $e, f \in H$. Then e-retract $\mathrm{G}(H ; e)$ is isomorphic to f-retract $\mathrm{G}(H ; f)$ as groups.

Proof. The isomorphism is given by the map $\tau_{e}^{f}$. Since $\tau_{e}^{f}$ is an isomorphism of heaps, it is enough to show that $\tau_{e}^{f}$ as a function is a group homomorphism of retracts. Let $g, h \in H$, then

$$
\begin{aligned}
\tau_{e}^{f}\left(g+{ }_{e} h\right) & =\tau_{e}^{f}([g, e, h])=[[g, e, h], e, f]=[[g, e, f],[e, e, f],[h, e, f]] \\
& =[[g, e, f], f,[h, e, f]]=\tau_{e}^{f}(g)+{ }_{f} \tau_{e}^{f}(h)
\end{aligned}
$$

thus $\tau_{e}^{f}$ is a group homomorphism of retracts, and therefore also an isomorphism.

Corollary 1.1.25. Let $H_{1}, H_{2}$ be heaps. Then $H_{1}, H_{2}$ are isomorphic as heaps if and only if for all $e \in H_{1}$ and $g \in H_{2}, \mathrm{G}\left(H_{1} ; e\right)$ and $\mathrm{G}\left(H_{2} ; g\right)$ are isomorphic as groups.

Proof. If $\varphi: H_{1} \rightarrow H_{2}$ is an isomorphism of heaps, then it is an isomorphism of theirs retracts $\mathrm{G}\left(H_{1}, e\right)$ and $\mathrm{G}\left(H_{2}, \varphi(e)\right)$, as for all $f, h \in H_{1}$

$$
\varphi\left(f+{ }_{e} h\right)=\varphi([f, e, h])=[\varphi(f), \varphi(e), \varphi(h)]=\varphi(f)+{ }_{\varphi(e)} \varphi(h) .
$$


Since all retracts are isomorphic, we have that groups $\mathrm{G}\left(H_{1} ; e\right)$ and $\mathrm{G}\left(H_{2} ; g\right)$ are isomorphic for all $g \in H_{2}$.

Opposite direction, if $\varphi: \mathrm{G}\left(H_{1} ; e\right) \rightarrow \mathrm{G}\left(H_{2} ; f\right)$ is an isomorphism of groups, then by Lemma 1.1.9 and Corollary 1.1.18, $\varphi$ is an isomorphism of heaps $H_{1}$ and $H_{2}$.

Remark 1.1.26. For all groups $G$ the following holds,

$$
\mathrm{G}(\mathrm{H}(G) ; e)) \cong \mathrm{G}(\mathrm{H}(G) ; f))
$$

In the Abelian case one can introduce an exponent of a heap.

Definition 1.1.27. Let $H$ be a heap. We say that heap has an exponent $N \in \mathbb{N}$ if there exists $e \in H$ such that $\mathrm{G}(H ; e)$ has exponent $N$.

Observe that the preceding definition is well-defined. Since by Proposition 1.1.24, all retracts are isomorphic, and an isomorphism of groups preserves exponents.

Lemma 1.1.28. The product of heaps $H_{1}$ and $H_{2}$ is the set $H_{1} \times H_{2}$ with operation defined component-wise, i.e.

$$
\left[\left(h_{1}, h_{2}\right),\left(h_{1}^{\prime}, h_{2}^{\prime}\right),\left(h_{1}^{\prime \prime}, h_{2}^{\prime \prime}\right)\right]:=\left(\left[h_{1}, h_{1}^{\prime}, h_{1}^{\prime \prime}\right],\left[h_{2}, h_{2}^{\prime}, h_{2}^{\prime \prime}\right]\right)
$$

for all $h_{1}, h_{1}^{\prime}, h_{1}^{\prime \prime} \in H_{1}$ and $h_{2}, h_{2}^{\prime}, h_{2}^{\prime \prime} \in H_{2}$.

Proof. Let $K$ be a heap, $\varphi_{1}: K \rightarrow H_{1}$ and $\varphi_{2}: K \rightarrow H_{2}$ be heap homomorphisms. Then we can define a map $\varphi: K \rightarrow H_{1} \times H_{2}$ given by $k \mapsto\left(\varphi_{1}(k), \varphi_{2}(k)\right)$. Since $\times$ is a product of sets $\varphi$ is a well-defined function, moreover for any $k_{1}, k_{2}, k_{3} \in K$

$$
\begin{aligned}
{\left[\varphi\left(k_{1}\right), \varphi\left(k_{2}\right), \varphi\left(k_{3}\right)\right] } & =\left[\left(\varphi_{1}\left(k_{1}\right), \varphi_{2}\left(k_{3}\right)\right),\left(\varphi_{1}\left(k_{2}\right), \varphi_{2}\left(k_{2}\right)\right),\left(\varphi_{1}\left(k_{3}\right), \varphi_{2}\left(k_{3}\right)\right)\right] \\
& =\left(\left[\varphi_{1}\left(k_{1}\right), \varphi_{1}\left(k_{2}\right), \varphi_{1}\left(k_{3}\right)\right],\left[\varphi_{2}\left(k_{1}\right), \varphi_{2}\left(k_{2}\right), \varphi_{2}\left(k_{3}\right)\right]\right) \\
& =\left(\varphi_{1}\left(\left[k_{1}, k_{2}, k_{3}\right]\right), \varphi_{2}\left(\left[k_{1}, k_{2}, k_{3}\right]\right)\right)=\varphi\left(\left[k_{1}, k_{2}, k_{3}\right]\right),
\end{aligned}
$$

hence $\varphi$ is a heap homomorphism. Observe that canonical projections $\pi_{1}: H_{1} \times H_{2} \rightarrow H_{1}$, $(e, g) \mapsto e$ and $\pi_{2}: H_{1} \times H_{2} \rightarrow H_{2},(e, g) \mapsto g$ are heap homomorphisms and that

$$
\pi_{1} \circ \varphi=\varphi_{1} \quad \text { and } \quad \pi_{2} \circ \varphi=\varphi_{2} .
$$


Thus from the universal property of a product for sets, we get that $\varphi$ is a unique function and consequently a unique heap homomorphism. Therefore $H_{1} \times H_{2}$ is the product of heaps.

Corollary 1.1.29. Let $H_{1}$ and $H_{2}$ be heaps, then for all $e \in H_{1}$ and $g \in H_{2}$,

$$
\mathrm{G}\left(H_{1} \times H_{2} ;(e, g)\right)=\mathrm{G}\left(H_{1} ; e\right) \times \mathrm{G}\left(H_{2} ; g\right) \quad \& \quad H_{1} \times H_{2}=\mathrm{H}\left(\mathrm{G}\left(H_{1} ; e\right) \times \mathrm{G}\left(H_{2} ; g\right)\right)
$$

Proof. Let us start with equality of groups and denote by $+_{(e, g)}$ group operation in $\mathrm{G}\left(H_{1} \times H_{2} ;(e, g)\right)$ and by + group operation in $\mathrm{G}\left(H_{1} ; e\right) \times \mathrm{G}\left(H_{2} ; g\right)$. Observe that underlying set in both sides is $H_{1} \times H_{2}$. Consider the identity function

$$
1_{H_{1} \times H_{2}}: H_{1} \times H_{2} \rightarrow H_{1} \times H_{2}, \quad(e, g) \mapsto(e, g) .
$$

We will show that the identity is a group homomorphism. Let $\left(e_{1}, g_{1}\right),\left(e_{2}, g_{2}\right) \in H_{1} \times H_{2}$, then

$$
\begin{aligned}
& 1_{H_{1} \times H_{2}}\left(\left(e_{1}, g_{1}\right)+(e, g)\right.\left.\left(e_{2}, g_{2}\right)\right)=\left[\left(e_{1}, g_{1}\right),(e, g),\left(e_{2}, g_{2}\right)\right]=\left(\left[e_{1}, e, e_{2}\right],\left[g_{1}, g, g_{2}\right]\right) \\
&=\left(e_{1}+{ }_{e} e_{2}, g_{1}+{ }_{g} g_{2}\right)=\left(e_{1}, g_{1}\right)+\left(e_{2}, g_{2}\right) \\
&=1_{H_{1} \times H_{2}}\left(e_{1}, g_{1}\right)+1_{H_{1} \times H_{2}}\left(e_{2}, g_{2}\right),
\end{aligned}
$$

and $1_{H_{1} \times H_{2}}$ is indeed a group homomorphism. Since identity is a bijection on $H_{1} \times H_{2}$ we get that $1_{H_{1} \times H_{2}}$ is a group isomorphism.

The second equation of the corollary follows from the first and the Corollary 0.1 .18

\subsection{Quotient of a heap}

As heaps are universal algebras one can consider their quotients. In this section we introduce a definition of a normal sub-heap of a heap and show that a quotient is given by normal sub-heaps. One can also find here an interpretation of a normal sub-heap in an associated group to the heap. 
Definition 1.2.1. A sub-heap $S$ of a heap $H$ is a subset $S \subseteq H$, which is closed under the ternary operation of $H$.

Remark 1.2.2. If $X$ is a subset of a heap $H$, then the sub-heap generated by $X$ will be denoted by $\langle X\rangle$ and it is equal to the intersection of all sub-heaps containing $X$.

Example 1.2.3. Let $X=\{x\}$ be a singleton set. Then $\langle X\rangle=X$ and a singleton subset of any heap is a sub-heap of that heap.

Example 1.2.4. The heap $2 \mathbb{Z}+1$, the set of odd integers, is a sub-heap of $H(\mathbb{Z})$.

Example 1.2.5. Let $K$ be a subgroup of a group $G$. Then $\mathrm{H}(K)$ is a sub-heap of $\mathrm{H}(G)$.

Definition 1.2.6. A sub-heap $S$ is said to be normal if there exists $e \in S$ such that for all $a \in H$ and $s \in S$ there exists $t \in S$ such that

$$
[a, e, s]=[t, e, a]
$$

Lemma 1.2.7. In the definition of a normal sub-heap the existential quantifier standing next to e can be substituted by the universal one. An equivalent statement of Definition 1.2.6 is that for all $a \in H$ and $e, s \in S$ there exists $t \in S$ such that the equality (1.2.1) holds.

Proof. Let us assume that $S$ is a normal sub-heap as in Definition 1.2.6, then for any $f \in H$

$$
\tau_{e}^{f}([a, e, s])=[[a, e, f], f,[s, e, f]]=\tau_{e}^{f}([t, e, a])=[[t, e, f], f,[a, e, f]]
$$

hence since $\tau_{e}^{f}$ is an isomorphism or more precisely a bijection and $\tau_{e}^{f}(s) \in S$ for all $s \in S$, we get that 1.2.1 holds for all $e \in S$. For the opposite direction universal quantifier, obviously, implies the existential one.

Lemma 1.2.8. Every sub-heap of an Abelian heap is normal.

Proof. Simply, as in any Abelian heap $H,[a, e, s]=[s, e, a]$ for all $a, e, s \in H$. Then by taking $t=s$ in the definition one gets that any sub-heap is normal. 
Lemma 1.2.9. Let $G$ be a group. Then a subgroup $G^{\prime}$ of a group $G$ is normal if and only if $\mathrm{H}\left(G^{\prime}\right)$ is a normal sub-heap of a heap $\mathrm{H}(G)$.

Proof. Let $e$ be a neutral element of $G^{\prime}$. Then for all $g \in G$ and $g^{\prime} \in G^{\prime}$ exists $g^{\prime \prime} \in G^{\prime}$ such that

$$
\left[g, e, g^{\prime}\right]=g e^{-1} g^{\prime}=g g^{\prime}=g^{\prime \prime} g=g^{\prime \prime} e^{-1} g=\left[g^{\prime \prime}, e, g\right]
$$

where the third equality and existence of $g^{\prime \prime}$ follows by the normality of $G^{\prime}$. Thus $\mathrm{H}\left(G^{\prime}\right)$ is a normal sub-heap of $\mathrm{H}(G)$.

In the opposite direction. Assume that $\mathrm{H}\left(G^{\prime}\right)$ is a normal sub-heap of $\mathrm{H}(G)$. Then for all $g \in G$ and $g^{\prime} \in G^{\prime}$ exists $g^{\prime \prime} \in G^{\prime}$ such that

$$
g g^{\prime}=g e^{-1} g^{\prime}=\left[g, e, g^{\prime}\right]=\left[g^{\prime \prime}, e, g\right]=g^{\prime \prime} e^{-1} g=g^{\prime \prime} g,
$$

where the third equality follows by the normality of $\mathrm{H}\left(G^{\prime}\right)$. Thus $G^{\prime}$ is a normal subgroup of a group $G$.

Lemma 1.2.10. Let $\varphi: H_{1} \longrightarrow H_{2}$ be a heap homomorphism then for all $g \in \operatorname{Im}(\varphi)$ the set

$$
\operatorname{ker}_{g}(\varphi):=\left\{a \in H_{1} \mid \varphi(a)=g\right\}
$$

is a normal sub-heap of $H_{1}$. We will call this set a $g$-kernel.

Proof. Let $a \in H_{1}$ and $e, s \in \operatorname{ker}_{g}(\varphi)$. Observe that

$$
[a, e, s]=[t, e, a] \Longleftrightarrow t=[[a, e, s], a, e]
$$

for some $t \in H$. Thus it is enough to check that $t \in \operatorname{ker}_{g}(\varphi)$,

$$
\begin{aligned}
\varphi(t) & =\varphi([[a, e, s], a, e])=[[\varphi(a), \varphi(e), \varphi(s)], \varphi(a), \varphi(e)] \\
& =[[\varphi(a), g, g], \varphi(a), g]=[\varphi(a), \varphi(a), g]=g
\end{aligned}
$$

therefore $t \in \operatorname{ker}_{g}(\varphi)$ and $\operatorname{ker}_{g}(\varphi)$ is a normal sub-heap of $H_{1}$. 
Lemma 1.2.11. Every sub-heap $S$ of a heap $H$ yields an equivalence relation $\sim_{S}$ on a heap $H$ :

$a \sim_{S} b \quad$ if and only if $\exists s \in S,[a, b, s] \in S \quad$ if and only if $\forall s \in S,[a, b, s] \in S$.

Moreover if $S$ is normal then $\sim_{S}$ is a congruence.

Proof. Let us assume that $S$ is a sub-heap. Then for all $a \in H$ and $s \in S,[a, a, s]=$ $s \in S$ implies that $a \sim_{S} a$, so the relation is reflexive. If $a \sim_{S} b$, then $[a, b, s] \in S$ and $[s,[a, b, s], s]=[[s, s, b], a, s]=[b, a, s] \in S$, thus $b \sim_{S} a$ and the relation is symmetric. If $a \sim_{S} b$ and $b \sim_{S} c$, then

$$
[[a, b, s], s,[b, c, s]]=[a,[s, s, b],[b, c, s]]=[[a, b, b], c, s]=[a, c, s] \in S,
$$

therefore $a \sim_{S} c$ and the relation is transitive. Now, let us assume that $S$ is a normal sub-heap and consider elements $x \sim_{S} y, x^{\prime} \sim_{S} y^{\prime}, x^{\prime \prime} \sim_{S} y^{\prime \prime}$, then there exist $e, s, t, z \in S$ such that

$$
x=[s, e, y], x^{\prime}=\left[t, e, y^{\prime}\right], x^{\prime \prime}=\left[z, e, y^{\prime \prime}\right]
$$

and

$$
\begin{aligned}
{\left[\left[x, x^{\prime}, x^{\prime \prime}\right],\left[y, y^{\prime}, y^{\prime \prime}\right], s\right] } & =\left[\left[\left[x, x^{\prime}, x^{\prime \prime}\right], y^{\prime \prime}, y^{\prime}\right], y, s\right]=\left[\left[\left[x, x^{\prime},\left[z, e, y^{\prime \prime}\right]\right] y^{\prime \prime}, y^{\prime}\right], y, s\right] \\
& =\left[\left[x, x^{\prime},\left[z, e, y^{\prime}\right]\right], y, s\right]=\left[\left[x, x^{\prime},\left[y^{\prime}, e, z^{\prime}\right]\right], y, s\right] \\
& =\left[\left[x,\left[t, e, y^{\prime}\right],\left[y^{\prime}, e, z^{\prime}\right]\right], y, s\right]=\left[\left[x,\left[y^{\prime}, e, t^{\prime}\right],\left[y^{\prime}, e, z^{\prime}\right]\right], y, s\right] \\
& =\left[\left[x, t^{\prime},\left[e, y^{\prime},\left[y^{\prime}, e, z^{\prime}\right]\right]\right], y, s\right]=\left[\left[x, t^{\prime}, z^{\prime}\right], y, s\right]=\left[\left[z^{\prime \prime}, t^{\prime}, x\right], y, s\right] \\
& =\left[z^{\prime \prime}, t^{\prime},[x, y, s]\right] \in S,
\end{aligned}
$$

where first equality follows by Lemma 1.1.19 (1), second is a substitution $x^{\prime \prime}=\left[z, e, y^{\prime \prime}\right]$, third follows form associativity and Mal'cev identitites, fourth follows by normality of $S$, i.e $\left[z, e, y^{\prime}\right]=\left[y^{\prime}, e, z^{\prime}\right]$ for some $z^{\prime} \in S$, fifth is the substitution, sixth is normality applied to $\left[t, e, y^{\prime}\right]$, seventh and eighth follows by Lemma 1.1.19 (1), associativity and Mal'cev identities combined, ninth is a normality property and last is an associativity. Therefore $\left[x, x^{\prime}, x^{\prime \prime}\right] \sim_{S}\left[y, y^{\prime}, y^{\prime \prime}\right]$ and $\sim_{S}$ is a congruence if $S$ is a normal sub-heap. 
Theorem 1.2.12. Let $H$ be a heap and $S$ be a normal sub-heap, then $H / S$ is a heap with operation

$$
[\bar{a}, \bar{b}, \bar{c}]=\overline{[a, b, c]}
$$

where $\bar{a}$ denotes the class of $a \in H$, and the canonical map $\pi: H \rightarrow H / S$ is a heap epimorphism. Moreover if $\pi: H \rightarrow H / \sim_{S}$ is a canonical epimorphism of heaps, for some congruence $\sim_{S}$, then there exists a normal sub-heap $S$ of $H$ such that $H / S=H / \sim_{S}$

Proof. If $S$ is a normal heap then by Lemma 1.2.11, there is the congruence $\sim_{S}$ and therefore $H / S:=H / \sim_{S}$ is a well-defined heap with a canonical heap homomorphism $\pi: H \rightarrow H / \sim_{S}$. Now, if $\pi: H \rightarrow H / \sim_{S}$ is a canonical epimorphism for some congruence $\sim_{S}$, then for all $a, b \in H$ and $e \in H / \sim_{S}$,

$$
a \sim_{S} b \Longleftrightarrow \pi(a)=\pi(b) \Longleftrightarrow a \sim_{\operatorname{ker}_{e}(\pi)} b
$$

Thus both these relations are mutually equal. Moreover, $\operatorname{since} \operatorname{ker}_{e}(\pi)$ is a normal sub-heap and $H / \operatorname{ker}_{e}(\pi)=H / \sim_{\operatorname{ker}_{e}(\pi)}$ we get that $H / \operatorname{ker}_{e}(\pi)=H / \sim_{S}$

Lemma 1.2.13. Let $S$ be a non-empty sub-heap of $(H,[-,-,-])$, and consider the sub-heap relation $\sim_{S}$. Then:

(1) For all $a, b \in H$, consider the map from Lemma 1.1.21:

$$
\tau_{a}^{b}: H \longrightarrow H, \quad z \longmapsto[z, a, b]
$$

(i) The equivalence classes of $\sim_{S}$ are related by the formula:

$$
\bar{b}=\tau_{a}^{b}(\bar{a})=\left\{[z, a, b] \mid z \sim_{S} a\right\}
$$

(ii) For all $e \in S$ and $a \in H$, set $S_{e}^{a}:=\tau_{e}^{a}(S)$. Then $\bar{a}=S_{e}^{a}$.

(2) For all $a \in H$, the equivalence class $\bar{a}$ is a sub-heap of $H$. Furthermore, if $S$ is a normal sub-heap of $H$, then so are the $\bar{a}$. 
(3) Equivalence classes of $\sim_{S}$ are mutually isomorphic as heaps.

(4) For all $a \in H$, the sub-heap equivalence relation $\sim_{S}$ coincides with the sub-heap equivalence relation $\sim_{\bar{a}}$. Consequently $H / S=H / \bar{a}$.

Proof. (1)(i) Let us assume that $z \sim_{S} a$, that is, that $[z, a, s] \in S$, for all $s \in S$. If $z^{\prime}=\tau_{a}^{b}(z)=[z, a, b]$, then $\left[z^{\prime}, b, s\right]=[z, a, s]$, by the associativity and the Mal'cev property. Hence $z^{\prime} \sim_{S} b$, that is, $\tau_{a}^{b}(\bar{a}) \subseteq \bar{b}$. On the other hand, if $z^{\prime} \in \bar{b}$, then set $z=\tau_{b}^{a}\left(z^{\prime}\right)=\left[z^{\prime}, b, a\right]$. Since $\tau_{b}^{a}$ is the inverse of $\tau_{a}^{b}, z^{\prime}=\tau_{a}^{b}(z)$. Furthermore, for all $s \in S,[z, a, s]=\left[z^{\prime}, b, s\right]$, and so $[z, a, s] \in S$, since $z^{\prime} \sim_{S} b$. This proves the second inclusion $\bar{b} \subseteq \tau_{a}^{b}(\bar{a})$, and hence the required equality.

Assertion (1)(ii) follows by $1($ i) and the fact that $\bar{e}=S$.

Statement (2) follows by (1), Lemma 1.1.21 and the observation that heap isomorphisms preserve the normality.

Statement (3) is a straightforward consequence of (1) and (2).

(4) Using (1)(ii) we can argue as follows: $b \sim_{S} c$ if, and only if, there exist $s, s^{\prime} \in S$ such that $[b, c, s]=s^{\prime}$. This is equivalent to the equality $[[b, c, s], e, a]=\left[s^{\prime}, e, a\right]$, for any $a \in H$ and $e \in S$, which, by associativity, is equivalent to $[b, c,[s, e, a]]=\left[s^{\prime}, e, a\right]$. The fact that $\bar{a}=S_{e}^{a}$ implies that $b \sim_{\bar{a}} c$.

The preceding lemma implies the following theorem of J. Certaine.

Theorem 1.2.14 (J. Certaine). The subset $S$ of a heap $H$ is closed under the ternary operation if and only if $S$ is a coset of some subgroup of some retract of $H$.

Proof. Let us assume that $S$ is a sub-heap of a heap $H$. Let $e \in H$ and $s \in S$, then $\mathrm{G}\left(\tau_{s}^{e}(S) ; e\right)$ is a subgroup of $\mathrm{G}(H ; e)$ by lemmas 1.1 .12 and 1.2 .13 . Thus $S$ is a coset.

In the opposite direction. If $S=a \mathrm{H}\left(G^{\prime}\right)$ for some subgroup $G^{\prime}$ of a group $G$ and $a \in G$, then $S$ is a sub-heap of $\mathrm{H}(G)$, since

$$
\left[a g, a g^{\prime}, a g^{\prime \prime}\right]:=a g g^{-1} a^{-1} a g^{\prime \prime}=a g g^{\prime-1} g^{\prime \prime}=a\left[g, g^{\prime}, g^{\prime \prime}\right]
$$

for all $g, g^{\prime}, g^{\prime \prime} \in G^{\prime}$. 
Corollary 1.2.15. Let $G$ be a group, then $S \subseteq \mathrm{H}(G)$ is a normal sub-heap if and only if $S$ is an equivalence class for some congruence $\sim_{S}$ on $G$.

Proof. Let us assume that $S$ is an equivalence class for some congruence $\sim_{S}$ on a group $G$. Then a canonical epimorphism of groups $\pi: G \rightarrow G / \sim_{S}$ is an epimorphism of associated heaps i.e. $\pi: \mathrm{H}(G) \rightarrow \mathrm{H}\left(G / \sim_{S}\right)$. Therefore $S=\operatorname{ker}_{S}(\pi)$ and by Lemma 1.2.10, $S$ is a normal sub-heap of $\mathrm{H}(G)$.

In the opposite direction. Let $e$ be a neutral element of a group $G$. If $S$ is a normal sub-heap of a heap $\mathrm{H}(G)$, then by Theorem $1.2 .14 S=a \mathrm{H}\left(G^{\prime}\right)$, for $a \in G$ and a subgroup $G^{\prime}$ of a group $G$. Moreover, by Lemma 1.2.13 $\tau_{a}^{e}(S)=e a^{-1} a \mathrm{H}\left(G^{\prime}\right)=\mathrm{H}\left(G^{\prime}\right)$ is normal. Hence by Lemma $1.2 .9 G^{\prime}$ is a normal subgroup of a group $G$, and $S$ is an equivalence class for some congruence on $G$.

Example 1.2.16. Let us consider a heap $H(\mathbb{Z})$ and its sub-heap $2 \mathbb{Z}+1$, since the heap is Abelian we know that the sub-heap is normal. It is easy to check that $\mathrm{H}(\mathbb{Z}) /(2 \mathbb{Z}+1) \cong \mathrm{H}\left(\mathbb{Z}_{2}\right)$ and $2 \mathbb{Z}+1$ is an equivalence class of relation given by a subgroup $2 \mathbb{Z}$.

Let $\varphi: A \rightarrow B$ be a homomorphism of heaps. Then by the Theorem C.0.9 $\operatorname{Ker}(\varphi)=\left\{\left(a, a^{\prime}\right) \in A \times A \mid \varphi(a)=\varphi\left(a^{\prime}\right)\right\}$ is a congruence on $A$. Observe that in fact $\sim_{\operatorname{Ker}(\varphi)}$ and $\sim_{k_{e r e}(\varphi)}$, for any $e \in \operatorname{Im}(\varphi)$, are equal. Indeed, for all $a, a^{\prime} \in A, a \sim_{\operatorname{Ker}(\varphi)} a^{\prime}$ if and only if $\left(a, a^{\prime}\right) \in \operatorname{Ker}(\varphi)$. Equivalently $\varphi(a)=\varphi\left(a^{\prime}\right)$, and $\left[\varphi(a), \varphi\left(a^{\prime}\right), e\right]=e$ if and only if $a \sim_{k e r_{e}(\varphi)} a^{\prime}$, for any $e \in \operatorname{Im}(\varphi)$. Therefore both relations $\sim_{\operatorname{Ker}(\varphi)}$ and $\sim_{k e r_{e}(\varphi)}$ are equal.

Lemma 1.2.17. Let $\varphi: A \longrightarrow B$ be a morphism of Abelian heaps and $S \subseteq A$ be a sub-heap. Denote by $\pi: A \longrightarrow A / S, a \longmapsto \bar{a}$, the canonical projection. The sub-heap relation $\sim_{S}$ is a sub-relation of the kernel relation $\sim_{\text {Ker }}(\varphi)$ if and only if there exists a unique morphism of Abelian heaps $\tilde{\varphi}: A / S \longrightarrow B$ rendering the following diagram

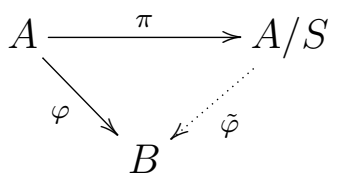


commutative. In particular, if $S \subseteq \operatorname{ker}_{e}(\varphi)$ for a certain $e \in B$, then the conclusion follows.

Proof. If $A$ is the empty heap, then there is nothing to prove. Thus, assume that $A$ is not the empty heap, which implies that $B$ is non-empty as well.

Uniqueness of $\tilde{\varphi}$ follows from the surjectivity of $\pi$. Therefore, let us check that

$$
\tilde{\varphi}: A / S \longrightarrow B, \quad \bar{a} \longmapsto \varphi(a)
$$

is a well-defined heap homomorphism. If $\bar{a}=\bar{b}$, then $a \sim_{S} b$ and so $a \sim_{\operatorname{Ker}(\varphi)} b$ as well, hence $\varphi(a)=\varphi(b)$. Thus, $\tilde{\varphi}$ is independent of the choice of the representative.

Furthermore, if there exists $e \in B$ such that $S \subseteq \operatorname{ker}_{e}(\varphi)$, then $\sim_{S}$ is a sub-relation of $\sim_{\operatorname{Ker}(\varphi)}$, since $\sim_{\operatorname{Ker}(\varphi)}=\sim_{\operatorname{ker}_{e}(\varphi)}$.

In the opposite direction. If $\varphi=\tilde{\varphi} \circ \pi$, then for any $a \sim_{S} b$

$$
\varphi(a)=\tilde{\varphi}(\pi(a))=\tilde{\varphi}(\pi(b))=\varphi(b)
$$

Thus $a \sim_{\operatorname{Ker}(\varphi)} b$ and $\sim_{S}$ is a sub-relation of the kernel relation $\sim_{\operatorname{Ker}(\varphi)}$.

In the case of $B=\operatorname{Im}(\varphi)$ and $S=\operatorname{ker}_{e}(\varphi)$, the induced map $\tilde{\varphi}$ is an isomorphism that establishes the standard first isomorphism theorem for heaps: $\operatorname{Im}(\varphi) \cong A / \operatorname{ker}_{e}(\varphi)$.

\section{$1.3 \quad$ Free heaps}

According to the title of this section, one can find in it a construction of a free heap, and a description of a connection between a free heap and a free group.

Let $X$ be a (non-empty) set. We define the set of reduced words in $X$ as the set $W(X)$ of all odd-length words in elements of $X$ such that no consecutive letters are the same, i.e.

$$
W(X):=\left\{x_{1} x_{2} \ldots x_{2 n+1} \mid x_{i} \neq x_{i+1} \in X, n \in \mathbb{N}\right\}
$$


Note that $W(X)$ is an infinite set as long as $X$ has at least two elements. Given a word $w \in W(x)$, we denote by $w^{\circ}$ the opposite word, i.e.

$$
\left(x_{1} x_{2} \ldots x_{2 n+1}\right)^{\circ}=x_{2 n+1} x_{2 n} \ldots x_{1} .
$$

On the set $W(X)$ we define a ternary operation [- - ] by grafting and pruning: given $u, v, w \in W(X)$, the reduced word $[u, v, w]$ is obtained by systematic removing (or pruning) all pairs of consecutive identical letters from the word $u v^{\circ} w$ obtained by concatenation (or grafting) of $u, v^{\circ}$ and $w$. Thus, in particular and for instance if $u$ is any reduced word and $w=x_{1} x_{2} \ldots x_{2 n+1}$, then the step-by-step pruning process leading to $[u, w, w]$ is

$$
\begin{aligned}
u w^{\circ} w & =u x_{2 n+1} x_{2 n} \ldots x_{1} x_{1} x_{2} \ldots x_{2 n+1} \longrightarrow u x_{2 n+1} x_{2 n} \ldots x_{2} x_{2} \ldots x_{2 n+1} \\
& \longrightarrow u x_{2 n+1} x_{2 n} \ldots x_{3} x_{3} \ldots x_{2 n+1} \longrightarrow \ldots \longrightarrow u x_{2 n+1} x_{2 n+1}=u .
\end{aligned}
$$

Note that this process is not affected by whether the word $u$ ends with any of the letters $x_{i}$. This shows that $[u, w, w]=u$. By similar arguments one verifies the other Mal'cev identity. Since concatenation is an associative operation and removing pairs of consecutive identical letters of several concatenated words yields the same result irrespective of the order in which concatenated words are pruned, $[---]$ is an associative operation. Thus $(W(X),[---])$ is a heap, which we denote by $\mathcal{H}(X)$.

Lemma 1.3.1. The heap $\mathcal{H}(X)$ is the free heap on $X$, i.e., for any heap $H$ and any function $\varphi: X \rightarrow H$, there exists unique filler $\widehat{\varphi}$ in the category of heaps of the following diagram:

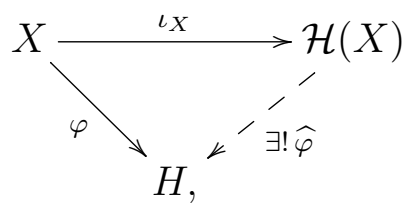

where $\iota_{X}$ is the inclusion of $X$ into $W(X)$.

Proof. Given a function $\varphi: X \rightarrow H$, the required unique heap homomorphism $\widehat{\varphi}: \mathcal{H}(X) \longrightarrow H$ is defined by

$$
\widehat{\varphi}(x):=\varphi(x), \quad \widehat{\varphi}\left(x_{1} x_{2} \ldots x_{2 n+1}\right):=\left[\widehat{\varphi}\left(x_{1} x_{2} \ldots x_{2 n-1}\right), \varphi\left(x_{2 n}\right), \varphi\left(x_{2 n+1}\right)\right] .
$$


Corollary 1.3.2. An assignment $\mathcal{H}: \operatorname{Grp} \rightarrow$ Heap, given on sets $A$ by $A \mapsto \mathcal{H}(A)$ and on functions $f: A \rightarrow B$ by $\mathcal{H}(f)=\widehat{\iota_{B} \circ f}$, is a functor. Moreover it is a left adjoint to a forgetful functor.

Proof. Proof follows analogously to the proof of Lemma 1.5.4 and Theorem 1.5.5.

For further convenience let us denote a free group generated by the set $X$ as $\mathcal{G}(X)$.

Lemma 1.3.3. Any free heap can be associated with a free group. Moreover

$$
\mathrm{H}(\mathcal{G}(X \backslash\{x\})) \cong \mathcal{H}(X)
$$

where $X$ is a non-empty set and $x \in X$.

Proof. Let $X$ be a non-empty set. The isomorphism needed to prove this statement is a unique filler of the diagram in Lemma 1.3.1, where the function $\varphi$ is defined as follows:

$$
\varphi: X \longrightarrow \mathrm{H}(\mathcal{G}(X \backslash\{x\})), \quad y \longmapsto \begin{cases}y, & y \neq x \\ e, & y=x\end{cases}
$$

where $e$ is the neutral element of $\mathcal{G}(X \backslash\{x\})$. The inverse to $\varphi$ is given by the group homomorphism (seen as a heap homomorphism) arising from the universal property of the free group $\mathcal{G}(X \backslash\{x\})$ applied to the function

$$
\psi: X \backslash\{x\} \longrightarrow \mathrm{G}(\mathcal{H}(X) ; x), \quad y \longmapsto y
$$

Corollary 1.3.4. Any non-empty sub-heap of a free heap is free.

Proof. Let us suppose that a non-empty sub-heap $S$ of the free heap $\mathcal{H}(X)$ is a non-free heap, then from Lemma $1.3 .3 \mathrm{G}(S ; e)$ is a non-free subgroup of $\mathrm{G}(\mathcal{H}(X), e) \cong \mathcal{G}(X \backslash\{e\})$ for some $e \in X$. The Nielsen-Schreier theorem [23] states that every subgroup of a free group is free, and thus we obtain a contradiction with the assumption that $\mathrm{G}(S ; e)$ is non-free, so $S$ is a free heap. 
Example 1.3.5. Let $X=\{0,1\}$ so that $\mathcal{H}(X)$ consists of all odd-length sequences of alternating digits 0 and 1 . All such sequences are symmetric, hence $w^{\circ}=w$ and the heap operation on $\mathcal{H}(X)$ is given by concatenation and pruning. By Lemma $1.3 .3 \mathcal{H}(X)$ is isomorphic with the heap associated to a free group on a singleton set (i.e. on $X$ with one element removed), so $\mathcal{H}(X)$ is the heap associated with $\mathbb{Z}$.

The idea of the construction of free Abelian heaps is to consider symmetric words of odd length in alphabet $X$,

$$
w=: x_{1} y_{1} x_{2} \ldots y_{n} x_{n+1}:, \quad x_{i}, y_{i} \in X, n \in \mathbb{N}
$$

that are defined as classes, each class of a word consists of all words created by permuting letters in the word in such a way that parity of the position of the letter in the word is preserved i.e. $x_{1} x_{2} x_{3}$ is in the same class as $x_{3} x_{2} x_{1}$ but it is not in the same class as $x_{1} x_{3} x_{2}$. Each $w$ in 1.3.1 is a set

$$
: x_{1} y_{1} x_{2} \ldots y_{n} x_{n+1}:=\left\{x_{\sigma(1)} y_{\hat{\sigma}(1)} x_{\sigma(2)} \ldots y_{\hat{\sigma}(n)} x_{\sigma(n+1)} \mid \sigma \in S_{n+1}, \hat{\sigma} \in S_{n}\right\}
$$

A symmetic word is said to be reduced if it contains only reduced words. For example, :abacd: is a symmetric reduced word, while :abcad: is not, since it contains the unreduced word $a a c b d$. The set of all symmetric reduced words of odd length on $X$ is denoted by $\bar{W}(X)$. Obviously, if $: w: \in \bar{W}(X)$, then $: w^{\circ}:=: w$. From any unreduced symmetric word one can obtain a unique symmetric reduced word by pruning. Starting with any word $x_{1} y_{1} x_{2} \ldots y_{n} x_{n+1}$ we look at all permuted words $x_{\sigma(1)} y_{\hat{\sigma}(1)} x_{\sigma(2)} \ldots y_{\hat{\sigma}(n)} x_{\sigma(n+1)}$. If any of these permuted words is not reduced, we prune it by removing pairs of consecutive identical letters. The shortest remaining word will yield the required reduced symmetric word. The heap operation on $\bar{W}(X)$ is obtained by concatenations of representatives of symmetric reduced words followed by symmetric pruning. We use notation (1.3.1) for both an unreduced word and the one to which it can be reduced. The resulting heap is the free Abelian heap on $X$ and is denoted by $\mathcal{A}(X)$.

Remark 1.3.6. One can easily employ the same isomorphism as in the proof of Lemma 1.3.3 to observe that the free Abelian heap on a non-empty set $X$ is isomorphic to the heap associated with the free Abelian group on $X \backslash\{x\}$, for any $x \in X$. 


\subsection{Coproduct of Abelian heaps}

In this section we construct a coproduct of Abelian heaps.

Given Abelian heaps $A, B$, their direct sum or coproduct $A \boxplus B$ can be constructed as follows. Start with the free Abelian heap on the disjoint union of sets $A \sqcup B, \mathcal{A}(A \sqcup B)$, and apply the ternary operations of $A$ and $B$ whenever possible to reduce words further to the point when no reduction is possible. In other words, we fix $e \in \mathcal{A}(A \sqcup B)$ and take the sub-heap $C_{e}$ of the $\mathcal{A}(A \sqcup B)$ generated by

$$
\left[\left[a, a^{\prime}, a^{\prime \prime}\right],\left[a, a^{\prime}, a^{\prime \prime}\right]_{A}, e\right], \quad\left[\left[b, b^{\prime}, b^{\prime \prime}\right],\left[b, b^{\prime}, b^{\prime \prime}\right]_{B}, e\right]
$$

where $a, a^{\prime}, a^{\prime \prime} \in A, b, b^{\prime}, b^{\prime \prime} \in B$, and $[---],[---]_{A},[---]_{B}$ are ternary operations in $\mathcal{A}(A \sqcup B), A$ and $B$, respectively, and consider the quotient heap $A \boxplus B=\mathcal{A}(A \sqcup B) / C_{e}$. One can prove that this defines a congruence on $\mathcal{A}(A \sqcup B)$ the equivalence classes of which are denoted by $\overline{: s_{1} s_{2} \ldots s_{2 n+1}}, s_{i} \in A \sqcup B$, and which form the Abelian heap $A \boxplus B$. More explicit ways of describing the elements of $A \boxplus B$ are possible.

Proposition 1.4.1. Let $A$ and $B$ be Abelian heaps.

(1) The direct sum $A \boxplus B$ contains only the following (types) of symmetric words in $A$ and $B$ :

(a) Elements $a \in A$ and $b \in B$.

(b) Three letter words $\overline{: a b b^{\prime}}$ : and $\overline{: a a^{\prime} b}$ : with $a \neq a^{\prime} \in A$ and $b \neq b^{\prime} \in B$.

(c) Alternating words $\overline{: a_{1} b_{1} a_{2} \ldots a_{n} b_{n} a_{n+1}}$ : and $\overline{: b_{1} a_{1} b_{2} \ldots b_{n} a_{n} b_{n+1}:}$, where $a_{i} \in$ $A$ and $b_{i} \in B$.

(2) Fix any $e_{A}, e_{B} \in B$. Then any of the multi-letter words in statement (1) can be written as

$$
\overline{: a b e_{B}:}, \overline{: b a e_{A}}, \overline{: a b e_{A} e_{B} \ldots e_{A} e_{B} e_{A}}: \overline{: b a e_{B} e_{A} \ldots e_{B} e_{A} e_{B}:}, \quad a \in A, b \in B
$$

Proof. (1) It is clear that $A \boxplus B$ contains words listed in (a) and (b) and that such words cannot be reduced any further. It is also clear that there could be no clusters of 
more than two consecutive letters from either $A$ and $B$. We will show that any cluster of two letters from the same alphabet can be removed from a word of length at least five. Taking into account the $A-B$ symmetry suffices it consider clusters $a b b^{\prime} a^{\prime}$ with $a, a^{\prime} \in A, b, b^{\prime} \in B$ within a symmetric word. If this word has more than five letters, then it contains an additional element of $B$. Depending on the parity of its position, it can be swapped with either $a$ or $a^{\prime}$ to form a cluster of three letters in $B$ in-between $a$ and $a^{\prime}$, which then is reduced to a single element by using the heap operation in $B$. In case the word has five letters, by swapping and using heap operations it can be reduced to an at most three letter word of type $a b b^{\prime}$ or $a a^{\prime} b$.

This completes the proof.

(2) Using the axioms of an Abelian heap and the definition of $A \boxplus B$, we can compute

$$
\overline{: a b b^{\prime}:}=\overline{\left[: a b b^{\prime}:, e_{B}, e_{B}\right]}=\overline{: a\left[b b^{\prime} e_{B}\right] e_{B}:}=\overline{: a b^{\prime \prime} e_{B}}
$$

with $b^{\prime \prime}=\left[b, b^{\prime}, e_{B}\right]$ as required. The case of $\overline{: a a^{\prime} b}$ : is dealt with in a similar way. Words in alternating letters can be transferred to the prescribed form by consecutive applying of the above procedure. Explicitly, for $w=a_{1} b_{1} \ldots a_{n-1}$,

$$
\begin{aligned}
\overline{: w b_{n-1} a_{n} b_{n} a_{n+1}}: & =\overline{\left[: w b_{n-1} a_{n} b_{n} a_{n+1}:, e_{A}, e_{A}\right]}=\overline{: w b_{n-1} a_{n} b_{n} a_{n+1} e_{A} e_{A}}: \\
& =\overline{: w b_{n-1} a_{n} e_{A} a_{n+1} b_{n} e_{A}}: \overline{: w b_{n-1} a_{n}^{\prime} b_{n} e_{A}}: \\
& =\overline{: w b_{n-1} a_{n}^{\prime} b_{n} e_{B} e_{B} e_{A}}: \overline{: w b_{n-1} e_{B} b_{n} a_{n}^{\prime} e_{B} e_{A}}=\overline{: w b_{n-1}^{\prime} a_{n}^{\prime} e_{B} e_{A}}
\end{aligned}
$$

etc., with $a_{n}^{\prime}=\left[a_{n}, e_{A}, a_{n+1}\right]$ and $b_{n-1}^{\prime}=\left[b_{n-1}, e_{B}, b_{n}\right]$.

We refer to sequences of the alternating $e_{A}$ and $e_{B}$ as to tails.

Proposition 1.4.2. Let $A$ and $B$ be Abelian heaps. Together with the inclusions $\iota_{A}: A \longrightarrow A \boxplus B, a \longmapsto a$, and $\iota_{B}: B \longrightarrow A \boxplus B, b \longmapsto b, A \boxplus B$ is a coproduct in the category of Abelian heaps.

Proof. We need to prove that given an Abelian heap $H$ and heap morphisms $f: A \longrightarrow H$ 
and $\psi: B \longrightarrow H$, there is a unique filler $\varphi \boxplus \psi$ in the diagram:

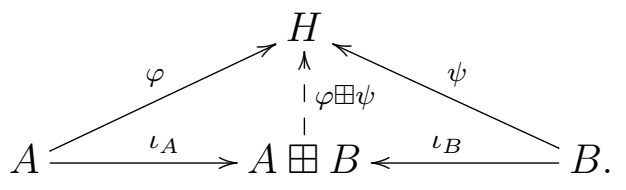

It is clear that the unique way of defining a heap homomorphism $\varphi \boxplus \psi$ that fits diagram (1.4.1) is to set $(\varphi \boxplus \psi)(a)=\varphi(a)$ and $(\varphi \boxplus \psi)(b)=\psi(b)$, for all $a \in A, b \in B$, and then extend it to words in $A \boxplus B$ letter-by-letter. We need to assure, however, that this definition is independent on the choice of representatives in the equivalence classes of symmetrised reduced words listed in, say, statement (1) of Proposition 1.4.1. Two classes can be equal if and only if they are of the same type (i.e. starting with an element of $A$ or starting with an element of $B$ as in Proposition 1.4.1(1)(c), or with two elements of $A$ or two elements of $B$ as in Proposition 1.4.1(1)(b)), as there is no way of joining elements in the same heap to produce a single element and thus reduce the length of the word or change its type. We look at these possibilities in turn.

If $\overline{: a b_{1} b_{2}:}=\overline{: a^{\prime} b_{1}^{\prime} b_{2}^{\prime}}$, then using the Mal'cev identity, symmetry and the definition of heap operation in $A \boxplus B$ we find

$$
a^{\prime}=\overline{: a^{\prime} a b_{1} b_{2} a b_{1} b_{2}:}=\overline{: a^{\prime} a^{\prime} b_{1}^{\prime} b_{2}^{\prime} a b_{1} b_{2}:}=\overline{: b_{1}^{\prime} b_{2}^{\prime} a b_{1} b_{2}:}=\overline{: a b_{2}^{\prime}\left[b_{1}^{\prime}, b_{1}, b_{2}\right]},
$$

since $A$ and $B$ are disjoint in $A \boxplus B$ and relation is given by symmetrisation and pruning this implies that $a^{\prime}=a$ and $b_{2}^{\prime}=\left[b_{1}^{\prime}, b_{1}, b_{2}\right]$. Therefore,

$$
\begin{aligned}
(\varphi \boxplus g)\left(\overline{: a^{\prime} b_{1}^{\prime} b_{2}^{\prime}:}\right) & =\left[\varphi\left(a^{\prime}\right), g\left(b_{1}^{\prime}\right), g\left(b_{2}^{\prime}\right)\right]=\left[\varphi(a), g\left(b_{1}^{\prime}\right),\left[g\left(b_{1}^{\prime}\right), g\left(b_{1}\right), g\left(b_{2}\right)\right]\right] \\
& =\left[\varphi(a), g\left(b_{1}\right), g\left(b_{2}\right)\right]=(\varphi \boxplus g)\left(\overline{: a b_{1} b_{2}}\right),
\end{aligned}
$$

where we used that $g$ is a heap morphism and the Mal'cev identity. The other case in Proposition 1.4.1 (1)(b) follows by the $A$ - $B$-symmetry.

To treat the words listed in Proposition 1.4.1 (1)(c) we first claim that if

$$
\overline{: a_{1} b_{1} a_{2} \ldots a_{n} b_{n} a_{n+1}}:=\overline{: a_{1}^{\prime} b_{1}^{\prime} a_{2}^{\prime} \ldots a_{n}^{\prime} b_{n}^{\prime} a_{n+1}^{\prime}},
$$

then

$$
a_{n+1}^{\prime}=\left[a_{1}, a_{1}^{\prime}, \ldots, a_{n}^{\prime}, a_{n+1}\right] \quad \text { and } \quad b_{n}^{\prime}=\left[b_{1}, b_{1}^{\prime}, \ldots, b_{n-1}^{\prime}, b_{n}\right] \text {. }
$$


We prove this assertion by induction on $n$. The case of $n=1$ follows by similar reasoning as in the case already studied (simply replace $a$ by $a_{1}, a^{\prime}$ by $a_{1}^{\prime}, b_{2}$ by $a_{2}$ and $b_{2}^{\prime}$ by $a_{2}^{\prime}$, and use the corresponding arguments). Assume that the statement holds for some $n$, and assume that

$$
\overline{a_{1} b_{1} a_{2} \ldots a_{n+1} b_{n+1} a_{n+2}}=\overline{: a_{1}^{\prime} b_{1}^{\prime} a_{2}^{\prime} \ldots a_{n+1}^{\prime} b_{n+1}^{\prime} a_{n+2}^{\prime}}
$$

Then, first by using the Mal'cev identities, and then by the symmetry and the definition of operation in $A \boxplus B$,

$$
\begin{aligned}
\overline{: a_{2}^{\prime} b_{2}^{\prime} a_{3}^{\prime} \ldots a_{n+1}^{\prime} b_{n+1}^{\prime} a_{n+2}^{\prime}} & =\overline{: a_{1} b_{1} a_{2} \ldots a_{n+1} b_{n+1} a_{n+2} a_{1}^{\prime} b_{1}^{\prime}:} \\
& =\overline{: a_{1} b_{1} a_{2} \ldots b_{n}\left[a_{n+1}, a_{1}^{\prime}, a_{n+2}\right] b_{n+1} b_{1}^{\prime}:} \\
& =\overline{: a_{1} b_{1} a_{2} \ldots a_{n}\left[b_{n}, b_{1}^{\prime}, b_{n+1}\right]\left[a_{n+1}, a_{1}^{\prime}, a_{n+2}\right]:}
\end{aligned}
$$

As the length of the word is $2 n+1$, the inductive assumption can be applied, so that

$$
a_{n+2}^{\prime}=\left[a_{1}, a_{2}^{\prime}, a_{2}, \ldots a_{n+1}^{\prime},\left[a_{n+1}, a_{1}^{\prime}, a_{n+2}\right]\right]=\left[a_{1}, a_{1}^{\prime}, \ldots, a_{n+1}^{\prime}, a_{n+2}\right],
$$

where the fact that $A$ is an Abelian herd has been used. The formula for $b_{n+1}^{\prime}$ can be derived using the second part of the conjunction in the inductive assumption. This proves that 1.4.3 holds for all $n \in \mathbb{N}$.

In the situation (1.4.2), using (1.4.3), that both $f$ and $g$ are heap morphisms, Mal'cev 
identities and the Abelian nature of $A$ and $B$, one can compute

$$
\begin{aligned}
(\varphi \boxplus \psi) & \left(\overline{: a_{1}^{\prime} b_{1}^{\prime} \ldots b_{n}^{\prime} a_{n+1}^{\prime}}\right)= \\
& =\left[\varphi\left(a_{1}^{\prime}\right), \psi\left(b_{1}^{\prime}\right), \ldots, \varphi\left(a_{n}^{\prime}\right), \psi\left(\left[b_{1}, b_{1}^{\prime}, \ldots, b_{n-1}^{\prime}, b_{n}\right]\right), \varphi\left(a_{n+1}^{\prime}\right)\right] \\
& =\left[\varphi\left(a_{1}^{\prime}\right), \psi\left(b_{1}^{\prime}\right), \ldots, \varphi\left(a_{n}^{\prime}\right), \psi\left(b_{1}\right), \psi\left(b_{1}^{\prime}\right), \ldots, \psi\left(b_{n-1}^{\prime}\right), \psi\left(b_{n}\right), \varphi\left(a_{n+1}^{\prime}\right)\right] \\
& =\left[\varphi\left(a_{1}^{\prime}\right), \psi\left(b_{1}^{\prime}\right), \psi\left(b_{1}^{\prime}\right), \varphi\left(a_{2}^{\prime}\right) \ldots, \psi\left(b_{1}\right), \varphi\left(a_{n}^{\prime}\right), \ldots, \psi\left(b_{n-1}^{\prime}\right), \psi\left(b_{n}\right), \varphi\left(a_{n+1}^{\prime}\right)\right] \\
& =\left[\varphi\left(a_{1}^{\prime}\right), \varphi\left(a_{2}^{\prime}\right), \ldots, \psi\left(b_{1}\right), \varphi\left(a_{n}^{\prime}\right), \ldots, \psi\left(b_{n-1}^{\prime}\right), \psi\left(b_{n}\right), \varphi\left(a_{n+1}^{\prime}\right)\right] \\
& =\ldots=\left[\varphi\left(a_{1}^{\prime}\right), \psi\left(b_{1}\right), \varphi\left(a_{2}^{\prime}\right), \ldots, \varphi\left(a_{n}^{\prime}\right), \psi\left(b_{n}\right), \varphi\left(\left[a_{1}, a_{1}^{\prime}, \ldots, a_{n}^{\prime}, a_{n+1}\right]\right)\right] \\
& =\left[\varphi\left(a_{1}^{\prime}\right), \psi\left(b_{1}\right), \varphi\left(a_{2}^{\prime}\right), \ldots, \varphi\left(a_{n}^{\prime}\right), \psi\left(b_{n}\right), \varphi\left(a_{1}\right), \varphi\left(a_{1}^{\prime}\right), \ldots, \varphi\left(a_{n}^{\prime}\right), \varphi\left(a_{n+1}\right)\right] \\
& =\left[\varphi\left(a_{1}^{\prime}\right), \varphi\left(a_{1}^{\prime}\right), \psi\left(b_{1}\right), \varphi\left(a_{2}^{\prime}\right), \ldots, \varphi\left(a_{n}^{\prime}\right), \varphi\left(a_{1}\right), \psi\left(b_{n}\right), \ldots, \varphi\left(a_{n}^{\prime}\right), \varphi\left(a_{n+1}\right)\right] \\
& =\left[\psi\left(b_{1}\right), \varphi\left(a_{2}^{\prime}\right), \ldots, \varphi\left(a_{n}^{\prime}\right), \varphi\left(a_{1}\right), \psi\left(b_{n}\right), \ldots, \varphi\left(a_{n}^{\prime}\right), \varphi\left(a_{n+1}\right)\right] \\
& =\ldots=\left[\varphi\left(a_{1}\right), \psi\left(b_{1}\right), \ldots, \varphi\left(a_{n}\right), \psi\left(b_{n}\right), \varphi\left(a_{n+1}\right)\right] \\
& =(\varphi \boxplus \psi)\left(\overline{: a_{1} b_{1} \ldots b_{n} a_{n+1}}\right)
\end{aligned}
$$

Thus the definition of $\varphi \boxplus \psi$ is independent on the choice of the representatives in this case. The case of the alternating words starting with elements in $B$ is dealt with in a symmetric manner (or follows by the $A-B$ symmetry). This completes the proof of the proposition.

Remark 1.4.3. Note that although Abelian heaps $A$ and $B$ can be made into Abelian groups by fixing neutral elements, say $e_{A} \in A$ and $e_{B} \in B$, the direct sum of Abelian heaps $A \boxplus B$ is not the same as the heap associated to the direct sum of the corresponding groups, i.e. $A \boxplus B \neq \mathrm{H}\left(\mathrm{G}\left(A ; e_{A}\right) \oplus \mathrm{G}\left(B ; e_{B}\right)\right)$. Since $\varphi$ and $\psi$ are heap morphisms in the diagram (1.4.1), there is no need for $e_{A}$ and $e_{B}$ to be mapped to the same element of $H$ that could serve for the neutral element of the induced group structure.

As in the case of Abelian groups, the explicit description of the direct sum of two Abelian heaps in Proposition 1.4.1 can be extended to families of Abelian heaps. In case of the family $\left(A_{x}\right)_{x \in X}$, the direct sum $\underset{x \in X}{\boxplus} A_{x}$, in addition to single and three letter

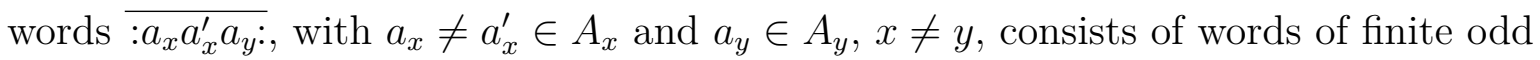
length in which neighbouring letters come from different heaps, and in which letters 
from the same heap, say $A_{x}$, are separated by odd number of letters from heaps not labelled by $x$.

The following proposition provides one with a group-theoretic description of the coproduct of Abelian heaps.

Proposition 1.4.4. Let $A$ and $B$ be Abelian heaps, then

$$
A \boxplus B \cong \mathrm{H}\left(\mathrm{G}\left(A ; e_{A}\right) \oplus \mathrm{G}\left(B ; e_{B}\right) \oplus \mathbb{Z}\right) .
$$

Proof. The functions

$$
\begin{aligned}
& \varphi_{A}: A \longrightarrow \mathrm{H}\left(\mathrm{G}\left(A ; e_{A}\right) \oplus \mathrm{G}\left(B ; e_{B}\right) \oplus \mathbb{Z}\right), a \longmapsto\left(a, e_{B}, 0\right)=a, \\
& \varphi_{B}: B \longrightarrow \mathrm{H}\left(\mathrm{G}\left(A ; e_{A}\right) \oplus \mathrm{G}\left(B ; e_{B}\right) \oplus \mathbb{Z}\right), \quad b \longmapsto\left(e_{A}, b, 1\right)=b+1,
\end{aligned}
$$

with understanding that whenever terms are written additively in the codomain $e_{A}=$ $e_{B}=0$, are heap homomorphisms. By the universal property of coproducts (cf. the diagram in proof of Proposition 1.4.2 there exists a unique homomorphism

$$
\varphi: A \boxplus B \longrightarrow \mathrm{H}\left(\mathrm{G}\left(A ; e_{A}\right) \oplus \mathrm{G}\left(B ; e_{B}\right) \oplus \mathbb{Z}\right),
$$

which restricts to $\varphi_{A}$ on $A$ and $\varphi_{B}$ on $B$. In terms of words in Proposition 1.4.1 (2) the homomorphism $\varphi$ comes out as

$$
\begin{aligned}
& \varphi\left(\overline{: a b e_{B}}\right)=a-b, \quad \varphi \underbrace{\left(\overline{: a b e_{A} e_{B} \ldots e_{A} e_{B} e_{A}}\right)}_{e_{A} \text { appears } n \text {-times }}=a-b-n, \\
& \varphi\left(\overline{: b a e_{A}:}\right)=b-a+1, \quad \underbrace{\left(\overline{: b a e_{B} e_{A} \ldots e_{B} e_{A} e_{B}}\right)}_{e_{B} \text { appears } n \text {-times }}=b-a+n+1 .
\end{aligned}
$$

The inverse of $\varphi$ is the filler of the coproduct diagram in the category of groups and is determined by

$$
\begin{gathered}
\varphi^{-1}: \mathrm{G}\left(A ; e_{A}\right) \oplus \mathrm{G}\left(B ; e_{B}\right) \oplus \mathbb{Z} \longrightarrow \mathrm{G}\left(A \boxplus B ; e_{A}\right), \\
0 \longmapsto e_{A}, \quad 1 \longmapsto e_{B}, \quad a \longmapsto a, \quad b \longmapsto \overline{: b e_{B} e_{A}},
\end{gathered}
$$

for all $a \in A$ and $b \in B$. Therefore, since any homomorphism of groups is a homomorphism of heaps, we conclude that $\varphi^{-1}$ is a homomorphism of heaps. Clearly, compositions of $\varphi$ and $\varphi^{-1}$ give identities so $\varphi$ is an isomorphism of heaps as required. 
Observe that even the coproduct of Abelian heaps is no longer a sub-heap of the product of heaps in contrast to what happens in the categories of groups.

Since the coproduct is an associative operation on a category, the identification of Proposition 1.4.4 can be iterated and transferred easily to coproducts of any finite (or infinite) number of heaps. In particular, we obtain

Remark 1.4.5. Even though we know that a coproduct of non-Abelian heaps exists and intuitively we can expect what it is, the detailed construction of it is not that easy as one need to deal with much more complicated associative law.

Corollary 1.4.6. Let $X=\left\{x_{1} \ldots x_{n}\right\}$ be a finite set. Then

$$
\mathcal{H}\left(\left\{x_{1}\right\}\right) \boxplus \mathcal{H}\left(\left\{x_{2}\right\}\right) \boxplus \ldots \boxplus \mathcal{H}\left(\left\{x_{n}\right\}\right) \cong \mathrm{H}\left(\mathbb{Z}^{n-1}\right) \cong \mathcal{A}(X) .
$$

Proof. The free heap on a singleton set is the singleton set itself, and thus the associated (Abelian) group is the trivial group 0. The first isomorphism thus follows from Proposition 1.4.4. The second isomorphism follows by Remark 1.3.6.

Example 1.4.7. Let us take heaps $A=\left\{0_{A}, 1_{A}\right\}$ and $B=\left\{0_{B}, 1_{B}\right\}$ each associated with the group $C_{2}$, and choose $0_{A}$ and $0_{B}$ as distinguished elements of statement (2) in Proposition 1.4.1. Proposition 1.4.4 implies that $A \boxplus B \cong H\left(C_{2} \oplus C_{2} \oplus \mathbb{Z}\right)$. Moreover, by choosing $G\left(A \boxplus B ; 0_{A}\right)$ and looking at the elements from Proposition 1.4.1 we can deduce that tails of the form $0_{B} 0_{A} \ldots 0_{A} 0_{B}$ and $0_{A} 0_{B} \ldots 0_{B} 0_{A}$ represent numbers of $\mathbb{Z}$ in the direct sum.

\subsection{Construction of a free group by heaps}

The main goal of this section is to construct a left adjoint functor to the functor $\mathrm{H}: \mathrm{Grp} \rightarrow$ Heap.

Let $\{*\}$ be a singleton heap. For any heap $H$, one can consider the group $\mathrm{Gr}_{*}(H):=$ $\mathrm{G}(H \boxplus\{*\} ; *)$. The following lemma shows that this group has a very interesting universal property, which will be essential in the construction of the adjoint. 
Lemma 1.5.1. Let $H$ be a heap, $S$ be a group and $f: H \rightarrow \mathrm{H}(S)$ be a heap homomorphism. Then there exists a unique group homomorphism $\operatorname{Gr}_{*}(f): \operatorname{Gr}_{*}(H) \rightarrow S$ such that $f=\mathrm{H}\left(\mathrm{Gr}_{*}(f)\right) \circ \iota_{H}$, where $\iota_{H}$ is a canonical injection into coproduct. In other words, diagram

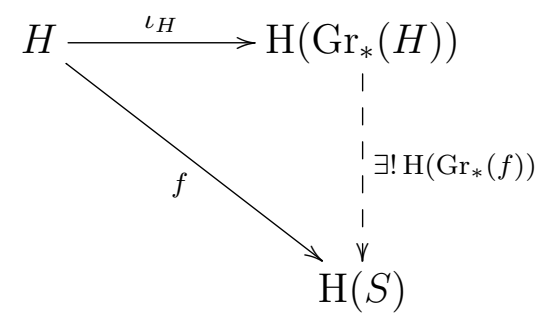

commutes, where $\exists ! \mathrm{H}\left(\mathrm{Gr}_{*}(f)\right)$ reads "there exists exactly one homomorphism of groups $\operatorname{Gr}_{*}(f)$ ". The pair $\left(\operatorname{Gr}_{*}(H), \iota_{H}\right)$ is a universal arrow, see [24, Section III.1].

Proof. Observe that by the universal property of coproduct for all groups $S$ and homomorphisms of heaps $f: H \rightarrow \mathrm{H}(S), g:\{*\} \rightarrow \mathrm{H}(S)$ diagram

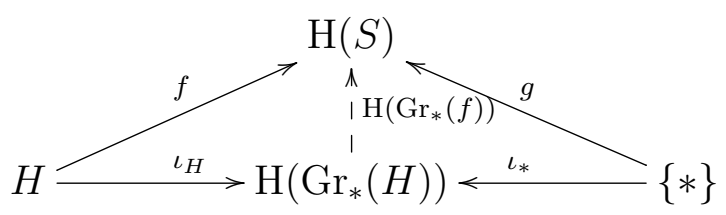

commutes. Every homomorphism of groups is a homomorphism of associated heaps. Moreover, a homomorphism of heaps is a homomorphism of retracts if, and only if it maps a neutral element to a neutral element. Hence, $\mathrm{H}\left(\mathrm{Gr}_{*}(f)\right)$ is a homomorphism of retracts if and only if $g\left(\iota_{*}(*)\right)$ is a neutral element of $S$. Observe that $g$ is unique, since $\{*\}$ is a singleton heap. Therefore $\mathrm{H}\left(\mathrm{Gr}_{*}(f)\right)$ is a unique homomorphism of heaps such that it is also a homomorphism of groups to which heaps were associated. Thus, the preceding diagram commutes.

Another important observation is that a canonical injection $\iota_{H}$ has some sort of cancellation property.

Lemma 1.5.2. Let $H, L$ be heaps and $f, g: \mathrm{H}\left(\mathrm{Gr}_{*}(H)\right) \rightarrow L$ be homomorphisms of heaps such that $f\left(\iota_{*}(*)\right)=g\left(\iota_{*}(*)\right)$, then $f \circ \iota_{H}=g \circ \iota_{H}$ implies $f=g$. 
Proof. Let us consider a homomorphism of heaps $f: \mathrm{H}\left(\mathrm{Gr}_{*}(H)\right) \rightarrow L$. One can easily observe that by the uniqueness of a coproduct map $f=\left(f \circ \iota_{H}\right) \boxplus\left(f \circ \iota_{*}\right)$. Thus, because $f\left(\iota_{*}(*)\right)=g\left(\iota_{*}(*)\right)$ and $f \circ \iota_{H}=g \circ \iota_{H}$, we get that

$$
f=\left(f \circ \iota_{H}\right) \boxplus\left(f \circ \iota_{*}\right)=\left(g \circ \iota_{H}\right) \boxplus\left(g \circ \iota_{*}\right)=g .
$$

Therefore, $f\left(\iota_{*}(*)\right)=g\left(\iota_{*}(*)\right)$ and $\left(f \circ \iota_{H}\right)=\left(g \circ \iota_{H}\right)$ implies $f=g$.

Corollary 1.5.3. Let $e \in L$. If $f, g: \mathrm{Gr}_{*}(H) \rightarrow \mathrm{G}\left(L,+_{e}\right)$, are homomorphisms of groups, then $f \circ \iota_{H}=g \circ \iota_{H}$ implies $f=g$.

Proof. This follows by Lemma 1.5.2 since a homomorphism of heaps $\mathrm{H}(f)$ is equal to a homomorphism of groups $f$ as functions.

Now, we are ready to describe the functor. Let us consider an assignment Gr : Heap $\rightarrow$ Grp given on a heap $H$ by $H \mapsto \operatorname{Gr}_{*}(H)$. One can easily see that it is a well-defined function. The assignment is given for all homomorphisms of heaps $f: H \rightarrow H^{\prime}$ by $f \mapsto \operatorname{Gr}_{*}\left(\iota_{H^{\prime}} \circ f\right)$ and is well-defined, since $\iota_{H}^{\prime} \circ f$ is a composition of homomorphisms of heaps, so it is a homomorphism of heaps. Therefore by the universal property of $\mathrm{Gr}_{*}, \mathrm{Gr}_{*}\left(\iota_{H^{\prime}} \circ f\right)$ is a homomorphism of groups.

Lemma 1.5.4. The assignment $\mathrm{Gr}:$ Heap $\rightarrow \operatorname{Grp}$ is a functor.

Proof. In the previous discussion, we explained that both assignments are well-defined functions. Thus, we have to show that functor preserves identity and composition.

Obviously $\operatorname{Gr}_{*}\left(\iota_{H} \circ 1_{H}\right)=1_{\mathrm{Gr}_{*}(H)}$.

For the composition let us assume that $f: H \rightarrow H^{\prime}$ is a homomorphism of heaps, then $\iota_{H^{\prime}} \circ f$ is a composition of homomorphisms of heaps, hence $\iota_{H^{\prime}} \circ f: H \rightarrow \mathrm{H}\left(\mathrm{Gr}\left(H^{\prime}\right)\right)$ ) is a homomorphism of heaps. If $f: H \rightarrow H^{\prime}$ and $g: H^{\prime} \rightarrow H^{\prime \prime}$ are homomorphisms of heaps, then

$$
\begin{aligned}
\operatorname{Gr}(g \circ f) \circ \iota_{H} & =\operatorname{Gr}_{*}\left(\iota_{H^{\prime \prime}} \circ g \circ f\right) \circ \iota_{H}=\iota_{H^{\prime \prime}} \circ g \circ f=\operatorname{Gr}(g) \circ \iota_{H^{\prime}} \circ f \\
& =\operatorname{Gr}(g) \circ \operatorname{Gr}(f) \circ \iota_{H},
\end{aligned}
$$


where all the equalities follow by Lemma 1.5.1 applied multiple times. Now, since $\operatorname{Gr}(g \circ f) \circ \iota_{H}=\operatorname{Gr}(g) \circ \operatorname{Gr}(f) \circ \iota_{H}$ and $\operatorname{Gr}(g \circ f), \operatorname{Gr}(g), \operatorname{Gr}(f)$ are homomorphisms of groups, applying Corollary 1.5.3, one gets that $\operatorname{Gr}(g \circ f)=\operatorname{Gr}(g) \circ \operatorname{Gr}(f)$. Therefore an assignment $\mathrm{Gr}$ preserves composition, hence $\mathrm{Gr}$ is a functor.

The following theorem confirms that $\mathrm{Gr}$ is a desirable functor.

Theorem 1.5.5. The functor $\mathrm{Gr}$ is a left adjoint to the functor $\mathrm{H}$.

Proof. For all heaps $H$ and groups $G$ let us consider functions between sets of morphisms:

$$
\begin{gathered}
\varphi_{H, G}: \operatorname{Grp}(\operatorname{Gr}(H), G) \longrightarrow \operatorname{Heap}(H, \mathrm{H}(G)), \quad f \longmapsto \mathrm{H}(f) \circ \iota_{H}, \\
\varphi_{H, G}^{-1}: \operatorname{Heap}(H, \mathrm{H}(G)) \longrightarrow \operatorname{Grp}(\operatorname{Gr}(H), G), \quad f \mapsto \mathrm{Gr}_{*}(f) .
\end{gathered}
$$

To show that $\varphi_{H, G}$ is a bijection let $f \in \operatorname{Heap}(H, \mathrm{H}(G))$ and $g \in \operatorname{Grp}(\operatorname{Gr}(H), G)$, then

$$
\varphi_{H, G} \circ \varphi_{H, G}^{-1}(f)=\varphi_{H, G}\left(\operatorname{Gr}_{*}(f)\right)=\mathrm{H}\left(\operatorname{Gr}_{*}(f)\right) \circ \iota_{H}=f,
$$

where the last equality follows by Lemma 1.5.1, and

$$
\varphi_{H, G}^{-1} \circ \varphi_{H, G}(g)=\mathrm{Gr}_{*}\left(\mathrm{H}(g) \circ \iota_{H}\right)=g
$$

where the last equality follows by the uniqueness of the morphism $\mathrm{Gr}_{*}\left(\mathrm{H}(f) \circ \iota_{H}\right)$. Hence, $\varphi_{H, G}^{-1}$ is an inverse to $\varphi_{H, G}$. Thus, $\varphi_{H, G}$ is a bijection.

To check naturality conditions, let $G, S$ be groups, $H, L$ be heaps and consider homomorphisms $f: \operatorname{Gr}(H) \longrightarrow G, \alpha: L \longrightarrow H$ and $g: G \longrightarrow S$. Then

$$
\varphi_{L, G}(f \circ \operatorname{Gr}(\alpha))=\mathrm{H}(f \circ \operatorname{Gr}(\alpha)) \circ \iota_{L}=\mathrm{H}(f) \circ \mathrm{H}(\operatorname{Gr}(\alpha)) \circ \iota_{L}=\mathrm{H}(f) \circ \iota_{H} \circ \alpha=\varphi_{H, G}(f) \circ \alpha,
$$

by applying Lemma 1.5 .1 multiple times. Similarly,

$$
\varphi_{H, S}(g \circ f)=\mathrm{H}(g \circ f) \circ \iota_{H}=\mathrm{H}(g) \circ \varphi_{H, G}(f) .
$$

Therefore $\varphi$ is a natural isomorphism and the functor Gr is a left adjoint to the functor $\mathrm{H}$. 
To underline the meaning of the preceding theorem in the context of groups let us consider the following diagram

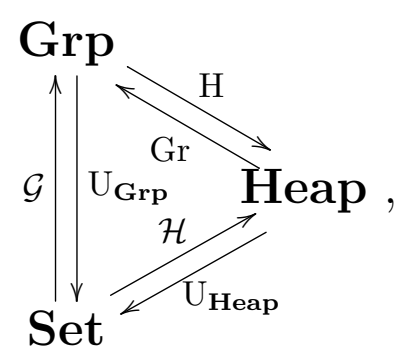

where $\mathrm{U}_{\mathbf{G r p}}$ is a forgetful functor and $\mathcal{G}$ is its left adjoint, the free functor.

The first observation is that all the opposite arrows are adjoints to each other.

The second observation is that a composition of functors,

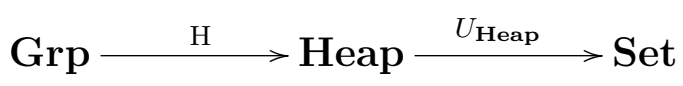

is the forgetful functor $\mathrm{U}_{\mathbf{G r p}}$ since for any group $G, \mathrm{H}(G)$ and $G$ are equal sets, and every homomorphism of groups $f$ is the same function as $\mathrm{H}(f)$. These two observations leads to the following corollaries.

Corollary 1.5.6. The functor $\mathrm{Gr} \circ \mathcal{H}:$ Set $\rightarrow \operatorname{Grp}$ is a free functor, i.e. it is a left adjoint to the functor $\mathrm{U}_{\mathbf{G r p}}: \mathbf{G r p} \rightarrow$ Set.

Proof. Observe that for any group $G$ and set $S$,

$\operatorname{Grp}(\operatorname{Gr}(\mathcal{H}(S)), G) \cong \operatorname{Heap}(\mathcal{H}(S), \mathrm{H}(G)) \cong \operatorname{Set}\left(S, \mathrm{U}_{\text {Heap }}(\mathrm{H}(G))\right)=\operatorname{Set}\left(S, \mathrm{U}_{\mathbf{G r p}}(G)\right)$

Thus $\operatorname{Gr} \circ \mathcal{H} \dashv \mathrm{U}_{\mathbf{G r p}}$.

Corollary 1.5.7. For any set $X,(\operatorname{Gr} \circ \mathcal{H})(X) \cong \mathcal{G}(X)$.

Proof. Since both functors $\operatorname{Gr} \circ \mathcal{H}$ and $\mathcal{G}$ are left adjoints to the forgetful functor, they are naturally isomorphic, see [24, Corollary 1, page 85].

To summarise, we have shown that a free functor from the category of sets to the category of groups is decomposable into two functors, through the category of heaps. The description of the free functor provides a method to construct a free group. 
Sadly, in general, it is not an easy task to describe a coproduct of heaps. One intuitively knows it is a quotient of a free heap over the disjoint union. The choice of generators for the normal sub-heap is at least tricky, because one must at the same time deal with the allocations of a ternary operation in elements of the free heap, see the associativity rule Lemma 1.1.19.1).

Fortunately, since we are interested in a composition of functors $\mathrm{Gr} \circ \mathcal{H}$, we only need to consider the coproduct of two heaps, the singleton heap and a free heap $\mathcal{H}(X)$, for any set $X$. Observe that a heap described on the singleton set is unique up to isomorphism. Thus, we can identify a singleton heap with a free heap $\mathcal{H}(\{*\})$. Now, by definition of $\operatorname{Gr} \circ \mathcal{H},(\operatorname{Gr} \circ \mathcal{H})(X)=\mathrm{G}(\mathcal{H}(X) \boxplus \mathcal{H}(\{*\}) ; *)$, but $\mathcal{H}$ is a left adjoint functor to the forgetful functor, so it preserves coproducts. Therefore, we have that $\mathcal{H}(X) \boxplus \mathcal{H}(\{*\}) \cong \mathcal{H}(X \sqcup\{*\})$. Hence, we start with taking a set $X$, then consider a disjoint union with $\{*\}$, construct a free heap over $X \sqcup\{*\}$ and take a retract of that heap in $*$. The obtained retract is a free group. Even though one can argue that we still add a disjoint element $*$, in this setup, it has a proper algebraic interpretation in the category of heaps, i.e. taking a coproduct of a free heap with a singleton heap. In contrast to Construction B.1.5, we do not add artificially the set $X^{-1}$ to get inverses, as inverses in the retract are words of the form $[*, w, *]$, for any $w \in \mathcal{H}(X \sqcup\{*\})$. 


\section{Part II}

\section{Trusses}





\section{Chapter 2}

\section{In the world of trusses}

In this chapter we generalise the definition of a truss from [15] by forgetting distributive laws. The generalisation is called a pre-truss. Next, we identify special cases of pretrusses with near-rings and skew braces. In Section 2.2, we describe the congruences of pre-trusses in terms of algebraic structure of paragons, which in the case of rings are congruence classes. The last section is mainly an investigation when units of a ring form a congruence class. All the sections of this chapter can be found in Section 3 of [26]. All the basic definitions and facts about near-rings and skew braces necessary to understand this chapter can be found in Appendix B.

\subsection{Pre-trusses, near-trusses and trusses}

The aim of this section is to introduce heaps with an additional semigroup operation and describe their relations with well-known algebraic structures.

\section{Definition 2.1.1.}

(1) A pre-truss is a heap $(T,[-,-,-])$ together with an associative binary operation called a multiplication (denoted by a juxtaposition or $\cdot$ ).

(2) A pre-truss $T$ satisfying the left distributive law:

$$
a[b, c, d]=[a b, a c, a d], \quad \text { for all } a, b, c, d \in T,
$$

is called a near-truss. 
(3) A near-truss $T$ satisfying the right distributive law

$$
[b, c, d] a=[b a, c a, d a], \quad \text { for all } a, b, c, d \in T
$$

is called a skew truss.

(4) A skew truss such that the underlying heap is Abelian is called a truss.

Every one of the above notions is said to be unital provided the binary operation has an identity (denoted by 1) and commutative if for all $a, b \in T, a b=b a$.

Definition 2.1.2. A homomorphism of (pre-, near-, skew) trusses is a homomorphism of heaps that is also a homomorphism of semigroups (or monoids in the unital case).

It is clear from this definition that the image of a homomorphism of (pre-, near-, skew) trusses is itself a (pre-, near-, skew) truss.

Definition 2.1.3. A left (resp. right) absorber is an element $a$ of a pre-truss $T$ such that, for all $t \in T, t a=a$ (resp. at $=a$ ). We say that $a$ is an absorber if it is a left and right absorber.

Lemma 2.1.4. It is worth noting that if a pre-truss $T$ has both a left and a right absorber, then they necessarily coincide, in particular an absorber is unique.

Proof. Let us assume that $l \in T$ is a left absorber and $r \in T$ is a right absorber. Then

$$
l=r \cdot l=r
$$

so $l$ is a unique two-sided absorber.

Lemma 2.1.5. Furthermore, since homomorphisms of pre-trusses preserve multiplication, if $f: T \longrightarrow T^{\prime}$ is a morphism and $e$ is a left (resp. right) absorber in $T$, then $f(e)$ is a left (resp. right) absorber in the pre-truss $f(T)$.

Proof. Let $l \in T$ ba a left absorber, then for all $t \in T$

$$
f(t) \cdot f(l)=f(t \cdot l)=f(l),
$$

and therefore $f(l)$ is a left absorber in $f(T)$. 
Now let us introduce a set of lemmas which will provide a plenty of examples of pre-trusses.

Lemma 2.1.6. Let $T$ be a truss. Then $e \in T$ is an absorber if and only if $R=$ $(\mathrm{G}(T ; e), \cdot)$ is a ring. We call such $T$ a truss associated with a ring $R$ or a ring-type truss, and denote it by $\mathrm{T}(R)$.

Proof. If $R$ is a ring, then obviously $e$ is an absorber.

In the opposite direction. If $e$ is an absorber, then it is enough to check the distributivity as $\mathrm{G}(T ; e)$ is a group and - is associative. For all $a, b, c \in T$,

$$
\begin{aligned}
& a(b+e c)=a[b, e, c]=[a b, a e, a c]=[a b, e, a c]=a b+e a c, \\
& \left(b++_{e} c\right) a=[b, e, c] a=[b a, e a, c a]=[b a, e, c a]=b a+{ }_{e} c a,
\end{aligned}
$$

so distributivity holds and therefore $R=(\mathrm{G}(T ; e), \cdot)$ is a ring.

Remark 2.1.7. Observe that an absorber is unique, therefore assignment of a ring to a truss through retract is unique.

Corollary 2.1.8. A ring $R$ is unital if and only if $\mathrm{T}(R)$ is unital.

Lemma 2.1.9. Let $T$ be a near-truss. Then $e \in T$ is a left absorber if and only if $N=(\mathrm{G}(T ; e), \cdot)$ is a near-ring. We call such $T$ a truss associated with a near-ring $N$ or a ring-type near-truss and denote it by $\mathrm{T}(N)$.

Proof. Proof is almost the same as proof of Lemma 2.1.6, the only difference is that we need to show only left distributivity.

Remark 2.1.10. Observe that in a near-truss there can be more than one left absorber, therefore the assignment of a near-ring to a truss is not necessarily unique.

Another class of examples of near-trusses is given by skew braces, see Definition B.2.26. The following lemma gives a full characterisation of near-trusses that can be associated with a skew brace by taking a retract. 
Lemma 2.1.11. Let $T$ be a unital near-truss. Then $B=(\mathrm{G}(T ; 1), \cdot)$ is a skew brace if and only if $(T, \cdot)$ is a group with neutral element 1 . We call such $T$ a brace-type near-truss and denote it by $\mathrm{T}(B)$.

Proof. If $B$ is a skew brace, then it is obvious that $(T, \cdot)$ is a group with neutral element 1.

Now, let us assume that $(T, \cdot)$ is a group, it is enough to show that the left distributive law holds. Indeed, as for all $a, b, c \in T$,

$$
\begin{aligned}
a\left(b+{ }_{1} c\right) & =a[b, 1, c]=[a b, a, a c]=[[a b, 1,1], a,[1,1, a c]]=[[a b, 1,1],[1,1, a], a c] \\
& =[a b,[1,[1, a, 1], 1], a c]=[[a b, 1,[1, a, 1]], 1, a c]=a b-{ }_{1} a+{ }_{1} a c
\end{aligned}
$$

where all the equalities follows by the one of distributivity, associativity, Mal'cev identities or Lemma 1.1.19(1). Thus $B$ is a skew-brace.

Corollary 2.1.12. A brace-type near-truss $T$ is associated with two-sided brace if and only if $T$ is a truss.

Remark 2.1.13. Observe that since the identity is a unique element in a truss, the assignment of a skew brace to a truss is unique.

Now, let us focus on trusses. Let us start with examples.

Example 2.1.14. A fundamental example of a (unital) truss is the endomorphism truss of an Abelian heap, $E(H)=\mathbf{A} \mathbf{h}(H, H)$, which has the pointwise defined heap operation and multiplication given by the composition of morphisms.

Lemma 2.1.15. Trusses form a category with trusses as objects and homomorphisms as arrows. We will denote this category by Trs. Moreover, unital trusses form a subcategory of Trs with unital trusses as objects and homomorphisms which preserve identities as arrows. We will denote this subcategory by $\operatorname{Trs}_{1}$.

Proof. This is a simple observation that composition of two truss homomorphisms is a truss homomorphism and that, if both homomorphisms preserve identity then the composition preserves the identity too. 
Remark 2.1.16. Observe that $\operatorname{Trs}_{1}$ is not a full subcategory of $\operatorname{Trs}$ as

$$
\operatorname{Trs}(\{0\}, \mathrm{T}(\mathbb{Z}))=\left\{f_{k}:\{0\} \rightarrow \mathrm{T}(\mathbb{Z}) \mid f(0)=k, k \in\{0,1\} \subset \mathbb{Z}\right\}
$$

is not equal to

$$
\operatorname{Trs}_{1}(\{0\}, \mathrm{T}(\mathbb{Z}))=\{f \mid f(0)=1\} .
$$

In an obvious way, the terminal object $\{*\}$ (that is, the singleton set with the unique ternary operation) of the category $\mathrm{Ah}$ is also the terminal object of the category Trs of trusses and the zero object (both initial and terminal) of the category $\operatorname{Trs}_{1}$ of unital ones. The empty set is a initial object in Trs.

Remark 2.1.17. The category $\operatorname{Trs}_{1}$ has kernels as every object has an identity and a homomorphism $\varphi$ of unital trusses preserves identity, we have a unique choice of a kernel, i.e. $\operatorname{ker}_{1}(\varphi)$, which is a unital truss and a sub-truss of an object.

Lemma 2.1.18. An assignment $\mathrm{T}:$ Ring $\rightarrow$ Trs given by $R \mapsto \mathrm{T}(R)$ and $\mathrm{T}(\varphi)=\varphi$, for all rings $R$ and all $\varphi \in \operatorname{Ring}\left(R, R^{\prime}\right)$, is a functor.

Proof. Let $\varphi \in \operatorname{Ring}(R, S)$, then for all $a, b, c \in R$,

$$
\begin{aligned}
\mathrm{T}(\varphi)[a, b, c] & =\varphi(a-b+c)=\varphi(a)-\varphi(b)+\varphi(c)=[\varphi(a), \varphi(b), \varphi(c)] \\
& =[\mathrm{T}(\varphi)(a), \mathrm{T}(\varphi)(b), \mathrm{T}(\varphi)(c)],
\end{aligned}
$$

thus $\mathrm{T}(\varphi) \in \operatorname{Trs}(\mathrm{T}(R), \mathrm{T}(S))$. Now, let $\varphi \in \operatorname{Ring}(R, S)$ and $\psi \in \operatorname{Ring}\left(S, S^{\prime}\right)$, then for all $a \in R$,

$$
\mathrm{T}(\psi) \circ \mathrm{T}(\varphi)(a)=\mathrm{T}(\psi)(\varphi(a))=(\psi \circ \varphi)(a)=\mathrm{T}(\psi \circ \varphi)(a),
$$

so an assignment $\mathrm{T}$ preserves composition and in consequence $\mathrm{T}$ is a functor.

It is worth to mention that analogous categories and functors can be constructed for pre-trusses and near-trusses but to keep some kind of common sense, I decided to limit my categorical considerations to trusses. 


\subsection{Congruences on pre-trusses}

In this section we will focus on the interpretation of congruences on pre-trusses as algebraic structures. This part is a natural extension of Section 1.2 into pre-trusses. The only and most important theorem of this section is Theorem 2.2.6 which identifies congruences with paragons and vice versa.

Definition 2.2.1. Let $T$ be a pre-truss.

(1) A sub-heap $S$ of $T$ is said to be left-closed (resp. right-closed) if, for all $s, s^{\prime} \in S$ and $t \in T$,

$$
\left[t s^{\prime}, t s, s\right] \in S \quad\left(\text { resp. }\left[s^{\prime} t, s t, s\right] \in S\right)
$$

(2) A sub-heap $S$ that is left- and right-closed is said to be closed.

(3) A non-empty normal sub-heap $P$ of $T$ such that every equivalence class of the sub-heap relation $\sim_{P}$ is a closed (normal) sub-heap of $T$ is called a paragon.

Observe that Lemma 1.2 .13 implies that if $P$ is a paragon in a pre-truss $T$, then all the equivalence classes of $\sim_{P}$ are mutually isomorphic paragons as well.

Remark 2.2.2. In the case of a non-empty sub-heap $S$ the quantifier 'for all $s \in S$ ' in the definition of the left or right closure property 2.2.1 can be equivalently replaced by the existential quantifier. Indeed, assume that there exists $q \in S$ such that, for all $s^{\prime} \in S$ and $t \in T,\left[t s^{\prime}, t q, q\right] \in S$. Then, for all $s \in S$,

$$
\left[t s^{\prime}, t s, s\right]=\left[\left[\left[t s^{\prime}, t q, q\right], q, t q\right], t s, s\right]=\left[\left[t s^{\prime}, t q, q\right],[t s, t q, q], s\right] \in S
$$

by the associativity, Mal'cev's identities, Lemma 1.1.19 (1), and the fact that $S$ is a sub-heap. Similarly, the right closure property follows.

Lemma 2.2.3. A non-empty normal sub-heap $P$ of a pre-truss $T$ is a paragon if and only if, for all $a, b \in T$ and $p, e \in P$,

$$
[a[p, e, b], a b, e] \in P \quad \& \quad[[p, e, b] a, b a, e] \in P .
$$


Proof. By Lemma 1.2.13, the equivalence class of $b \in T$ is $\bar{b}=P_{e}^{b}=\{[p, e, b] \mid p \in P\}$, for all $e \in P$. Hence $\bar{b}$ is left-closed if and only if, for all $p \in P$ and $a \in T$, there exists $q \in P$ such that

$$
[a[p, e, b], a b, b]=[q, e, b]
$$

that is, if and only if

$$
[a[p, e, b], a b, e]=q \in P
$$

as required.

Corollary 2.2.4. A non-empty normal sub-heap $P$ of a near-truss $T$ is a paragon if and only if $P$ is left-closed and all equivalence classes of the induced sub-heap relation are right-closed. In particular $P$ is a paragon in a skew truss if and only if it is a closed normal sub-heap.

Proof. Since in a near-truss the left distributivity law holds, the left-closure property in Lemma 2.2.3 reduces to $[a p, a e, e] \in P$, that is, the left-closedness of $P$. In a skew truss the right-closure property is treated symmetrically.

Corollary 2.2.4 shows that, in the case of skew trusses (and hence trusses) the notion of a paragon introduced in Definition 2.2.1 reduces to the notion introduced in [16, Definition 3.15].

Lemma 2.2.5. Let $f: T \longrightarrow T^{\prime}$ be a morphism of pre-trusses.

(1) For all $z \in \operatorname{Im} f, f^{-1}(z)$ is a paragon in $T$. In particular, if $P^{\prime}$ is a paragon in $\operatorname{Im} f$, then $f^{-1}\left(P^{\prime}\right)$ is a paragon in $T$.

(2) If $P$ is a paragon in $T$ then $f(P)$ is a paragon in $\operatorname{Im} f$.

Proof. (1) By Lemma 1.2.10, $f^{-1}(z)$ is a normal sub-heap which is non-empty (since $z \in \operatorname{Im} f)$. For all $a, b \in T$ and $p, e \in f^{-1}(z)$,

$$
\begin{aligned}
f([a[p, e, b], a b, e]) & =[f(a)[f(p), f(e), f(b)], f(a) f(b), f(e)] \\
& =[f(a)[z, z, f(b)], f(a) f(b), z]=z
\end{aligned}
$$


since $f$ preserves multiplication and ternary operations, and by Mal'cev identities. Thus $[a[p, e, b], a b, e] \in f^{-1}(z)$. By the same arguments, $[[p, e, b] a, b a, e] \in f^{-1}(z)$. In view of Lemma 2.2 .3 this means that $f^{-1}(z)$ is a paragon.

Assume that $P^{\prime}$ is a paragon. That the pre-image of a normal sub-heap is a normal sub-heap follows by the standard group-theoretic arguments. Since $f$ preserves multiplication and heap operation, for all $a, b \in T$ and $p, q \in f^{-1}\left(P^{\prime}\right)$,

$$
\begin{aligned}
& f([a[p, q, b], a b, q])=[f(a)[f(p), f(q), f(b)], f(a) f(b), f(q)] \quad \& \\
& f([[p, q, b] a, b a, q])=[[f(p), f(q), f(b)] f(a), f(b) f(a), f(q)] .
\end{aligned}
$$

Since $P^{\prime}$ is a paragon, and $f(p), f(q) \in P^{\prime}$, both expressions are elements of $P^{\prime}$. Therefore, $[a[p, q, b], a b, q],[[p, q, b] a, b a, q] \in f^{-1}\left(P^{\prime}\right)$, and hence $f^{-1}\left(P^{\prime}\right)$ is a paragon.

Statement (2) is proven by similar arguments.

Theorem 2.2.6. Let $P$ be a non-empty normal sub-heap of a pre-truss $T$. Then the canonical heap map $\pi: T \rightarrow T / P$ is a homomorphism of pre-trusses if and only if $P$ is a paragon.

Proof. Assume that $\pi$ is a pre-truss homomorphism. Since $P=\pi^{-1}(\pi(P)), P$ is a paragon by Lemma 2.2 .5 .

For the proof of the opposite implication assume that $P$ is a paragon. Then $\sim_{P}$ is a congruence on the heap $T$, so we only need to show that this relation is a congruence on the pre-truss $T$ as well. Let $a, b \in T$ be such that $a \sim_{P} b$, so that $a, b \in \pi(b)$. Since $P$ is a paragon, for all $t \in T,[t a, t b, b] \in \pi(b)$. Hence, $[\pi(t b), \pi(t a), \pi(b)]=\pi(b)$, that is, $\pi(t b)=\pi(t a)$ or, equivalently, $t a \sim_{P} t b$. In the same way one can prove that $a \sim_{P} b$ implies at $\sim_{P} b t$ for all $t \in T$. Assume that $a \sim_{P} b$ and $c \sim_{P} d$. Then $a c \sim_{P} b c, b c \sim_{P} b d$ and $a c \sim_{P} b d$, since $\sim_{P}$ is an equivalence relation. Therefore, $\sim_{P}$ is a congruence and the canonical map $\pi: T \rightarrow T / P$ is a homomorphism of pre-trusses. This completes the proof.

Corollary 2.2.7. If $P$ is a paragon in a pre-truss $T$, then for all $a, b \in T, \tau_{a}^{b}(P)=P_{a}^{b}$ is a paragon in $T$ and $T / P=T / P_{a}^{b}$. 
Proof. Since $P$ is a normal sub-heap, the corollary follows by the Lemma 1.2 .13 and the definition of paragon.

Corollary 2.2.8. Let $N$ be a near-ring. Then $P \subseteq N$ is an equivalence class for a congruence on $N$ if and only if $P$ is a paragon in $\mathrm{T}(N)$.

Proof. Let us assume that $P$ is an equivalence class for a congruence on $N$, let $\bar{N}$ be the quotient near-truss with canonical homomorphism $\pi: N \longrightarrow \bar{N}$. Since $\pi$ is also a homomorphism of associated near-trusses, that is, $\pi: \mathrm{T}(N) \longrightarrow \mathrm{T}(\bar{N})$, and $P=\pi^{-1}(P), P$ is a paragon in $\mathrm{T}(N)$ by Lemma 2.2 .5 .

In the opposite direction, assume that $P$ is a paragon in $\mathrm{T}(N)$. Then there exists a near-truss homomorphism $\pi: \mathrm{T}(N) \rightarrow \mathrm{T}(N) / P$. Observe that the triple $\left(\mathrm{T}(N) / P,+_{\pi(e)}, \cdot\right)$, where $e$ is the neutral element of $N$, is a near-ring, since the image of a left absorber through a surjective near-truss homomorphism is a left absorber. Therefore $\pi$ is also a homomorphism of the retracted near-rings and $P$ is an equivalence class of a congruence given by $\pi$ as $P=\pi^{-1}(P)$.

Corollary 2.2.9. Let $R$ be a ring and $P$ be a paragon in $\mathrm{T}(R)$. Then $\tau_{p}^{0}(P)=P_{p}^{0}$ is an ideal in $R$, for all $p \in P$.

Proof. If $P$ is a paragon in $R$, then for any $p \in P, 0 \in P_{p}^{0}$. Observe that by lemmas 2.2.7 and $1.2 .9 P_{p}^{0}$ is a normal subgroup and for all $a \in R$ and $b \in P$,

$$
[a b, a 0,0]=a b-a 0+0=a b \quad \& \quad[b a, 0 a, 0]=b a-0 a+0=b a .
$$

Thus $\mathrm{G}(P ; 0)$ is an ideal in $R$.

Now, let us consider skew braces. All the basic definitions and facts about skew braces reader will find in the Appendix B. For an equivalent characterisation of skew braces in terms of near-trusses, check Lemma 2.1.11. A quotient of a skew brace is given by an ideal, see Definition B.2.29 and Proposition B.2.30. It is easy to prove that every homomorphism of skew braces, see Definition B.2.28, is also a homomorphism of associated unital near-trusses. The opposite observation is not that simple. If 
we consider a surjective homomorphism of near-trusses associated to skew braces or a homomorphism in $\operatorname{Trs}_{1}$ between near-trusses associated to skew braces, then this homomorphism is also a homomorphism of corresponding skew braces. This follows by the following lemma.

Lemma 2.2.10. Let $\mathrm{T}(B)$ be the near-truss associated to a skew brace $B$ (with the identity 1). Then $P$ is a paragon in $\mathrm{T}(B)$ if and only if, for all $p \in P$,

$$
P_{p}^{1}=\tau_{p}^{1}(P)=\left\{\left[p^{\prime}, p, 1\right] \mid p^{\prime} \in P\right\}
$$

is an ideal in $B$.

Proof. Assume that $P$ is a paragon in $\mathrm{T}(B)$. Then $1 \in P_{p}^{1},\left(P_{p}^{1},+_{1}\right)$ is a normal subgroup of $(B,+)$ as $P_{p}^{1}$ is a normal sub-heap and $+_{1}=+$, see Lemma 1.2.9. Since $P_{p}^{1}$ is closed, for all $a \in B$ and $b \in P_{p}^{1}$,

$$
a b-a=[a b, a 1,1] \in P_{p}^{1} \quad \& \quad b a-a=[b a, 1 a, 1] \in P_{p}^{1} .
$$

Therefore, $b a-a b=c \in P_{p}^{1}$, and, using the brace distributive law,

$$
a^{-1} b a=a^{-1}(c+a b)=a^{-1} c-a^{-1}+b \in P_{p}^{1}
$$

since $P_{p}^{1}$ is left-closed. This implies that $a^{-1} P_{p}^{1} a=P_{p}^{1}$, that is, $a P_{p}^{1}=P_{p}^{1} a$, and completes the proof that $P_{p}^{1}$ is an ideal in $B$.

Conversely, if $P_{p}^{1}$ is an ideal in $B$, then $B / P_{p}^{1}$ is a brace by [27, Lemma 2.3] (see Lemma B.2.30, and the canonical brace epimorphism $\pi: B \longrightarrow B / P_{p}^{1}$ induces a neartruss morphism $\pi: \mathrm{T}(B) \longrightarrow \mathrm{T}\left(B / P_{p}^{1}\right)$. Since $P_{p}^{1}=\pi^{-1}\left(P_{p}^{1}\right), P_{p}^{1}$ and consequently also $P=\left(P_{p}^{1}\right)_{1}^{p}$ are paragons by Lemma 2.2 .5 .

Corollary 2.2.11. Let $B$ be a skew brace, then $P \subseteq B$ is an equivalence class for some congruence on $B$ if and only if $P$ is a paragon in $\mathrm{T}(B)$.

Proof. The proof of the left to right implication is the same as in Corollary 2.2.8. The other implication follows by Lemma 2.2.10. 
The next step is to prove that every near-truss homomorphism between near-trusses associated with skew braces is a skew brace homomorphism.

Lemma 2.2.12. Let $B_{1}$ and $B_{2}$ be skew braces. Then a near-truss homomorphism $f: \mathrm{T}\left(B_{1}\right) \rightarrow \mathrm{T}\left(B_{2}\right)$ is also a skew brace homomorphism.

Proof. Observe that by the Lemma 2.2.10 a map $f: B_{1} \rightarrow \operatorname{Im} f \subseteq B_{2}$ is a brace homomorphism. Now, the only difficulty is to show that the identity of $\operatorname{Im} f$ has the same identity as $B_{2}$. If $x$ is an idempotent in $\operatorname{Im} f$, then it is an idempotent in $B_{2}$, but there exists only one idempotent in $B_{2}$, the identity. Thus map $f$ preserves identity and is a homomorphism of skew braces.

Remark 2.2.13. The category of skew braces is isomorphic to a full subcategory of the category of near-trusses, i.e. the image of the functor T.

Example 2.2.14. View the ring $\mathbb{Z}$ as a truss with the heap operation $[k, l, m]_{+}=$ $k-l+m$ and the usual multiplication of integers. For any $n \in \mathbb{N}$, consider the ideal $n \mathbb{Z}$. Then, for all $m \in \mathbb{Z}$,

$$
(n \mathbb{Z})_{0}^{m}=\tau_{0}^{m}(n \mathbb{Z})=\{k n+m \mid k \in \mathbb{Z}\}=\{k n+r \mid k \in \mathbb{Z}\},
$$

where $r$ is a remainder of the division of $m$ by $n$, is a paragon. In particular if $n$ does not divide $m$, or, equivalently, $0<m<n$ then $(n \mathbb{Z})_{0}^{m}$ is not an ideal. One easily checks that

$$
\mathrm{T}(\mathbb{Z}) /(n \mathbb{Z})_{0}^{m} \cong \mathrm{T}(\mathbb{Z} / n \mathbb{Z})
$$

For example, $(2 \mathbb{Z})_{0}^{1}$ is the set of all odd integers but one can translate it to an ideal by taking $\left((2 \mathbb{Z})_{0}^{1}\right)_{1}^{0}=2 \mathbb{Z}$. In spite of the fact that $(2 \mathbb{Z})_{0}^{1}$ is not an ideal and that it contains the identity of $\mathbb{Z}$ it is a paragon different from $\mathbb{Z}$, with corresponding quotient being a non-trivial ring.

Example 2.2.15. Let $B$ be a two-sided brace. Following [28, Section 4] (see Definition B.2.31), the socle $\operatorname{Soc}(B)$ of $B$ is defined as

$$
\operatorname{Soc}(B)=\{a \in B \mid a b=a+b, b \in B\}
$$


The socle is an ideal of a two-sided brace that is non-trivial, i.e. different from $\{1\}$ if $B$ is non-trivial and finite (see [28, Proposition 3], [5] or Propostion B.2.33]). For all $c \in B$,

$$
c+\operatorname{Soc}(B)=\{c+a \mid a \in \operatorname{Soc}(B)\}
$$

is an equivalence class of a congruence on $B$ and hence it is a paragon in $\mathrm{T}(B)$. Indeed, $c+\operatorname{Soc}(B)$ is a sub-heap of $\mathrm{T}(B)$, since for all $a, a^{\prime}, a^{\prime \prime} \in \operatorname{Soc}(B)$,

$$
\left[c+a, c+a^{\prime}, c+a^{\prime \prime}\right]=c+a-c-a^{\prime}+c+a^{\prime \prime}=c+\left(a-a^{\prime}+a^{\prime \prime}\right) \in c+\operatorname{Soc}(B) .
$$

Furthermore, for all $b \in B$,

$$
[c, c b,(c+a) b]=c-c b+c b-b+a b=c+a b-b \in c+\operatorname{Soc}(B)
$$

and

$$
[b(c+a), b c, c]=b c-b+b a-b c+c=c+\left(b a b^{-1}\right) b-b \in c+\operatorname{Soc}(B),
$$

by the fact that $\operatorname{Soc}(B)$ is a normal subgroup of $(B, \cdot)$. Therefore, $c+\operatorname{Soc}(B)$ is closed, and hence it is a paragon in $\mathrm{T}(B)$.

Example 2.2.16. Let $R G$ be a group ring for an arbitrary ring $R$ and an arbitrary group $G$. Let us observe that, for all $r \in R$, the sets

$$
A_{r}:=\left\{\sum_{g \in G} r_{g} g \mid \sum_{g \in G} r_{g}=r\right\}
$$

are paragons in $\mathrm{T}(R G)$ as inverse images of $r$ under the ring (and hence truss) homomorphism

$$
\pi: R G \longrightarrow R, \quad \sum_{g \in G} r_{g} g \longmapsto \sum_{g \in G} r_{g}
$$

In particular, each $A_{r}$ is a sub-truss only if $r$ is an idempotent. It can be easily checked (or deduced from the fact that $\pi$ is an epimorphism combined with the first isomorphism theorem for algebras) that, for all $r \in R$,

$$
\mathrm{T}(R G) / A_{r} \cong \mathrm{T}(R)
$$




\subsection{Units from the perspective of trusses}

In this section, we will investigate when the set of units of a unital ring $R$ is a paragon in $\mathrm{T}(R)$. Even though the question is relatively simple, we only managed to acquire some necessary conditions. We conclude this section with Proposition 2.3.9, which identifies units of $\mathbb{Z}^{(2)} / 2^{k+1} \mathbb{Z}$ with Abelian cyclic braces, i.e. brace-type near-trusses which identity retracts are cyclic Abelian groups. All definitions and facts necessary to understand this section can be found in the Appendix B.

Lemma 2.3.1. Let $R G$ be a group ring. If the set of units $U(R G)$ of a ring $R G$ is a paragon in $\mathrm{T}(R G)$, then $U(R)$ is a paragon in $\mathrm{T}(R)$.

Proof. If $e$ is the neutral element of $G$, then it is easy to check that re $\in U(R G)$ if and only if $r \in U(R)$. In view of this observation and from the fact that $U(R G)$ is a paragon, if $r, r^{\prime}, r^{\prime \prime} \in U(R)$, then $\left[r e, r^{\prime} e, r^{\prime \prime} e\right]=\left[r, r^{\prime}, r^{\prime \prime}\right] e \in U(R G)$, which implies that $\left[r, r^{\prime}, r^{\prime \prime}\right] \in U(R)$. Hence $U(R)$ is a sub-heap of $\mathrm{T}(R)$. Furthermore, for all $r \in R$, and $r^{\prime}, r^{\prime \prime} \in U(R)$

$$
U(R G) \ni\left[r r^{\prime \prime} e, r r^{\prime} e, r^{\prime} e\right]=\left[r r^{\prime \prime}, r r^{\prime}, r^{\prime}\right] e
$$

which implies that $\left[r r^{\prime \prime}, r r^{\prime}, r^{\prime}\right] \in U(R)$. Similarly, $\left[r^{\prime \prime} r, r^{\prime} r, r^{\prime}\right] \in U(R)$. Therefore, $U(R)$ is a paragon in $\mathrm{T}(R)$.

Now, one can ask when units are a congruence class. Although at this stage we are not able to provide sufficient conditions we still can provide some necessary conditions.

Proposition 2.3.2. Let $R$ be a ring and assume that $U(R)$ is a paragon in $\mathrm{T}(R)$. Then

(1) For all $a, b \in U(R), a-b \notin U(R)$.

(2) Odd multiples of units in $R$ are units while even ones are not.

(3) The quotient truss $\mathrm{T}(R) / U(R)$ corresponds to a ring of characteristic 2.

Proof. (1) If $U(R)$ is a paragon in $\mathrm{T}(R)$ or, equivalently by Corollary 2.2.8 an equivalence class for a congruence on $R$, then its translate $U(R)_{b}^{0}$ is an ideal in $R$ (see Corollary 2.2.9. 
Explicitly, $U(R)_{b}^{0}$ consists of elements of the form $a-b$ where $a \in U(R)$. Since $0 \notin U(R)$, $U(R)_{b}^{0} \cap U(R)=\emptyset$ by Corollary 2.2.7 and hence $a-b \notin U(R)$.

(2) Note that for any $a \in U(R),-a \in U(R)$. We first prove by induction that $(2 n+1) a \in U(R)$, for all $n \in \mathbb{N}$. If $n=0$, then the statement is obvious. Now assume that $(2 n+1) a \in U(R)$, then

$$
(2 n+3) a=[(2 n+1) a,-a, a] \in U(R),
$$

so for all positive odd numbers and thus also the negative ones the assertion is true. Since any even multiple is a difference of two odd multiples, the second assertion follows by $(1)$.

(3) The equivalence class of $1 \in R, \overline{1}=U(R)$ is the identity in the quotient truss $\mathrm{T}(R) / U(R)$. Since both 1 and -1 are units in $R, \overline{-1}=\overline{1}$, which implies that $\overline{1}+\overline{1}=0$ in the ring corresponding to $\mathrm{T}(R) / U(R)$.

Corollary 2.3.3. Let $R$ be a ring with a finite characteristic $n \in \mathbb{N}$ and $U(R)$ be a congruence class, then 2 divides $n$.

Example 2.3.4. Let us consider ring $\mathbb{Z}_{4}$ and its associated truss $\mathrm{T}\left(\mathbb{Z}_{4}\right)$. It is easy to check that the set $U\left(\mathbb{Z}_{4}\right)=\{1,3\}$ is a paragon in $\mathrm{T}\left(\mathbb{Z}_{4}\right)$. Therefore, $U\left(\mathbb{Z}_{4}\right)$ is an element in the quotient of $\mathbb{Z}_{4}$ by the ideal $U\left(\mathbb{Z}_{4}\right)_{1}^{0}=\{1,3\}_{1}^{0}=\{0,2\}$, i.e. $\mathbb{Z}_{2}$.

The following theorem classifies all rings in which the set of units is a paragon and the quotient truss corresponds to the ring $\mathbb{Z}_{2}$.

Theorem 2.3.5. For a ring $R$, the following statements are equivalent:

(1) The units $U(R)$ form a paragon in $\mathrm{T}(R)$ and $\mathrm{T}(R) / U(R) \cong \mathrm{T}\left(\mathbb{Z}_{2}\right)$.

(2) For all $r \in R$, either $r \in U(R)$ or $1-r \in U(R)$.

Proof. Assume first that the statement (1) holds. Since $\mathrm{T}(R) / U(R) \cong \mathrm{T}\left(\mathbb{Z}_{2}\right)$ there are two disjoint paragons in $\mathrm{T}(R), U(R)$ and $J$, covering the whole of $\mathrm{T}(R)$. Since $0 \notin U(R)$, $0 \in J$ and hence $J$ is an ideal. Since $1 \in U(R), U(R)=\tau_{0}^{1}(J)$, by Proposition 4.2.15. 
Take any $r \in R$, then either $r \in U(R)$ or $r \in J$, in which case also $-r \in J$ (as $J$ is an ideal) and $1-r=\tau_{0}^{1}(-r) \in U(R)$. Hence the statement (2) holds.

In the converse direction, the assumption (2) means in particular that $R$ is a local ring, i.e. the set of non-units, say $J$, is an ideal in $R$. Note that if $u \in U(R)$ and $r \in J$, then $u+r \in U(R)$, for should $u+r$ not be a unit, then $1-u-r$ would be a unit, hence not an element of $J$, which would contradict the fact that $J$ is an ideal, as $1-u-r \in J$.

Take any $u, v, w \in U(R)$. Then

$$
[u, v, w]=u-v+w=(u-1)+(1-v)+w \in U(R),
$$

by the preceding discussion, as $u-1,1-v \in J$ and $J$ is an ideal. Hence $U(R)$ is a sub-heap of $\mathrm{T}(R)$. Next, take any $r \in R$ and $u \in U(R)$. Then, by the same token

$$
[r u, r, 1]=r(u-1)+1 \in U(R) .
$$

Therefore, $U(R)$ is a paragon in $\mathrm{T}(R)$.

Finally, take any $r \in R$. If $r \in U(R)$, then its class $\bar{r} \in \mathrm{T}(R) / U(R)$ is equal to $U(R)=\overline{1}$. If $1-r \in U(R)$, then also $r-1 \in U(R)$, and

$$
r=[r-1,-1,0]=\tau_{-1}^{0}(r-1) \in \tau_{-1}^{0}(U(R)),
$$

so that $r \in \overline{0}$. Thus there are two classes $\overline{0}, \overline{1}$ and the corresponding ring is $\mathbb{Z}_{2}$.

Corollary 2.3.6. The set $U\left(\mathbb{Z}_{n}\right)$ is a paragon in $\mathrm{T}\left(\mathbb{Z}_{n}\right)$ if and only if $n=2^{k}$ for some $k \in \mathbb{N}$.

Proof. Since the quotient truss $\mathrm{T}\left(\mathbb{Z}_{n}\right) / U\left(\mathbb{Z}_{n}\right)$ must correspond to a ring of characteristic 2 , in the case studied it must correspond to $\mathbb{Z}_{2}$. Thus necessarily we are in the situation of Theorem 2.3.5. Hence $U\left(\mathbb{Z}_{n}\right)$ is a paragon in $\mathrm{T}\left(\mathbb{Z}_{n}\right)$ if and only if, for all $m \in \mathbb{Z}_{n}$ $\operatorname{gcd}(m, n)=1$ or $\operatorname{gcd}(1-m, n)=1$. This is equivalent to $n$ having only even prime factors as needed.

Example 2.3.7. (1) Let us consider the subring of $\mathbb{Q}$ of the form

$$
\frac{\mathbb{Z}}{2 \mathbb{Z}+1}:=\left\{\frac{n}{2 p+1} \mid n, p \in \mathbb{Z}\right\} \text {. }
$$


Observe that the set of all invertible elements of $\frac{\mathbb{Z}}{2 \mathbb{Z}+1}$ is

$$
U\left(\frac{\mathbb{Z}}{2 \mathbb{Z}+1}\right)=\mathrm{O}(\mathbb{Q}):=\left\{\frac{2 q+1}{2 p+1} \mid q, p \in \mathbb{Z}\right\} .
$$

Clearly, $\mathrm{O}(\mathbb{Q})$ is not a subring but one can easily check that $\mathrm{O}(\mathbb{Q})$ is a well-defined sub-truss (and also a paragon) of $\mathrm{T}(\mathbb{Q})$. The elements $x$ of $\frac{\mathbb{Z}}{2 \mathbb{Z}+1}$ have either an odd numerator, in which case they are invertible or an even numerator, in which case $1-x$ has an odd numerator, hence invertible. Note in passing that $\mathrm{O}(\mathbb{Q})$ is an example of a two-sided brace with operation $-+_{1}-:=[-, 1,-]$.

(2) Let $\mathbb{F}$ be a field and consider the local ring $R=\mathbb{F}[x] /\left(x^{n}\right)$. The polynomials with root 0 are nilpotent hence not invertible. On the other hand polynomials with a constant coefficient are invertible. Explicitly, if $p(x)=\alpha+q(x)$, where $q(x)$ is nilpotent and $\alpha \neq 0$, then

$$
p(x)^{-1}=\alpha^{-1}-\alpha^{-2}\left(q(x)+q(x)^{2}+\ldots+q(x)^{n-1}\right) .
$$

Hence $R$ satisfies assumptions of Theorem 2.3.5 and so the set of polynomials with a non-zero constant coefficient is a paragon, and the quotient truss corresponds to the $\operatorname{ring} \mathbb{Z}_{2}$.

(3) The situation described in the preceding example can be adapted to polynomial rings with coefficients in general commutative rings. Consider $R=Q[x] /\left(x^{n}\right)$. If $Q$ is not an integral domain, then $R$ does not necessarily have the property of Theorem 2.3.5. so neither that the units of $R$ form a paragon nor, in case they do, that the quotient paragon will be associated to $\mathbb{Z}_{2}$ is guaranteed. By the arguments similar to those in the proof of Lemma 2.3.1 one easily finds that if $U(R)$ is a paragon, then so is $U(Q)$. Using this information, we now specify $Q=\mathbb{Z}_{m}$, and then by Corollary 2.3.6, necessarily $m=2^{k}$. Units of $\mathbb{Z}_{2^{k}}[x] /\left(x^{n}\right)$ are exactly all polynomials $p(x)$ such that $p(0)$ is coprime with 2. Clearly, if $p(x)$ is a unit, then $p(0)$ must be a unit, hence coprime with 2 . In the converse direction, the formula (2.3.1) gives the inverse to any polynomial with the constant term that is a unit in $\mathbb{Z}_{2^{k}}$. Let $p(x)$ be any element of $\mathbb{Z}_{2^{k}}[x] /\left(x^{n}\right)$. If $p(0)$ is coprime with 2 , then $p(x)$ is a unit. Otherwise, $1-p(x)$ has a constant term 
coprime with 2, hence it is a unit. Thus assertions of Theorem 2.3.5 are satisfied and we conclude that $U\left(\mathbb{Z}_{2^{k}}[x] /\left(x^{n}\right)\right)$ is a paragon in $\mathrm{T}(R)$ and the quotient truss is associated to $\mathbb{Z}_{2}$.

Lemma 2.3.8. Let $T$ be a unital truss. If the set of units $U(T)$ is a sub-heap of $T$, then $U(T)$ is (the truss associated to) a two-sided brace.

Proof. Assume that $U(T)$ is a sub-heap and since $U(T)$ is a group with truss multiplication, $U(T)$ is a brace-type truss in which every element is invertible, and hence is the truss associated to a two-sided brace by Lemma 2.1.11.

We conclude this section with the derivation of Abelian cyclic braces of [29, Proposition 4] as quotients of a commutative truss by a paragon.

Proposition 2.3.9. Let a be a positive integer and let $\mathbb{Z}^{(a)}$ denote the commutative unital truss with the heap operation derived from the addition in $\mathbb{Z}$, and the multiplication,

$$
m \cdot n=\text { amn }+m+n, \quad \text { for all } m, n \in \mathbb{Z}
$$

see [16, Corollary 3.53].

(1) For all $N \in \mathbb{Z}_{+}$,

$$
N \mathbb{Z}=\{m N \mid m \in \mathbb{Z}\},
$$

is a paragon in $\mathbb{Z}^{(a)}$.

(2) For all $k \geq 1, \mathbb{Z}^{(2)} / 2^{k+1} \mathbb{Z}$ is a brace-type truss in which every element is a unit (hence a two-sided brace) and

$$
U\left(\mathbb{Z}^{(2)} / 2^{k+1} \mathbb{Z}\right)=C_{2} \oplus C_{2^{k}} .
$$

Proof. (1) $N \mathbb{Z}$ is an Abelian subgroup of $\mathbb{Z}$, hence a sub-heap of $\mathbb{Z}$. Note that 0 is the identity in $\mathbb{Z}^{(a)}$. Hence, for all $m \in \mathbb{Z}$ and $n N \in N \mathbb{Z}$,

$$
\begin{aligned}
m \cdot_{0}(n N) & =[a m n N+m+n N, m, 0] \\
& =a m n N+m+n N-m=(a m n+n) N \in N \mathbb{Z} .
\end{aligned}
$$


Since $0 \in N \mathbb{Z}$, the assertion follows.

(2) First, one easily proves by induction that, for all $m \in \mathbb{Z}$ and $n \in \mathbb{N}$,

$$
m^{\cdot n}=\frac{(a m+1)^{n}-1}{a}
$$

where $m^{\cdot n}$ means the $n$-th power with respect of the product $(2.3 .2)$ in $\mathbb{Z}^{(a)}$. Using formula 2.3 .3 one proves that, for all $k \geq 1$,

$$
m^{\cdot 2^{k}} \equiv 0 \bmod 2^{k+1}
$$

in $\mathbb{Z}^{(2)}$. Indeed, if $k=1$,

$$
m^{\cdot 2}=\frac{(2 m+1)^{2}-1}{2}=2 m(m+1) \equiv 0 \bmod 4 .
$$

Next note that

$$
m^{\cdot 2^{k+1}}=\frac{(2 m+1)^{2^{k+1}}-1}{2}=\frac{(2 m+1)^{2^{k}}-1}{2}\left((2 m+1)^{2^{k}}+1\right) .
$$

Hence if the first factor is divisible by $2^{k+1}$, then $m^{\cdot 2^{k+1}}$ is divisible by $2^{k+2}$, since the second factor is even. Thus the stated congruence relation follows for all $k$ by the principle of induction.

Since 0 is the identity in $\mathbb{Z}^{(2)}$, the congruence relation 2.3 .4 implies that every element in $\mathbb{Z}^{(2)} / 2^{k+1} \mathbb{Z}$ is a unit and that elements of $U\left(\mathbb{Z}^{(2)} / 2^{k+1} \mathbb{Z}\right)$ have order not greater that $2^{k}$, hence $U\left(\mathbb{Z}^{(2)} / 2^{k+1} \mathbb{Z}\right)$ is not a cyclic group. We will show that 1 has the maximal order $2^{k}$. Since

$$
1^{2^{k}}=\frac{3^{2^{k}}-1}{2}
$$

this is equivalent to the statement that 3 is an order $2^{k}$ element in the group of units $U\left(\mathbb{Z}_{2^{k+2}}\right)$. This follows from the (inductively proven) fact that, for all $k$,

$$
3^{2^{k}}=1+n_{k} 2^{k+2}
$$

where $n_{k}$ is odd and the observation that the order of any element of $U\left(\mathbb{Z}_{2^{k+2}}\right)$ is a power of 2 .

Thus, $U\left(\mathbb{Z}^{(2)} / 2^{k+1} \mathbb{Z}\right)$ is an Abelian group of order $2^{k+1}$ that is not a cyclic group, but contains an element of order $2^{k}$, hence it must be isomorphic to $C_{2} \oplus C_{2^{k}}$. 


\section{Chapter 3}

\section{From trusses to braces}

The aim of this chapter is to describe a connection between skew braces and near-trusses. A "perfect" structure for a ring, and therefore for a truss with an absorber, is a field, as all elements besides absorber are invertible. In this chapter, we show that braces are exactly the same for trusses without absorbers as fields are for trusses with an absorber. It turns out that many results related to rings and fields can be rewritten without relying on the property of absorber. We conclude this chapter with a localisation of regular trusses. This chapter is a part of an article [26], Section 3.1] is a half of Section 3 of the paper, Section 3.2 is Section 4 and Section 3.3 is Section 5 .

\subsection{Near-trusses and braces}

In this chapter we will investigate when a quotient of a unital near-truss can be associated with a skew brace. To connect quotients of near-trusses with skew braces we need to determine which paragons do not produce absorbers in the quotients. To this end we introduce the notion of an ideal.

Definition 3.1.1. A normal sub-heap $I$ of a pre-truss $T$ is called a left (resp. right) $i d e a l$ if, for all $t \in T$ and $i \in I, t i \in I$ (resp. it $\in I$ ). If $I$ is both left and right ideal, then it is called an ideal. A left (resp. right) ideal is said to be maximal if it is not contained in any left (resp. right) proper ideal.

Note that an ideal is a closed sub-heap, but this does not yet make it into a paragon 
(see Definition 2.2.1), since the equivalence classes of the corresponding sub-heap relations need not be closed. Also note that if $f: T \rightarrow T^{\prime}$ is a homomorphism of pre-trusses, then the pre-image of an ideal in $\operatorname{Im} f$ is an ideal in $T$ and the image of an ideal in $T$ is an ideal in $\operatorname{Im} f$.

Lemma 3.1.2. If a left-closed normal sub-heap of a pre-truss contains a left ideal, then it is a left ideal.

Proof. Let $P$ be a left-closed normal sub-heap of $T$, and let $I$ be a left ideal such that $I \subseteq P$. Then, for all $p \in P, t \in T$ and $i \in I, t p=[[t p, t i, i], i, t i] \in P$, since $[t p, t i, i] \in P$ and $t i, i \in I \subseteq P$.

Lemma 3.1.3. Let $T$ be a pre-truss and $P$ be a paragon. Then $T / P$ has a left absorber (i.e. there exists $\bar{a} \in T / P$ such that for all $\bar{b} \in T / P \bar{b} \bar{a}=\bar{a}$ ) if and only if there exist $a \in P$ and $t \in T$ such that $P_{a}^{t}=\tau_{a}^{t}(P)$ (see Lemma 1.2.13) is a left ideal.

Proof. The assertion follows from the fact that for every $a \in P$ and $t \in T, P_{a}^{t}=\pi(t)$, where $\pi$ is the canonical surjection onto the quotient $T / P$.

Corollary 3.1.4. If I is a paragon that is a right ideal in a pre-truss $T$, then for all $e \in T \backslash I$ and all $a \in I, I_{a}^{e}$ is not a left ideal.

Proof. We know from Lemma 1.2 .13 that $T / I=T / I_{a}^{e}$. Assume that $I$ is a right ideal and suppose that $I_{a}^{e}$ is a left ideal. Then, by Lemma 3.1.3, $I$ is a right absorber in $T / I$ and $I_{a}^{e}$ is a left absorber in $T / I_{a}^{e}$. Hence $I=I_{a}^{e}$. But $e \notin I$ and $e \in I_{a}^{e}$, which yields a required contradiction and completes the proof.

Proposition 3.1.5. Let $T$ be a unital near-truss.

(1) $T$ is a truss associated with a skew brace if and only if $T$ has exactly one left ideal.

(2) $T$ is a truss associated with a near-field if and only if $T$ has a left absorber and exactly two left ideals. 
Proof. (1) Assume that $T$ has exactly one left ideal. For all $x \in T$ the left ideal $T x:=\{t x \mid t \in T\}$ has to be the whole of $T$ (in particular if $T$ has at least two elements, then it has no left absorbers). Therefore, there exists $y \in T$ such that $y x=1$ and $y$ is a left inverse to $x$. As $x$ is an arbitrary element there exists $x^{\prime}$ such that $x^{\prime} y=1$. Thus $\left(x^{\prime} y\right) x=x$ and by associativity $x^{\prime}=x$. The conclusion is that $y$ is the two-sided inverse of $x$ and the monoid $(T, \cdot)$ is a group. Therefore, the near-truss $T$ is a brace-type near-truss by Lemma 2.1.11.

Conversely, suppose that $T=\mathrm{T}(B)$ for a skew brace $B$ and that there exists a left ideal $I \subsetneq \mathrm{T}(B)$. Observe that if $x \in I$, then $x^{-1} x=1 \in I$, therefore $I=T$. This contradicts the assumption that $I \neq T$. Thus $T$ has exactly one left ideal.

(2) Let us assume that $T$ has a left absorber and exactly two left ideals. Then there exists a near-ring $R$ such that $T=\mathrm{T}(R)$, to be precise $R$ is the retract $(\mathrm{G}(\mathrm{T}(R) ; e), \cdot)$, where $e$ is the left absorber. Seeking contradiction, suppose that $R$ is not a near-field. Then there exists a left ideal $\{e\} \neq I \subsetneq R$; but $I$ is also a left ideal of $\mathrm{T}(R)$, which contradicts with the assumption that $T$ has only two left ideals. Therefore, $R$ is a left near-field.

Assume that $T=\mathrm{T}(F)$, where $F$ is a left near-field, then 0 (the neutral element for the addition in $F$ ) is a left absorber in $T$. Suppose by contradiction that $\mathrm{T}(F)$ has a left ideal $\{0\} \neq I \subsetneq \mathrm{T}(F)$. Consider, for any $a \in I$ the ideal $I_{a}^{0}:=\{[b, a, 0] \mid b \in I\}$. The ideal $I_{a}^{0}$ is neither equal to $\{0\}$ nor to $T$, since the map $[-, a, 0]$ is a bijection. Furthermore, $I_{a}^{0}$ is an ideal in $F$, and hence $F$ is not a near-field. This contradicts with the assumption that $F$ is a near-field.

Lemma 3.1.6. Let $T$ be a near-truss. If $I$ is a paragon in $T$ that is a left maximal ideal, then $T / I$ has no ideals different from a singleton set and $T / I$.

Proof. Suppose that $\mathfrak{J} \neq T / I$ is a left ideal in $T / I$ that is not a singleton set. Since $I$ is a left absorber in $T / I$, for any element $J \in \mathfrak{J}, \mathfrak{J}_{J}^{I}$ is a left ideal in $T / I$ by the left distributive law. Hence, $\pi^{-1}\left(\mathfrak{J}_{J}^{I}\right)$ is a left ideal in $T$, where $\pi: T \longrightarrow T / I$ is the canonical surjection. Moreover, $I \subset \pi^{-1}\left(\mathfrak{J}_{J}^{I}\right)$, since $I \in \mathfrak{J}_{J}^{I}$. Therefore, since $I$ is 
left maximal, either $I=\pi^{-1}\left(\mathfrak{J}_{J}^{I}\right)$, and hence $\mathfrak{J}_{J}^{I}=\{I\}$, which implies that $\mathfrak{J}=\{J\}$, or $\pi^{-1}\left(\mathfrak{J}_{J}^{I}\right)=T$, which implies in turn that $\mathfrak{J}=T / I$. Thus both cases lead to a contradiction.

Although dividing by a paragon which is a left maximal ideal yields a near-truss without proper left ideals, this near-truss always has an absorber. Therefore it is never a brace-type near-truss. The most straightforward idea to generalise maximality to paragons leads us to the following definition:

Definition 3.1.7. Let $T$ be a pre-truss. A left-closed (resp. right-closed) normal sub-heap $P \subsetneq T$ is said to be maximal if it is not contained in any left-closed (resp. right-closed) sub-heap other than $T$. A paragon $P$ is said to be left maximal (resp. right-maximal, maximal) if it is a maximal left-closed (resp. right-closed, left- and right-closed) sub-heap.

Lemma 3.1.8. Let $T$ be a near-truss or a skew-truss and $P$ be a left-closed normal sub-heap. Then $P$ is maximal if and only if, for all $a \in P$ and $t \in T, P_{a}^{t}$ is a maximal left-closed normal sub-heap.

Proof. Note that by the normality of $P$ and the left distributive law, all the $P_{a}^{t}$ are left-closed normal sub-heaps. Seeking contradiction assume that $P$ is maximal and there exists $a \in P$ and $t \in T$ such that $P_{a}^{t}$ is not maximal. Then there exists a left-closed normal sub-heap $Q$ such that $P_{a}^{t} \subsetneq Q \subsetneq T$. Since $\tau_{a}^{t}$ is an isomorphism with the inverse $\tau_{t}^{a}$, this implies that $P \subsetneq Q_{t}^{a} \subsetneq T$. Hence $P$ is not maximal, contrary to the assumption.

The opposite implication is also easily deduced from the fact that $P=\left(P_{a}^{t}\right)_{t}^{a}$.

Remark 3.1.9. In the case of rings the notion of maximal ideals and maximal paragons coincide as every paragon $P$ in the ring can be associated with an ideal $P_{a}^{0}$ for any $a \in P$ and an absorber 0 .

Lemma 3.1.10. Let $T$ be a near-truss or a skew-truss and $P \subseteq T$ a left maximal paragon, then $T / P$ has no proper (i.e. different from singletons and the whole of $T / P$ ) left ideals. 
Proof. By the definition of maximality of $P, T / P$ has no proper left paragons. Therefore it has no proper left ideals as a left ideal is a left paragon.

Observe that by dividing a near-truss without left absorbers by a paragon which is left-maximal one obtains a near-truss associated with a skew brace. If the quotient is a skew brace, then it is a simple brace, that is, it has no ideals in the sense of sub-braces different from the skew brace itself and singleton subsets of it. Maximal paragons do not characterise all the quotients which are brace-type near-trusses (near-trusses which identity retracts are braces), since there exist skew braces that are non-simple.

Theorem 3.1.11. Let $T$ be a unital near-truss and $P$ be a paragon, and let $\pi_{P}: T \longrightarrow$ $T / P$ be the canonical epimorphism. Then $T / P$ is a brace-type near-truss (see Lemma 2.1.11) if and only if, for all left ideals $I \subsetneq T$ and $\bar{a} \in T / P, \pi_{P}^{-1}(\bar{a}) \nsubseteq I$.

Proof. Let us assume that $T / P$ is a brace-type near-truss. Observe that should $\pi_{P}^{-1}(\bar{a}) \subseteq$ $I$ for a left ideal $I$, then $\pi_{P}(I)$ would be a left ideal in $T / P$. Thus, $\pi_{P}(I)=T / P$, since $T / P$ is a brace-type near-truss. On the other hand, if $c \in T \backslash I$ then $\pi_{P}(c) \notin \pi_{P}(I)$. Indeed, should $\pi_{P}(c) \in \pi_{P}(I)$, then there would exist $i \in I$ and $p \in P$ such that $[c, i, p] \in P$. Thus, for all $a \in \pi_{P}^{-1}(\bar{a}),[c, i, a]=[[c, i, p], p, a] \in \pi_{P}^{-1}(\bar{a}) \subset I$ and $c \in I$. Therefore, $I=T$.

Now, assume that, for all left ideals $I \subsetneq T$ and $\bar{a} \in T / P, \pi_{P}^{-1}(\bar{a}) \nsubseteq I$ and $T / P$ is not a brace-type near-truss. Then there exists a left ideal $\mathfrak{J} \subsetneq T / P$. The pre-image $\pi_{P}^{-1}(\mathfrak{J}) \subsetneq T$ is a left ideal in $T$ and, obviously, for any $\bar{j} \in \mathfrak{J}, \pi_{P}^{-1}(\bar{j}) \subseteq \pi_{P}^{-1}(\mathfrak{J})$. This contradicts the assumption that, for all $\bar{a} \in T / P, \pi_{P}^{-1}(\bar{a}) \nsubseteq \mathfrak{J}$, so $T / P$ is a brace-type near-truss. The proof is completed.

Example 3.1.12. Let $B$ be a skew brace and $R$ a ring. One can consider the product near-truss $\mathrm{T}(B) \times \mathrm{T}(R)$ with operation given by $(b, r)\left(b^{\prime}, r^{\prime}\right)=\left(b b^{\prime}, r r^{\prime}\right)$, for all $(b, r),\left(b^{\prime}, r^{\prime}\right) \in \mathrm{T}(B) \times \mathrm{T}(R)$. It is easy to check that, for any ideal $I$ in $R, \mathrm{~T}(B) \times I$ is an ideal in $\mathrm{T}(B) \times \mathrm{T}(R)$ and that for any paragon $P$ in $\mathrm{T}(B), P \times I$ is a paragon in $\mathrm{T}(B) \times \mathrm{T}(R)$. Every paragon of the form $P \times \mathrm{T}(R)$ fulfills conditions in Theorem 3.1.11 and one easily finds that $(\mathrm{T}(B) \times \mathrm{T}(R)) /(P \times \mathrm{T}(R)) \cong \mathrm{T}(B) / P$ 
Example 3.1.13. Let $T=2 \mathbb{Z}+1$. The set $P=\left\{2^{n} m+1 \mid m \in T\right\} \subset T$ is a paragon and the quotient $T / P$ is a brace-type truss isomorphic to $U\left(\mathbb{Z} / 2^{n+1} \mathbb{Z}\right)$, the sub-truss of all units in the quotient ring $\mathbb{Z} / 2^{n+1} \mathbb{Z}$. To prove that this isomorphism holds it is first of all helpful to notice that $|T / P|=2^{n}=\left|U\left(\mathbb{Z} / 2^{n+1} \mathbb{Z}\right)\right|$. Indeed, there are as many classes in the quotient as the odd numbers between $2^{n} m+1$ and $2^{n}(m+2)+1$ (it is important to notice that, if $m$ is odd, then $m+1$ is even), so exactly $2^{n}$. Then the isomorphism is given by sending $\overline{2 m+1} \in T / P$ to $2 m+1 \bmod 2^{n+1}$ : this is evidently injective, so also surjective since the two sets have the same size, and it is easily proven to be a homomorphism.

\subsection{From a near-truss to a domain}

The aim of this section is to introduce the notion of a completely prime paragon. This, in analogy to the case of rings, should lead to a quotient pre-truss that is a domain, i.e. a pre-truss in which cancellation properties hold. After describing such paragons, the next step is to consider the Ore localisation for pre-trusses. By inverting all elements of a domain we should obtain a pre-truss without proper left ideals and with no absorbers, so if the distributive law holds this will be a near-truss associated with a skew brace. Let us start with the definition of a domain. When working with rings, there is always an absorber which in many cases allows for simplification of some conditions. Not all pre-trusses have an absorber (in fact, having brace applications in mind, we are particularly interested in those that do not have absorbers), so many of the well-known definitions need to be in some sense generalised or stated without involving any absorber. We begin with the definition of a regular element:

Remark 3.2.1. We denote by $T^{\mathrm{Abs}}$ a set of elements of a pre-truss $T$ which are neither left or right absorbers with tacit understanding that $T^{\mathrm{Abs}}=T$ when $T$ has no absorbers.

Definition 3.2.2. Let $T$ be a pre-truss. A non-absorber element $a \in T^{\mathrm{Abs}}$ is said to be left regular (resp. right regular) if, for all $b \neq c$,

$$
a b \neq a c \quad(\text { resp. } b a \neq c a) .
$$


If $a$ is both left and right regular element then it is said to be regular.

Observe that conditions 3.2.1 can be written in a way that makes them reminiscent of the closedness conditions 2.2.1 used in the definition of a paragon. The statement that $a c \neq a b$ is equivalent to saying that $[a c, a b, b] \neq b$. Similarly, $b a \neq c a$ is equivalent to say that $[c a, b a, b] \neq b$. This indicates that these conditions are closely related to the definition of paragon.

Lemma 3.2.3. Let $T$ be a near-truss. Then $a \in T$ is a left regular element if and only if there exists an element $c$ such that, for all $b \in T \backslash\{c\}$,

$$
a b \neq a c .
$$

Proof. If $a$ is left regular then, for all $c \in T$ and all $b \in T \backslash\{c\}$, the inequality 3.2.2 holds, which implies the existence of $c$.

Assume that there exists $c \in T$ such that, for all $b \neq c, a b \neq a c$. Thus $\left[a b, a c, a c^{\prime}\right]=$ $a\left[b, c, c^{\prime}\right] \neq a c^{\prime}$, for all $c^{\prime} \in T$. Note that, for all $c, c^{\prime} \in T$, the map

$$
\left[-, c, c^{\prime}\right]: T \backslash\{c\} \longrightarrow T \backslash\left\{c^{\prime}\right\}, \quad b \longmapsto\left[b, c, c^{\prime}\right]
$$

is a bijection. Therefore, for all $t \in T \backslash\left\{c^{\prime}\right\}$, at $\neq a c^{\prime}$. By the arbitrariness of $c^{\prime}, a$ is a left regular element. This completes the proof.

Lemma 3.2.4. Let $R$ be a ring. Then $a \in R$ is a regular element (see Definition B.2.6) if and only if a is a regular element in $\mathrm{T}(R)$.

Proof. The equivalence will be proven for left regularity only, the right regularity case in symmetric. Let us assume that $a \in R$ is a regular element. Then there is no $b \in R \backslash\{0\}$ such that $a b=0$. Thus, by Lemma 3.2.3, if $c=0$ in 3.2 .2 , then $a$ is a regular element in $\mathrm{T}(R)$, since $a$ is regular in $R$.

Suppose that $a$ is regular in $\mathrm{T}(R)$. Then $a b \neq a c$ implies $a(b-c) \neq 0$. Therefore, by substituting $b=t+c$, at $\neq 0$ for all $t \in R \backslash\{0\}$, which completes the proof. 
Now we are ready to introduce the definition of a domain in clear analogy with the usual notion for rings.

Definition 3.2.5. A pre-truss $T$ is called a domain if all elements of $T^{\mathrm{Abs}}$ are regular.

In view of Lemma 3.2.4, a ring $R$ is a domain (see Definition B.2.7) if and only if $\mathrm{T}(R)$ is a domain.

Lemma 3.2.6. A near-truss $T$ is a domain if and only if it satisfies the cancellation property, that is for all $a \in T^{\mathrm{Abs}}$ and $b, b^{\prime} \in T$, each one of the equalities $a b=a b^{\prime}$ or $b a=b^{\prime} a$ implies that $b=b^{\prime}$.

Proof. This follows immediately for the definitions of a regular element and a domain.

Definition 3.2.7. Let $T$ be a pre-truss. A non-empty paragon $P \subseteq T$ is said to be completely prime if, for all $p \in P, a, b, c \in T$,

$$
\begin{gathered}
{[a b, a c, p] \in P \Longrightarrow P_{p}^{a} \text { is an ideal or }[b, c, p] \in P} \\
\text { and } \\
{[b a, c a, p] \in P \Longrightarrow P_{p}^{a} \text { is an ideal or }[b, c, p] \in P .}
\end{gathered}
$$

Lemma 3.2.8. Let $T$ be a pre-truss and $P$ be a non-empty paragon. Then $P$ is completely prime if and only if, for all $p \in P$ and $t \in T, P_{p}^{t}$ is completely prime.

Proof. Let us assume that $P$ is a completely prime paragon and let $p \in P$ and $t \in T$. We know that $P_{p}^{t}$ is a paragon (see comment that follows Definition 2.2.1). Then, for all $a, b, c \in T$ and $q \in P,[a b, a c,[q, p, t]] \in P_{p}^{t}$ implies $[[a b, a c,[q, p, t]], t, p]=[a b, a c, q] \in P$, since $\left(P_{p}^{t}\right)_{t}^{p}=P$. Thus, $P_{q}^{a}$ is an ideal or $[b, c, q] \in P$. In view of $\left(P_{p}^{t}\right)_{[q, p, t]}^{a}=P_{q}^{a}$, the first option is equivalent to $\left(P_{p}^{t}\right)_{[q, p, t]}^{a}$ being an ideal and the second to $[b, c,[q, p, t]] \in P_{p}^{t}$. Hence $P_{p}^{t}$ fulfils the left condition to be a completely prime paragon. Analogously one can prove that $P_{p}^{t}$ satisfies the right condition. Therefore, $P_{p}^{t}$ is a completely prime paragon. 
Unsurprisingly, the distributive laws yield simplification of the definition of a completely prime paragon.

Lemma 3.2.9. Let $T$ be a skew truss and $P$ be a paragon. Then $P$ is completely prime if and only if there exists $p \in P$ such that, for all $a, d \in T$,

$$
\begin{aligned}
{[a d, a p, p] \in P \Longrightarrow } & P_{p}^{a} \text { is an ideal or } d \in P \\
& \text { and } \\
{[d a, p a, p] \in P \Longrightarrow } & P_{p}^{a} \text { is an ideal or } d \in P .
\end{aligned}
$$

Proof. It is sufficient to observe that, for every $b \in T,[b, c, p]$ can be substituted by some $d \in T$ since $\tau_{c}^{p}=[-, c, p]: T \longrightarrow T$ is a bijection with the inverse given by $\tau_{p}^{c}[-, p, c]: T \longrightarrow T$. Hence, if $b=[d, p, c], d=[[d, p, c], c, p]$, and so

$$
[a b, a c, p]=[a[d, p, c], a c, p]=[a d, a p, p] \&[b a, c a, p]=[[d, p, c] a, c a, p]=[d a, p a, p]
$$

by the distributive laws and the axioms of a heap. This completes the proof.

Lemma 3.2.10. If $P \subsetneq T$ is a completely prime paragon in a pre-truss $T$, then, for all $p \in P$ and for all left (right) absorbers $a, a^{\prime} \in T, P_{p}^{a}=P_{p}^{a^{\prime}}$.

Proof. Let $a$ be a left absorber. For all $b, c \in T$ and $p \in P,[b a, c a, p]=[a, a, p]=p \in P$, so $P_{p}^{a}$ is an ideal or $[b, c, p] \in P$. The second option is equivalent to $b \sim_{P} c$, for all $b, c \in T$. Observe, though, that since $P \neq T$, there exist $b, c \in T$ such that $b \chi_{P} c$. Therefore, $P_{p}^{a}$ is an ideal and $\bar{a} \in T / P$ is an absorber. From the fact that if a truss has an absorber then it has only one left absorber one concludes that $P_{p}^{a}=P_{p}^{a^{\prime}}$, for all left absorbers $a, a^{\prime}$.

Theorem 3.2.11. Let $T$ be a pre-truss. Then $P$ is a completely prime paragon if and only if $T / P$ is a domain.

Proof. We write $\bar{a}$ for the class of $a$ in $T / P$. The pre-truss $T / P$ is a domain if and only if, for all $\bar{a}, \bar{b}, \bar{c} \in T / P, \overline{a b}=\overline{a c}$ implies that $\bar{b}=\bar{c}$ or $\bar{a}$ is an absorber. The equality 
$\overline{a b}=\overline{a c}$ amounts to the existence of $p \in P$ such that $[a b, a c, p] \in P$. Observe that $\bar{b}=\bar{c}$ if and only if $[b, c, p] \in P$, and $\bar{a}$ is an absorber if and only if $P_{p}^{a}$ is an ideal. The proof proceeds analogously for the right cancellation property.

Remark 3.2.12. Every paragon in a near-truss $\mathrm{T}(B)$ associated with a skew brace $B$ is completely prime.

Corollary 3.2.13. Let $R$ be a ring. An ideal $I$ is completely prime (see Definition B.2.13) in $R$ if and only if $I$ is a completely prime paragon in $\mathrm{T}(R)$.

Proof. Let us assume that $I$ is a completely prime ideal in $R$. Then, for all $a, b \in R$ and absorber $0 \in I$,

$$
[a b, a 0,0]=a b \in I \Longrightarrow a \in I \text { or } b \in I
$$

Thus, if $a \in I$, then $I_{0}^{a}=I$ is an ideal, and hence $I$ is a completely prime ideal in $\mathrm{T}(R)$.

Conversely, assume that $I$ is a completely prime paragon in $\mathrm{T}(R)$. For all $a, b \in \mathrm{T}(R)$,

$$
a b=[a b, a 0,0] \in I \Longrightarrow I_{0}^{a} \text { is an ideal or } b \in I .
$$

Observe that $I_{0}^{a}$ is an ideal if and only if $a \in I$. Therefore, $I$ is a completely prime ideal in $R$. This completes the proof.

Lemma 3.2.14. Let $f: T \rightarrow T^{\prime}$ be a morphism of pre-trusses. If $P$ is a completely prime paragon in $\operatorname{Im} f$, then $f^{-1}(P)$ is a completely prime paragon in $T$.

Proof. By Lemma 2.2.5, $f^{-1}(P)$ is a paragon. For all $a, b, c \in T$ and $p \in f^{-1}(P)$, if $[a b, a c, p] \in f^{-1}(P)$, then

$$
f([a b, a c, p])=[f(a) f(b), f(a) f(c), f(p)] \in P .
$$

This implies that $P_{f(p)}^{f(a)}$ is an ideal or $f([b, c, p])=[f(b), f(c), f(p)] \in P$. Therefore, $[b, c, p] \in f^{-1}(P)$ or $P_{f(p)}^{f(a)}$ is an ideal. Let us assume that

$$
z \in f^{-1}\left(P_{f(p)}^{f(a)}\right)=\{x \in T \mid \exists q \in P \text { s.t. } f(z)=[q, f(p), f(a)]\}
$$


Then $f(z)=[q, f(p), f(a)]$, for some $q \in P$ and $f([z, a, p])=[f(z), f(a), f(p)]=q \in P$. Hence $z=[[z, a, p], p, a] \in f^{-1}(P)_{p}^{a}$ and $f^{-1}\left(P_{f(p)}^{f(a)}\right) \subseteq f^{-1}(P)_{p}^{a}$. Therefore, $f^{-1}(P)_{p}^{a} \subseteq$ $f^{-1}\left(P_{f(p)}^{f(a)}\right)$ and by Lemma 3.1.2. $f^{-1}(P)_{p}^{a}$ is an ideal. This completes the proof.

We conclude this section with an example of a completely prime paragon and the corresponding quotient domain.

Example 3.2.15. Let $O(x)$ be the set of all polynomials in $\mathbb{Z}[x]$ in which the sum of coefficients is odd. One can easily check that $O(x)$ is a sub-monoid of the multiplicative monoid $\mathbb{Z}[x]$ and a sub-heap of $\mathbb{Z}[x]$ with the standard operation $[p, q, r]=p-q+r$. All this means that $O(x)$ is a (commutative) truss.

Take any $t_{0}, t_{1} \in O(x)$ and define

$$
P\left(t_{0}, t_{1}\right):=\left\{p \in O(x) \mid\left(t_{1}-t_{0}\right) \text { divides }\left(p-t_{0}\right)\right\}
$$

Then $P\left(t_{0}, t_{1}\right)$ is a paragon in $O(x)$ and it is a completely prime paragon provided that $t_{1}-t_{0}$ is irreducible in $\mathbb{Z}[x]$.

Proof. Clearly, if $p-t_{0}, q-t_{0}$ and $r-t_{0}$ are divisible by $t_{1}-t_{0}$, then so is $[p, q, r]-t_{0}=$ $p-q+r-t_{0}$. Hence $P\left(t_{0}, t_{1}\right)$ is a sub-heap of $O(x)$. Note that $t_{0} \in P\left(t_{0}, t_{1}\right)$, and hence, for all $p \in P\left(t_{0}, t_{1}\right)$ and $q \in O(x)$,

$$
\left[q p, q t_{0}, t_{0}\right]-t_{0}=q p-q t_{0}=q\left(p-t_{0}\right)
$$

Therefore, $\left[q p, q t_{0}, t_{0}\right]=\left[p q, t_{0} q, t_{0}\right] \in P\left(t_{0}, t_{1}\right)$, which means that $P\left(t_{0}, t_{1}\right)$ is a paragon.

Now assume that $c=t_{1}-t_{0}$ is irreducible in $\mathbb{Z}[x]$, and take $a, b \in O(x)$ for which there exists $p \in P\left(t_{0}, t_{1}\right)$ such that $[a b, a p, p] \in P$, that is $c \mid a\left(b-t_{0}\right)$. Since $c$ is irreducible, then either $c \mid\left(b-t_{0}\right)$, in which case $b \in P$, or $c \mid a$, that is, there exists $q \in \mathbb{Z}[x]$ such that $a=c q$. In this case,

$$
P\left(t_{0}, t_{1}\right)_{p}^{a}=\left\{r-p+c q \mid r \in P\left(t_{0}, t_{1}\right)\right\}
$$

Thus $P_{p}^{a}$ contains all elements of $O(x)$ divisible by $c$ (since $c \mid(r-p)$, for all $r, p \in$ $\left.P\left(t_{0}, t_{1}\right)\right)$, and hence it is an ideal in $O(x)$. Combined with the commutativity of $O(x)$, Lemma 3.2 .9 yields that $P\left(t_{0}, t_{1}\right)$ is a completely prime paragon. 
Note that in general in the situation described in Example 3.2.15.

$$
\bar{a}=\bar{b} \in O(x) / P\left(t_{0}, t_{1}\right) \text { if and only if } \quad\left(t_{1}-t_{0}\right) \mid(a-b) .
$$

So, for example, take $t_{0}=x$ and $t_{1}=x^{2}+x+1$. Then $c=t_{1}-t_{0}=x^{2}+1$ is an irreducible polynomial in $\mathbb{Z}[x]$ and $O(x) / P\left(x, x^{2}+x+1\right)$ is a domain that can be identified with the sub-truss $O(i)$ of the truss (ring) of Gaussian integers $\mathbb{Z}[i]$, defined as

$$
O(i)=\{m+n i \mid m+n \text { is odd }\}
$$

\subsection{A skew brace of fractions}

To summarise, up to now we have introduced the notions of a domain and a completely prime paragon, so that as long as we start with a pre-truss that has a completely prime paragon we can quotient out by it and obtain a domain. The next, and most important step, is to introduce localisation for pre-trusses. As the main goal of this chapter is to produce braces from near-trusses we will consider near-trusses without left absorbers and we will focus on localisation in the entire near-truss (to construct a "brace of fractions") following Ore's classic construction [30]. More about Ore localisation one can find in [31]. First observe that since not every ring can be localised the same is true for trusses. Following [30] we start by defining a regular pre-truss.

Definition 3.3.1. A pre-truss $T$ is said to be left regular if $T$ is a domain and it satisfies the left Ore condition, that is, for all $x, y \in T^{\mathrm{Abs}}$ (see Remark 3.2.1), there exist $r, s \in T^{\mathrm{Abs}}$ such that $r x=s y$.

In other words, a pre-truss is left (resp. right) regular if and only if $T^{\mathrm{Abs}}$ is a left Ore set (see Definition B.1.3). Next, we define the fraction relation on $T^{\mathrm{Abs}} \times T$, by

$$
(b, a) \sim\left(b^{\prime}, a^{\prime}\right) \text { if and only if there exist } \beta, \beta^{\prime} \in T^{\mathrm{Abs}} \text {, s.t. } \beta b=\beta^{\prime} b^{\prime} \text { and } \beta a=\beta^{\prime} a^{\prime} \text {. }
$$

This is an equivalence relation by the same arguments as in [30, Section 2]. The equivalence class of $(b, a)$ is denoted by $\frac{a}{b}$ and called a fraction, and the quotient set $T^{\text {Abs }} \times T / \sim$ is denoted by $\mathrm{Q}(T)$. 
Theorem 3.3.2. (Ore localisation for regular pre-trusses) Let $T$ be a left regular pre-truss. Then $\mathrm{Q}(T)$ is a pre-truss with the following operations

(a) For all $\frac{a}{b}, \frac{a^{\prime}}{b^{\prime}}, \frac{a^{\prime \prime}}{b^{\prime \prime}} \in \mathrm{Q}(T)$, the ternary operation is defined by

$$
\left[\frac{a}{b}, \frac{a^{\prime}}{b^{\prime}}, \frac{a^{\prime \prime}}{b^{\prime \prime}}\right]:=\frac{\left[\beta_{1} a, \beta_{2} a^{\prime}, \beta_{3} a^{\prime \prime}\right]}{\beta_{1} b}=\frac{\left[\beta_{1} a, \beta_{2} a^{\prime}, \beta_{3} a^{\prime \prime}\right]}{\beta_{2} b^{\prime}}=\frac{\left[\beta_{1} a, \beta_{2} a^{\prime}, \beta_{3} a^{\prime \prime}\right]}{\beta_{3} b^{\prime \prime}},
$$

where $\beta_{1}, \beta_{2}, \beta_{3}$ are any elements of $T^{\mathrm{Abs}}$ such that $\beta_{1} b=\beta_{2} b^{\prime}=\beta_{3} b^{\prime \prime}$.

(b) For all $\frac{a}{b}, \frac{a^{\prime}}{b^{\prime}} \in \mathrm{Q}(T)$,

$$
\frac{a}{b} \cdot \frac{a^{\prime}}{b^{\prime}}:=\frac{\gamma a^{\prime}}{\gamma^{\prime} b},
$$

where $\gamma, \gamma^{\prime} \in T^{\text {Abs }}$ are such that $\gamma b^{\prime}=\gamma^{\prime} a$.

Furthermore, $\left(\mathrm{Q}(T)^{\mathrm{Abs}}, \cdot\right)$ is a group. We will call $\mathrm{Q}(T)$ the pre-truss of (left) fractions of $T$.

Proof. We follow closely the proof of [30, Theorem 1]. The multiplication of fractions (3.3.2) is defined in such a way that $\frac{a}{b}$ can be interpreted as $b^{-1} a$. Since it relies entirely on the properties of the semigroup $(T, \cdot)$, the arguments of the proof of [30, Theorem 1] (with no modification, apart from the conventions) yield that $(\mathrm{Q}(T), \cdot)$ is a semigroup.

It remains to be proven that $\mathrm{Q}(T)$ is a heap. In fact, by the Ore condition we may assume that all fractions in the definition of the ternary operation (3.3.1) on $\mathrm{Q}(T)$ have common denominator, so that

$$
\left[\frac{a}{b}, \frac{a^{\prime}}{b}, \frac{a^{\prime \prime}}{b}\right]=\frac{\left[\beta a, \beta a^{\prime}, \beta a^{\prime \prime}\right]}{\beta b},
$$

since in this case we can choose $\beta:=\beta_{1}=\beta_{2}=\beta_{3}$. Thus suffices it to prove that (3.3.3) is well-defined, as then the heap axioms for $T$ will imply the corresponding axioms for the derived operation 3.3.3. We proceed in two steps. At first, we show that the formula (3.3.3) does not depend on the choice of $\beta$; in the second stage we will prove that it is also independent of the choice of the representatives $a, b$ for the class $\frac{a}{b}$.

Choose another element $s \in T^{\mathrm{Abs}}$ such that

$$
\left[\frac{a}{b}, \frac{a^{\prime}}{b}, \frac{a^{\prime \prime}}{b}\right]=\frac{\left[s a, s a^{\prime}, s a^{\prime \prime}\right]}{s b}
$$


There exist $g, g^{\prime} \in T^{\mathrm{Abs}}$ such that $g \beta b=g^{\prime} s b$, which implies

$$
g \beta=g^{\prime} s
$$

since $T$ is a domain. Therefore,

$$
g\left[\beta a, \beta a^{\prime}, \beta a^{\prime \prime}\right]=g^{\prime}\left[s a, s a^{\prime}, s a^{\prime \prime}\right], \quad g \beta b=g^{\prime} s b .
$$

Consequently,

$$
\frac{\left[\beta a, \beta a^{\prime}, \beta a^{\prime \prime}\right]}{\beta b}=\frac{\left[s a, s a^{\prime}, s a^{\prime \prime}\right]}{s b}
$$

which shows the independence of the formula (3.3.3) of the the choice of $\beta$.

To prove that the ternary operation 3.3.1 does not depend on the choice of the representatives in each equivalence class, let $(b, a),\left(b^{\prime}, a^{\prime}\right),\left(b^{\prime \prime}, a^{\prime \prime}\right),(d, c),\left(d^{\prime}, c^{\prime}\right),\left(d^{\prime \prime}, c^{\prime \prime}\right) \in$ $T^{\mathrm{Abs}} \times T$ be such that

$$
\frac{a}{b}=\frac{c}{d}, \frac{a^{\prime}}{b^{\prime}}=\frac{c^{\prime}}{d^{\prime}}, \frac{a^{\prime \prime}}{b^{\prime \prime}}=\frac{c^{\prime \prime}}{d^{\prime \prime}}
$$

and consider

$$
\left[\frac{a}{b}, \frac{a^{\prime}}{b^{\prime}}, \frac{a^{\prime \prime}}{b^{\prime \prime}}\right]=\frac{\left[\beta_{1} a, \beta_{2} a^{\prime}, \beta_{3} a^{\prime \prime}\right]}{\beta_{1} b}, \quad\left[\frac{a}{b}, \frac{a^{\prime}}{b^{\prime}}, \frac{c^{\prime \prime}}{d^{\prime \prime}}\right]=\frac{\left[s_{1} a, s_{2} a^{\prime}, s_{3} c^{\prime \prime}\right]}{s_{1} b}
$$

for suitable $\beta_{1}, \beta_{2}, \beta_{3}, s_{1}, s_{2}, s_{3} \in T^{\mathrm{Abs}}$. Then there exist $g, g^{\prime} \in T$, such that

$$
g \beta_{1} b=g \beta_{2} b^{\prime}=g \beta_{3} b^{\prime \prime}=g^{\prime} s_{1} b=g^{\prime} s_{2} b^{\prime}=g^{\prime} s_{3} d^{\prime \prime}
$$

and, since $T$ is a domain,

$$
g \beta_{1}=g^{\prime} s_{1}, g \beta_{2}=g^{\prime} s_{2}
$$

Thus both fractions in the equation (3.3.4 are equal if and only if $g \beta_{3} a^{\prime \prime}=g^{\prime} s_{3} c^{\prime \prime}$.

Observe, however, that since $g^{\prime} s_{3} d^{\prime \prime}=g \beta_{3} b^{\prime \prime}, g \beta_{3} a^{\prime \prime}=g^{\prime} s_{3} c^{\prime \prime}$ as $\frac{a^{\prime \prime}}{b^{\prime \prime}}=\frac{c^{\prime \prime}}{d^{\prime \prime}}$. Therefore,

$$
\left[\frac{a}{b}, \frac{a^{\prime}}{b^{\prime}}, \frac{a^{\prime \prime}}{b^{\prime \prime}}\right]=\left[\frac{a}{b}, \frac{a^{\prime}}{b^{\prime}}, \frac{c^{\prime \prime}}{d^{\prime \prime}}\right]
$$

The remaining equalities

$$
\left[\frac{a}{b}, \frac{a^{\prime}}{b^{\prime}}, \frac{c^{\prime \prime}}{d^{\prime \prime}}\right]=\left[\frac{a}{b}, \frac{c^{\prime}}{d^{\prime}}, \frac{c^{\prime \prime}}{d^{\prime \prime}}\right] \text { and }\left[\frac{a}{b}, \frac{c^{\prime}}{d^{\prime}}, \frac{c^{\prime \prime}}{d^{\prime \prime}}\right]=\left[\frac{c}{d}, \frac{c^{\prime}}{d^{\prime}}, \frac{c^{\prime \prime}}{d^{\prime \prime}}\right],
$$


are proven in a similar way. This completes the proof that the definition of the ternary operation (3.3.1) does not depend on the choice of representatives.

Finally, observe that if $a \in \operatorname{Abs}(T)$ then the class $\frac{a}{b}$ is an absorber and it is obviously unique. One can easily check that the class $\frac{b}{b}$ for $b \in T^{\mathrm{Abs}}$ is a neutral element of $\left(\mathrm{Q}(T)^{\mathrm{Abs}}, \cdot\right)$ and that if $a \notin T^{\mathrm{Abs}}$ then $\frac{a}{b}$ is a two-sided inverse to $\frac{b}{a}$. Thus $\left(\mathrm{Q}(T)^{\mathrm{Abs}}, \cdot\right)$ is a group. This completes the proof of the theorem.

From the fact that one can find a common denominator to any system of fractions one can observe that additional properties of $T$ are carried over to $\mathrm{Q}(T)$.

Proposition 3.3.3. Let $T$ be a regular pre-truss.

(1) If $T$ is Abelian, then so is $\mathrm{Q}(T)$.

(2) If $T$ is a near-truss, then $\mathrm{Q}(T)$ is a near-truss.

(3) If $T$ is a skew truss, then $\mathrm{Q}(T)$ is a skew-truss.

Proof. It is sufficient to consider heap operations of fractions with a common denominator, that is, those given by the formula (3.3.3). Statement (1) follows immediately from 3.3 .3 .

If $T$ is a near-truss, then

$$
\left[\frac{a}{b}, \frac{a^{\prime}}{b}, \frac{a^{\prime \prime}}{b}\right]=\frac{\left[\beta a, \beta a^{\prime}, \beta a^{\prime \prime}\right]}{\beta b}=\frac{\beta\left[a, a^{\prime}, a^{\prime \prime}\right]}{\beta b}=\frac{\left[a, a^{\prime}, a^{\prime \prime}\right]}{b} .
$$

Take any $\frac{c}{d}, \frac{a}{b}, \frac{a^{\prime}}{b}, \frac{a^{\prime \prime}}{b} \in \mathrm{Q}(T)$ and $\gamma, \gamma^{\prime} \in T^{\mathrm{Abs}}$ such that $\gamma b=\gamma^{\prime} c$, and compute

$$
\begin{aligned}
\frac{c}{d} \cdot\left[\frac{a}{b}, \frac{a^{\prime}}{b}, \frac{a^{\prime \prime}}{b}\right] & =\frac{c}{d} \cdot \frac{\left[a, a^{\prime}, a^{\prime \prime}\right]}{b}=\frac{\gamma\left[a, a^{\prime}, a^{\prime \prime}\right]}{\gamma^{\prime} d}=\frac{\left[\gamma a, \gamma a^{\prime}, \gamma a^{\prime \prime}\right]}{\gamma^{\prime} d} \\
& =\left[\frac{\gamma a}{\gamma^{\prime} d}, \frac{\gamma a^{\prime}}{\gamma^{\prime} d}, \frac{\gamma a^{\prime \prime}}{\gamma^{\prime} d}\right]=\left[\frac{c}{d} \cdot \frac{a}{b}, \frac{c}{d} \cdot \frac{a^{\prime}}{b}, \frac{c}{d} \cdot \frac{a^{\prime \prime}}{b}\right]
\end{aligned}
$$

Hence the left distributive law holds, and this proves statement (2).

To prove (3) we take $\frac{c}{d}, \frac{a}{b}, \frac{a^{\prime}}{b}, \frac{a^{\prime \prime}}{b} \in \mathrm{Q}(T)$ and $\gamma, \gamma^{\prime} \in T^{\mathrm{Abs}}$ such that $\gamma d=\gamma^{\prime}\left[a, a^{\prime}, a^{\prime \prime}\right]$. Then

$$
\left[\frac{a}{b}, \frac{a^{\prime}}{b}, \frac{a^{\prime \prime}}{b}\right] \cdot \frac{c}{d}=\frac{\left[a, a^{\prime}, a^{\prime \prime}\right]}{b} \cdot \frac{c}{d}=\frac{\gamma c}{\gamma^{\prime} b}
$$


On the other hand, using the definitions (3.3.1) and 3.3.2) and the right distributivity in $T$, we obtain

$$
\left[\frac{a}{b} \cdot \frac{c}{d}, \frac{a^{\prime}}{b} \cdot \frac{c}{d}, \frac{a^{\prime \prime}}{b} \cdot \frac{c}{d}\right]=\left[\frac{\gamma_{1} c}{\gamma_{1}^{\prime} b}, \frac{\gamma_{2} c}{\gamma_{2}^{\prime} b}, \frac{\gamma_{3} c}{\gamma_{3}^{\prime} b}\right]=\frac{\left[s_{1} \gamma_{1}, s_{2} \gamma_{2}, s_{3} \gamma_{3}\right] c}{s_{1} \gamma_{1}^{\prime} b}
$$

where $s_{1}, s_{2}, s_{3}, \gamma_{1}, \gamma_{2}, \gamma_{3}, \gamma_{1}^{\prime}, \gamma_{2}^{\prime}, \gamma_{3}^{\prime} \in T^{\text {Abs }}$ are such that

$$
\gamma_{1}^{\prime} a=\gamma_{1} b^{\prime \prime \prime}, \gamma_{2}^{\prime} a^{\prime}=\gamma_{2} b^{\prime \prime \prime}, \gamma_{3}^{\prime} a^{\prime \prime}=\gamma_{3} b^{\prime \prime \prime}, s_{1} \gamma_{1}^{\prime}=s_{2} \gamma_{2}^{\prime}=s_{3} \gamma_{3}^{\prime}
$$

Let $h, h^{\prime} \in T^{\mathrm{Abs}}$ be such that

$$
h \gamma^{\prime}=h^{\prime} s_{1} \gamma_{1}^{\prime}
$$

Then, using the distributive laws in $T,(3.3 .5$ and $(3.3 .6)$, we find

$$
\begin{aligned}
h \gamma d & =h \gamma^{\prime}\left[a, a^{\prime}, a^{\prime \prime}\right]=\left[h \gamma^{\prime} a, h \gamma^{\prime} a^{\prime}, h \gamma^{\prime} a^{\prime \prime}\right]=\left[h^{\prime} s_{1} \gamma_{1}^{\prime} a, h^{\prime} s_{1} \gamma_{1}^{\prime} a^{\prime}, h^{\prime} s_{1} \gamma_{1}^{\prime} a^{\prime \prime}\right] \\
& =h^{\prime}\left[s_{1} \gamma_{1}^{\prime} a, s_{2} \gamma_{2}^{\prime} a^{\prime}, s_{3} \gamma_{3}^{\prime} a^{\prime \prime}\right]=h^{\prime}\left[s_{1} \gamma_{1} d, s_{2} \gamma_{2} d, s_{3} \gamma_{3} d\right]=h^{\prime}\left[s_{1} \gamma_{1}, s_{2} \gamma_{2}, s_{3} \gamma_{3}\right] d
\end{aligned}
$$

The right cancellation property yields

$$
h \gamma=h^{\prime}\left[s_{1} \gamma_{1}, s_{2} \gamma_{2}, s_{3} \gamma_{3}\right]
$$

which in view of 3.3 .6 implies that

$$
\frac{\gamma c}{\gamma^{\prime} b}=\frac{\left[s_{1} \gamma_{1}, s_{2} \gamma_{2}, s_{3} \gamma_{3}\right] c}{s_{1} \gamma_{1}^{\prime} b}
$$

Therefore, also the right distributive law holds in the near-truss $\mathrm{Q}(T)$.

The construction of the truss of quotients is universal in the following sense.

Proposition 3.3.4. Let $T$ be a regular pre-truss. Then

(1) For any $b \in T^{\mathrm{Abs}}$,

$$
\iota_{b}: T \longrightarrow \mathrm{Q}(T), \quad a \longmapsto \frac{b a}{b},
$$

is a monomorphism of semigroups, and it is a monomorphism of trusses provided $T$ is a near-or skew-truss. 
(2) If $T$ is a unital pre-truss then $\iota_{1}$ is a monomorphism of unital trusses. Furthermore, for any brace-type near-truss $B$ and any unital truss homomorphism $f: T \longrightarrow B$, there exists a unique unital truss homomorphism $\hat{f}: \mathrm{Q}(T) \longrightarrow B$ rendering commutative the following diagram:

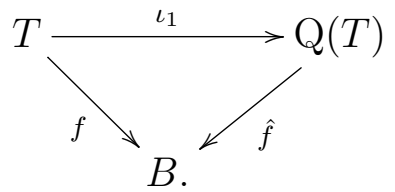

Proof. (1) Since $T$ is regular, $\iota_{b}$ is an injective map. For all $a, a^{\prime} \in T$,

$$
\iota_{b}\left(a a^{\prime}\right)=\frac{b a a^{\prime}}{b} \quad \& \quad \iota_{b}(a) \cdot \iota_{b}\left(a^{\prime}\right)=\frac{b a}{b} \cdot \frac{b a^{\prime}}{b}=\frac{\gamma b a^{\prime}}{\gamma^{\prime} b}
$$

where $\gamma, \gamma^{\prime}$ are such that $\gamma b=\gamma^{\prime} b a$. Take any $\beta, \beta^{\prime} \in T$ such that $\beta b=\beta^{\prime} \gamma^{\prime} b$. Then

$$
\beta b a a^{\prime}=\beta^{\prime} \gamma^{\prime} b a a^{\prime}=\beta^{\prime} \gamma b a^{\prime}
$$

which means that $\iota_{b}\left(a a^{\prime}\right)=\iota_{b}(a) \cdot \iota_{b}\left(a^{\prime}\right)$ as required.

In the case of a near- or skew-truss, that $\iota_{b}$ is a homomorphism of trusses follows by (3.3.3) and the left distributive law.

(2) The monomorphism of semigroups $\iota_{1}$ preserves the heap operation since 1 is the multiplicative identity in $T$.

Assume that $f: T \rightarrow B$ is a unital homomorphism of trusses and, for all fractions $\frac{a}{b} \in \mathrm{Q}(T)$, define

$$
\hat{f}: \mathrm{Q}(T) \longrightarrow B, \quad \frac{a}{b} \longmapsto f(b)^{-1} f(a) .
$$

This is well-defined since two fractions $\frac{a}{b}$ and $\frac{a^{\prime}}{b^{\prime}}$ are identical if and only if there are $\beta, \beta^{\prime}$ such that $\beta a=\beta^{\prime} a^{\prime}$ and $\beta b=\beta^{\prime} b^{\prime}$, in which case

$$
\begin{aligned}
\hat{f}\left(\frac{a}{b}\right) & =f(b)^{-1} f(a)=f(b)^{-1} f(\beta)^{-1} f(\beta) f(a) \\
& =f(\beta b)^{-1} f(\beta a)=f\left(\beta^{\prime} b^{\prime}\right)^{-1} f\left(\beta^{\prime} a^{\prime}\right)=f\left(b^{\prime}\right)^{-1} f\left(a^{\prime}\right)=\hat{f}\left(\frac{a^{\prime}}{b^{\prime}}\right),
\end{aligned}
$$

by the multiplicativity of $f$. By the same token, for all $\frac{a}{b}, \frac{a^{\prime}}{b^{\prime}} \in \mathrm{Q}(T)$,

$$
\hat{f}\left(\frac{a}{b} \cdot \frac{a^{\prime}}{b^{\prime}}\right)=\hat{f}\left(\frac{\gamma a^{\prime}}{\gamma^{\prime} b}\right)=f\left(\gamma^{\prime} b\right)^{-1} f\left(\gamma a^{\prime}\right)=f(b)^{-1} f\left(\gamma^{\prime}\right)^{-1} f(\gamma) f\left(a^{\prime}\right)
$$


where $\gamma, \gamma^{\prime} \in T$ are such that $\gamma b^{\prime}=\gamma^{\prime} a$. Applying $f$ to both sides of this equality and using the multiplicative property to $f$ we obtain

$$
f\left(\gamma^{\prime}\right)^{-1} f(\gamma)=f(a) f\left(b^{\prime}\right)^{-1}
$$

and hence

$$
\hat{f}\left(\frac{a}{b} \cdot \frac{a^{\prime}}{b^{\prime}}\right)=f(b)^{-1} f(a) f\left(b^{\prime}\right)^{-1} f\left(a^{\prime}\right)=\hat{f}\left(\frac{a}{b}\right) \hat{f}\left(\frac{a^{\prime}}{b^{\prime}}\right),
$$

that is $\hat{f}$ is a homomorphism of multiplicative groups. To check that $\hat{f}$ is a heap morphism it is enough to consider fractions with a common denominator and then

$$
\begin{aligned}
\hat{f}\left(\left[\frac{a}{b}, \frac{a^{\prime}}{b}, \frac{a^{\prime \prime}}{b}\right]\right) & =f(b)^{-1}\left[f(a), f\left(a^{\prime}\right) f\left(a^{\prime \prime}\right)\right] \\
& =\left[f(b)^{-1} f(a), f(b)^{-1} f\left(a^{\prime}\right) f(b)^{-1} f\left(a^{\prime \prime}\right)\right] \\
& =\left[\hat{f}\left(\frac{a}{b}\right), \hat{f}\left(\frac{a^{\prime}}{b}\right), \hat{f}\left(\frac{a^{\prime \prime}}{b}\right)\right],
\end{aligned}
$$

by the fact that $f$ is a heap homomorphism and the left distributive law in $B$. That $\hat{f} \circ \iota_{1}=f$ follows by the unitality of $f$.

Suppose that there exists a unital truss homomorphism $\hat{g}: \mathrm{Q}(T) \longrightarrow B$ such that $\hat{g} \circ \iota_{1}=f$. Note that

$$
\frac{a}{b}=\frac{1}{b} \cdot \frac{a}{1}
$$

In particular,

$$
1=\hat{g}\left(\frac{1}{1}\right)=\hat{g}\left(\frac{1}{b} \cdot \frac{b}{1}\right)=\hat{g}\left(\frac{1}{b}\right) f(b),
$$

where the last equality follows by the splitting assumption $\hat{g} \circ \iota_{1}=f$. Hence $\hat{g}\left(\frac{1}{b}\right)=$ $f(b)^{-1}$ and the equality $\hat{g}=\hat{f}$ follows by the multiplicativity of $\hat{g}$ and equations 3.3 .7 .

The following corollary provides one with the method of constructing skew braces, which might be considered as one of the main results of this chapter.

Corollary 3.3.5. If $T$ is a regular near-truss without an absorber, then $\mathrm{Q}(T)$ is a brace-type near-truss, that is, for all $b \in T$, the retract of $\mathrm{Q}(T)$ at $\frac{b}{b}$ with the product 3.3 .2 is a skew brace. 
Proof. Observe that if $T$ has no absorbers then $\mathrm{Q}(T)$ has no absorbers either. Indeed, suppose that there exists $\frac{a}{b} \in \mathrm{Q}(T)$ such that, for all $\frac{c}{d} \in \mathrm{Q}(T), \frac{c}{d} \cdot \frac{a}{b}=\frac{a}{b}$. Since $T$ has no absorbers, it has at least two elements, and hence, in particular we may consider $c \neq d$. Then there exist $\gamma, \gamma^{\prime} \in T$, such that $\frac{\gamma a}{\gamma^{\prime} d}=\frac{\gamma a}{\gamma b}$ and $\gamma^{\prime} c=\gamma b$. Thus $\frac{\gamma a}{\gamma^{\prime} d}=\frac{\gamma a}{\gamma^{\prime} c}$, so there exist $\beta, \beta^{\prime} \in T$ such that $\beta \gamma^{\prime} d=\beta^{\prime} \gamma^{\prime} c$ and $\beta \gamma a=\beta^{\prime} \gamma a$. By regularity, $\beta=\beta^{\prime}$ and $c=d$, which is the required contradiction. Therefore, $\frac{a}{b}$ is not an absorber for all $a, b \in T$. Now, since $\mathrm{Q}(T)$ is a group with multiplication and identity $\frac{b}{b}$, the retract of $\mathrm{Q}(T)$ in $\frac{b}{b}$ is a skew brace by Lemma 2.1.11

Note in passing that if $T$ satisfies the same assumptions as in Corollary 3.3.5. but there exists an absorber in $T$, then $\mathrm{Q}(T)$ is associated with a near-field.

Example 3.3.6. Let us consider $2 \mathbb{Z}+1$. It is a domain satisfying the Ore condition, thus it is a regular truss and we can localise it in itself. Since $2 \mathbb{Z}+1$ is commutative, the construction is much simpler than the one presented in the proof of Theorem 3.3.2. One can easily check that $\mathrm{Q}(2 \mathbb{Z}+1)=\frac{2 \mathbb{Z}+1}{2 \mathbb{Z}+1}:=\left\{\frac{2 p+1}{2 q+1} \mid p, q \in \mathbb{Z}\right\}$. The two-sided brace associated with this truss is the retract in 1 , i.e. the triple $(\mathrm{Q}(2 \mathbb{Z}+1),[-, 1,-], \cdot)$.

Similarly, the truss $O(x)$ of integer polynomials with coefficients summing up to odd numbers considered in Example 3.2.15 is regular with no absorbers, and hence it can be localised to a brace-type truss of the following rational functions

$$
\mathrm{Q}(O(x))=\frac{O(x)}{O(x)}:=\left\{\frac{p(x)}{q(x)} \mid p(x), q(x) \in O(x)\right\} .
$$

As a yet another example we can consider the truss $O(i)$ constructed as a special case of Example 3.2.15. Again this is a commutative domain satisfying the Ore condition and with no absorbers, and hence

$$
\begin{aligned}
\mathrm{Q}(O(i)) & =\left\{\frac{m+n i}{p+q i} \mid m+n \text { and } p+q \text { are odd integers }\right\} \\
& =\left\{\frac{m}{2 p+1}+\frac{n}{2 q+1} i \mid p, q \in \mathbb{Z}, m+n \text { is an odd integer }\right\} .
\end{aligned}
$$

The example of odd fractions described above is a special case of a more general construction. 
Example 3.3.7. Let $T_{n}(\mathbb{Z})$ denote the set of all $n \times n$-matrices over $\mathbb{Z}$ with odd entries on the diagonal and even off diagonal entries. That is,

$$
T_{n}(\mathbb{Z})=\left\{\left(a_{i j}\right)_{i, j=1}^{n} \mid a_{i i} \in 2 \mathbb{Z}+1 \& a_{i j} \in 2 \mathbb{Z}, i \neq j\right\} .
$$

(1) $T_{n}(\mathbb{Z})$ endowed with the matrix multiplication and the standard heap operation $[\mathbf{a}, \mathbf{b}, \mathbf{c}]=\mathbf{a}-\mathbf{b}+\mathbf{c}$ is a unital regular truss with no absorbers.

(2) The brace-type truss of fractions $\mathrm{Q}\left(T_{n}(\mathbb{Z})\right)$ can be identified with the set $T_{n}(\mathbb{Q})$ of $n \times n$-matrices over the rational numbers with diagonal entries made by the odd fractions (that is, fractions of both the numerator and denominator odd, $\mathrm{Q}(2 \mathbb{Z}+1))$ and with fractions with even numerator and odd denominator as off-diagonal entries. That is,

$$
\mathrm{Q}\left(T_{n}(\mathbb{Z})\right) \cong T_{n}(\mathbb{Q}):=\left\{\left(q_{i j}\right)_{i, j=1}^{n} \mid q_{i i} \in \frac{2 \mathbb{Z}+1}{2 \mathbb{Z}+1} \& q_{i j} \in \frac{2 \mathbb{Z}}{2 \mathbb{Z}+1}, i \neq j\right\} .
$$

It is clear that the set $T_{n}(\mathbb{Z})$ is closed under the described heap operation. That it is closed also under the matrix multiplication follows from an observation that in the product formula for the off-diagonal entries the sum involves the products of numbers of which at least one is even, while for the diagonal entry there is a single odd summand made out of the product of matching diagonal entries. Obviously $T_{n}(\mathbb{Z})$ has no absorber, as the zero matrix is not an element of $T_{n}(\mathbb{Z})$. Since the identity matrix has the prescribed form, $T_{n}(\mathbb{Z})$ is unital. The other statements of Example 3.3 .7 can be justified by the following (elementary) lemma.

Lemma 3.3.8. For all $\mathbf{a} \in T_{n}(\mathbb{Z})$,

(i) The determinant $\operatorname{det}(\mathbf{a})$ is an odd number.

(ii) The matrix of cofactors $\overline{\mathbf{a}}$ of $\mathbf{a}$ and hence also its transpose $\overline{\mathbf{a}}^{t}$ are elements of $T_{n}(\mathbb{Z})$.

Proof. Let $\mathbf{a}_{i, j}$ denote the matrix obtained from a by removing of the $i$-th row and the $j$-th column. Note that $\mathbf{a}_{i, i} \in T_{n-1}(\mathbb{Z})$ and that $\mathbf{a}_{i, j}, i \neq j$ has one row of even numbers. 
The first statement is proven by induction on the size $n$ of matrices. For $n=1$ the statement is obviously true. Assuming that the statement is true for $k$ we calculate the determinant of $\mathbf{a} \in T_{k+1}(\mathbb{Z})$ by expanding by the first row. Since $\mathbf{a}_{1,1}$ is an element of $T_{k}(\mathbb{Z}), \operatorname{det}\left(\mathbf{a}_{1,1}\right)$ is odd by inductive assumption. In the expansion of $\operatorname{det}(\mathbf{a})$ this is multiplied by the first entry $a_{11}$ of $\mathbf{a}$ and thus it gives an odd number. All the remaining summands involve products of other entries of the first row, which are even. Hence the sum of all terms in the expansion is odd as required.

The diagonal entries of $\overline{\mathbf{a}}$ are given by $\operatorname{det}\left(\mathbf{a}_{i, i}\right)$ which are odd by statement (i). Off-diagonal entries $(-1)^{i+j} \operatorname{det}\left(\mathbf{a}_{i, j}\right)$ are even since one row of each of $\mathbf{a}_{i, j}, i \neq j$ consists entirely of even numbers. The transposition statement is obvious.

With this lemma at hand we can now prove that $T_{n}(\mathbb{Z})$ is a domain satisfying the Ore condition. Since we can embed $T_{n}(\mathbb{Z})$ into a ring of matrices, the statement $\mathbf{a b}=\mathbf{a c}$, for some $\mathbf{a}, \mathbf{b}, \mathbf{c} \in T_{n}(\mathbb{Z})$ is equivalent to the statement that $\mathbf{a}(\mathbf{b}-\mathbf{c})=0$, hence

$$
0=\mathbf{a}(\mathbf{b}-\mathbf{c})=\overline{\mathbf{a}}^{t} \mathbf{a}(\mathbf{b}-\mathbf{c})=\operatorname{det}(\mathbf{a})(\mathbf{b}-\mathbf{c}),
$$

which implies that $\mathbf{b}=\mathbf{c}$, since $\operatorname{det}(\mathbf{a}) \neq 0$ by Lemma 3.3.8(i). The regularity of the other side of each $\mathbf{a} \in T_{n}(\mathbb{Z})$ can be proven in a symmetric way.

To prove the Ore condition we take any $\mathbf{a}, \mathbf{b} \in T_{n}(\mathbb{Z})$ and set

$$
\mathbf{r}=\mathbf{a} \overline{\mathbf{b}}^{t} \quad \& \quad \mathbf{s}=\operatorname{det}(\mathbf{b}) \mathbf{1} .
$$

Both these matrices are elements of $T_{n}(\mathbb{Z})$ by Lemma 3.3.8, and they satisfy the Ore condition $\mathbf{s a}=\mathbf{r b}$. Hence, $T_{n}(\mathbb{Z})$ is a left regular (in fact also right regular by similar arguments) truss.

For any element $\mathbf{q} \in T_{n}(\mathbb{Q})$ we write $q$ for the product of all denominators in entries of $\mathbf{q}$. This is an odd number and thus obviously $q \mathbf{q} \in T_{n}(\mathbb{Z})$. In particular, in view of Lemma 3.3.8, $\operatorname{det}(q \mathbf{q})$ is an odd number and its matrix of cofactors is an element of $T_{n}(\mathbb{Z})$. This in turn implies that the inverse of $\mathbf{q}$ is an element of $T_{n}(\mathbb{Z})$ divided by an odd number, hence an element of $T_{n}(\mathbb{Q})$. Consequently, $T_{n}(\mathbb{Q})$ is group with respect to 
multiplication of matrices. In order to identify $T_{n}(\mathbb{Q})$ with the truss of fractions $\mathrm{Q}\left(T_{n}(\mathbb{Z})\right)$ we will explore the universal property described in Proposition 3.3.4(2). Thus consider a brace-type skew truss $B$ and a homomorphism of unital trusses $f: T_{n}(\mathbb{Z}) \longrightarrow B$ and set

$$
\hat{f}: T_{n}(\mathbb{Q}) \longrightarrow B, \quad \mathbf{q} \longmapsto f(q \mathbf{1})^{-1} f(q \mathbf{q}) .
$$

Note that this definition does not depend on the way the fractions in $\mathbf{q}$ are represented, as the multiplication of the numerator and a denominator of an entry by a common (odd) factor results in multiplying both $q$ and $\mathbf{q}$ by the same factor which will cancel each other out in the formula for $\hat{f}$, by the multiplicative property of $f$. Since $q \mathbf{1}$ is a central element in $T_{n}(\mathbb{Z}), f(q \mathbf{1})^{-1}$ is central in the image of $f$ and, combined with the multiplicative property of $f$ this implies that $\hat{f}$ is a homomorphism of (multiplicative) groups. That $\hat{f}$ is a homomorphism of heaps follows by the distributivity. Obviously, $\hat{f} \circ \iota_{\mathbf{1}}=f$ and is a unique such morphisms. By the uniqueness of universal objects, $T_{n}(\mathbb{Q})$ is isomorphic to the truss of fractions $\mathrm{Q}\left(T_{n}(\mathbb{Z})\right)$. 


\section{Part III}

\section{Modules}





\section{Chapter 4}

\section{In the world of modules}

The purpose of this chapter is to introduce reader to modules over trusses. Every module $M$ over a ring $R$ is an example of a module over the associated truss, i.e. $\mathrm{H}(M)$ is a module over $\mathrm{T}(R)$ with action given by the action of $R$ on $M$. This chapter has four sections. In Section 4.1, we state basic properties of modules over trusses. In Section 4.2 . we introduce notion of an induced module and investigate quotients modules over trusses. In Section 4.3 , we explain how to introduce a $T$-action on a coproduct of heaps to obtain a coproduct of modules over trusses. In the last section, Section 4.4, we study monomorphisms and epimorphisms of $T$-modules. All the results of this chapter can be found in [19], [32], [33] and [16]. Most of the basic facts and definitions necessary for this chapter are in Appendix $A$.

\subsection{Modules over trusses}

In this section, we introduce a definition of a module over truss, give examples, and describe categories of modules over trusses.

Definition 4.1.1. Let $T$ be a truss. A left (right) $T$-module is an Abelian heap $M$ together with an associative left (right) action $\lambda_{M}: T \times M \longrightarrow M\left(\varrho_{M}: M \times T \longrightarrow M\right)$ of $T$ on $M$ that distributes over the heap operations. The action is denoted on elements by $t \cdot m=\lambda_{M}(t, m)\left(m \cdot t=\varrho_{M}(m, t)\right)$, with $t \in T$ and $m \in M$. Explicitly, the axioms 
of an action state that, for all $t, t^{\prime}, t^{\prime \prime} \in T$ and $m, m^{\prime}, m^{\prime \prime} \in M$,

$$
\begin{gathered}
t \cdot\left(t^{\prime} \cdot m\right)=\left(t t^{\prime}\right) \cdot m, \quad\left((m \cdot t) \cdot t^{\prime}=m \cdot t t^{\prime}\right) \\
{\left[t, t^{\prime}, t^{\prime \prime}\right] \cdot m=\left[t \cdot m, t^{\prime} \cdot m, t^{\prime \prime} \cdot m\right], \quad\left(m \cdot\left[t, t^{\prime}, t^{\prime \prime}\right]=\left[m \cdot t, m \cdot t^{\prime}, m \cdot t^{\prime \prime}\right]\right)} \\
t \cdot\left[m, m^{\prime}, m^{\prime \prime}\right]=\left[t \cdot m, t \cdot m^{\prime}, t \cdot m^{\prime \prime}\right], \quad\left(\left[m, m^{\prime}, m^{\prime \prime}\right] \cdot t=\left[m \cdot t, m^{\prime} \cdot t, m^{\prime \prime} \cdot t\right]\right)
\end{gathered}
$$

If $T$ is a unital truss and the action satisfies $1 \cdot m=m$, then we say that $M$ is a unital module.

Definition 4.1.2. Let $T$ and $S$ be trusses. A $T$-S-bimodule is a heap $M$ which is a left $T$-module and a right $S$-module such that for all $t \in T, s \in S$ and $m \in M$

$$
t \cdot(m \cdot s)=(t \cdot m) \cdot s .
$$

The T-T-bimodule $M$ will be called a two-sided $T$-module.

Remark 4.1.3. Equivalently, a (unital) T-module can be described as an Abelian heap $M$ together with a homomorphism of (unital) trusses $T \longrightarrow E(M)$, where $E(M)$ is an endomorphism truss.

Definition 4.1.4. A module homomorphism is a homomorphism of heaps between two modules that preserves actions. As it is customary in the ring theory we often refer to homomorphisms of $T$-modules as to $T$-linear maps or morphisms. The set of $T$-linear homomorphisms between $T$-modules $A$ and $B$ will be denoted by $\operatorname{Hom}_{T}(A, B)$.

Example 4.1.5. Every Abelian heap $H$ is a module over its endomorphism truss $E(H)$.

Lemma 4.1.6. Left (right) T-modules form a category. We denote this category by $T$-mod $(\bmod -T)$. Moreover, left (right) unital T-modules form a subcategory of T-mod $(\bmod -T)$, which we denote by $T_{1}-\bmod \left(\bmod -T_{1}\right)$.

Proof. Simply follows by the fact that a composition of $T$-module maps is a $T$-module map. 
Corollary 4.1.7. Let $T, S$ be trusses. Then $T$-S-bimodules form a category, which we denote by $T$-mod-S.

The terminal heap $\{*\}$ and the initial heap $\varnothing$, with the unique possible actions, are the terminal and the initial object, respectively, in all the foregoing categories. It is remarkable that, since $\{*\} \neq \varnothing$, all the categories do not have zero object.

Definition 4.1.8. An element $e$ of a left $T$-module $M$ is called an absorber, provided

$$
t \cdot e=e, \quad \text { for all } t \in T
$$

i.e. it is invariant under the $T$-action. The set of all absorbers of a module $M$ is denoted by $\operatorname{Abs}(M)=\{m \in M \mid t \cdot m=m, \forall t \in T\}$.

Lemma 4.1.9. A module homomorphism preserves absorbers.

Proof. Let $M, N$ be T-modules, $f: M \rightarrow N$ be a homomorphism of modules and $e \in M$ be an absorber. Then for all $t \in T$,

$$
t \cdot f(e)=f(t e)=f(e)
$$

so $f(e)$ is an absorber.

Example 4.1.10. An Abelian heap $M$ is a module over any truss $T$ with an action given for all $t \in T$ and $m \in M$ by

$$
t \cdot m=m \text {. }
$$

Example 4.1.11. A truss $2 \mathbb{Z}+1$ is a module over itself with action given by truss multiplication. This module has no absorber.

Corollary 4.1.12. Let $T$ be a truss with a left absorber 0 and $M$ be a left $T$-module. Then for all $m \in M, 0 m$ is an absorber.

Given a module $M$ over a ring $R$, one can consider a heap $\mathrm{H}(M)$ and a truss $\mathrm{T}(R)$. Observe that a truss $\mathrm{H}(M)$ is a $\mathrm{T}(R)$-module as for all $t, t^{\prime}, t^{\prime \prime} \in \mathrm{T}(R)$ and $m, m^{\prime}, m^{\prime \prime} \in M$,

$$
t\left[m, m^{\prime} m^{\prime \prime}\right]=t\left(m-m^{\prime}+m^{\prime \prime}\right)=t m-t m^{\prime}+t m^{\prime \prime}=\left[t m, t m^{\prime}, t m^{\prime \prime}\right]
$$




$$
\left[t, t^{\prime}, t^{\prime \prime}\right] m=\left(t-t^{\prime}+t^{\prime \prime}\right) m=t m-t^{\prime} m+t^{\prime \prime} m=\left[t m, t^{\prime} m, t^{\prime \prime} m\right]
$$

Therefore every module over a ring can be associated to a module over an associated truss. We will denote this module by $\mathrm{T}(M)$. Moreover, since every homomorphism of modules over rings is a homomorphism of modules over associated trusses i.e. $\mathrm{T}(\varphi)=\varphi$, for any homomorphism of $T$-modules, the assignment $\mathrm{T}: R$-mod $\rightarrow T$-mod is a functor from the category of modules over a ring to a category of modules over an associated truss. It is worth to mention that not every module over the truss associated with a ring is a module over this ring, see Example 4.1.10

Corollary 4.1.13. Let $M$ and $N$ be $\mathrm{T}(R)$-modules, for some ring $R$, with a unique absorbers $0_{M}, 0_{N}$, respectively. Then any $\mathrm{T}(R)$-module homomorphism $f: M \rightarrow N$ is also a $R$-module homomorphism between retracts $\mathrm{G}\left(M,+_{0_{m}}\right)$ and $\mathrm{G}\left(N,+_{0_{n}}\right)$.

Proof. The only thing to check is distributivity. The distributivity easily follows by Lemma 4.1 .12

Observe that a morphism of trusses $\varphi: T \longrightarrow S$ induces a change of scalars functor $S$-mod $\longrightarrow T$-mod: an $S$-module $M$ is a $T$-module with action $t \cdot m=\varphi(t) \cdot m$. In particular, any Abelian heap $H$ is a module over any truss $T$ through the action of $\{*\}$ on $H$ and the unique morphism $T \longrightarrow\{*\}$. Due to the unital action of $\{*\}$ in the case of modules, we will denote the terminal truss $\{*\}$ by $\{1\}$.

Given left $T$-modules $M$ and $N$ their product $M \times N$ has the left $T$-module structure defined component-wise, that is,

$$
\left[(m, n),\left(m^{\prime}, n^{\prime}\right),\left(m^{\prime \prime}, n^{\prime \prime}\right)\right]=\left(\left[m, m^{\prime}, m^{\prime \prime}\right],\left[n, n^{\prime}, n^{\prime \prime}\right]\right), \quad t \cdot(m, n)=(t \cdot m, t \cdot n)
$$

for all $t \in T, m, m^{\prime}, m^{\prime \prime} \in M$ and $n, n^{\prime}, n^{\prime \prime} \in N$.

The category of left (right or two-sided) $T$-modules is enriched over the category $(\mathbf{A h}, \times,\{1\})$ of abelian heaps. In particular, $\operatorname{Hom}_{T}(M, M) \subseteq \mathbf{A} \mathbf{h}(M, M)$ is a unital sub-truss of the unital endomorphism truss $E(M)$. We denote it by $E_{T}(M)$. We say that a functor $\mathrm{F}: T$-mod $\longrightarrow T^{\prime}$-mod is a heap functor if it induces a heap homomorphism 
between the hom-sets, that is, if for all $M, N \in T$-mod, the induced function

$$
\mathrm{F}_{M, N}: \operatorname{Hom}_{T}(M, N) \longrightarrow \operatorname{Hom}_{T}^{\prime}(\mathrm{F}(M), \mathrm{F}(N)), \quad \varphi \longmapsto \mathrm{F}(\varphi)
$$

is a homomorphism of Abelian heaps. Since functors preserve compositions and identities, $\mathrm{F}_{M, M}: E_{T}(M) \longrightarrow E_{T^{\prime}}(\mathrm{F}(M))$ is a morphism of unital trusses for all $M \in T$-mod.

\subsection{Congruences in modules}

The aim of this section is to investigate a quotient structure of a module over a truss.

Definition 4.2.1. Let $M$ be a left (right) $T$-module. Then a sub-heap $N \subseteq M$ is a left (right) submodule if for all $t \in T$ and $n \in N$,

$$
t \cdot n \in N, \quad(n \cdot t \in N) .
$$

If $M$ is a $T$-S-bimodule, then a sub-heap $N \subseteq M$ is a submodule if it is a left and right submodule of $M$.

Definition 4.2.2. Let $M$ be a left (right) $T$-module, $N$ be a sub-heap of $M$ and $e \in N$, then $N$ is called a left (right) $e$-induced submodule, if for all $t \in T \& n \in N$,

$$
t \triangleright_{e} n=[t n, t e, e] \in N, \quad\left(n_{e} \triangleleft t=[n t, e t, e] \in N\right) .
$$

We denote $N$ together with the operation $\triangleright_{e}$ by $N^{(e)}$.

Lemma 4.2.3. Let $N^{(e)}$ be a left (right) e-induced submodule of a T-module, then for all $m, n \in N, t \triangleright_{m} n \in N\left(n_{m} \triangleleft t \in N\right)$. The T-action $\triangleright_{m}\left({ }_{m} \triangleleft\right)$ is called a left (right) induced action.

Proof. Let $m, n \in N$, then

$$
\begin{aligned}
t \triangleright_{m} n & =[t m, t n, n]=[t m, t n, t e, t e, n]=[t[m, n, e], t e, n]=[t[m, n, e], t e, e, e, n] \\
& =\left[t \triangleright_{e}[m, n, e], e, n\right] \in N,
\end{aligned}
$$

as $t \triangleright_{e}[m, n, e], e, n \in N$ and $N$ is a sub-heap of $M$. The proof for the right induced action is the same. 
Definition 4.2.4. Let $M$ be a $T$-S-bimodule. A left $e$-induced submodule $N^{(e)} \subseteq M$ is an $e$-induced submodule if $N^{(e)}$ is also closed under a right induced action.

Lemma 4.2.5. Let $M$ be a left (right or two-sided) T-module, then an induced module $M^{(e)}$ is a left (right or two-sided) T-module.

Proof. Let $t, t^{\prime}, t^{\prime \prime} \in T$ and $m, m^{\prime}, m^{\prime \prime} \in M$, then

$$
\begin{aligned}
t \triangleright_{e}\left(t^{\prime} \triangleright_{e} m\right) & \left.=t \triangleright_{e}\left[t^{\prime} m, t^{\prime} e, e\right]=\left[t\left[t^{\prime} m, t^{\prime} e, e\right], t e, e\right]=\left[t t^{\prime} m, t t^{\prime} e, t e\right], t e, e\right] \\
& =\left[t t^{\prime} m, t t^{\prime} e, e\right]=t t^{\prime} \triangleright_{e} m,
\end{aligned}
$$

so 4.1.1a holds. Furthermore,

$$
\begin{aligned}
{\left[t, t^{\prime}, t^{\prime \prime}\right] \triangleright_{e} m } & =\left[\left[t m, t^{\prime} m, t^{\prime \prime} m\right],\left[t e, t^{\prime} e, t^{\prime \prime} e\right], e\right]=\left[[t m, t e, e],\left[t^{\prime} m, t^{\prime} e, e\right],\left[t^{\prime \prime} m, t^{\prime \prime} e, e\right]\right] \\
& =\left[t \triangleright_{e} m, t^{\prime} \triangleright_{e} m, t^{\prime \prime} \triangleright_{e} m\right], \\
t \triangleright_{e}\left[m, m^{\prime}, m^{\prime \prime}\right] & =\left[\left[t m, t m^{\prime}, t m^{\prime \prime}\right], t e, e\right]=\left[[t m, t e, e],\left[t m^{\prime}, t e, e\right],\left[t m^{\prime \prime}, t e, e\right]\right] \\
& =\left[t \triangleright_{e} m, t \triangleright_{e} m^{\prime}, t \triangleright_{e} m^{\prime \prime}\right],
\end{aligned}
$$

where in both equations second equalities follows by Lemma 1.1.19 4. Analogously one can check that in the case of right modules similar result holds.

Now, let us show that $M^{(e)}$ is a two-sided $T$-module. Let $t, t^{\prime} \in T$ and $m \in M$, then

$$
\begin{aligned}
\left(t \triangleright_{e} m\right)_{e} \triangleleft t^{\prime} & =[t m, t e, e]]_{e}^{\triangleleft} t^{\prime}=\left[[t m, t e, e] t^{\prime}, e t^{\prime}, e\right]=\left[t m t^{\prime}, t e t^{\prime}, e\right]=\left[\left[t m t^{\prime}, t e t^{\prime}, t e\right], t e, e\right] \\
& =\left[t\left(m_{e} \triangleleft t^{\prime}\right), t e, e\right]=t \triangleright_{e}\left(m_{e} \triangleleft t^{\prime}\right) .
\end{aligned}
$$

Therefore, indeed $M^{(e)}$ is a a left (right or two-sided) $T$-module.

Lemma 4.2.6. Let $M$ be a $T$-module. For any $e \in M$, if there exists a $\in M$ such that a is absorber in $M$ and $M^{(e)}$, then $e$ is an absorber.

If there exists an element $a \in M$ such that $a$ is an absorber in $M$ and $M^{(e)}$, then $e$ is an absorber in $M$.

Proof. Assume $a$ is an absorber in $M$ and $M^{(e)}$, then for all $t \in T$,

$$
t \triangleright_{e} a=[t a, t e, e]=a
$$

Now, since $a$ is an absorber in $M$, we get that $[a, t e, e]=a$. Therefore $t e=e$, so $e$ is an absorber in $M$. 
Corollary 4.2.7. If $e$ is not an absorber and $a$ is an absorber in $M$, then $a$ is not an absorber in $M^{(e)}$.

Lemma 4.2.8. Let $f: M \rightarrow N$ be a T-module map. Then for any $g \in \operatorname{Im}(f), \operatorname{ker}_{g}(f)$ is an induced submodule.

Proof. Let $g \in \operatorname{Im}(f)$, then for all $e \in M$ and $m \in \operatorname{ker}_{g}(f)$,

$$
f\left(t \triangleright_{e} m\right)=f([t m, t e, e])=[t f(m), t f(e), f(e)]=[t g, t g, g]=g,
$$

where the second equality follows by the property of $T$-module map and third by Mal'cev identities. Thus $\operatorname{ker}_{g}(f)$ is an induced submodule.

Theorem 4.2.9. Let $M$ be a $T$-module. If $N$ is a sub-heap of $M$, then the quotient $M / N$ has a T-module structure such that the canonical epimorphism $\pi: M \rightarrow M / N$ is a module morphism if and only if $N$ is an induced submodule of $M$.

Proof. Let us assume that $\pi$ is a canonical epimorphism, and $g \in \operatorname{Im}(\pi)$ is such that there exists $e \in N$ and $\pi(e)=g$. Then by Lemma 4.2.8, $N=\operatorname{ker}_{e}(\pi)$ is an induced submodule.

In the opposite direction, let us assume that $N$ is an induced submodule, then obviously $N$ is a normal sub-heap. Now, we can consider relation $\sim_{N}$ given by a normal sub-heap for all $m, m^{\prime} \in M$ by

$$
m \sim{ }_{N} m^{\prime} \Longleftrightarrow \exists e \in N\left[m, m^{\prime}, e\right] \in N
$$

If $m \sim_{N} m^{\prime}$, then

$$
\left[t m, t m^{\prime}, e\right]=\left[\left[t m, t m^{\prime}, t e\right], t e, e\right]=\left[t\left[m, m^{\prime}, e\right], t e, e\right]=t \triangleright_{e}\left[m, m^{\prime}, e\right]
$$

where second equality follows by Mal'cev identities and associativity, and third by the fact that $M$ is a $T$-module. Hence, as $\left[m, m^{\prime}, e\right] \in N$ and $N$ is an induced submodule, the relation $\sim_{N}$ is a congruence and $\pi$ is a canonical epimorphism of $T$-modules. 
Corollary 4.2.10. Any T-module homomorphism $\varphi: M \longrightarrow M^{\prime}$ factorizes uniquely as a T-linear map through the canonical epimorphism $M \longrightarrow M / N$ for any induced submodule $N$ of $M$ contained in $\operatorname{ker}_{e}(\varphi)$ as in Lemma 1.2.17.

Proof. The proof follows the same way as the proof of Lemma 1.2.17.

Lemma 4.2.11. If $N$ is a submodule of $M$, then $N$ is an induced submodule.

Proof. Indeed as for all $t \in T$ and $n, e \in N$,

$$
t \triangleright_{e} n=[t n, t e, e]
$$

but $t n, t e, e \in N$ and $N$ is a sub-heap, so $t \triangleright_{e} n \in N$. Thus $N$ is an induced submodule.

Lemma 4.2.12. If $N$ is a submodule of $M$, then $\pi(N)$, where $\pi$ is a canonical epimorphism, is an absorber in the quotient module $M / N$.

Proof. Let $\pi: M \rightarrow M / N$ be a canonical epimorphism, then for all $t \in T$,

$$
t \cdot \pi(N)=\pi(t \cdot N)=\pi(N)
$$

where second equality follows by the fact that $N$ is a submodule. Thus $\pi(N)$ is an absorber in $M / N$.

Theorem 4.2.13. Let $T$ be a truss, $M$ a T-module and $N$ a subset of $M$. Then the following statements are equivalent:

(1) $N$ is an induced submodule of $M$.

(2) $N$ is a sub-heap of $M$ and there is a congruence $\sim$ on $M$ such that $\pi_{N}=\pi_{\sim}$.

(3) $N$ is an equivalence class of a congruence on $M$.

Proof. (1) $\Longrightarrow(2)$ : If $N$ is an induced submodule of $M$, then the sub-heap relation $\sim_{N}$ is a congruence on $M$ by (the proof of) Theorem 4.2.9. 
$(2) \Longrightarrow(3)$ : Every induced submodule is a sub-heap and every sub-heap is an equivalence class for its sub-heap relation, see Lemma 1.2.11.

$(3) \Longrightarrow(1)$ : Assume that $N$ is an equivalence class for a congruence on $M$, say $\sim$. In particular $\sim$ is a congruence for the heap structure of $M$, hence $N$ is a sub-heap by [4, Theorem 1] (see Theorem 1.2.14). Furthermore, for all $t \in T$ and $n, n^{\prime} \in N$, $t \cdot n \sim t \cdot n^{\prime}$, i.e. $\pi_{\sim}(t \cdot n)=\pi_{\sim}\left(t \cdot n^{\prime}\right)$. Set $m=\left[t \cdot n, t \cdot n^{\prime}, n^{\prime}\right]$. Then

$$
\begin{aligned}
\pi_{\sim}(m) & =\pi_{\sim}\left(\left[t \cdot n, t \cdot n^{\prime}, n^{\prime}\right]\right) \\
& =\left[\pi_{\sim}(t \cdot n), \pi_{\sim}\left(t \cdot n^{\prime}\right), \pi_{\sim}\left(n^{\prime}\right)\right]=\left[\pi_{\sim}(t \cdot n), \pi_{\sim}(t \cdot n), \pi_{\sim}\left(n^{\prime}\right)\right] .
\end{aligned}
$$

Hence, $\pi_{\sim}(m)=\pi_{\sim}\left(n^{\prime}\right)$, i.e. $m \in N$. Thus $N$ is an induced submodule.

Theorem 4.2.13 can be applied to trusses associated to rings, thus yielding

Corollary 4.2.14. If $M$ is a module over a ring $R$, then $N \subseteq M$ is an equivalence class for a congruence $\sim$ on $M$ if and only if $N$ is an induced submodule of $\mathrm{T}(M)$.

Proof. Suffices it to observe that an equivalence relation is a congruence on $M$ as an $R$-module if and only if it is a congruence on $M$ as a $T(R)$-module and then apply Theorem 4.2.13.

Since every congruence relation of $R$-modules arises as the quotient by a submodule, Corollary 4.2 .14 gives interpretation of elements of quotients of $R$-modules $M / N$ as induced submodules of $\mathrm{T}(M)(M$ viewed as a module over the associated truss $\mathrm{T}(R))$. Furthermore, it provides one with the procedure of calculating the quotient of an $R$-module $M$ by the equivalence class of any element $m$ of $M$ : one simply needs to interpret $M$ as a heap and then take the quotient by the class of $m$ which is a sub-heap of $M$. In the same vein one obtains the following interpretation of elements of a quotient and hence of any ring.

The following proposition explains how to recover the submodule from any equivalence class in the quotient $R$-module $M / N$. 
Proposition 4.2.15. Let $M$ be a module over a truss $T$ and let $N$ be a left (resp. right) induced submodule of $M$. For all $e \in N$ and $m \in M$, consider the sub-heap

$$
N_{e}^{m}=\tau_{e}^{m}(N)=\{[n, e, m] \mid n \in N\}
$$

Then:

(1) $N_{e}^{m}$ is a left (resp. right) induced submodule.

(2) $N_{e}^{m}$ is a left (resp. right) submodule if and only if, for all $t \in T$,

$$
t \cdot m \in N_{e}^{m}, \quad\left(\text { resp. } m \cdot t \in N_{e}^{m}\right)
$$

(3) If $m \notin N$, then $N \cap N_{e}^{m}=\emptyset$.

Proof. (1) First let us note that, as a consequence of Mal'cev idenities, $m=\tau_{e}^{m}(e) \in N_{e}^{m}$. For all $t \in T$,

$$
\begin{aligned}
{[t \cdot[n, e, m], t \cdot m, m] } & =[[t \cdot n, t \cdot e, t \cdot m], t \cdot m, m] \\
& =[t \cdot n, t \cdot e, m]=[[t \cdot n, t \cdot e, e], e, m]=\tau_{e}^{m}([t \cdot n, t \cdot e, e]),
\end{aligned}
$$

by the (left) distributive law, associativity and Mal'cev identities. Since $N$ is a left induced module, $[t \cdot n, t \cdot e, e] \in N$, and so

$$
[t \cdot[n, e, m], t \cdot m, m] \in \tau_{e}^{m}(N)=N_{e}^{m}
$$

as required. The case of a right induced module is dealt with symmetrically.

(2) Obviously, if $N_{e}^{m}$ is a submodule and since $m \in N_{e}^{m}, t \cdot m \in N_{e}^{m}$. Conversely, if $t \cdot m \in N_{e}^{m}$, let $n_{m} \in N$ be such that

$$
t \cdot m=\tau_{e}^{m}\left(n_{m}\right)=\left[n_{m}, e, m\right]
$$

Then, for all $n \in N$,

$$
\begin{aligned}
t \cdot[n, e, m] & =[t \cdot n, t \cdot e, t \cdot m]=\left[t \cdot n, t \cdot e,\left[n_{m}, e, m\right]\right] \\
& =\left[\left[[t \cdot n, t \cdot e, e], e, n_{m}\right], e, m\right]=\tau_{e}^{m}\left(\left[[t \cdot n, t \cdot e, e], e, n_{m}\right]\right),
\end{aligned}
$$

by the (left) distributive law, associativity and the Mal'cev identities. Since $N$ is an induced submodule, $[t \cdot n, t \cdot e, e] \in N$. Consequently, $\left[[t \cdot n, t \cdot e, e], e, n_{m}\right] \in N$, and therefore $t \cdot \tau_{e}^{m}(n) \in \tau_{e}^{m}(N)=N_{e}^{m}$, for all $n \in N$ and $t \in T$, as required. 
(3) Suppose that $n \in N$ is an element of $N_{e}^{m}$, so that there is $n^{\prime} \in N$ such that $n=$ $\left[n^{\prime}, e, m\right]$. By the associativity of $[-,-,-]$ and the Mal'cev identities, $m=\left[n^{\prime}, e, n\right] \in N$, which contradicts the assumption that $m \notin N$.

Since $N_{e}^{m}$ is the image of $N$ under the heap isomorphism $\tau_{e}^{m}, N_{e}^{m} \cong N$ as heaps. By the foregoing proposition this isomorphism is an isomorphism of induced modules, therefore $M / N_{e}^{m} \cong M / N$. Moreover, since for all $a \in M, \bar{a} \in M / N_{e}^{m}$ and $\bar{a} \in M / N$ are equal as sets, one gets that $M / N_{e}^{m}=M / N$. In the case of a module $M$ over a ring $R$, an $R$-submodule is obtained from the element $N$ of the quotient module by choosing $m=0$, the zero of $M$.

\subsection{Coproduct of modules}

In this section, we introduce the coproduct of $T$-modules as a coproduct of underlying heaps with coordinatewise $T$-action. This section is a part of [19, Section 3].

Let $\left(A_{x}\right)_{x \in X}$ be a family of left modules over a truss $T$. By the distributivity of action, for each $t \in T$ and $x \in X$ the function $\lambda_{x}^{t}: A_{x} \longrightarrow \underset{x \in X}{\boxplus} A_{x}, a \longmapsto t \cdot a$, is a homomorphism of heaps. For each $t \in T$, the family $\left(\lambda_{x}^{t}\right)_{x \in X}$ extends to the homomorphism of heaps $\underset{x \in X}{\boxplus} \lambda_{x}^{t}: \underset{x \in X}{\boxplus} A_{x} \longrightarrow \underset{x \in X}{\boxplus} A_{x}$, and thus there is a $T$-action

$$
T \times \underset{x \in X}{\boxplus} A_{x} \longmapsto \underset{x \in X}{\boxplus} A_{x}, \quad(t, a) \mapsto \underset{x \in X}{\boxplus} \lambda_{x}^{t}(a),
$$

which makes $\underset{x \in X}{\boxplus} A_{x}$ into a $T$-module. This action is defined letter-by-letter, so for example in the case of a two-element family of $T$-modules $A$ and $B$,

$$
t \cdot \overline{: a_{1} b_{1} a_{2} \ldots a_{k} b_{k} a_{k+1}:}=\overline{:\left(t \cdot a_{1}\right)\left(t \cdot b_{1}\right)\left(t \cdot a_{2}\right) \ldots\left(t \cdot a_{k}\right)\left(t \cdot b_{k}\right)\left(t \cdot a_{k+1}\right)},
$$

where $t \in T, a_{i} \in A$ and $b_{i} \in B$, etc.

For $T$-modules $A, B$ we can explicitly write out what the module action looks like on $\mathrm{G}\left(A ; e_{A}\right) \oplus \mathrm{G}\left(B ; e_{B}\right) \oplus \mathbb{Z}$, by transferring it through the isomorphism $\varphi$ in Proposition 1.4.4. The action is given by the formula $t \star \varphi(x)=\varphi(t \cdot x), x \in A \boxplus B$, 
and, for all $a \in A, b \in B$ and $n \in \mathbb{Z}$, it comes out as

$$
t \star(a+b+n)=t \cdot a-n\left(t \cdot e_{A}\right)+t \cdot b+(n-1)\left(t \cdot e_{B}\right)+n,
$$

where the use of the additive notation tacitly presupposes that $e_{A}=e_{B}=0$ in the direct sum of Abelian groups $\mathrm{G}\left(A ; e_{A}\right) \oplus \mathrm{G}\left(B ; e_{B}\right) \oplus \mathbb{Z}$. In particular, in the case that both $t \cdot e_{B}=e_{B}$ and $t \cdot e_{A}=e_{A}$ the action takes the simple form $t \star(a, b, n)=(t \cdot a, t \cdot b, n)$.

\subsection{Monomorphisms and epimorhpisms}

Let $T$ be a truss. It will be useful in Chapters 6 and 7 to know that epimorphisms (respectively, monomorphisms) of $T$-modules are always effective, that is, that they are coequalizers (respectively, equalizers) of their kernel pairs (respectively, cokernel pairs), and that they coincide with surjective (respectively, injective) $T$-linear maps.

To this aim, recall that if $f: M \longrightarrow N$ is a morphism of $T$-modules, its kernel pair (respectively, cokernel pair) is the pullback (respectively, pushout) of the pair $(f, f)$, see Example A.0.25 and Example A.0.31.

Proposition 4.4.1. Every epimorphism of T-modules is surjective.

Proof. Assume that $M$ and $N$ are $T$-modules. If both $M$ and $N$ are the empty $T$ module, the empty map is an epimorphism (by uniqueness) and it is also surjective (trivially). If only $N$ is the empty module, then we cannot have morphisms from a non-empty to the empty module. If only $M$ is the empty module, then the empty map to $N$ is not an epimorphism. Summing up, we may assume that both $M$ and $N$ are non-empty and that $\varphi: M \longrightarrow N$ is an epimorphism of $T$-modules. Consider the $T$-submodule $\operatorname{Im}(\varphi) \subseteq N$ and the canonical projection $\pi: N \longrightarrow N / \operatorname{Im}(\varphi)$. For $p \in \operatorname{Im}(\varphi)$, consider also the constant morphism $\tau_{\bar{p}}: N \longrightarrow N / \operatorname{Im}(\varphi), n \longmapsto \bar{p}:=\pi(p)$. For every $m \in M, \varphi(m) \sim_{\operatorname{Im}(\varphi)} p$ and hence $\pi(\varphi(m))=\pi(p)=\bar{p}=\tau_{\bar{p}}(\varphi(m))$. Since $\varphi$ is an epimorphism, $\pi=\tau_{\bar{p}}$ and hence every $n \in N$ satisfies $n \sim_{\operatorname{Im}(\varphi)} p$ (that is, for all $\varphi(m) \in \operatorname{Im}(\varphi),[n, p, \varphi(m)] \in \operatorname{Im}(\varphi))$. In particular, $n=[n, p, p] \in \operatorname{Im}(\varphi)$ for all $n \in N$ and $\varphi$ is surjective. 
Proposition 4.4.2. Every epimorphism of T-modules is the coequalizer of its kernel pair.

Proof. Assume that $\pi: M \longrightarrow P$ is an epimorphism of $T$-modules. The kernel relation together with its coordinate projections

$$
\begin{gathered}
\operatorname{Ker}(\pi)=\left\{\left(m_{1}, m_{2}\right) \in M \times M \mid \pi\left(m_{1}\right)=\pi\left(m_{2}\right)\right\} \subseteq M \times M, \\
p_{i}: \operatorname{Ker}(\pi) \longrightarrow M, \quad\left(m_{1}, m_{2}\right) \longmapsto m_{i}, \quad i=1,2,
\end{gathered}
$$

yield the following fork of $T$-modules

$$
\operatorname{Ker}(\pi) \underset{p_{2}}{\stackrel{p_{1}}{\longrightarrow}} M \stackrel{\pi}{\longrightarrow} P \text {. }
$$

Assume that $f: M \longrightarrow N$ is any other $T$-module map such that $f \circ p_{1}=f \circ p_{2}$ and consider $\bar{f}: P \longrightarrow N$ given by $\bar{f}(\pi(m)):=f(m)$. The map $\bar{f}$ is well-defined because if $\pi\left(m_{1}\right)=\pi\left(m_{2}\right)$, then $\left(m_{1}, m_{2}\right) \in \operatorname{Ker}(\pi)$ and hence $f\left(m_{1}\right)=\left(f \circ p_{1}\right)\left(m_{1}, m_{2}\right)=$ $\left(f \circ p_{2}\right)\left(m_{1}, m_{2}\right)=f\left(m_{2}\right)$. It is a morphism of $T$-modules because $\pi$ and $f$ are $T$-linear maps. It is a unique morphism such that $\bar{f} \circ \pi=f$ because $\pi$ is an epimorphism. Thus, $(P, \pi)$ satisfies the universal property of the coequalizer of the pair $\left(p_{1}, p_{2}\right)$. To conclude, observe that $\left(\operatorname{Ker}(\pi), p_{1}, p_{2}\right)$ is the kernel pair of $f$.

Proposition 4.4.3. Every monomorphism of T-modules is injective.

Proof. Let $f: M \longrightarrow N$ be a monomorphism of $T$-modules. As before, there is a fork diagram of $T$-modules

$$
\operatorname{Ker}(f) \underset{p_{2}}{\stackrel{p_{1}}{\longrightarrow}} M \stackrel{f}{\longrightarrow} N .
$$

The fact that $f$ is a monomorphism implies that $p_{1}=p_{2}$ and hence $(m, n) \in \operatorname{Ker}(f)$ if and only if $m=n$, which in turn entails that $f(m)=f(n)$ if and only if $m=n$.

Lemma 4.4.4. Let $M, N$ be T-modules, $M \boxplus N$ their coproduct in $T$-mod and let $\iota_{M}: M \longrightarrow M \boxplus N, \iota_{N}: N \longrightarrow M \boxplus N$ be the structure maps of the coproduct. Then $\iota_{M}(m) \neq \iota_{N}(n)$ for all $m \in M, n \in N$. 
Proof. Endow the Abelian heap $\mathrm{H}\left(\mathbb{Z}_{2}\right)$ with the trivial $T$-module structure: $t \cdot x=x$ for all $t \in T$ and $x \in \mathbb{Z}_{2}$. The assignments

$$
\varphi_{M}: M \longrightarrow \mathrm{H}\left(\mathbb{Z}_{2}\right), m \longmapsto 0, \quad \text { and } \quad \varphi_{N}: N \longrightarrow \mathrm{H}\left(\mathbb{Z}_{2}\right), n \longmapsto 1
$$

are well-defined $T$-linear morphisms and hence they induce, by the universal property of the coproduct, a unique $T$-linear map $\Phi: M \boxplus N \longrightarrow \mathrm{H}\left(\mathbb{Z}_{2}\right)$ such that $\Phi \circ \iota_{M}=\varphi_{M}$ and $\Phi \circ \iota_{N}=\varphi_{N}$. If we suppose that there exist $m \in M$ and $n \in N$ such that $\iota_{M}(m)=\iota_{N}(n)$, then

$$
0=\varphi_{M}(m)=\Phi\left(\iota_{M}(m)\right)=\Phi\left(\iota_{N}(n)\right)=\varphi_{N}(n)=1
$$

which is a contradiction.

Proposition 4.4.5. Every monomorphism of T-modules is the equalizer of its cokernel pair.

Proof. Since the category of $T$-modules is cocomplete, i.e. it has all small colimits (by [17, Theorem 9.4.14] or Theorem C.0.16, for example), it is enough to prove that every monomorphism is regular, that is, that it is the equalizer of some pair of arrows.

Assume that $M$ and $N$ are $T$-modules. If $M$ is the empty $T$-module, then the empty map is a monomorphism (because there are no maps from a non-empty to the empty module) and it is also the equalizer of the pair

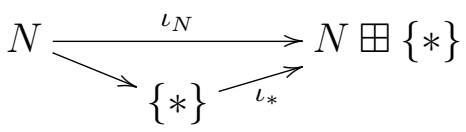

by Lemma 4.4.4. If $M$ is non-empty, then $N$ cannot be the empty module, since we cannot have morphisms from a non-empty to the empty module. Summing up, we may assume that both $M$ and $N$ are non-empty and that $f: M \longrightarrow N$ is a monomorphism of $T$-modules. Consider then $e^{\prime} \in M, N \supseteq \operatorname{Im}(f) \ni e=f\left(e^{\prime}\right)$, the quotient $T$-module $N / \operatorname{Im}(f)$, the absorber $\bar{e}=\operatorname{Im}(f)$ therein and the canonical projection $\pi: N \longrightarrow N / \operatorname{Im}(f)$. Then there is a fork diagram of $T$-modules

$$
M \stackrel{f}{\longrightarrow} N \underset{\tau_{\bar{e}}}{\stackrel{\pi}{\longrightarrow}} N / \operatorname{Im}(f),
$$


where $\tau_{\bar{e}}$ denotes the $T$-linear morphism sending everything to $\bar{e}$. Let us check that $(M, f)$ is the equalizer of the pair $\left(\pi, \tau_{\bar{e}}\right)$. If $P$ is another $T$-module and $g: P \longrightarrow N$ is a $T$-linear map such that $\pi(g(p))=\bar{e}$ for all $p \in P$, then this implies that there exists $f(m) \in \operatorname{Im}(f)$ such that

$$
\left[g(p), f\left(e^{\prime}\right), f(m)\right]=[g(p), e, f(m)] \in \operatorname{Im}(f) .
$$

In particular,

$$
g(p)=\left[\left[g(p), f\left(e^{\prime}\right), f(m)\right], f(m), f\left(e^{\prime}\right)\right] \in \operatorname{Im}(f),
$$

and hence there exists a (necessarily unique, in view of Proposition 4.4.3) element $m_{p} \in M$ such that $g(p)=f\left(m_{p}\right)$. Since, in addition,

$$
f\left(m_{t \cdot p}\right)=g(t \cdot p)=t \cdot g(p)=t \cdot f\left(m_{p}\right)=f\left(t \cdot m_{p}\right),
$$

for all $p \in P$ and $t \in T$, the assignment $h: P \longrightarrow M, p \longmapsto m_{p}$, is a $T$-linear morphism such that $f \circ h=g$ and it is unique satisfying this property, because $f$ is injective. Summing up, $(M, f)$ is indeed the equalizer of (4.4.1), as claimed.

Finally, since equalizers of Abelian heaps and $T$-modules are simply equalizers in Set endowed with the sub-heap or $T$-submodule structure, let us describe explicitly a construction of coequalizers in the categories of Abelian heaps and $T$-modules.

Lemma 4.4.6. Given a diagram

$$
A \underset{\psi}{\stackrel{\varphi}{\longrightarrow}} B
$$

in $\mathbf{A h}$ and any $e \in B$, define

$$
N(e)=\{[\varphi(a), \psi(a), e] \mid a \in A\} .
$$

Then

1. The set $N(e)$ is a sub-heap of $B$ and, for different choices of e, the heaps $N(e)$ are mutually isomorphic. 
2. Let $\overline{N(e)}:=\langle N(e), e\rangle$ be the sub-heap of $B$ generated by $N(e)$ and $e$. The quotient heap $C(e)=B / \overline{N(e)}$ is the coequalizer of 4.4 .2 .

3. If 4.4.2 is a diagram in $T$-mod, where $T$ is a truss, then $C(e)$ is its coequalizer in T-mod.

Proof. 1 That $N(e)$ is a sub-heap of $B$ follows by 1.1 .2 and the fact that $\varphi, \psi$ are morphisms of heaps. Let $f \in B$. The isomorphism between $N(e)$ and $N(f)$ is given by $\tau_{e}^{f}$ of 1.1 .4 .

2 Let us check that the canonical projection $\pi: B \longrightarrow C(e)=B / \overline{N(e)}$ coequalizes $\varphi$ and $\psi$. Since $e \in \overline{N(e)}$ and $[\varphi(a), \psi(a), e] \in \overline{N(e)}, \varphi(a) \sim_{\overline{N(e)}} \psi(a)$, and hence $\pi(\varphi(a))=\pi(\psi(a))$. Therefore, there is the required fork

$$
A \underset{\psi}{\stackrel{\varphi}{\longrightarrow}} \longrightarrow \stackrel{\pi}{\longrightarrow} C(e)
$$

Now, let us assume that there exists another pair $(h, H)$ such that $h: B \rightarrow H$ and $h \circ \varphi=h \circ \psi$. Observe that, for all $a \in A$,

$$
h([\varphi(a), \psi(a), e])=[h(\varphi(a)), h(\psi(a)), h(e)]=h(e),
$$

where the second equality follows from $h \circ \varphi=h \circ \psi$ and Mal'cev identity. Thus, $h(x)=h(e)$ for all $x \in \overline{N(e)}$ and so $\overline{N(e)} \subseteq \operatorname{ker}_{h(e)}(h)$. In view of Lemma 1.2.17, there is a unique heap homomorphism $f: C(e) \rightarrow H$ given by $f(\pi(b))=h(b)$ for all $b \in B$.

3 To prove that $C(e)$ is a coequalizer in the category of modules it is enough to prove that $\overline{N(e)}$ is an induced $T$-submodule. Since

$$
\begin{aligned}
t \cdot e[\varphi(a), \psi(a), e] & =[t \cdot[\varphi(a), \psi(a), e], t \cdot e, e]=[[t \cdot \varphi(a), t \cdot \psi(a), t \cdot e], t \cdot e, e] \\
& =[t \cdot \varphi(a), t \cdot \psi(a),[t \cdot e, t \cdot e, e]]=[t \cdot \varphi(a), t \cdot \psi(a), e] \\
& =[\varphi(t a), \psi(t a), e] \in \overline{N(e)}
\end{aligned}
$$

and $t \cdot{ }_{e} e=e$, it follows that $C(e)$ is a well-defined quotient module and the proof that $C(e)$ is the coequalizer is analogous to 2 . 


\section{Chapter 5}

\section{Extensions of trusses}

There is a one-sided connection between rings and trusses given by the functor

T : Ring $\rightarrow$ Trs. An analogous functor can be constructed from the category of braces to the category of trusses. In this chapter we investigate possibilities of assigning a ring, a brace or a unital truss to a truss. This chapter consists of two sections.

In Section 5.1, we introduce different methods to extend a truss: we consider a product with a unital module, and a coproduct with a terminal module. The first approach allows us to extend a brace to a new brace, or dually, extend a unital module over a brace to a brace. The new brace is not necessarily one that the module is over. The coproduct approach allows us to extend a truss to a ring or a unital truss. This depends on how we interpret a terminal module. If we assign it a role of an identity, we get a unital module. If we assign it a role of an absorber, we get a ring. This section is based on [19] and [32].

In Section 5.2, we further study connection between trusses and rings. A left adjoint to the functor $\mathrm{T}$ is constructed. The universal property given by the adjunction allows us to introduce three definitions of "smallness": locally small extensions, small extensions and minimal extensions. We conclude this section with a remark with a possible application of minimal extensions of trusses to essential extensions of rings. This section is based on [34]. 


\subsection{On extensions of a truss}

In this section we study various ways to extend trusses to rings, braces and unital trusses. We introduce two methods: one by taking a product of truss with a unital module over the truss, and second, by taking a coproduct with a terminal module over the truss.

\subsubsection{Extension by a module}

By the standard construction, given a ring $R$ and an $R$-bimodule $M$ one can define an extension of $R$ by $M$ as a ring with the Abelian group structure $R \oplus M$ and multiplication $(r, m)\left(r^{\prime}, m^{\prime}\right)=\left(r r^{\prime}, r m^{\prime}+m r^{\prime}\right)$. In this section we show that this construction can be extended to one-sided modules, but then the result is a truss rather than a ring.

Let us start with the following motivating observations.

Example 5.1.1. Let $G$ be an Abelian group. Then the ring of endomorphisms $\mathbf{A b}(G, G)$ acts on $G$ by evaluation, i.e. $\mathbf{A b}(G, G) \times G \longrightarrow G,(f, g) \mapsto f(g)$. One easily checks that the following binary operation on $\mathbf{A b}(G, G) \oplus G$

$$
(f, g)\left(f^{\prime}, g^{\prime}\right)=\left(f \circ f^{\prime}, g+f\left(g^{\prime}\right)\right), \quad \text { for all } f, f^{\prime} \in \mathbf{A b}(G, G), g, g^{\prime} \in G,
$$

is associative. However, the operation (5.1.1) does not distribute over the addition $\operatorname{Ab}(G, G) \oplus G$, since for all $f, f^{\prime}, f^{\prime \prime} \in \mathbf{A b}(G, G)$ and $g, g^{\prime}, g^{\prime \prime} \in G$, on one hand

$$
(f, g)\left(\left(f^{\prime}, g^{\prime}\right)+\left(f^{\prime \prime}, g^{\prime \prime}\right)\right)=\left(f \circ f^{\prime}+f \circ f^{\prime \prime}, g+f\left(g^{\prime}\right)+f\left(g^{\prime \prime}\right)\right)
$$

while on the other

$$
(f, g)\left(f^{\prime}, g^{\prime}\right)+(f, g)\left(f^{\prime \prime}, g^{\prime \prime}\right)=\left(f \circ f^{\prime}+f \circ f^{\prime \prime}, g+g+f\left(g^{\prime}\right)+f\left(g^{\prime \prime}\right)\right)
$$

Notwithstanding, it is easy to check that the operation 5.1.1 distributes over the ternary heap operation associated to the addition in $\mathbf{A b}(G, G) \oplus G$. In summary, the extension of the endomorphism ring of a group by this group is a truss. 
Now we place Example 5.1.1 in a more general framework of extensions of trusses by one-sided modules.

Theorem 5.1.2. Let $T$ be a truss and let $M$ be a left $T$-module. Then, for all $e \in M$, $T \times M$ is a truss with the Cartesian product heap structure and multiplication

$$
(t, m)\left(t^{\prime}, m^{\prime}\right)=\left(t t^{\prime},\left[m, t \cdot e, t \cdot m^{\prime}\right]\right)
$$

for all $t, t^{\prime} \in T$ and $m, m^{\prime} \in M$. We denote this truss by $T[M ; e]$ and call it an extension of $T$ by $M$.

Proof. That $T \times M$ with given operations is a truss can be checked by direct calculations. We start with the associative law. For all $t, t^{\prime}, t^{\prime \prime} \in T$ and $m, m^{\prime}, m^{\prime \prime} \in M$,

$$
\begin{aligned}
(t, m)\left(\left(t^{\prime}, m^{\prime}\right)\left(t^{\prime \prime}, m^{\prime \prime}\right)\right) & =(t, m)\left(t^{\prime} t^{\prime \prime},\left[m^{\prime}, t^{\prime} \cdot e, t^{\prime} \cdot m^{\prime \prime}\right]\right) \\
& =\left(t t^{\prime} t^{\prime \prime},\left[m, t \cdot e, t \cdot\left[m^{\prime}, t^{\prime} \cdot e, t^{\prime} \cdot m^{\prime \prime}\right]\right]\right) \\
& =\left(t t^{\prime} t^{\prime \prime},\left[\left[m, t \cdot e, t \cdot m^{\prime}\right], t t^{\prime} \cdot e, t t^{\prime} \cdot m^{\prime \prime}\right]\right) \\
& =\left(t t^{\prime},\left[m, t \cdot e, t \cdot m^{\prime}\right]\right)\left(t^{\prime \prime}, m^{\prime \prime}\right)=\left((t, m)\left(t^{\prime}, m^{\prime}\right)\right)\left(t^{\prime \prime}, m^{\prime \prime}\right),
\end{aligned}
$$

where the third equality follows by the distributive and associative laws for modules over trusses and by the associativity of the heap operation. To prove the left distributive law we compute, for all $t, t^{\prime}, t^{\prime \prime}, t^{\prime \prime \prime} \in T$ and $m, m^{\prime}, m^{\prime \prime}, m^{\prime \prime \prime} \in M$ :

$$
\begin{aligned}
(t, m)\left[\left(t^{\prime}, m^{\prime}\right),\right. & \left.\left(t^{\prime \prime}, m^{\prime \prime}\right),\left(t^{\prime \prime \prime}, m^{\prime \prime \prime}\right)\right]=(t, m)\left(\left[t^{\prime}, t^{\prime \prime}, t^{\prime \prime \prime}\right],\left[m^{\prime}, m^{\prime \prime}, m^{\prime \prime \prime}\right]\right) \\
& =\left(t\left[t^{\prime}, t^{\prime \prime}, t^{\prime \prime \prime}\right],\left[m, t \cdot e, t \cdot\left[m^{\prime}, m^{\prime \prime}, m^{\prime \prime \prime}\right]\right)\right. \\
& =\left(\left[t t^{\prime}, t t^{\prime \prime}, t t^{\prime \prime \prime}\right],\left[[m, m, m],[t \cdot e, t \cdot e, t \cdot e],\left[t \cdot m^{\prime}, t \cdot m^{\prime \prime}, t \cdot m^{\prime \prime \prime}\right]\right]\right) \\
& =\left(\left[t t^{\prime}, t t^{\prime \prime}, t t^{\prime \prime \prime}\right],\left[\left[m, t \cdot e, t \cdot m^{\prime}\right],\left[m, t \cdot e, t \cdot m^{\prime \prime}\right],\left[m, t \cdot e, t \cdot m^{\prime \prime \prime}\right]\right]\right) \\
& =\left[(t, m)\left(t^{\prime}, m^{\prime}\right),(t, m)\left(t^{\prime \prime}, m^{\prime \prime}\right),(t, m)\left(t^{\prime \prime \prime}, m^{\prime \prime \prime}\right)\right] .
\end{aligned}
$$

The third equality follows by the distributive laws for trusses and modules over trusses and by the Mal'cev identities (which imply that the heap operation is an idempotent operation). The rearrangement of brackets leading to the fourth equality is possible 
since $M$ is an Abelian heap. Similarly, for the right distributivity,

$$
\begin{aligned}
{\left[\left(t^{\prime}, m^{\prime}\right),\right.} & \left.\left(t^{\prime \prime}, m^{\prime \prime}\right),\left(t^{\prime \prime \prime}, m^{\prime \prime \prime}\right)\right](t, m)=\left(\left[t^{\prime}, t^{\prime \prime}, t^{\prime \prime \prime}\right],\left[m^{\prime}, m^{\prime \prime}, m^{\prime \prime \prime}\right]\right)(t, m) \\
& =\left(\left[t^{\prime} t, t^{\prime \prime} t, t^{\prime \prime \prime} t\right],\left[\left[m^{\prime}, m^{\prime \prime}, m^{\prime \prime \prime}\right],\left[t^{\prime}, t^{\prime \prime}, t^{\prime \prime \prime}\right] \cdot e,\left[t^{\prime}, t^{\prime \prime}, t^{\prime \prime \prime}\right] \cdot m\right]\right) \\
& =\left(\left[t^{\prime} t, t^{\prime \prime} t, t^{\prime \prime \prime} t\right],\left[\left[m^{\prime}, m^{\prime \prime}, m^{\prime \prime \prime}\right],\left[t^{\prime} \cdot e, t^{\prime \prime} \cdot e, t^{\prime \prime \prime} \cdot e\right],\left[t^{\prime} \cdot m, t^{\prime \prime} \cdot m, t^{\prime \prime \prime} \cdot m\right]\right]\right) \\
& =\left(\left[t^{\prime} t, t^{\prime \prime} t, t^{\prime \prime \prime} t\right],\left[\left[m^{\prime}, t^{\prime} \cdot e, t^{\prime} \cdot m\right],\left[m^{\prime \prime}, t^{\prime \prime} \cdot e, t^{\prime \prime} \cdot m\right],\left[m^{\prime \prime \prime}, t^{\prime \prime \prime} \cdot e, t^{\prime \prime \prime} \cdot m\right]\right]\right) \\
& =\left[\left(t^{\prime}, m^{\prime}\right)(t, m),\left(t^{\prime \prime}, m^{\prime \prime}\right)(t, m),\left(t^{\prime \prime \prime}, m^{\prime \prime \prime}\right)(t, m)\right] .
\end{aligned}
$$

Here, as in the preceding computation, the third equality is obtained by the distributive laws, while the fourth one follows from the fact that $M$ is an Abelian heap. Since the operation (5.1.2) is associative and distributes from both sides over the heap operation in $T \times M, T[M ; e]$ is a truss, as claimed.

A natural question that arises here is whether $T[M ; e]$ can be a truss associated with a ring.

Lemma 5.1.3. The truss $T[M ; e]$ is ring-type if and only if $M=\{e\}$ and $T=\mathrm{T}(R)$ for some ring $R$.

Proof. Let $T[M ; e]$ be a ring-type truss and $\left(t^{\prime}, m^{\prime}\right)$ be an absorber in $T[M ; e]$. Then, for all $m \in M$ and $t \in T$,

$$
\left(t^{\prime} t,\left[m^{\prime}, t^{\prime} \cdot e, t^{\prime} \cdot m\right]\right)=\left(t^{\prime}, m^{\prime}\right)(t, m)=\left(t^{\prime}, m^{\prime}\right)=(t, m)\left(t^{\prime}, m^{\prime}\right)=\left(t t^{\prime},\left[m, t \cdot e, t \cdot m^{\prime}\right]\right)
$$

which immediately implies that $t^{\prime}$ is the absorber in $T$. Therefore, $T=\mathrm{T}(R)$, where $R$ has the same multiplication as $T$ and the Abelian group structure obtained as the $t^{\prime}$-retract of $(T,[-,-,-])$.

Observe that $\left[m, t \cdot e, t \cdot m^{\prime}\right]=m^{\prime}$ implies $t \cdot m^{\prime}=\left[t \cdot e, m, m^{\prime}\right]$, so choosing $m=t \cdot e$ we obtain $t \cdot m^{\prime}=m^{\prime}$, for all $t \in T$, i.e. $m^{\prime}$ is an absorber in $M$. Hence $m^{\prime}=\left[t \cdot e, m, m^{\prime}\right]$, for all $m \in M$. In particular, for $m=e, t \cdot e=e$. Therefore, for all $m \in M$,

$$
\left[e, m, m^{\prime}\right]=m^{\prime}
$$

which implies that $m=e$. So if the truss $T[M ; e]$ is ring-type, then $M=\{e\}$. 
The converse implication follows by the simple observation that $\mathrm{T}(R) \cong \mathrm{T}(R)[\{e\} ; e]$.

Put differently, Lemma 5.1.3 asserts that the truss obtained by extension by a non-trivial module is never a truss associated to a ring.

We now list properties of an extension truss.

Theorem 5.1.4. Let $T$ be a truss, $M$ be a left $T$-module and let $e \in M$.

(1) For any $\bar{e} \in M, T[M ; e] \cong T[M ; \bar{e}]$.

(2) $M$ is a left $T[M ; e]$-module with the action, for all $m, m^{\prime} \in M$ and $t \in T$,

$$
(t, m) \cdot m^{\prime}=\left[m, t \cdot e, t \cdot m^{\prime}\right]
$$

In particular, $(t, m) \cdot e=m$.

(3) The induced actions of $T[M ; e]$ on $M$ coincide with the induced actions of $T$ on $M$, i.e. for all $\bar{e} \in M$

$$
(t, m) \triangleright_{\bar{e}} m^{\prime}=t \triangleright_{\bar{e}} m^{\prime}
$$

In particular, if $\bar{e}$ is an absorber in the $T$-module $M$, then $(t, m) \cdot \bar{e} m^{\prime}=t \cdot m^{\prime}$.

(4) For all $a \in T$, the sub-heap $M_{a}:=\{a\} \times M$ is a paragon in $T[M ; e]$. Furthermore,

$$
T[M ; e] / M_{a} \cong T
$$

$M_{a}$ is an ideal in $T[M ; e]$ if and only if $a$ is an absorber in $T$.

(5) The sub-heap $T_{e}:=T \times\{e\}$ is a sub-truss and a left paragon of $T[M ; e]$. Furthermore,

$$
T[M ; e] / T_{e} \cong M
$$

as left $T[M ; e]$-modules.

(6) The extension truss $T[M ; e]$ is unital if and only if $T$ is a unital truss and $M$ is a unital module. Furthermore, $U(T[M ; e])=U(T) \times M$. 
Proof. (1) Consider the heap automorphism:

$$
\Theta=1_{T} \times \tau_{e}^{\bar{e}}: T \times M \longrightarrow T \times M, \quad(t, m) \longmapsto(t,[m, e, \bar{e}]) .
$$

We will show that $\Theta$ is a truss isomorphism from $T[M ; e]$ to $T[M ; \bar{e}]$. Let us take any $t, t^{\prime} \in T$ and $m, m^{\prime} \in M$, and compute

$$
\begin{aligned}
\Theta(t, m) \Theta\left(t^{\prime}, m^{\prime}\right) & =(t,[m, e, \bar{e}])\left(t^{\prime},\left[m^{\prime}, e, \bar{e}\right]\right) \\
& =\left(t t^{\prime},\left[[m, e, \bar{e}], t \cdot \bar{e}, t \cdot\left[m^{\prime}, e, \bar{e}\right]\right]\right) \\
& =\left(t t^{\prime},\left[[m, e, \bar{e}], t \cdot \bar{e},\left[t \cdot m^{\prime}, t \cdot e, t \cdot \bar{e}\right]\right]\right) \\
& =\left(t t^{\prime},\left[\left[t \cdot m^{\prime}, t \cdot e, t \cdot \bar{e}\right], t \cdot \bar{e},[m, e, \bar{e}]\right]\right) \\
& =\left(t t^{\prime},\left[\left[t \cdot m^{\prime}, t \cdot e, m\right], e, \bar{e}\right]\right) \\
& =\left(t t^{\prime},\left[\left[m, t \cdot e, t \cdot m^{\prime}\right], e, \bar{e}\right]\right)=\Theta\left((t, m)\left(t^{\prime}, m^{\prime}\right)\right),
\end{aligned}
$$

where the third equality follows by the left distributive law for actions. The fourth and sixth equalities are consequences of the fact that $M$ is an Abelian heap. The key cancellation and rearrangement of brackets leading to the fifth equality result from the associative laws for and Mal'cev properties of heap operations. Thus $\Theta$ is the required isomorphism of trusses.

(2) The proof of the associative and distributive laws for $M$ as a $T[M ; e]$-module follow by the same chains of arguments as that in the proof of Theorem 5.1 .2 for the corresponding laws for the truss $T[M ; e]$, and thus are left to the reader. The property $(t, m) \cdot e=m$ follows immediately by the Mal'cev identity.

(3) For the first statement, observe that

$$
\begin{aligned}
(t, m) \triangleright_{\bar{e}} m^{\prime} & =\left[(t, m) \cdot m^{\prime},(t, m) \cdot \bar{e}, \bar{e}\right] \\
& =\left[\left[m, t \cdot e, t \cdot m^{\prime}\right],[m, t \cdot e, t \cdot \bar{e}], \bar{e}\right]=\left[t \cdot m^{\prime}, t \cdot \bar{e}, \bar{e}\right]=t \triangleright_{\bar{e}} m^{\prime},
\end{aligned}
$$

by the Mal'cev identities, associativity of the heap operation and by the fact that $M$ is an Abelian heap. The second statement follows immediately by the Mal'cev identity.

(4) For all $t \in T, m, m^{\prime}, m^{\prime \prime} \in M$,

$$
\begin{aligned}
{\left[(t, m)\left(a, m^{\prime}\right),(t, m)(a, e),(a, e)\right] } & =\left[\left(t a,\left[m, t \cdot e, t \cdot m^{\prime}\right]\right),(t a,[m, t \cdot e, t \cdot e]),(a, e)\right] \\
& =\left([t a, t a, a],\left[\left[m, t \cdot e, t \cdot m^{\prime}\right], m, e\right]\right) \\
& =\left(a,\left[t \cdot m^{\prime}, t \cdot e, e\right]\right) \in M_{a}
\end{aligned}
$$


by the Mal'cev identities and the associativity of heap operations. Hence $M_{a}$ is a left paragon. The right paragon property of $M_{a}$ is proven in a similar way.

Consider the following maps

$$
\begin{array}{ll}
\varphi: T \longrightarrow T[M ; e] / M_{a}, & t \longmapsto \overline{(t, e)} \\
\psi: T[M ; e] / M_{a} \longrightarrow T, & \overline{(t, m)} \longmapsto t .
\end{array}
$$

The map $\varphi$ is the quotient of the heap map $\tilde{\varphi}: T \longrightarrow T[M ; e]$, given by $t \longmapsto(t, e)$. Note that, for all $t, t^{\prime} \in T$,

$$
\tilde{\varphi}(t) \tilde{\varphi}\left(t^{\prime}\right)=(t, e)\left(t^{\prime}, e\right)=\left(t t^{\prime},[e, t \cdot e, t \cdot e]\right)=\left(t t^{\prime}, e\right)=\tilde{\varphi}\left(t t^{\prime}\right)
$$

i.e. $\tilde{\varphi}$ is a truss homomorphism, and thus so is $\varphi$.

We need to check whether the map $\psi$ is well-defined. By the definitions of $M_{a}$ and the sub-heap relation, $(t, m) \sim_{M_{a}}\left(t^{\prime}, m^{\prime}\right)$ if and only if there exist $m^{\prime \prime}, m^{\prime \prime \prime} \in M$ such that

$$
\left(a, m^{\prime \prime \prime}\right)=\left[(t, m),\left(t^{\prime}, m^{\prime}\right),\left(a, m^{\prime \prime}\right)\right]=\left(\left[t, t^{\prime}, a\right],\left[m, m^{\prime}, m^{\prime \prime}\right]\right) .
$$

Thus, in particular $a=\left[t, t^{\prime}, a\right]$ which implies $t^{\prime}=t$. Therefore, the element $t$ is fully determined by the class of $(t, m)$. This means that the function $\psi$ is well-defined. The second consequence of $(5.1 .3)$ is that the class of $(t, m)$ is fully determined by $t$, i.e. it does not depend on the choice of $m$. This implies that the composite function $\varphi \circ \psi$ is the identity. That the composite $\psi \circ \varphi$ is identity is obvious.

The fact that $M_{a}$ is an ideal in $T[M ; e]$ if and only if $a$ is an absorber in $T$ follows immediately from the analysis of the first entries in the products $(t, m)\left(a, m^{\prime}\right)$ and $(a, m)\left(t, m^{\prime}\right)$.

(5) It is obvious that $T_{e}$ is closed under the ternary heap operation. That $T_{e}$ is closed under the multiplication as well follows immediately by the Mal'cev identity. Let us take $\left(t^{\prime \prime}, m\right) \in T[M ; e]$ and $\left(t^{\prime}, e\right),(t, e) \in T_{e}$, and use the definition of the truss operations for $T[M ; e]$ and Mal'cev identities to compute:

$$
\begin{aligned}
{\left[\left(t^{\prime \prime}, m\right)\left(t^{\prime}, e\right),\left(t^{\prime \prime}, m\right)(t, e),(t, e)\right] } & =\left[\left(t^{\prime \prime} t^{\prime},\left[m, t^{\prime \prime} \cdot e, t^{\prime \prime} \cdot e\right]\right),\left(t^{\prime \prime} t,\left[m, t^{\prime \prime} \cdot e, t^{\prime \prime} \cdot e\right]\right),(t, e)\right] \\
& =\left(\left[t^{\prime \prime} t^{\prime}, t^{\prime \prime} t, t\right],[m, m, e]\right)=\left(\left[t^{\prime \prime} t^{\prime}, t^{\prime \prime} t, t\right], e\right) \in T_{e}
\end{aligned}
$$


Therefore, $T_{e}$ is a left paragon hence a left induced $T[M ; e]$-submodule of $T[M ; e]$. The left $T[M ; e]$-module isomorphism $T[M ; e] / T_{e} \longrightarrow M$ is constructed in the similar way to the isomorphism in part (4). In particular, one finds that, for all $t, t^{\prime} \in T$ and $m, m^{\prime} \in M$

(a) $(t, e) \sim_{T_{e}}\left(t^{\prime}, e\right)$ and

(b) if $(t, m) \sim_{T_{e}}\left(t^{\prime}, m^{\prime}\right)$, then $m=m^{\prime}$.

Hence we can fix $a \in T$ and consider the following functions:

$$
\begin{array}{ll}
\varphi: M \longrightarrow T[M ; e] / T_{e}, & m \longmapsto \overline{(a, m)}, \\
\psi: T[M ; e] / T_{e} \longrightarrow M, & \overline{(t, m)} \longmapsto m .
\end{array}
$$

The map $\varphi$ is the quotient of a heap homomorphism $m \longmapsto(a, m)$ hence a heap homomorphism. Furthermore, using observation (a) one can compute

$$
\begin{aligned}
(t, m) \cdot \varphi\left(m^{\prime}\right) & =\overline{(t, m)\left(a, m^{\prime}\right)}=\overline{\left(t a,\left[m, t \cdot e, t \cdot m^{\prime}\right]\right)} \\
& =\overline{\left(a,\left[m, t \cdot e, t \cdot m^{\prime}\right]\right)}=\overline{\left(a,(t, m) \cdot m^{\prime}\right)}=\varphi\left((t, m) \cdot m^{\prime}\right) .
\end{aligned}
$$

Hence $\varphi$ is a homomorphism of $T[M ; e]$-modules. That $\psi$ is well-defined follows by the observation (b). Again (a) implies that $\varphi \circ \psi=\mathrm{id}$ and the other inverse property is obvious.

(6) If $T$ has identity 1 , then $(1, e)$ is the identity for the extended truss $T[M ; e]$ by the Mal'cev properties and by the unitality of $M$. Conversely, if $(a, \bar{e})$ is the identity of $T[M ; e]$, then, for all $(t, m) \in T[M ; e]$,

$$
\begin{aligned}
(t, m) & =(a, \bar{e})(t, m)=(a t,[\bar{e}, a \cdot e, a \cdot m]), \\
(t, m) & =(t, m)(a, \bar{e})=(t a,[m, t \cdot e, t \cdot \bar{e}]) .
\end{aligned}
$$

Comparison of the first elements in each pair in equalities (5.1.4) yields that $T$ is unital with the identity $1=a$. Evaluation of (5.1.4a) at $m=e$ produces the equality $\bar{e}=e$, while its evaluation at $m=1 \cdot e$ gives $\bar{e}=1 \cdot e$, and hence $1 \cdot m=m$, for all $m \in M$, again by 5.1.4a). 
Let $u$ be a unit in $T$. Then, for all $m \in M$,

$$
\begin{aligned}
(u, m)\left(u^{-1},\left[e, u^{-1} \cdot m, u^{-1} \cdot e\right]\right) & =\left(u u^{-1},\left[m, u \cdot e, u \cdot\left[e, u^{-1} \cdot m, u^{-1} \cdot e\right]\right]\right) \\
& \left.=\left(1,\left[[m, u \cdot e, u \cdot e], u u^{-1} \cdot m, u u^{-1} \cdot e\right]\right)\right]=(1, e),
\end{aligned}
$$

by the distributive and associative laws for modules, axioms of heaps and unitality of $M$. In a similar way, by the axioms of a heap

$$
\left(u^{-1},\left[e, u^{-1} \cdot m, u^{-1} \cdot e\right]\right)(u, m)=\left(u^{-1} u,\left[\left[e, u^{-1} \cdot m, u^{-1} \cdot e\right], u^{-1} \cdot e, u^{-1} \cdot m\right]\right)=(1, e) .
$$

Hence, if $u$ is a unit in $T,(u, m)$ is a unit in $T[M ; e]$, for all $m \in M$. This proves the inclusion $U(T) \times M \subseteq U(T[M ; e])$. The converse inclusion follows immediately from the definition of the product in $T[M ; e]$.

Remark 5.1.5. Assertions (4) and (5) of Theorem 5.1.4 yield the following sequence, for all $a \in T$,

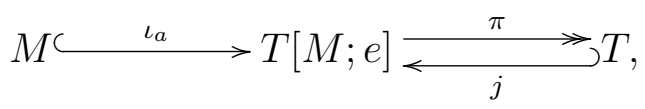

where $\iota_{a}: m \longmapsto(a, m), j: t \longmapsto(t, e)$ and $\pi:(t, m) \longmapsto t$. This sequence is a split-exact sequence of trusses in the following sense. The map $\pi$ is a split epimorphism of trusses ( $j$ is the splitting monomorphism) and the relation induced by the image of $\iota_{a}$ is the kernel relation for $\pi$. In summary, Theorem 5.1 .2 describes a split extension of trusses.

The assertion (6) of Theorem 5.1.4 implies the following

Corollary 5.1.6. An extension truss $T[M ; e]$ is the truss associated to a two-sided brace if and only if $T$ is associated to a two-sided brace and $M$ is a unital T-module.

Proof. By Theorem 5.1.4 (6) $T[M ; e]$ is brace-type (unital) if and only if $T$ is bracetype. Furthermore, $U(T[M ; e])=T[M ; e]$ if, and only if $U(T)=T$, i.e. $T[M ; e]$ is a multiplicative group (brace) if and only if $T$ is as well.

It might be instructive to contrast Lemma 5.1.3 with Corollary 5.1.6. While only trivial truss extension of a ring results in a ring (alas an extension in name only), an extension of a two-sided brace by any unital module over the associated truss is a two-sided brace. 
Example 5.1.7. (1) Let $B$ be a two-sided brace. Then $\mathrm{T}(B)$ is a left module over itself by multiplication, hence one can consider the extension truss $\mathrm{T}(B)[\mathrm{T}(B) ; 1]$. As a heap,

$$
\mathrm{T}(B)[\mathrm{T}(B) ; 1]=\mathrm{T}(B) \times \mathrm{T}(B) .
$$

The multiplication comes out as, for all $b_{1}, b_{2}, b_{1}^{\prime}, b_{2}^{\prime} \in B$,

$$
\left(b_{1}, b_{2}\right)\left(b_{1}^{\prime}, b_{2}^{\prime}\right)=\left(b_{1} b_{1}^{\prime}, b_{2}-b_{1}+b_{1} b_{2}^{\prime}\right)
$$

$\mathrm{T}(B)[\mathrm{T}(B) ; 1]$ is the truss associated to the two-sided brace with additive structure given by $B \oplus B$ and multiplication given by the formula 5.1.5. Note that, even if the brace $B$ is Abelian (i.e. the truss $\mathrm{T}(B)$ is commutative), the extended brace need not be so.

For an explicit example we may consider the brace obtained as the 0-retract of the truss $\mathbb{Z}^{(2)} / 2^{k+1} \mathbb{Z}$ in Proposition 2.3.9. The multiplication in $\mathbb{Z}^{(2)} / 2^{k+1} \mathbb{Z}\left[\mathbb{Z}^{(2)} / 2^{k+1} \mathbb{Z} ; 0\right]$ is given by

$$
(m, s)(n, t)=\left(2 m n+m+n \bmod 2^{k+1}, 2 m t+s+t \bmod 2^{k+1}\right) .
$$

In particular, the multiplicative group of $\mathbb{Z}^{(2)} / 4 \mathbb{Z}\left[\mathbb{Z}^{(2)} / 4 \mathbb{Z} ; 0\right]$ is generated by elements

$$
a=(0,1), \quad x=(1,0), \quad y=(2,0),
$$

which satisfy the following relations

$$
a^{4}=x^{2}=y^{2}=(0,0), \quad x a x=a^{3}, \quad x y=y x, \quad a y=y a,
$$

and hence it is isomorphic to the direct product of the dihedral group $D_{8}$ and the cyclic group $C_{2}$. The additive structure of the two-sided brace associated to $\left.\mathbb{Z}^{(2)} / 4 \mathbb{Z}^{2} \mathbb{Z}^{(2)} / 4 \mathbb{Z} ; 0\right]$ is that of $C_{4} \oplus C_{4}$.

(2) Since the socle, $\operatorname{Soc}(B)$, is an ideal in a two-sided brace $B$, it is a left (induced) $\mathrm{T}(B)$-module. The multiplication on $\mathrm{T}(B)[\operatorname{Soc}(B) ; 1]=\mathrm{T}(B) \times \operatorname{Soc}(B)$ derived from 5.1.5 reduces to

$$
(b, a)\left(b^{\prime}, a^{\prime}\right)=\left(b b^{\prime}, a-b+b a\right)=\left(b b^{\prime}, a+b a b^{-1}\right) .
$$


Remark 5.1.8. In view of Theorem 5.1.4, since $M$ is a module over the extended truss $T[M ; e]$, the extension procedure could be iterated. This, however, rather than producing genuinely new examples boils down to the truss extension by the $T$-module obtained as the product of $T$-modules.

We conclude this part with the following

Remark 5.1.9. By analysing the proofs of main statements of this section, that is Theorem 5.1.2 and Theorem 5.1.4, one can easily convince oneself that the assertions hold for left trusses and their left modules (and also for right trusses if left modules are replaced by right ones). Since for a left truss $T$ no right distributivity is assumed, one does not assume that the action of $T$ on a left module $M$ right distributes over the heap operation on $T$ (otherwise $T$ would not be its own module). But this right distributivity is not needed neither for the associativity of the product in $(5.1 .2)$ in $T[M ; e]$ nor for its left distributivity. Thus, if $T$ is a left truss and $M$ is a left $T$-module, then $T[M ; e]$ with Cartesian product heap structure and with multiplication (5.1.2) is a left truss. Main assertions of Theorem 5.1.2 stand if the words "truss" or "paragon" are qualified by the adjective "left". Most importantly, the one-sided version of Corollary 5.1.6, with no changes in the formula for the multiplicative group structure, equips one with the procedure of obtaining left braces from left braces.

\subsubsection{Coproduct extensions}

In general it is not known if a coproduct $A \boxplus B$ of $T$-modules $A, B$ can be endowed with a non-trivial truss multiplication, i.e. different than $m^{\prime} \cdot m=m, m^{\prime} \cdot m=m^{\prime}$ or $m^{\prime} \cdot m=e$, for all $m, m^{\prime} \in A \boxplus B$ and a fixed $e \in A \boxplus B$. In this part we introduce two ways of endowing a $T$-module coproduct $T \boxplus\{*\}$ with a structure of a truss. The first one is a unital truss, while the second one is a truss associated with a ring.

Proposition 5.1.10. Let $T$ be a truss and let $Z$ be the truss on the singleton set $\{0\}$. Then $T \boxplus Z$ with multiplication · given by

$$
0 \cdot t=t \cdot 0=0 \text { and } t \cdot t^{\prime}=t t^{\prime}
$$


where $t, t^{\prime} \in T$ and $t t^{\prime}$ is multiplication in truss $T$, is a ring-type truss, which we term the ring extension of $T$ and denote by $T_{0}$.

Proof. First note that if a binary operation defined on the heap $H$ generated by a set $X$ is associative on elements of $X$ and distributes over the heap operation, then it is associative on the whole of $H$. The operation 5.1.6 is associative on $T \sqcup\{0\}$ and hence it is associative on all the generators of the heap $T \boxplus Z$. We need to show that this operation as defined in (5.1.6) can be extended to the whole of $T \boxplus Z$ as a distributive operation. To this end, for all $s \in T \boxplus Z$ consider two functions extending multiplication (5.1.6) to elements of $T \boxplus Z$ term-by-term, i.e.

$$
\begin{aligned}
& \lambda_{T}^{s}: T \longrightarrow T \boxplus Z \\
& t \longmapsto t \cdot s:=\left\{\begin{aligned}
& {\left[t \cdot s_{1}, t \cdot 0, \ldots, t \cdot 0, t \cdot s_{n}\right] } \\
&=\left[t s_{1}, 0, \ldots, 0, t s_{n}\right], \\
& {\left[t \cdot 0, t \cdot s_{1}, \ldots, t \cdot s_{n}, t \cdot 0\right] } \\
&=\left[0, t s_{1}, \ldots, t s_{n}, 0\right], \text { if } s=\left[s_{1}, 0, s_{2}, \ldots, 0, s_{n}\right], \\
& {\left[t \cdot s_{1}, t \cdot s_{2}, t \cdot 0\right]=\left[t s_{1}, t s_{2}, 0\right], } \text { if } s=\left[0, s_{1}, 0, \ldots, s_{n}, 0\right],
\end{aligned}\right.
\end{aligned}
$$

where $s_{i} \in T$, and

$$
\lambda_{Z}^{s}: Z \longrightarrow T \boxplus Z, \quad 0 \mapsto 0 \cdot s=0
$$

The latter of these functions is a well-defined homomorphism of heaps, for all $s \in T \boxplus Z$. To see that the former is so as well, we first establish that its definition is independent on the presentation of $s$. If

$$
\left[s_{1}, 0, \ldots, 0, s_{n}\right]=\left[s_{1}^{\prime}, 0, \ldots, 0, s_{n}^{\prime}\right]
$$

then the Mal'cev identities imply that

$$
s_{1}^{\prime}=\left[s_{1}, 0, \ldots, 0, s_{n}, s_{n}^{\prime}, 0, \ldots, 0, s_{2}^{\prime}, 0\right]
$$

Using the fact that $T \boxplus Z$ is an Abelian heap and Mal'cev identities again, all the 0 can be eliminated and one finds that

$$
s_{1}^{\prime}=\left[s_{1}, s_{2}^{\prime}, s_{2}, s_{3}^{\prime} \ldots, s_{n-1}, s_{n}^{\prime}, s_{n}\right]
$$


Therefore,

$$
\begin{aligned}
{\left[t s_{1}^{\prime}, 0, \ldots, 0, t s_{n}^{\prime}\right] } & =\left[t\left[s_{1}, s_{2}^{\prime}, s_{2}, s_{3}^{\prime} \ldots, s_{n-1}, s_{n}^{\prime}, s_{n}\right], 0, t s_{2}^{\prime}, \ldots, 0, t s_{n}^{\prime}\right] \\
& =\left[t s_{1}, t s_{2}^{\prime}, t s_{2}, t s_{3}^{\prime} \ldots, t s_{n-1}, t s_{n}^{\prime}, t s_{n}, 0, \ldots, 0, t s_{n}^{\prime}\right] \\
& =\left[t s_{1}, 0, \ldots, 0, t s_{n}\right]
\end{aligned}
$$

by the distributive law in $T$, the Mal'cev identities and the fact that $T$ is an Abelian heap. In the second case one notices that $\left[0, s_{1}, \ldots, s_{n}, 0\right]=\left[0, s_{1}^{\prime}, \ldots, s_{n}^{\prime}, 0\right]$ if and only if $\left[s_{1}, 0, \ldots, 0, s_{n}\right]=\left[s_{1}^{\prime}, 0, \ldots, 0, s_{n}^{\prime}\right]$ and thus the same arguments apply. In the third case, if $\left[s_{1}, s_{2}, 0\right]=\left[s_{1}^{\prime}, s_{2}^{\prime}, 0\right]$, then $s_{1}^{\prime}=\left[s_{1}, s_{2}, s_{2}^{\prime}\right]$ and again the distributive law and the Abelian heap properties imply the independence of the definition of $\lambda_{T}^{s}$ on the representation of $s$. Thus $\lambda_{T}^{s}$ is a well-defined function that is a heap morphism by the distributive law in $T$. The universal property of coproducts provides us with the unique fillers (in the category of heaps) in the following diagrams that can be considered for all $s \in T \boxplus Z:$

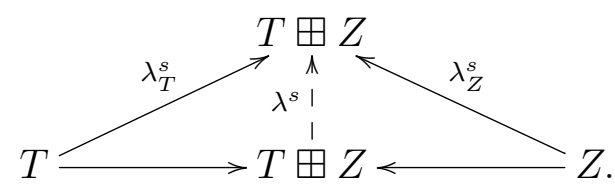

In this way the map

$$
\mu:(T \boxplus Z) \times(T \boxplus Z) \longrightarrow T \boxplus Z, \quad\left(s^{\prime}, s\right) \longmapsto \lambda^{s}\left(s^{\prime}\right),
$$

which extends the multiplication (5.1.7) to the whole of $T \boxplus Z$ has been constructed. This map is a heap homomorphism in both arguments (in the first argument by the universal construction described above, in the second one by the definition of $\lambda_{T}^{s}$ and $\lambda_{Z}^{s}$ ), that is it distributes over the heap operation in $T \boxplus Z$. This completes the proof.

Proposition 5.1.11. Let $T$ be a truss and $I$ be the truss on a singleton set $\{1\}$. Then $T \boxplus I$ with multiplication $\cdot$ given by

$$
1 \cdot 1=1,1 \cdot t=t \cdot 1=t \text { and } t \cdot t^{\prime}=t t^{\prime}
$$


where $t, t^{\prime} \in T$ and $t t^{\prime}$ is multiplication in $T$ is a unital truss, which we term the unital extension of $T$ and denote by $T_{u}$.

Proof. The proof is analogous to that of Proposition 5.1.10. We only note in passing that the maps $\lambda_{T}^{s}: T \rightarrow T \subset T \boxplus I, t \mapsto t s$, and $\lambda_{I}^{s}: I \rightarrow T \boxplus I, 1 \mapsto 1 \cdot s$, are well-defined since $\lambda_{I}^{s}$ is a constant map and $\lambda_{T}^{s}$ is the heap homomorphism on the direct sum of its modules (see [19, Proposition 3.12] for the direct proof).

Note that if $T$ is a ring-type truss with absorber 0 , then its unital extension $T_{u}$ remains to be a ring-type truss with (the same) absorber 0 .

The construction in Proposition 5.1.11 may be followed by that of Proposition 5.1.10 thus extending any truss $T$ to the unital ring-type truss $T \boxplus\{1\} \boxplus\{0\}$ (or a unital ring with the retract of the heap $T \boxplus\{1\}$ by 0 as the additive group). Note that any ring extension of a non-empty truss is an infinite ring, so while any ring can be interpreted as a truss, only some (and necessarily infinite at that) rings can be obtained as extensions of trusses. In particular one easily finds that $\mathrm{G}(\{1\} \boxplus\{0\} ; 0)$ together with the multiplication of the ring extension of $\{1\}$ is equal to the ring of integers. Presently, we describe other examples of unital and ring extensions of trusses.

Example 5.1.12. Let us consider the ring $\mathbb{Z}_{2}=\left\{i_{0}, i_{1}\right\}$, where $i_{0}$ is the zero and $i_{1}$ is the identity, and the associated truss $\mathrm{T}\left(\mathbb{Z}_{2}\right)$. In view of the isomorphism $\varphi$ in the proof of Proposition 1.4.4, the extension of $\mathrm{T}\left(\mathbb{Z}_{2}\right)$ by an absorber is

$$
\mathrm{G}\left(\mathrm{T}\left(\mathbb{Z}_{2}\right) \boxplus\{0\} ; 0\right):=\left\{\sigma u+k i_{0} \mid k \in \mathbb{Z}, \sigma \in \mathbb{Z}_{2}\right\}
$$

where $u=\left[i_{1}, i_{0}, 0\right],-+-=[-, 0,-]$ and the appearance of $\sigma$ implies the presence or absence of $u$. The formulae for addition and multiplication come out as:

$$
\begin{aligned}
\left(\sigma u+k i_{0}\right)+\left(\sigma^{\prime} u+k^{\prime} i_{0}\right) & =\left(\sigma+{ }_{(\bmod 2)} \sigma^{\prime}\right) u+\left(k+k^{\prime}\right) i_{0}, \\
\left(\sigma u+k i_{0}\right) \cdot\left(\sigma^{\prime} u+k^{\prime} i_{0}\right) & =\sigma \sigma^{\prime} u+k k^{\prime} i_{0} .
\end{aligned}
$$

Since $\mathrm{T}\left(\mathbb{Z}_{2}\right)$ is a truss with identity, so is its extension $\mathrm{T}\left(\mathbb{Z}_{2}\right)_{0}$; the identity is $i_{1}=u+i_{0}$.

Example 5.1.13. Let us consider the truss on the heap associated with $\mathbb{Z}$, whose multiplication is given by a constant $c$, i.e. $m n=c$ for all $m, n \in \mathbb{Z}$. We denote this 
truss as $\mathbb{Z}^{c}$ and describe the ring extension of $\mathbb{Z}^{c}$. To distinguish elements of $\mathbb{Z}$ from the integer multiplicities, we will use the symbols $i_{m}, m \in \mathbb{Z}$ for elements of $\mathbb{Z}^{c}$. In other words,

$$
\mathbb{Z}^{c}=\left\{i_{m} \mid m \in \mathbb{Z}\right\}, \quad\left[i_{k}, i_{l}, i_{m}\right]=i_{k-l+m}, \quad i_{m} i_{n}=i_{c} .
$$

By Proposition 1.4.4, the heap underlying $\mathbb{Z}_{0}^{c}=\{0\} \boxplus \mathbb{Z}^{c}$ is isomorphic to $H(\mathbb{Z} \oplus \mathbb{Z})$. Following Proposition 1.4.1 we choose 0 and $i_{c} \in \mathbb{Z}^{c}$ as special elements $e_{A}$ and $e_{B}$, respectively, and look at the retract $G\left(\{0\} \boxplus \mathbb{Z}^{c} ; 0\right)$ as the Abelian group underlying the ring $\mathbb{Z}_{0}^{c}$. In view of the isomorphism $\varphi$ in the proof of Proposition 1.4.4.

$$
\mathrm{G}\left(\{0\} \boxplus \mathbb{Z}^{c} ; 0\right)=\left\{\sigma i_{n}+k i_{c} \mid n \in \mathbb{Z} \backslash\{c\}, k \in \mathbb{Z}, \sigma \in \mathbb{Z}_{2}\right\}
$$

where $-+-=[-, 0,-]$. The appearance of $\sigma$ simply indicates either the absence or presence of $i_{n}$. The formulae for addition and multiplication in the ring $\left(\mathrm{G}\left(\{0\} \boxplus \mathbb{Z}^{c} ; 0\right), \cdot\right)$ come out as:

$$
\begin{gathered}
\left(\sigma i_{n}+k i_{c}\right)+\left(\sigma^{\prime} i_{n^{\prime}}+k^{\prime} i_{c}\right)=\sigma \sigma^{\prime}\left(i_{n-c+n^{\prime}}+i_{c}\right)+\left(1-\sigma^{\prime}\right) \sigma i_{n} \\
+(1-\sigma) \sigma^{\prime} i_{n^{\prime}}+\left(k+k^{\prime}\right) i_{c} \\
\left(\sigma i_{n}+k i_{c}\right) \cdot\left(\sigma^{\prime} i_{n^{\prime}}+k^{\prime} i_{c}\right)=\left(\sigma \sigma^{\prime}+\sigma k^{\prime}+\sigma^{\prime} k+k k^{\prime}\right) i_{c} .
\end{gathered}
$$

The ring extension of $\mathbb{Z}^{c}$ can be extended further to make it into a unital truss, $\mathbb{Z}_{0, u}^{c}=\{0\} \boxplus \mathbb{Z}^{c} \boxplus\{1\}$ as in Proposition 5.1.11. The corresponding retract is

$$
\mathrm{G}\left(\mathbb{Z}_{0, u}^{c} ; 0\right)=\left\{\sigma i_{n}+k i_{c}+l 1 \mid n \in \mathbb{Z} \backslash\{c\}, k, l \in \mathbb{Z}, \sigma \in \mathbb{Z}_{2}\right\}
$$

The binary operations are as follows

$$
\begin{aligned}
\left(\sigma i_{n}+k i_{c}+l 1\right)+\left(\sigma^{\prime} i_{n^{\prime}}+k^{\prime} i_{c}+l^{\prime} 1\right) & =\sigma \sigma^{\prime}\left(i_{n-c+n^{\prime}}+i_{c}\right)+\left(1-\sigma^{\prime}\right) \sigma i_{n} \\
& +(1-\sigma) \sigma^{\prime} i_{n^{\prime}}+(k+k)^{\prime} i_{c}+\left(l+l^{\prime}\right) 1 \\
\left(\sigma i_{n}+k i_{c}+l 1\right) \cdot\left(\sigma^{\prime} i_{n^{\prime}}+k^{\prime} i_{c}+l^{\prime} 1\right) & =\left(\sigma \sigma^{\prime}+\sigma k^{\prime}+\sigma^{\prime} k+k k^{\prime}+k l^{\prime}+l k^{\prime}\right) i_{c} \\
& +\sigma l^{\prime} i_{n}+\sigma^{\prime} l i_{n^{\prime}}+l l^{\prime} 1 .
\end{aligned}
$$

The retract $\mathrm{G}\left(\mathbb{Z}_{0, u}^{c} ; 0\right)$ with multiplication · is a unital ring.

Example 5.1.14. Let us consider the cyclic group $C_{2}=\{a, b\}$, where $a$ is the neutral element, with multiplication given by addition i.e. $a \cdot b=a+b=b$, etc. One can observe 
that $C_{2}$ with such operations is a brace, and so there is the associated truss, which we denote by $\mathrm{T}\left(C_{2}\right)$. This can be extended to $\mathrm{T}\left(C_{2}\right)_{0}=\{0\} \boxplus \mathrm{T}\left(C_{2}\right)$ as in Proposition 5.1.10. We choose 0 and $a$ as distinguished elements and, as in the preceding example, we study the ring structure on the retract $\mathrm{G}\left(\mathrm{T}\left(C_{2}\right)_{0} ; 0\right)$. Note that $[b, a, b]=a$ in $\mathrm{T}\left(C_{2}\right)$ yields the following relation in $\mathrm{G}\left(\mathrm{T}\left(C_{2}\right)_{0} ; 0\right)$,

$$
b+b=[b, 0, b]=[b, 0, b, a, a]=[[b, a, b], 0, a]=[a, 0, a]=a+a .
$$

Taking this into account $\mathrm{w}$ set $t=[b, a, 0] \in \mathrm{T}\left(C_{2}\right)_{0}$, and find that

$$
\mathrm{G}\left(\mathrm{T}\left(C_{2}\right)_{0} ; 0\right)=\left\{\sigma t+n a \mid \sigma \in \mathbb{Z}_{2}, n \in \mathbb{Z}\right\}
$$

The addition and multiplication in the ring $\mathrm{G}\left(\mathrm{T}\left(C_{2}\right)_{0} ; 0\right)$ come out as follows:

$$
\begin{aligned}
(\sigma t+n a)+\left(\sigma^{\prime} t+n^{\prime} a\right) & =\left(\sigma+(\bmod 2) \sigma^{\prime}\right) t+\left(n+n^{\prime}\right) a \\
(\sigma t+n a) \cdot\left(\sigma^{\prime} t+n^{\prime} a\right) & =\frac{1-(-1)^{\sigma^{\prime} n+\sigma n^{\prime}}}{2} t+n n^{\prime} a .
\end{aligned}
$$

We note in passing that since $a$ is the multiplicative identity of the brace $C_{2}$, the ring $\mathrm{G}\left(\mathrm{T}\left(C_{2}\right)_{0} ; 0\right)$ also has identity $a$.

A few comments appear to be in order now. Examples 5.1.12 \& 5.1.13 illustrate the fact that if a truss $T$ had an absorber, making the ring extension $T_{0}$ does not increase the number of absorbers (this would contradict the uniqueness of absorbers), but replaces the existing absorber by a new one. The truss $\mathbb{Z}^{c}$ has absorber $i_{c}$ which ceases to be an absorber in $\mathbb{Z}_{0}^{c}$ as $i_{c}\left(\sigma i_{n}+k i_{c}\right)=(\sigma+k) i_{c}$. Similar comment can be made about the unital extension: if a unital truss $T$, with identity $e$, is extended to $T_{u}$, then $u$ ceases to be the identity in $T_{u}$, as $1 e=e 1=e$ by the definition of the multiplication in $T_{u}$. One can also notice that the unital extension of the truss generated by a brace is no longer a truss generated by a brace (the fact that the ring extension is not a truss associated to a brace is obvious, since 0 is never an invertible element of a non-trivial ring). The easiest example is adding identity to the truss $\{*\}$ associated to the trivial brace $\{0\}$; $\{*\}_{u}$ is a ring-type truss which as a ring can be identified with $\mathbb{Z}$. Conceptually this can be understood by observing that the results of multiplication of any element from 
the truss associated with a brace $B$ and an element from the unital extension that does not belong to $B$ is an element of $B$ so there are no inverses in $\mathrm{T}(B)_{u}$ to elements in $B$.

Finally, let us observe that the ring obtained from the unital extension of the truss $\mathrm{T}(R)$ associated to a ring $R$ is the same as the Dorroh extension of $R$ [35].

Indeed, we know that $\mathrm{T}(R)_{u}=\mathrm{T}(R) \boxplus\{1\} \cong \mathrm{H}(R \oplus \mathbb{Z})$, we can choose $0 \in R$ and 1 to be distinguished elements and study the ring structure on the retract

$$
\mathrm{G}\left(\mathrm{T}(R)_{u} ; 0\right)=\{r+n \mid r \in R, n \in \mathbb{Z}\}=R \oplus \mathbb{Z} .
$$

Since 0 is an absorber in $\mathrm{T}(R)$ it remains an absorber in the unital truss $\mathrm{T}(R)_{u}$ and we can write down the multiplication formula as

$$
\begin{aligned}
(r+n) \cdot\left(r^{\prime}+n^{\prime}\right) & =[r, 0, n] \cdot\left[r^{\prime}, 0, n^{\prime}\right]=\left[r\left[r^{\prime}, 0, n^{\prime}\right], 0\left[r^{\prime}, 0, n^{\prime}\right], n\left[r^{\prime}, 0, n^{\prime}\right]\right] \\
& =\left[r r^{\prime}, 0, r n^{\prime}, 0, n r^{\prime}, 0, n n^{\prime}\right]=r r^{\prime}+r n^{\prime}+n r^{\prime}+n n^{\prime} .
\end{aligned}
$$

This is precisely the multiplication rule for the Dorroh extension of the ring $R$.

\subsection{Universal and minimal extensions to rings}

The preceding section introduces the method of extending a truss into a ring. We consider a coproduct of a truss with a singleton module over the truss, and define a particular multiplication on the coproduct, see Proposition 5.1.10. This multiplication coincides with the truss multiplication on elements of the truss and assign a role of an absorber to the singleton. In this section, we further investigate this extension. This section is based on Part 3 of [34].

\subsubsection{Extending to a ring}

Let us denote by $\mathrm{R}_{0}(T)$ a ring acquired by taking a zero retract of the truss extension $T_{0}$ of a truss $T$. The extension $T_{0}$ is a heap $T \boxplus\{0\}$, where $\{0\}$ is a unique $T$-module, together with the multiplication. The multiplication on the heap is given on generators $T \sqcup\{0\}$ by $t \cdot t^{\prime}=t t^{\prime}$ and $t 0=0 t=0$, for all $t, t^{\prime} \in T$. Obviously, $\mathrm{T}\left(\mathrm{R}_{0}(T)\right)=T_{0}$. A homomorphism of rings $\varphi: R \longrightarrow R^{\prime}$ is also a homomorphism of corresponding trusses 
$\mathrm{T}(\varphi): \mathrm{T}(R) \longrightarrow \mathrm{T}\left(R^{\prime}\right)$. Whenever we write a composition of a truss homomorphism $\psi: T \longrightarrow \mathrm{T}(R)$ with $\varphi: R \longrightarrow R^{\prime}, \varphi \circ \psi$ we think of $\mathrm{T}(\varphi) \circ \psi$.

Lemma 5.2.1. Let $T$ be a truss. An extension $\mathrm{R}_{0}(T)$ has the following universal property. For any ring $R$ and a homomorphism of trusses $\varphi: T \longrightarrow \mathrm{T}(R)$ there exists a unique ring homomorphism $\widehat{\varphi}: \mathrm{R}_{0}(T) \longrightarrow R$ rendering commutative the following diagram

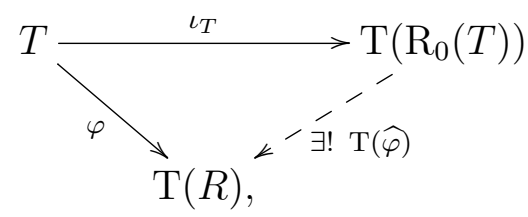

where $\iota_{T}: T \longrightarrow \mathrm{T}\left(\mathrm{R}_{0}(T)\right)$ is given by $t \longmapsto t$.

Proof. Let us consider the following commutative diagram of morphisms of trusses:

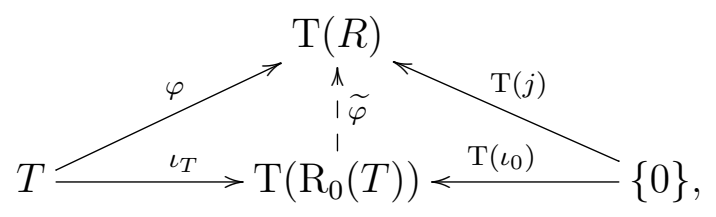

where $j$ and $\iota_{0}$ are unique ring homomorphisms from the zero object $\{0\}$ in the category of rings. The existence of the unique truss morphism $\widetilde{\varphi}: \mathrm{T}\left(\mathrm{R}_{0}(T)\right)=T \boxplus\{0\} \longrightarrow \mathrm{T}(R)$ follows by the universal property of the coproduct. Since

$$
\widetilde{\varphi} \circ \mathrm{T}\left(\iota_{0}\right)(0)=\widetilde{\varphi}(0)=\mathrm{T}(j)(0)=0_{R},
$$

the function $\widetilde{\varphi}=\mathrm{T}(\widehat{\varphi})$ for some (unique) ring homomorphism $\widehat{\varphi}: \mathrm{R}_{0}(T) \longrightarrow R$.

Corollary 5.2.2. A pair $\left(\mathrm{R}_{0}(T), \iota_{T}\right)$ is a universal arrow, see [24, Section III.1].

The preceding corollary already implies that $\mathrm{R}_{0}$ is a left adjoint to the functor $T$. Nevertheless, we present the full proof.

Lemma 5.2.3. Let $T$ be a truss. The truss homomorphism $\iota_{T}: T \longrightarrow T_{0}$ has the following cancellation property. For all truss homomorphisms $\varphi, \psi: \mathrm{T}_{0} \longrightarrow U$ such that $\varphi(0)=\psi(0)$,

$$
\varphi \circ \iota_{T}=\psi \circ \iota_{T} \text { implies } \varphi=\psi \text {. }
$$


In particular, if $U=\mathrm{T}(R)$ for a ring $R$, then for all ring homomorphisms $f, g: \mathrm{R}_{0}(T) \longrightarrow R$,

$$
\mathrm{T}(f) \circ \iota_{T}=\mathrm{T}(g) \circ \iota_{T} \text { implies } f=g .
$$

Proof. Let us consider a truss homomorphism $f: T_{0} \rightarrow S$. One can easily observe that by the uniqueness of the coproduct map $f=\left(f \circ \iota_{T}\right) \boxplus\left(f \circ \iota_{0}\right)$. Thus, because $f\left(\iota_{0}(0)\right)=g\left(\iota_{0}(0)\right)$ and $f \circ \iota_{T}=g \circ \iota_{T}$, we get

$$
f=\left(f \circ \iota_{T}\right) \boxplus\left(f \circ \iota_{0}\right)=\left(g \circ \iota_{T}\right) \boxplus\left(g \circ \iota_{0}\right)=g .
$$

Therefore, $f(0)=g(0)$ and $\left(f \circ \iota_{T}\right)=\left(g \circ \iota_{T}\right)$ implies $f=g$.

The universal property of the ring $\mathrm{R}_{0}(T)$ described in Lemma 5.2.1 gives rise to a functor $\mathrm{R}_{0}(-)$ : Trs $\longrightarrow$ Ring between categories of trusses and rings. The functor is given for all trusses $T$ by $T \longmapsto \mathrm{R}_{0}(T)$, and for all morphisms $\varphi \in \operatorname{Hom}_{\operatorname{Trs}}(T, U)$ by $\varphi \longmapsto \mathrm{R}_{0}(\varphi):=\widehat{\iota_{U} \circ \varphi}$, where ${ }^{\wedge}$ denotes the ring homomorphism induced from a truss homomorphism via the diagram in Lemma 5.2.1. Observe that by Lemma 5.2.1 for all $\varphi \in \operatorname{Hom}_{\text {Trs }}(T, U)$ and $\psi \in \operatorname{Hom}_{\text {Trs }}(U, V)$,

$$
\begin{aligned}
\mathrm{T}\left(\mathrm{R}_{0}(\psi) \circ \mathrm{R}_{0}(\varphi)\right) \circ \iota_{T} & =\mathrm{T}\left(\widehat{\iota_{V} \circ \psi}\right) \circ \mathrm{T}\left(\widehat{\iota_{U} \circ \varphi}\right) \circ \iota_{T}=\mathrm{T}\left(\widehat{\iota_{V} \circ \psi}\right) \circ \iota_{U} \circ \varphi \\
& =\iota_{V} \circ \psi \circ \varphi=\mathrm{T}\left(\widehat{\iota_{V} \circ \psi \circ \varphi}\right) \circ \iota_{T}=\mathrm{T}\left(\mathrm{R}_{0}(\psi \circ \varphi)\right) \circ \iota_{T} .
\end{aligned}
$$

Lemma 5.2 .3 implies that

$$
\mathrm{R}_{0}(\psi) \circ \mathrm{R}_{0}(\varphi)=\mathrm{R}_{0}(\psi \circ \varphi) .
$$

Thus the composition is preserved by the assignment. One can easily check that identity morphisms are preserved. Hence, $R_{0}:$ Trs $\longrightarrow$ Ring is a functor.

Proposition 5.2.4. The functor $\mathrm{R}_{0}$ is a left adjoint to the functor $\mathrm{T}$.

Proof. For all trusses $T$ and rings $R$ let us consider the functions 


$$
\alpha_{T, R}: \operatorname{Hom}_{\text {Ring }}\left(\mathrm{R}_{0}(T), R\right) \longrightarrow \operatorname{Hom}_{\text {Trs }}(T, \mathrm{~T}(R)), \quad f \longmapsto \mathrm{T}(f) \circ \iota_{T}
$$

We will show that these functions define a natural isomorphism of bifunctors $\alpha$ : $\operatorname{Hom}_{\text {Ring }}\left(\mathrm{R}_{0}(-),-\right) \longrightarrow \operatorname{Hom}_{\text {Trs }}(-, \mathrm{T}(-))$.

Let us consider a map $\alpha_{T, R}^{-1}: \operatorname{Hom}_{\text {Trs }}(T, \mathrm{~T}(R)) \longrightarrow \operatorname{Hom}_{\text {Ring }}\left(\mathrm{R}_{0}(T), R\right), f \longmapsto \widehat{f}$, then

$$
\begin{aligned}
& \alpha_{T, R}^{-1} \circ \alpha_{T, R}(f)=\mathrm{T} \widehat{(f) \circ \iota_{T}}=f, \\
& \alpha_{T, R} \circ \alpha_{T, R}^{-1}(f)=\mathrm{T}(\widehat{f}) \circ \iota_{T}=f,
\end{aligned}
$$

where both equalities follows by the Lemma 5.2.1. Thus $\alpha_{T, R}$ is a bijection.

For naturality, take any rings $R, S$ and trusses $T, U$ and consider homomorphisms $f: \mathrm{R}_{0}(T) \longrightarrow R, \varphi: U \longrightarrow T$ and $g: R \longrightarrow S$. Then

$$
\alpha_{U, R}\left(f \circ \widehat{\iota_{T} \circ \varphi}\right)=\mathrm{T}\left(f \circ \widehat{\iota_{T} \circ \varphi}\right) \circ \iota_{U}=\mathrm{T}(f) \circ \iota_{T} \circ \varphi=\alpha_{T, R}(f) \circ \varphi,
$$

by Lemma 5.2 .1 .

Similarly,

$$
\alpha_{T, S}(g \circ f)=\mathrm{T}(g \circ f) \circ \iota_{T}=\mathrm{T}(g) \circ \alpha_{T, R}(f),
$$

as $\mathrm{T}(g)=g$ as functions. Therefore $\alpha$ in a natural isomorphism and the extension to rings functor $\mathrm{R}_{0}$ is the left adjoint to $\mathrm{T}$.

\subsubsection{Minimal extensions to rings}

An extension of a truss $T$ into a ring $R$ is a triple $\left(T, R, \eta_{T, R}\right)$, where $\eta_{T, R}: T \rightarrow \mathrm{T}(R)$ is an injective homomorphism of trusses. The universal property in Lemma 5.2 .1 allows us to extend $\eta_{T, R}$ to the ring homomorphism from the extension $\mathrm{R}_{0}(T), \widehat{\eta_{T, R}}: \mathrm{R}_{0}(T) \rightarrow R$. Now, with a ring homomorphism we can consider a kernel. Since kernel is an ideal, we can classify extensions of a truss $T$ into rings by considering the ideals in $\mathrm{R}_{0}(T)$, i.e. for all rings $R$, we can consider $\operatorname{ker}\left(\widehat{\eta_{T, R}}\right)$. This observation is used to introduce a small extensions of trusses into rings. Moreover, we identify a particular small extensions 
called minimal. Minimal extensions are universal in the sense that if minimal extensions exist, then they are unique up to isomorphism.

Definition 5.2.5. Let $T$ be a truss, $R$ a ring and let $\eta_{R}: T \longrightarrow \mathrm{T}(R)$ be an injective homomorphism of trusses. We say that $R$ is a locally small extension of $T$ if there is no subring $S \subsetneq R$ such that $\eta_{R}(T) \subseteq S$.

Proposition 5.2.6. Let $T$ be a truss and $R$ be an extension of $T$ into a ring with injection $\eta_{R}: T \longrightarrow \mathrm{T}(R)$. Then $R$ is a locally small extension if and only if $R=$ $\operatorname{Im}\left(\widehat{\eta_{R}}\right) \cong \mathrm{R}_{0}(T) / \operatorname{ker}\left(\widehat{\eta_{R}}\right)$.

Proof. Let us assume that $R$ is a locally small extension of $T$ with $\eta_{R}: T \longrightarrow \mathrm{T}(R)$. By Lemma 5.2.1 there exists a unique ring homomorphism $\widehat{\eta_{R}}: \mathrm{R}_{0}(T) \longrightarrow R$ such that $\mathrm{T}\left(\widehat{\eta_{R}}\right) \circ \iota_{T}=\eta_{R}$. Consequently, $S=\operatorname{Im}\left(\widehat{\eta_{R}}\right)$ is a subring of $R$ such that $\eta_{R}(T) \subseteq S$, and hence $S=R$, by the local smallness of the extension $R$. The first isomorphism theorem for rings yields the required isomorphism.

In the converse direction, let $R=\operatorname{Im}\left(\widehat{\eta_{R}}\right)$ (or, equivalently, $R \cong \mathrm{R}_{0}(T) / \operatorname{ker}\left(\widehat{\eta_{R}}\right)$ ) and suppose that there is a subring $S$ of $R$ such that $\eta_{R}(T) \subseteq S$. Let $j: S \longmapsto R$ be the inclusion ring homomorphism and let $\eta_{S}: T \longrightarrow S$ be given by $T(j) \circ \eta_{S}=\eta_{R}$. All these maps together with the corresponding ring homomorphisms $\widehat{\eta_{R}}$ and $\widehat{\eta_{S}}$ can be fitted in the commutative diagram:

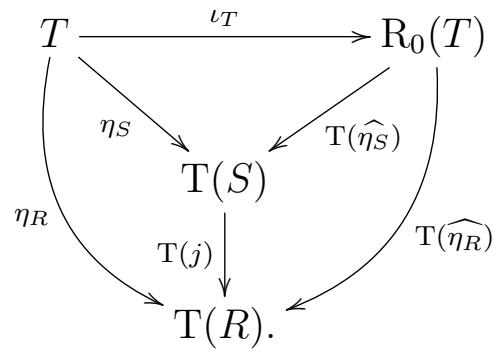

Hence $j \circ \widehat{\eta_{S}}=\widehat{\eta_{R}}$, which implies that $R=\operatorname{Im}\left(\widehat{\eta_{R}}\right) \subseteq S$, that is, $S=R$. Therefore, $R$ is a locally small ring extension of $T$.

Remark 5.2.7. Proposition 5.2.6 indicates that a locally small extension of a truss $T$ into a ring is not necessarily unique (not even up to isomorphism) and also provides 
one with a method of constructing such extensions. One needs simply to take any ring $R$ which embeds $T$ as a sub-truss of $\mathrm{T}(R)$ via an inclusion map, say, $\eta_{R}$, construct the corresponding unique ring homomorphism $\widehat{\eta_{R}}: \mathrm{R}_{0}(T) \longrightarrow R$. The ring $S=\operatorname{Im}\left(\widehat{\eta_{R}}\right) \subseteq R$ together with the corestriction of $\eta_{R}$ to $S$ is the required locally small extension (note that $\eta_{R}(T) \subseteq S$, since $\left.\widehat{\eta_{R}} \circ \iota_{T}=\eta_{R}\right)$. The form of the ring map $\widehat{\eta_{R}}: \mathrm{R}_{0}(T) \longrightarrow R$ can be easily worked out by inductive arguments. Explicitly,

$$
\widehat{\eta_{R}}:(\underbrace{[a, 0, e, 0, e, \ldots, 0, e]}_{e \text { appears } k \text {-times }}) \longmapsto \eta_{R}(a)+k \eta_{R}(e) .
$$

In particular, the map $\widehat{\iota_{T}}$ corresponding to the canonical truss inclusion $\iota_{T}: T \longrightarrow \mathrm{R}_{0}(T)$ is equal to the identity map, and hence $\mathrm{R}_{0}(T)$ is a locally small extension of $T$. In view of Proposition 5.2.6 all other locally small extensions in $T$ correspond to suitable ideals in $\mathrm{R}_{0}(T)$.

Example 5.2.8. The pair $\left(\mathbb{Q}, \iota_{\mathbb{Q}}\right)$, where $\iota_{\mathbb{Q}}: 2 \mathbb{Z}+1 \longrightarrow \mathbb{Q}, 2 k+1 \longmapsto 2 k+1$ is an extension of $2 \mathbb{Z}+1$. The ring $\mathbb{Q}$ is not locally small because $\operatorname{Im}\left(\iota_{\mathbb{Q}}\right) \subset \mathbb{Z}$ and $\mathbb{Z}$ is a subring of $\mathbb{Q}$.

Example 5.2.9. For any integer $r \geq 2$ or $r=-1$ consider the sub-truss of $T(\mathbb{Z})$,

$$
T_{r}=r(r-1) \mathbb{Z}+r=\{r((r-1) k+1) \mid k \in \mathbb{Z}\} .
$$

Note that the multiplication in $T_{r}$ is well-defined since $r^{2}$ is congruent to $r$ modulo $r(r-1)$. This truss naturally embeds in $\mathrm{T}(\mathbb{Z})$, with the embedding $\eta: n \longmapsto n$. The map $\eta$ induces a homomorphism of rings $\widehat{\eta}: \mathrm{R}_{0}\left(T_{r}\right) \longmapsto \mathbb{Z}$, which in view of Remark 5.2.7 reads

$$
\widehat{\eta}([r(r-1) k+r, 0, \underbrace{r, 0, r, \ldots, 0, r]}_{r \text { appears } l \text {-times }})=r((r-1) k+l+1),
$$

for all $k, l \in \mathbb{Z}, 0$ is the attached absorber (not zero of $\mathbb{Z}$ ), and where $l$ denotes the length of the tails. A good choice for tails is to choose the zero of $\mathbb{Z}$ and the absorber. Hence the ring $r \mathbb{Z}=\operatorname{Im}(\widehat{\eta})$ is a locally small ring extension of $T_{r}$. Since $\operatorname{Im}(\widehat{\eta}) \subsetneq \mathbb{Z}, \mathbb{Z}$ is not a locally small extension of $T_{r}$ for all $r \neq-1$.

Lemma 5.2.10. An image $\operatorname{Im}\left(\iota_{T}\right)$ is a paragon in $T_{0}$. 
Proof. Since $T_{0}$ is a two-sided truss spanned by $T \sqcup\{0\}$, and $\operatorname{Im}\left(\iota_{T}\right)$ is a $T$-submodule of $T_{0}$, it is enough to check that $\operatorname{Im}\left(\iota_{T}\right)$ is closed under induced actions of 0 . For all $t, t^{\prime} \in \operatorname{Im}\left(\iota_{T}\right)$, we have that

$0 \triangleright_{t} t^{\prime}=\left[0 t^{\prime}, 0 t, t\right]=[0,0, t]=t \in \operatorname{Im}\left(\iota_{T}\right) \quad \& \quad t_{t}^{\prime} \triangleleft 0=\left[t^{\prime} 0, t 0, t\right]=[0,0, t]=t \in \operatorname{Im}\left(\iota_{T}\right)$.

Hence, $\operatorname{Im}\left(\iota_{T}\right)$ is a paragon in $T_{0}$.

Remark 5.2.11. As indicated in Remark 5.2.7 locally small extensions of a truss $T$ correspond with ideals $I$ in $\mathrm{R}_{0}(T)$. By Lemma 5.2.10 $\iota_{T}(T)$ is a paragon in $\mathrm{R}_{0}(T)$ and thus

$$
\mathrm{I}(T):=\tau_{t}^{0}\left(\iota_{T}(T)\right)=\{[s, t, 0] \mid s \in T\}
$$

is an ideal in the extension $\mathrm{R}_{0}(T)$. To ensure that the composite map

$$
T \longleftrightarrow \mathrm{I}(T) \longleftrightarrow \mathrm{R}_{0}(T) \longrightarrow \mathrm{R}_{0}(T) / I
$$

is an injective map, we need to require that $I$ intersects trivially with $\mathrm{I}(T)$. In summary, we can state.

Remark 5.2.12. The definition of $\mathrm{I}(T)$ does not depend on $t$, as $\left[s, t^{\prime}, 0\right]=\left[\left[s, t^{\prime}, t\right], t, 0\right]$.

Lemma 5.2.13. Let $T$ be a truss, $I$ be an ideal in $\mathrm{R}_{0}(T)$ and $\pi: \mathrm{R}_{0}(T) \longrightarrow \mathrm{R}_{0}(T) / I$ be a canonical epimorphism. Then $\mathrm{R}_{0}(T) / I$ is a locally small extension of $T$ into a ring with an injection

$$
\pi \circ \iota_{T}: T \longrightarrow \mathrm{R}_{0}(T) / I
$$

if and only if $I \cap \mathrm{I}(T)=\{0\}$.

Proof. Let us assume that $\mathrm{R}_{0}(T) / I$ is a locally small extension of $T$ with an injection $\pi \circ \iota_{T}: T \longrightarrow \mathrm{R}_{0}(T) / I$, and that $I \cap \mathrm{I}(T) \neq\{0\}$. Then there exists $a \in I \cap \mathrm{I}(T) \backslash\{0\}$ and there exist $t, t \in T, t \neq t^{\prime}$, such that $\left[t, t^{\prime}, 0\right]=a$ by the definition of $\mathrm{I}(T)$. Hence,

$$
\pi(0)=\pi(a)=\pi\left(\left[t^{\prime}, t, 0\right]\right)=\left[\pi\left(t^{\prime}\right), \pi(t), \pi(0)\right] \& \pi(t)=\pi\left(t^{\prime}\right)
$$


and $\pi \circ \iota_{T}(t)=\pi(t)=\pi\left(t^{\prime}\right)=\pi \circ \iota_{T}\left(t^{\prime}\right)$, but $\pi \circ \iota_{T}$ is injective, so $t=t^{\prime}$ and $a=0$. Thus, $I \cap \mathrm{I}(T)=\{0\}$.

In the opposite direction. Let us assume that $I \cap \mathrm{I}(T)=\{0\}$. If $\pi \circ \iota_{T}(t)=\pi \circ \iota_{T}\left(t^{\prime}\right)$, then $\left[\pi(t), \pi\left(t^{\prime}\right), 0\right]=\pi\left(\left[t, t^{\prime}, 0\right]\right)=0$, by the injectivity of $\iota_{T}$. This implies that $\left[t, t^{\prime}, 0\right] \in I \cap \mathrm{I}(T)$, so $t=t^{\prime}$ and $\pi \circ \iota_{T}$ is injective. The extension $\mathrm{R}_{0}(T) / I$ is locally small by Proposition 5.2.6, as $\widehat{\pi \circ \iota_{T}}=\pi \circ \iota_{T}$.

Lemma 5.2.14. Let $T$ be a truss, then $R(T ; e)=\left(\mathrm{G}\left(T,+_{e}\right), \bullet\right)$, where for all $a, b \in T$,

$$
a \bullet b=\left[a \triangleright_{e} b, e \triangleright_{e} b, e\right]=\left[a e_{e} b, a e^{\triangleleft} e, e\right]=a b-{ }_{e} a e-{ }_{e} e b+{ }_{e} e^{2},
$$

for a fixed $e \in T$, is a ring.

Proof. In order to avoid unwieldy expressions that are too hard to read with ease, in what follows we will suppress the indexes $e$ in expressions for products, sums and actions, and keep them only in places where an action induced by a different element appears.

First we check the equality of two expressions for $\bullet$ in equation $(5.2 .2)$. This follows by the fact that $T$ is an Abelian heap,

$$
[a \triangleright b, e \triangleright b, e]=\left[a b, a e, e, e b, e^{2}, e, e\right]=\left[a b, e b, e, a e, e^{2}, e, e\right]=[a \triangleleft b, a \triangleleft e, e]
$$

As a consequence of this equality the operation $\bullet$ is a binary operation on $T$ in both cases; if $T$ is left-closed we use the left actions and when $T$ is right-closed we use the right ones.

The distributive law for $\bullet$ over + follows by the distributive laws of actions, by the absorption rules 4.1.2 and the fact that $T$ is abelian. Explicitly, for all $a, b, c \in T$,

$$
\begin{aligned}
a \bullet(b+c) & =a \bullet[b, e, c]=[a \triangleright[b, e, c], e \triangleright[b, e, c], e] \\
& =[a \triangleright b, e, a \triangleright c, e \triangleright b, e, e \triangleright c, e] \\
& =[a \triangleright b, e \triangleright b, a \triangleright c, e, e, e \triangleright c, e] \\
& =[a \triangleright b, e \triangleright b, e, e, a \triangleright c, e \triangleright c, e]=a \bullet b+a \bullet c .
\end{aligned}
$$


The right distributive law follows by symmetry through expressing the multiplication in terms of the right induced action. Finally, the associative law for $\bullet$ is a consequence of the possibility of expressing of this operation in two different ways in 5.2 .2 and the bimodule associative law in Definition 4.1. Explicitly, for all $a, b, c \in T$,

$$
\begin{aligned}
a \bullet(b \bullet c) & =[a \triangleright(b \bullet c), e \triangleright(b \bullet c), e] \\
& =[a \triangleright b \triangleleft c, a \triangleright b \triangleleft e, a \triangleright e, e \triangleright b \triangleleft c, e \triangleright b \triangleleft e, e \triangleright e, e] \\
& =[a \triangleright b \triangleleft c, a \triangleright b \triangleleft e, e, e \triangleright b \triangleleft c, e \triangleright b \triangleleft e]
\end{aligned}
$$

On the other hand,

$$
\begin{aligned}
(a \bullet b) \bullet c & =[(a \bullet b) \triangleleft c,(a \bullet b) \triangleleft e, e] \\
& =[a \triangleright b \triangleleft c, e \triangleright b \triangleleft c, e \triangleleft c, a \triangleright b \triangleleft e, e \triangleright b \triangleleft e, e \triangleleft e, e] \\
& =[a \triangleright b \triangleleft c, e \triangleright b \triangleleft c, e, a \triangleright b \triangleleft e, e \triangleright b \triangleleft e] \\
& =[a \triangleright b \triangleleft c, a \triangleright b \triangleleft e, e, e \triangleright b \triangleleft c, e \triangleright b \triangleleft e]=a \bullet(b \bullet c),
\end{aligned}
$$

as required.

Lemma 5.2.15. Let $T$ be a truss. Then $R(T ; e) \cong \mathrm{I}(T)$.

Proof. Obviously, $\mathrm{T}(R(T ; e))=T$ is a sub-heap of $T_{0}, \tau_{e}^{0}: \mathrm{T}(R(T ; e)) \rightarrow \mathrm{I}(T)$ is an isomorphism of heaps and $\tau_{e}^{0}(e)=0$. Moreover, for all $a, b \in T$

$$
\begin{aligned}
\tau_{e}^{0}(a) \cdot \tau_{e}^{0}(b) & =[a, e, 0][b, e, 0]=\left[a b, e b, 0, a e, e^{2}, 0,0\right]=\left[a b, e b, e^{2}, a e, 0\right] \\
& =\left[a b, e b, e^{2}, a e, e, e, 0\right]=[a \bullet b, e, 0]=\tau_{e}^{0}(a \bullet b),
\end{aligned}
$$

where the second equality follows by distributive laws, third and forth by Mal'cev identities. Thus, since $\tau_{e}^{0}$ is an isomorphism of heaps which preserves neutral elements of retracts and multiplications, $\tau_{e}^{0}$ is the isomorphism of rings.

Corollary 5.2.16. For all $p, p^{\prime} \in T, R(T ; p)$ and $R\left(T ; p^{\prime}\right)$ are isomorphic rings.

Proof. Observe that for all $p \in T R(T ; p) \cong \tau_{p}^{0}(T)$, thus

$$
R(T ; p) \cong \tau_{p}^{0}(T)=\tau_{p^{\prime}}^{0}(T) \cong R\left(T ; p^{\prime}\right),
$$

for all $p^{\prime} \in T$. 
Lemma 5.2.17. Let $T$ be a truss with finite exponent $N \in \mathbb{N}$, then the sub-heap $I_{N}=\{\underbrace{[0, e, 0, e, \ldots, 0, e, 0]}_{e \text { appears } k \text {-times }}, \underbrace{[e, 0, e, 0, \ldots, e, 0, e]}_{e \text { appears } k \text {-times }} \mid k=n N, n \in \mathbb{N} \cup\{0\}\} \subseteq T_{0}$ is an ideal.

Proof. It is easy to check that $I_{N}$ is a sub-heap of $T_{0}$. Let $[e, 0, \ldots, e] \in I_{N}$ be an element of length $2 h N+1$ for some $h \in \mathbb{N}$. Then for all $t \in T$,

$$
\begin{aligned}
0[e, 0 \ldots, e] & =0 \in I_{N} \&[e, 0 \ldots, e] 0=0 \in I_{N}, \\
t[e, 0 \ldots, e] & =[t e, 0, \ldots, t e]=[[t e, 0, \ldots, t e], \underbrace{[e, \ldots, e]}_{2 h N-1}, e] \\
& =[[t e, e, \ldots, t e],[0, e, \ldots, 0], e]=[e, 0, \ldots, 0, e] \in I_{N}, \\
{[e, 0 \ldots, e] t } & =[e t, 0, \ldots, e t]=[[e t, 0, \ldots, e t], \underbrace{[e, \ldots, e]}_{2 h N-1}, e] \\
& =[[e t, e, \ldots, e t],[0, e, \ldots, 0], e]=[e, 0, \ldots, 0, e] \in I_{N} .
\end{aligned}
$$

Thus, elements of the form $[e, 0 \ldots, e]$ are closed under multiplication by generators of $T_{0}$. Now, since $[0, e, 0, e, \ldots, 0, e, 0]=[0,[e, 0, e, \ldots, 0, e], 0]$ and $I_{N}$ is a sub-heap of $T$, $I_{N}$ is an ideal in $T_{0}$. Thus $\mathrm{G}\left(I_{N} ; 0\right)$ is an ideal in $\mathrm{R}_{0}(T)$.

In the hierarchy of locally small extensions of a truss $T$ one can distinguish those that are particularly close to $T$.

Definition 5.2.18. A locally small extension $\left(S, \eta_{S}\right)$ of a truss $T$ is called a small extension provided $\widehat{\eta_{S}}(\mathrm{I}(T))$ is an essential ideal in $S$, i.e. for all ideals $\{0\} \neq I \triangleleft S$, $\widehat{\eta_{S}}(\mathrm{I}(T)) \cap I \neq\{0\}$.

Corollary 5.2.19. Let $T$ be a truss such that the (any) retract $\mathrm{G}(T ;$ e) has a finite exponent. Then the extension $\mathrm{R}_{0}(T) / I_{N}$ is a locally small extension of $T$.

Proof. It follows straight forward by Lemma 5.2.13.

Taking into account the explicit form of the induced ring map $\widehat{\eta_{S}}$ described in Remark 5.2.7 one immediately obtains the following characterisation of small extensions. 
Lemma 5.2.20. Let $T$ be a truss, $e \in T$ and let $\left(S, \eta_{S}\right)$ be a locally small extension of $T$. Then $\left(S, \eta_{S}\right)$ is a small extension if and only if, for all ideals $\{0\} \neq J \triangleleft S$ there exists $a \in T$ such that $a \neq e$ and $\eta_{S}(a)-\eta_{S}(e) \in J$.

Proof. Let $\left(S, \eta_{S}\right)$ be a small extension of $T$. Then for all $\{0\} \neq J \triangleleft S$ there exists $j \in \mathrm{I}(T)$ such that $\widehat{\eta_{S}}(j) \neq 0$ and $\widehat{\eta_{S}}(j) \in J \cap \widehat{\eta_{S}}(\mathrm{I}(T))$. Thus there exists $a \in T$ such that $j=[a, e, 0]$. Hence,

$$
\eta_{S}(a)-\eta_{S}(e)=\widehat{\eta_{S}}(a)-\widehat{\eta_{S}}(e)=\widehat{\eta_{S}}([a, e, 0])=\widehat{\eta_{S}}(j) \in J .
$$

In the opposite direction. If $a \neq e, \eta_{S}$ is injective and $\eta_{S}(a)-\eta_{S}(e) \in J$, then

$$
\eta_{S}(a)-\eta_{S}(e)=\left[\eta_{S}(a), \eta_{S}(e), 0\right]=\left[\widehat{\eta_{S}}(a), \widehat{\eta_{S}}(e), \widehat{\eta_{S}}(0)\right]=\widehat{\eta_{S}}([a, e, 0]) \neq 0 \in J .
$$

Thus, since $\widehat{\eta_{S}}([a, e, 0]) \in \widehat{\eta_{S}}(\mathrm{I}(T))$, we get that $\widehat{\eta_{S}}([a, e, 0]) \in J \cap \widehat{\eta_{S}}(\mathrm{I}(T))$, and $\widehat{\eta_{S}}(\mathrm{I}(T))$ is an essential ideal in $S$.

Example 5.2.21. Let $T_{r}$ be the truss defined in Example 5.2.9. Consider the locally small extension $\eta: T_{r} \longrightarrow \mathrm{T}(r \mathbb{Z}), n \mapsto n$ (with $e=r$ ). Since $r \mathbb{Z}$ is a principal ideal domain, all ideals in $r \mathbb{Z}$ are of the form $I_{q}=q r \mathbb{Z}$, for a non-negative integer $q$. Then

$$
I_{q} \ni q r(r-1)=\eta(r(r-1) q+r)-\eta(r),
$$

and hence $r \mathbb{Z}$ is a small extension of $T_{r}$.

There exist locally small extensions which are not small extension. As we observed in Remark 5.2.7 $\mathrm{R}_{0}(T)$ is a locally small extension of a truss $T$ that usually is not small. For example if $T=\mathrm{T}(R)$, for some ring $R$ with a zero $0_{R}$, then $\iota_{R}(R)_{0_{R}}^{0} \subseteq \mathrm{R}_{0}(T)$ and clearly $\iota_{R}(R)_{0_{R}}^{0}$ is not an essential ideal in $\mathrm{R}_{0}(T)$. Similarly, if $(R,+)$ has a finite exponent $N$.

Finally we look at extensions which are at the bottom of the hierarchy of locally small extensions.

Definition 5.2.22. Let $T$ be a truss and $\left(S, \eta_{S}\right)$ be a locally small extension of $T$ into a ring $S$. Then we say that $\left(S, \eta_{S}\right)$ is a minimal extension if for all ideals $I \subseteq \mathrm{R}_{0}(T)$ such that $I \cap \mathrm{I}(T)=\{0\}, I \subseteq \operatorname{ker}\left(\widehat{\eta_{S}}\right)$. 
Lemma 5.2.23. A minimal extension of a truss is unique up to isomorphism.

Proof. Let $\left(S, \eta_{S}\right)$ and $\left(S^{\prime}, \eta_{S^{\prime}}\right)$ be two minimal extensions of a truss $T$. Then $\operatorname{ker}\left(\widehat{\eta_{S}}\right) \subseteq$ $\operatorname{ker}\left(\widehat{\eta_{S^{\prime}}}\right)$ and $\operatorname{ker}\left(\widehat{\eta_{S^{\prime}}}\right) \subseteq \operatorname{ker}\left(\widehat{\eta_{S}}\right)$, so $\operatorname{ker}\left(\widehat{\eta_{S}}\right)=\operatorname{ker}\left(\widehat{\eta_{S^{\prime}}}\right)$, and isomorphism is given by the first isomorphism theorem for rings.

Example 5.2.24 (Minimal extensions exist). Let $T=\mathrm{T}(R)$ for a ring $R$, and let us fix an element $0_{R} \in \mathrm{T}(R)$, then $R$ is a minimal extension of $\mathrm{T}(R)$ to a ring. Observe that the ring homomorphism $\widehat{\eta}: \mathrm{R}_{0}(T) \longrightarrow R$ induced from the identity map $\eta: R \longrightarrow R$ is onto. Moreover, the composition $\mathrm{T}(\widehat{\eta}) \circ \iota_{T}: T(R) \rightarrow T(R)$ is an isomorphism. Therefore $R$ is a minimal extension of itself to a ring.

Observe, that if $\left(S, \eta_{S}\right)$ is a small extension, then it is a locally small extension. Analogously, every minimal extension is a small extension. This easily follows by the definitions of locally small, small and minimal extensions.

Lemma 5.2.25. Let $\left(S, \eta_{S}\right)$ be an extension of a truss $T$ with at least two elements such that $S$ is a domain. If $\left(S, \eta_{S}\right)$ is a small extension, then $\left(S, \eta_{S}\right)$ is a minimal extension.

Proof. Let $I$ be an ideal in $\mathrm{R}_{0}(T)$ such that $I \cap \mathrm{I}(T)=\{0\}$, then $0 \in I$. By Proposition 1.4.1 there are only three types of words in $\mathrm{R}_{0}(T)$ that can be in $I$,

$\left[t_{1}, t_{2}, 0\right],\left[t_{1} 0, t_{2}, 0, \ldots, t_{n}\right]$ and $\left[0, t_{1}, 0, \ldots, t_{n}, 0\right]$ for $n \in \mathbb{N}$ and $t_{1}, \ldots, t_{n} \in T$.

As the intersection is empty and $\left[t_{1}, t, 0\right] \in \mathrm{I}(T)$,

$$
\left[t_{1}, t, 0\right] \in I \Longleftrightarrow t_{1}=t
$$

Since $0 \in I \cap \operatorname{ker}\left(\widehat{\eta_{S}}\right)$,

$$
\begin{aligned}
{\left[t_{1}, 0, t_{2}, 0, \ldots, t_{n}\right] \in \operatorname{ker}\left(\widehat{\eta_{S}}\right) } & \Longleftrightarrow\left[0, t_{1}, 0, \ldots, t_{n}, 0\right] \in \operatorname{ker}\left(\widehat{\eta_{S}}\right) \\
{\left[t_{1}, 0, t_{2}, 0, \ldots, t_{n}\right] \in I } & \Longleftrightarrow\left[0, t_{1}, 0, \ldots, t_{n}, 0\right] \in I
\end{aligned}
$$


Thus, if $\left[t_{1}, 0, t_{2}, 0, \ldots, t_{n}\right] \in I$, then for all $t \neq t^{\prime} \in T$,

$$
\left[t, t^{\prime}, 0\right]\left[t_{1}, 0, t_{2}, 0, \ldots, t_{n}\right]=\left[\left[t t_{1}, t^{\prime} t_{1}, t t_{2}, t^{\prime} t_{2}, \ldots, t t_{n}\right], t^{\prime} t_{n}, 0\right] \in I \cap \mathrm{I}(T)=\{0\} .
$$

Hence,

$$
\widehat{\eta_{S}}\left(\left[t, t^{\prime}, 0\right]\left[t_{1}, 0, t_{2}, 0, \ldots, t_{n}\right]\right)=\widehat{\eta_{S}}\left(\left[t, t^{\prime}, 0\right]\right) \widehat{\eta_{S}}\left(\left[t_{1}, 0, t_{2}, 0, \ldots, t_{n}\right]\right)=\widehat{\eta_{S}}(0)=0
$$

Since $S$ is a domain, $\widehat{\eta_{S}}(t)=\widehat{\eta_{S}}\left(t^{\prime}\right)$ or $\widehat{\eta_{S}}\left(\left[t_{1}, 0, t_{2}, 0, \ldots, t_{n}\right]\right)=0$. Therefore, since $\widehat{\eta_{S}}$ is injective on $T$, and $t \neq t^{\prime}$, we get that $\left[t_{1}, 0, t_{2}, 0, \ldots, t_{n}\right] \in \operatorname{ker}\left(\widehat{\eta_{S}}\right)$. Thus $I \subseteq \operatorname{ker}\left(\widehat{\eta_{S}}\right)$, and $\left(S, \eta_{S}\right)$ is a minimal extension.

Example 5.2.26. Let us consider the $T_{r}=r(r-1) \mathbb{Z}+r$, for $r=-1$ or $r \geq 2$ of Example 5.2.9. By Example 5.2.21 $T_{r} \hookrightarrow \mathrm{T}(r \mathbb{Z})$ is a small extension and since $r \mathbb{Z}$ is a domain, $r \mathbb{Z}$ is a minimal extension of $T_{r}$ by Lemma 5.2.25.

Example 5.2.27 (Small but not minimal extension). Let $p$ be a prime number and consider the truss

$$
T=\left(\begin{array}{cc}
1 & \mathbb{Z}_{p} \\
0 & 1
\end{array}\right)
$$

with the usual matrix multiplication and the heap structure arising from the matrix addition. For $e=\left(\begin{array}{ll}1 & 0 \\ 0 & 1\end{array}\right)$, the extension $\mathrm{R}_{0}(T)$ can be identified with

$$
\mathrm{R}_{0}(T)=\left\{\left(\begin{array}{cc}
m & a \\
0 & m
\end{array}\right) \mid m \in \mathbb{Z}, a \in \mathbb{Z}_{p}\right\},
$$

a Dorroh extension of the ring $\mathbb{Z}_{p}$ with zero multiplication. With this identification, $\iota_{T}: T \longrightarrow \mathrm{R}_{0}(T)$ is the obvious (set-theoretic) inclusion map, and the corresponding ideal $\mathrm{I}(T)$ of $\mathrm{R}_{0}(T)$ comes out as

$$
\mathrm{I}(T)=\left(\begin{array}{cc}
0 & \mathbb{Z}_{p} \\
0 & 0
\end{array}\right)
$$

For all $n \in \mathbb{N}$, let us define injective truss homomorphisms

$$
\eta_{n}: T \longrightarrow \mathrm{T}\left(\mathbb{Z}_{p^{n+1}}\right), \quad\left(\begin{array}{ll}
1 & a \\
0 & 1
\end{array}\right) \longmapsto\left(1-a p^{n}\right) \quad\left(\bmod p^{n+1}\right) .
$$


The universally constructed ring homomorphisms are

$$
\widehat{\eta}_{n}: \mathrm{R}_{0}(T) \longrightarrow \mathbb{Z}_{p^{n+1}}, \quad\left(\begin{array}{cc}
m & a \\
0 & m
\end{array}\right) \longmapsto\left(m-a p^{n}\right) \quad\left(\bmod p^{n+1}\right) .
$$

Each of the maps $\widehat{\eta_{n}}$ is onto so the extensions $\eta_{n}: T \longrightarrow \mathbb{Z}_{p^{n+1}}$ are locally small. Furthermore, since for all $n$, the ideals

$$
\widehat{\eta}_{n}(\mathrm{I}(T))=\left\{a p^{n} \quad\left(\bmod p^{n+1}\right) \mid a \in \mathbb{Z}_{p}\right\},
$$

are essential in $\mathbb{Z}_{p^{n+1}}$, all these extensions are small. By the uniqueness of the minimal extensions at most one of them could be minimal. Thus we obtain an infinite family of small extensions that are not minimal.

Remark 5.2.28. In [36, K.I. Beidar] introduced and investigated maximal essential extensions of rings, see Definition B.2.12. In contrast, in this chapter we consider small and minimal extensions of trusses. These can be interpreted as essential extensions of rings if we consider ring-type trusses, see Lemma 2.1.6. We consider a truss $\mathrm{T}(R)$ associated with a ring $R$. Then all extensions of a truss $\mathrm{T}(R)$ are extensions of a ring $R$. Sadly, in this case minimality trivialises, as every ring is a minimal essential extension of itself. The issue is how a truss is assigned to a ring. We introduce another approach. Let us consider a ring $S$ and its extension $E$. Let us assume that $S$ is a subring of $E$ and that there exists an idempotent $a \in E \backslash S$, i.e. $a^{2}=a$, such that $a S=S=S a$. For any ring $S$ we can consider such an extension, for example, by taking a Dorroh extension, the unitalisation of a ring. Then with every $S$ we can associate a truss $a+S$, a coset of the underlying normal subgroup of $S$ in $E$. This is a truss and $R(a+S ; a) \cong S$, see the definition of the ring operation in Lemma 5.2.14. Now, by Proposition 5.1.10 we can construct a ring $\mathrm{R}_{0}(a+S)$. Since $E$ is an extension of $a+S$ into a ring, by Lemma 5.2.15. $\mathrm{I}(a+S) \cong S$. Thus for any extension of a truss $a+S$ into a ring $E^{\prime}$, we get an extension of a ring $\mathrm{I}(a+S) \cong S$. If $E^{\prime}$ is a small extension, then $E^{\prime}$ is an essential extension of $\mathrm{I}(a+S)$, see Definition B.2.11. Moreover, if we assume $E^{\prime}$ is minimal we get some kind of a unique extension in some particular class of extensions of ring $S$, a subclass of essential extensions. It seems that additional properties of the idempotent $a$ have a special meaning here. Observe that in the case of a truss associated with Dorroh 
extension, in this way, we get some kind of a "minimal essential unital extension". This approach works for any ring $S$. We conclude this remark with a small example, in this setting, the minimal essential extension of a ring $2 \mathbb{Z}$ by Dorroh extension is $\mathbb{Z}$. 


\section{Chapter 6}

\section{On the categories of modules}

This chapter is focused on the categories of heaps and $T$-modules. Let $T$ be a truss

and $R$ be a ring. We study functors among categories of Abelian heaps $\mathbf{A h}, R$-modules $R$-mod, $T$-modules $T$-mod and unital $T$-modules $T_{1}$-mod. Modules over rings will only appear in Section 6.2. There are three aims of this chapter. First, to establish connection between categories $T$ - $\bmod$ and $\left(T_{u}\right)_{1}$-mod, where $T_{u}$ is the unital extension of a truss $T$ from Proposition 5.1.11. Second aim is to describe a free object in $T$-mod. Third aim is to construct a tensor product for appropriate bimodules, and heaps. By accomplishing all the aims we will achieve a necessary background to introduce a Morita theory for trusses.

In Section 6.1, we show that a category $T$-mod of modules over a truss $T$ is isomorphic with a category $\left(T_{u}\right)_{1}$-mod of modules over a unital extension $T_{u}$.

In Section 6.2, we introduce the definition of a free unital $T$-module. Further, we study connections between free modules over a ring $R$ and free module over an associated truss $\mathrm{T}(R)$.

In Section 6.3, we construct and study tensor product of bimodules over trusses.

Sections 6.1 and 6.3 are Sections 2.5 and 4 of [33], respectively. Section 6.2 is Section 4 of [19]. 


\subsection{Unital trusses and trusses}

Recall from Proposition 5.1 .11 that by $T_{u}$ we denote the truss $T \boxplus\{1\}$.

Proposition 6.1.1. Let $T$ be a truss. The heap homomorphism $\iota_{T}: T \longrightarrow T_{u}, t \longmapsto t$, is a morphism of trusses. Furthermore, if $S$ is a unital truss, then for every morphism of trusses $f: T \longrightarrow S$ there exists a unique morphism of unital trusses $\tilde{f}: T_{u} \longrightarrow S$, such that $\tilde{f} \circ \iota_{T}=f$.

Proof. Let us assume that $S$ is a unital truss and that $f: T \longrightarrow S$ is a truss homomorphism. If we consider the heap map $\eta:\{1\} \longrightarrow S, 1 \longmapsto 1_{S}$, then there exists a unique morphism of Abelian heaps $\tilde{f}: T \boxplus\{1\} \longrightarrow S$ such that $\tilde{f} \circ \iota_{T}=f$ and $\tilde{f} \circ \iota_{\{1\}}=\eta$. We claim that $\tilde{f}$ is a morphism of unital trusses. Unitality follows by definition, since $\tilde{f}(1)=\eta(1)=1_{S}$. To check multiplicativity pick two symmetric words $\left[a_{1}, a_{2}, a_{3}, \ldots, a_{2 k}, a_{2 k+1}\right]$ and $\left[b_{1}, b_{2}, b_{3}, \ldots, b_{2 h}, b_{2 h+1}\right]$ in $T \boxplus\{1\}$, where the symbols $a_{i}, b_{j}$ belongs to $T \sqcup\{1\}$ for all $i, j$. Since we have that

$$
\begin{gathered}
\tilde{f}\left(\left[a_{1}, a_{2}, a_{3}, \ldots, a_{2 k}, a_{2 k+1}\right] \cdot\left[b_{1}, b_{2}, b_{3}, \ldots, b_{2 h}, b_{2 h+1}\right]\right)= \\
=\tilde{f}\left(\left[\left(a_{1} \cdot b_{1}\right),\left(a_{1} \cdot b_{2}\right), \ldots,\left(a_{1} \cdot b_{2 h+1}\right),\left(a_{2} \cdot b_{1}\right), \ldots,\left(a_{i} \cdot b_{j}\right), \ldots,\left(a_{2 k+1} \cdot b_{2 h+1}\right)\right]\right) \\
=\left[\tilde{f}\left(a_{1} \cdot b_{1}\right), \tilde{f}\left(a_{1} \cdot b_{2}\right), \ldots, \tilde{f}\left(a_{1} \cdot b_{2 h+1}\right), \tilde{f}\left(a_{2} \cdot b_{1}\right), \ldots, \tilde{f}\left(a_{i} \cdot b_{j}\right), \ldots, \tilde{f}\left(a_{2 k+1} \cdot b_{2 h+1}\right)\right],
\end{gathered}
$$

it is enough to check that $\tilde{f}$ is multiplicative on a product $a \cdot b$ where $a, b \in T \sqcup\{1\}$. Now,

$$
\tilde{f}(a \cdot b)= \begin{cases}\tilde{f}(1 \cdot 1)=\tilde{f}(1)=1_{S}=1_{S} \cdot 1_{S}=\tilde{f}(1) \cdot \tilde{f}(1) & a, b \in\{1\} \\ \tilde{f}(t \cdot 1)=\tilde{f}(t)=\tilde{f}(t) \cdot 1_{S}=\tilde{f}(t) \cdot \tilde{f}(1) & a \in T, b \in\{1\} \\ \tilde{f}(1 \cdot t)=\tilde{f}(t)=1_{S} \cdot \tilde{f}(t)=\tilde{f}(1) \cdot \tilde{f}(t) & a \in\{1\}, b \in T \\ \tilde{f}\left(t \cdot t^{\prime}\right)=\tilde{f}\left(t t^{\prime}\right)=f\left(t t^{\prime}\right)=f(t) \cdot f\left(t^{\prime}\right)=\tilde{f}(t) \cdot \tilde{f}\left(t^{\prime}\right) & a, b \in T\end{cases}
$$

that is, $\tilde{f}(a \cdot b)=\tilde{f}(a) \cdot \tilde{f}(b)$ for all $a, b \in T \sqcup\{1\}$ and the proof is complete.

Theorem 6.1.2. Let $T$ be a truss. Any $T$-module $M$ is naturally a unital $T_{u}$-module. This induces a functor $\mathrm{E}_{1}: T-\bmod \longrightarrow\left(T_{u}\right)_{1}$-mod which is the inverse of the restriction of scalars functor $\mathrm{S}_{T}:\left(T_{u}\right)_{1}-\bmod \longrightarrow T$-mod along the truss homomorphism $\iota_{T}: T \rightarrow T_{u}$. In particular, we have an isomorphism of categories $\left(T_{u}\right)_{1}$-mod $\cong T$-mod. 
Proof. Observe that to equip an Abelian heap $M$ with the structure of a $T$-module is the same as to define a truss homomorphism $\rho_{M}: T \longrightarrow E(M)$, see Example 2.1.14 and Remark 4.1.3. Since $E(M)$ is unital with unit $1_{M}, \rho$ extends uniquely to a unital truss homomorphism $\tilde{\rho}_{M}: T_{u} \longrightarrow E(M)$ by Proposition 6.1.1, making of $M$ a unital $T_{u}$-module. Let $f: M \rightarrow N$ be a morphism of $T$-modules. To check that it is $T_{u}$-linear as well, observe that $f$ is $T_{u}$-linear if and only if the following diagram commutes

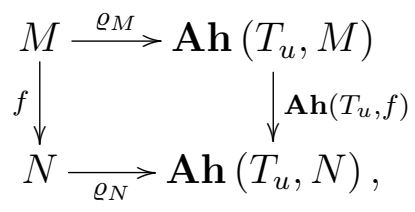

where $\varrho_{M}(m): z \longmapsto \tilde{\rho}_{M}(z)(m)$ and $\mathbf{A h}\left(T_{u}, f\right): g \longmapsto f \circ g$. Therefore, we are led to check that, for all $m \in M$,

$$
f \circ \varrho_{M}(m)=\varrho_{N}(f(m))
$$

as heap homomorphisms from $T_{u}$ to $N$. However, since

$$
\begin{aligned}
\left(f \circ \varrho_{M}(m) \circ \iota_{\{1\}}\right)(1) & =f\left(\varrho_{M}(m)\left(\iota_{\{1\}}(1)\right)\right)=f\left(\tilde{\rho}_{M}\left(\iota_{\{1\}}(1)\right)(m)\right)=f(m) \\
& =\tilde{\rho}_{N}\left(\iota_{\{1\}}(1)\right)(f(m))=\left(\varrho_{N}(f(m)) \circ \iota_{\{1\}}\right)(1), \\
\left(f \circ \varrho_{M}(m) \circ \iota_{T}\right)\left(\iota_{T}(t)\right) & =f\left(\varrho_{M}(m)\left(\iota_{T}(t)\right)\right)=f\left(\tilde{\rho}_{M}\left(\iota_{T}(t)\right)(m)\right)=f\left(\rho_{M}(t)(m)\right) \\
& =\rho_{N}(t)(f(m))=\tilde{\rho}_{N}\left(\iota_{T}(t)\right)(f(m))=\left(\varrho_{N}(f(m)) \circ \iota_{T}\right)(t),
\end{aligned}
$$

for all $t \in T$, it follows by the universal property of the direct sum that 6.1.1) holds. Summing up, there is a fully faithful functor

$$
\mathrm{E}_{1}: T-\bmod \longrightarrow\left(T_{u}\right)_{1} \text {-mod, } \quad\left\{\begin{array}{l}
M \longmapsto M \\
f \longmapsto f
\end{array} .\right.
$$

Now, if $\left(M, \rho_{M}\right)$ is a $T$-module and we consider its unital extension $\left(\mathrm{E}_{1}(M), \tilde{\rho}_{M}\right)$, then the restriction of scalars functor $\mathrm{S}_{T}$ endows $\mathrm{E}_{1}(M)$ with the $T$-module structure given by the composition

$$
T \stackrel{\iota_{T}}{\longrightarrow} T_{u} \stackrel{\tilde{\rho}_{M}}{\longrightarrow} E(M)
$$

which coincides with $\rho_{M}$ by definition of $\tilde{\rho}_{M}$. The other way around, if $\left(N, \tilde{\rho}_{N}\right)$ is a unital $T_{u}$-module and we construct the unital extension $\mathrm{E}_{1}\left(\mathrm{~S}_{T}(N)\right)$ of the $T$-module $\left(\mathrm{S}_{T}(N), \rho_{N}\right)$ obtained by restriction of scalars along $\iota_{T}$, then this is given by the unique unital extension of $\rho_{N}=\tilde{\rho}_{N} \circ \iota_{T}$ and the latter has to coincide with $\tilde{\rho}_{N}$ by uniqueness. 
As a matter of notation, in the future, we will often omit to specify the functors $\mathrm{S}_{T}$ and $\mathrm{E}_{1}$, unless their presence would increase the clarity of the exposition.

\subsection{Free modules}

Throughout this section $T$ is a unital truss. By the preceding section we know that the categories $T$-mod and $\left(T_{u}\right)_{1}$-mod are isomorphic. Thus, we can narrow our consideration to unital modules over unital trusses, keeping in mind the construction of the functor $\mathrm{S}_{T}:\left(T_{u}\right)_{1}-\bmod \rightarrow T$-mod.

Let $X$ be a set. For every $x \in X$, let us define the unital left $T$-module

$$
T x:=\{t x \mid t \in T\}, \quad\left[t x, t^{\prime} x, t^{\prime \prime} x\right]:=\left[t, t^{\prime}, t^{\prime \prime}\right] x, \quad t \cdot\left(t^{\prime} x\right)=\left(t t^{\prime}\right) x,
$$

i.e. $T x$ is a $T$-module generated by $x$ that is obviously isomorphic to $T$ (as a left module). By convention $1 x$ is identified with $x$, so that we may view $x$ as an element of $T x$. Now we can consider the direct sum module

$$
\mathcal{T}^{X}:=\bigoplus_{x \in X} T x
$$

From Proposition 1.4.1 and the construction of the coproduct of modules we observe that every element of $\mathcal{T}^{X}$ can be written as

$$
\left[t_{1} x_{1}, \ldots, t_{n} x_{n}, k_{i_{1}} x_{i_{1}}, \ldots, k_{i_{n^{\prime}}} x_{i_{n^{\prime}}}\right]
$$

where $\left\{i_{1}, \ldots, i_{n^{\prime}}\right\} \subseteq\{1, \ldots, n\}, t_{j} \in T, k_{j} \in \mathbb{Z}, x_{j} \in X, k_{j} x_{j}=[\underbrace{x_{j}, e, x_{j}, e, \ldots, x_{j}}_{x_{j} \text { appears } k_{j} \text {-times }}]$ and $n+\sum_{j=i_{1}}^{i_{n^{\prime}}} k_{j}$ is an odd number, for any fixed $e \in X$. Moreover one can observe that there are isomorphisms of heaps

$$
\begin{aligned}
\mathcal{T}^{X} & \cong \mathrm{H}\left(\mathrm{G}(T e ; e) \oplus\left(\bigoplus_{x \in X \backslash\{e\}}(\mathrm{G}(T x ; x) \oplus \mathrm{G}(\mathcal{H}(\{x\}) ; x))\right)\right) \\
& \cong \mathrm{H}\left(\mathrm{G}(T e ; e) \oplus\left(\bigoplus_{x \in X \backslash\{e\}}(\mathrm{G}(T x ; x) \oplus \mathbb{Z})\right)\right)
\end{aligned}
$$


analogous to those found in Proposition 1.4.4 and Corollary 1.4.6. Although the $T$-module structure of $\mathcal{T}^{X}$ can be transferred to the right hand side through this isomorphism, the form of the transferred action interacts nontrivially and often in a not necessarily illuminating manner with the direct sum of groups (compare 4.3.1) in the case of two modules).

Following the categorical idea of freeness (see e.g. the universal property in Lemma 1.3.1) let us fix set $X$ and consider inclusion $\iota_{X}: X \rightarrow \mathcal{T}^{X}$, given by $\iota_{X}(x)=1 x$, for all $x \in X$. Then, for any unital $T$-module $N$ and any function $\varphi: X \longrightarrow N$ we obtain the following commutative diagram

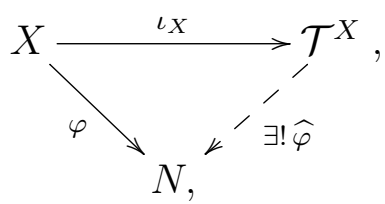

where the unique $T$-module morphism $\widehat{\varphi}$ is defined by

$$
\begin{gathered}
\widehat{\varphi}: \mathcal{T}^{X} \longrightarrow N \\
{\left[t_{1} x_{1}, \ldots, t_{n} x_{n}, k_{i_{1}} x_{i_{1}}, \ldots, k_{i_{n^{\prime}}} x_{i_{n^{\prime}}}\right]} \\
\longmapsto \\
\longmapsto\left[t_{1} \varphi\left(x_{1}\right), \ldots, t_{n} \varphi\left(x_{n}\right), k_{i_{1}} \varphi\left(x_{i_{1}}\right), \ldots, k_{i_{n^{\prime}}} \varphi\left(x_{i_{n^{\prime}}}\right)\right],
\end{gathered}
$$

where $k_{j} \varphi\left(x_{j}\right)=\left[\varphi\left(x_{j}\right), \varphi(e), \varphi\left(x_{j}\right), \varphi(e), \ldots, \varphi\left(x_{j}\right)\right]$. Since this is the universal property characterising a free object in the category of unital modules over $T, \mathcal{T}^{X}$ is the free unital $T$-module on $X$, as expected.

Following the usual ring-theoretic conventions we can formulate

Definition 6.2.1. A unital $T$-module is said to be generated by a set $X$, if there exists a $T$-module epimorphism $\mathcal{T}^{X} \longrightarrow M$. It is said to be finitely generated if there exists finite $X$ that generates $M . M$ is a (finitely generated) free $T$-module if it is isomorphic to $\mathcal{T}^{X}$, for some (finite) $X$.

As in the case of modules over a ring, one can try to characterise free modules by the existence of a basis. 
Definition 6.2.2. Let $M$ be a left $T$-module and let $X$ be a non-empty subset of $M$. Let, for all $x \in X, \sigma_{x}$ denote the left $T$-module homomorphism

$$
\sigma_{x}: T \longrightarrow M, \quad t \longmapsto t x
$$

(1) We say that the set $X$ is free if, for all finite subsets $S$ of $X$, the map $\underset{x \in S}{\boxplus} \sigma_{x}$ is a monomorphism.

(2) A free set $B$ is said to be a basis for $M$ if the map $\underset{x \in B}{\boxplus} \sigma_{x}$ is an epimorphism.

Lemma 6.2.3. If $X$ is a free subset of $M$, then, for all finite non-empty proper subsets $Y$ of $X$ and all $x \in X \backslash Y$,

$$
\sigma_{x}(T) \cap\left(\underset{y \in Y}{\boxplus} \sigma_{y}\right)(\underset{Y}{\boxplus} T)=\emptyset
$$

Proof. Set $V=\underset{y \in Y}{\boxplus} T$ and $\sigma_{Y}=\underset{y \in Y}{\boxplus} \sigma_{y}$, and suppose that there exist $m \in M, t \in T$ and $v \in V$ such that $\sigma_{x}(t)=\sigma_{Y}(v)=m$. Take any $v^{\prime} \in V$. In view of Proposition 1.4.1 the words $\overline{: t v v^{\prime}:}$ and $\overline{: v t v^{\prime}:}$ are different, but

$$
\begin{aligned}
& \left(\sigma_{x} \boxplus \sigma_{Y}\right)\left(\overline{: t v v^{\prime}:}\right)=\left[\sigma_{x}(t), \sigma_{Y}(v), \sigma_{Y}\left(v^{\prime}\right)\right]=\left[m, m, \sigma_{Y}\left(v^{\prime}\right)\right]=\sigma_{Y}\left(v^{\prime}\right), \\
& \left(\sigma_{x} \boxplus \sigma_{Y}\right)\left(\overline{: v t v^{\prime}:}\right)=\left[\sigma_{Y}(v), \sigma_{x}(t), \sigma_{Y}\left(v^{\prime}\right)\right]=\left[m, m, \sigma_{Y}\left(v^{\prime}\right)\right]=\sigma_{Y}\left(v^{\prime}\right),
\end{aligned}
$$

which contradicts the assumption that $\sigma_{x} \boxplus \sigma_{Y}$ is a monomorphism.

The statement of Lemma 6.2.3 is in perfect categorical accord with what might be expected of a free or a linearly independent set. Just as in the case of modules or vector spaces, the intersection of the module spanned by any finite subset of a free set with a cyclic module generated by an element from within the free set but without this subset is the zero module, i.e. the initial object in the category of modules, so is the corresponding intersection in the case of modules over a truss - the empty set, i.e. the initial object in the category of such modules.

Lemma 6.2.4. Let $M$ be a left module over a truss $T$. Then $M$ is a free $T$-module if and only if $M$ has a basis. 
Proof. If $M$ has a basis $B$, then $\underset{x \in B}{\boxplus} \sigma_{x}$ is an epimorphism. Since all elements of $\mathcal{T}^{B}$ have finite length, i.e. every element belongs to $\underset{S}{\boxplus} T$ for a finite subset $S$ of $B$, and for all such subsets $\underset{x \in S}{\boxplus} \sigma_{x}$ is one-to-one, then so is $\underset{x \in B}{\boxplus} \sigma_{x}$. Thus $\underset{x \in B}{\boxplus} \sigma_{x}$ is an isomorphism, and hence $M$ is free.

In the converse direction, since $M$ is free there exist a set $X$ and a left $T$-module isomorphism $\Theta: \underset{x \in X}{\boxplus} T x \longrightarrow M$. For all $x \in X$, let $e_{x}=\Theta(x) \in M$, and let $B=\left\{e_{x} \mid x \in X\right\}$. Since $\Theta$ is a homomorphism of $T$-modules, for all $t \in T$,

$$
\Theta(t x)=t \Theta(x)=t \cdot e_{x}=\sigma_{e_{x}}(t)
$$

Since $X$ is isomorphic to $B$, by the universality of direct sums there is a $T$-module isomorphism $\varphi: \underset{B}{\boxplus} T \longrightarrow \mathcal{T}^{X}$ and thus we obtain the following commutative diagrams, for all $e_{x} \in B$,

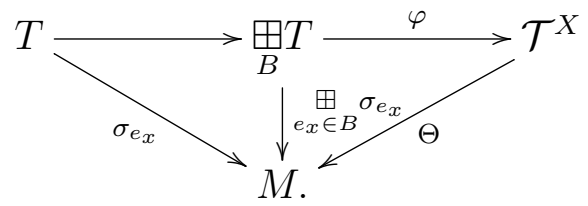

Thus $\underset{e_{x} \in B}{\boxplus_{e_{x}}} \sigma_{e}$ is an isomorphism, and hence $B$ generates $M$. Since $\underset{e_{x} \in B}{\boxplus} \sigma_{e_{x}}$ is a monomorphism on $\underset{B}{\boxplus} T$, it is a monomorphism on each finitely generated submodule of $\boxplus_{B}^{\boxplus} T$, in particular $\underset{e_{x} \in S}{\boxplus} \sigma_{e_{x}}=\left.\underset{e_{x} \in B}{\boxplus} \sigma_{e_{x}}\right|_{S}$ is a monomorphism for all finite subsets $S$ of $B$. Hence $B$ is a basis for $M$.

The forthcoming Lemma 6.2.6 clarifies when a module over the truss associated to a ring is a module over this ring. Before we state this lemma, however, we make an observation about a striking difference between free modules over a ring and free modules over the associated truss. We note, in particular, that the functor $\mathrm{T}$ does not preserve freeness.

Example 6.2.5. Let us consider the module $\mathrm{T}\left(\mathbb{Z}_{n} \oplus \mathbb{Z}_{n}\right)$ over $\mathrm{T}\left(\mathbb{Z}_{n}\right)$, for any $n>1$. Suppose that $\mathrm{T}\left(\mathbb{Z}_{n} \oplus \mathbb{Z}_{n}\right)$ is a free module, i.e. that it is isomorphic to a direct sum of $k$-copies of $\mathrm{T}\left(\mathbb{Z}_{n}\right)$. By Proposition 1.4.4, if $k>1$ then such a direct sum would be an infinite set, so it cannot be isomorphic to a module built on a finite set. Thus $k=1$, 
and simple element counting forces $n=n^{2}$, which contradicts the assumption that $n>1$. Thus $\mathrm{T}\left(\mathbb{Z}_{n} \oplus \mathbb{Z}_{n}\right)$ over $\mathrm{T}\left(\mathbb{Z}_{n}\right)$ is not free, despite that fact that $\mathbb{Z}_{n} \oplus \mathbb{Z}_{n}$ is a free $\mathbb{Z}_{n}$-module.

Recall that an element $e$ of a $T$-module $M$ is called an absorber, if $t \cdot e=e$, for all $t \in T$. The set of all absorbers of $M$ is denoted by $\operatorname{Abs}(M)$.

Lemma 6.2.6. Let $T$ be a (unital) truss and $R$ a (unital) ring.

(1) The assignment:

$$
\text { Abs }: T-\bmod \longrightarrow T-\bmod , \quad M \longmapsto \operatorname{Abs}(M), \quad \varphi \longmapsto \varphi
$$

is a functor.

(2) Let $M$ be a left module over $T(R)$. Then:

(i) $\operatorname{Abs}(M)=\{0 \cdot m \mid m \in M\}$;

(ii) $M=\mathrm{T}(N)$ for some module of $N$ if and only if $\operatorname{Abs}(M)$ is a singleton set.

(3) Let $N, N^{\prime}$ be left $R$-modules. Then $N$ is isomorphic to $N^{\prime}$ if and only if $\mathrm{T}(N)$ is isomorphic to $\mathrm{T}\left(N^{\prime}\right)$ as $\mathrm{T}(R)$-modules.

(4) Let $M$ be a (unital) $\mathrm{T}(R)$-module. Then $\mathrm{G}(M / \operatorname{Abs}(M) ; \operatorname{Abs}(M))$ is a (unital) $R$-module. We denote this $R$-module by $M_{\mathrm{Abs}}$.

(5) The assignment

$$
\begin{aligned}
(-)_{\mathrm{Abs}}: \mathrm{T}(R)-\bmod \longrightarrow R \text {-mod } & \\
M & \longmapsto M_{\mathrm{Abs}}, \quad\left(\varphi: M \rightarrow M^{\prime}\right) \longmapsto\left(\varphi_{\mathrm{Abs}}: \bar{m} \mapsto \overline{\varphi(m)}\right),
\end{aligned}
$$

is a functor such that, for all $R$-modules $N, T(N)_{\mathrm{Abs}} \cong N$.

(6) The functor $(-)_{\mathrm{Abs}}$ is the left adjoint to the functor $\mathrm{T}$.

(7) The functor $(-)_{\text {Abs }}$ preserves monomorphisms.

Proof. (1) The distributive law over the heap operation in a $T$-module $M$ ensures that $\operatorname{Abs}(M)$ is a sub-heap of $M$. That $\operatorname{Abs}(M)$ is closed under the action follows 
immediately form the definition of an absorber. Since morphisms of $T$-modules preserve the $T$-action they also map absorbers into absorbers.

(2) (i) Since $r 0=0$ in $\mathrm{T}(R)$, all elements listed are absorbers. If $e$ is an absorber, then, by the absorption property $0 \cdot e=e$.

(2) (ii) If $M=\mathrm{T}(N)$, then, by distributive laws for modules over rings 0 is an absorber in $M$ and $0 \cdot m=0 \in M$, for all $m \in M$, which implies that 0 is the unique absorber of $M$. In converse direction, by (i) we know that the unique absorber is $e=0 \cdot m$. Then one easily checks that $\mathrm{G}(M ; e)$ with the original action of $\mathrm{T}(R)$ on $M$ is a left $R$-module.

(3) Since $\mathrm{T}$ is a functor, if $N \cong N^{\prime}$, then $\mathrm{T}(N) \cong \mathrm{T}\left(N^{\prime}\right)$. Conversely, since, by statement (2) both $\mathrm{T}(N)$ and $\mathrm{T}\left(N^{\prime}\right)$ have unique absorbers (they are neutral elements for addition), and a morphism of modules over a truss maps absorbers into absorbers (cf. statement (1)), any morphism of $\mathrm{T}(R)$-modules $\mathrm{T}(N) \longrightarrow \mathrm{T}\left(N^{\prime}\right)$ is automatically a morphism of Abelian groups and hence $R$-modules.

(4) Since $\operatorname{Abs}(M)$ is a submodule of $M$ by assertion (1), $M / \operatorname{Abs}(M)$ is a $\mathrm{T}(R)$ module with an absorber $\operatorname{Abs}(M)$. There are no other absorbers in $M / \operatorname{Abs}(M)$, since $M / \operatorname{Abs}(M)$ is a module of $\mathrm{T}(R)$, by statement (2)(i) all its absorbers have the form $0 \cdot \bar{m}=\overline{0 \cdot m}=\operatorname{Abs}(M)$. Thus, by statement (2)(ii), $\mathrm{G}(M / \operatorname{Abs}(M) ; \operatorname{Abs}(M))$ is a left $R$-module. The unitality condition is provided by the unitality of the $\mathrm{T}(R)$-module $M$.

(5) The function $\varphi_{\text {Abs }}$ is well-defined by statement (1), as $\varphi$ maps absorbers to absorbers. By the same arguments as in the proof of statement (3) $\varphi_{\text {Abs }}$ is a homomorphism of $R$-modules. Since $\operatorname{Abs}(\mathrm{T}(N))=\{0\}$. The elements of $\mathrm{T}(N) /\{0\}$ are all singleton subsets of $N, \mathrm{~T}(N) /\{0\}=\{\{n\} \mid n \in N\}$, and the stated isomorphism is simply $\{n\} \longmapsto n$.

(6) Let $N$ be a left $R$-module and $M$ a left $\mathrm{T}(R)$-module, and consider the maps:

$$
\begin{aligned}
& \Theta_{M, N}: \operatorname{Hom}_{R}\left(M_{\mathrm{Abs}}, N\right) \longrightarrow \operatorname{Hom}_{\mathrm{T}(R)}(M, \mathrm{~T}(N)), \quad \varphi \longmapsto[m \mapsto \varphi(\bar{m})], \\
& \Theta_{M, N}^{-1}: \operatorname{Hom}_{\mathrm{T}(R)}(M, \mathrm{~T}(N)) \longrightarrow \operatorname{Hom}_{R}\left(M_{\mathrm{Abs}}, N\right), \quad \psi \longmapsto[\bar{m} \mapsto \psi(m)],
\end{aligned}
$$

that are clearly mutual inverses. While $\Theta_{M, N}$ is obviously well-defined, we need to estab- 
lish whether the definition of $\Theta_{M, N}^{-1}$ does not depend on the choice of the representative. Suppose that $m^{\prime}$ and $m$ belong to the same class. In view of the description on $\operatorname{Abs}(M)$ in $(2)(\mathrm{i})$ this means that there exist $v, w \in M$ such that $\left[m, m^{\prime}, 0 v\right]=0 w$. Applying $\psi$ to this equality and using the fact that $\psi$ is a homomorphism of $\mathrm{T}(R)$-modules we find that $\left[\psi(m), \psi\left(m^{\prime}\right), 0 \psi(v)\right]=0 \psi(w)$. Both $0 \psi(v)$ and $0 \psi(w)$ are absorbers in $\mathrm{T}(N)$, but, by (2)(ii) there is exactly one absorber in $\mathrm{T}(N)$, so $0 \psi(v)=0 \psi(w)$, and we conclude that $\psi(m)=\psi\left(m^{\prime}\right)$. Thus the definition of $\Theta_{M, N}^{-1}$ does not depend on the choice of the representative in the class of $m$. Checking the naturality of $\Theta_{M, N}$ is straightforward.

(7) Let $\varphi: M \rightarrow M^{\prime}$ be a monomorphism of $\mathrm{T}(R)$-modules. Assume that $\varphi_{\mathrm{Abs}}(\bar{a})=$ $\varphi_{\mathrm{Abs}}(\bar{b})$, then $\varphi(a) \sim_{\mathrm{Abs}} \varphi(b)$, and there exist $w, z \in \operatorname{Abs}(M)$ such that

$$
\varphi(z)=[\varphi(a), \varphi(b), \varphi(w)]=\varphi([a, b, w])
$$

Since $\varphi$ is one-to-one, $[a, b, w]=z \in \operatorname{Abs}(M)$, i.e., $\bar{a}=\bar{b}$. Therefore, $\varphi_{\text {Abs }}$ is a monomorphism, as required.

With the help of Lemma 6.2.6 we can prove the main result of this section, which explains the interplay between the freeness and the functor $\mathrm{T}$.

Theorem 6.2.7. Let $R$ be a unital ring.

(1) For a left $R$-module $N, \mathrm{~T}(N)$ is a free $\mathrm{T}(R)$-module if and only if $N \cong R$.

(2) If $M$ is a free module over $\mathrm{T}(R)$, then $M_{\mathrm{Abs}}$ is a free $R$-module.

Proof. (1) If $N \cong R$, then $\mathrm{T}(N) \cong \mathrm{T}(R)$ by Lemma 6.2.6 (3) (or simply by the fact that $\mathrm{T}$ is a functor). In the opposite direction, assume that there exists a set $X$ such that $\mathrm{T}(N) \cong \underset{x \in X}{\boxplus} \mathrm{T}(R) x$. By Lemma 6.2.6 (2)(ii), $\underset{x \in X}{\boxplus} \mathrm{T}(R) x$ must have exactly one absorber. This is the case when $X$ is a singleton set, which yields the isomorphism $N \cong R$ by Lemma 6.2.6 (3). If $X$ has more than one element, then there exist $x, y \in X$ such that $x \neq y$ and thus $0 x \neq 0 y \in \underset{x \in X}{\boxplus} \mathrm{T}(R) x$ are different absorbers, which contradicts statement (2)(ii) in Lemma 6.2.6. 
(2) Assume that $M \cong \underset{x \in X}{\boxplus} \mathrm{T}(R) x$, for some set $X$. By Lemma 6.2.6 $(6),(-)_{\text {Abs }}$ has a right adjoint and thus it preserves coproducts, so that

$$
M_{\mathrm{Abs}} \cong(\underset{x \in X}{\boxplus} \mathrm{T}(R) x)_{\mathrm{Abs}} \cong \bigoplus_{x \in X}(\mathrm{~T}(R) x)_{\mathrm{Abs}} \cong \bigoplus_{x \in X} R x
$$

where the last isomorphism follows by Lemma 6.2.6 (5). Therefore, $M_{\mathrm{Abs}}$ is a free $R$-module as stated.

Although Theorem 6.2.7 states that the functor $(-)_{\text {Abs }}$ preserves freeness the proof neither gives an insight into the process of obtaining the free $R$-module nor does it explain fully the idea behind the definition of a basis in Definition 6.2.2. Let us discuss this matter further in the finitely generated case. Let $X=\left\{x_{1}, \ldots, x_{n}\right\}$ be such that $M \cong \underset{x \in X}{\boxplus} \mathrm{T}(R) x$. First we describe the submodule $\operatorname{Abs}(M)$, freely identifying $M$ with the direct sum of $n$ copies of $T$. By Lemma 6.2.6 (2)(i), $\operatorname{Abs}(M)=\{0 \cdot m \mid m \in M\}$. Since $\mathrm{T}(R) x_{i}=$

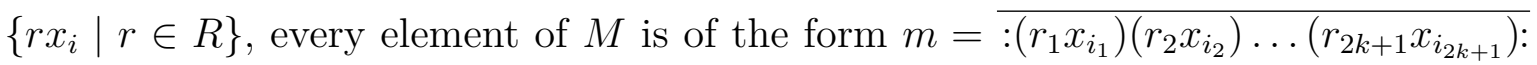
and hence $0 \cdot m=\overline{:\left(0 x_{i_{1}}\right)\left(0 x_{i_{2}}\right) \ldots\left(0 x_{i_{2 k+1}}\right):}$. Therefore $\operatorname{Abs}(M)$ is the submodule of $M$, or, more precisely $\operatorname{Abs}(\underset{x \in X}{\boxplus} \mathrm{T}(R) x)$, is a submodule of $\underset{x \in X}{\boxplus} \mathrm{T}(R) x$ generated by $\left\{0 x_{1}, \ldots, 0 x_{n}\right\}$ as a heap. Choosing the $0 x_{i}$ as special elements in $\mathrm{T}(R) x_{i}$ as in (the multi-heap versions of) Proposition 1.4.1, $\operatorname{Abs}(\underset{x \in X}{\boxplus} \mathrm{T}(R) x)$ is simply the sub-heap of tails, i.e.

$$
\operatorname{Abs}(M) \cong \operatorname{Abs}(\underset{x \in X}{\boxplus} \mathrm{T}(R) x) \cong \mathcal{H}\left(\left\{0 x_{1}\right\}\right) \boxplus \mathcal{H}\left(\left\{0 x_{2}\right\}\right) \boxplus \ldots \boxplus \mathcal{H}\left(\left\{0 x_{n}\right\}\right) \cong \mathrm{H}\left(\mathbb{Z}^{n-1}\right)
$$

see Corollary 1.4.6. By (the multi-heap extension of Proposition 1.4.4

$$
M \cong{\underset{x \in X}{\boxplus}}_{\operatorname{m}} \mathrm{T}(R) x \cong \mathrm{H}\left(\bigoplus_{i=1}^{n} \mathrm{G}\left(\mathrm{T}(R) x_{i} ; 0 x_{i}\right) \oplus \mathbb{Z}^{n-1}\right) \cong \mathrm{H}\left(R^{n} \oplus \mathbb{Z}^{n-1}\right) .
$$

Since the $\mathbb{Z}^{n-1}$-part arises from tails made of the absorbers $0 x_{i}$, the action of $T(R)$ on this part is trivial, i.e. the $\mathrm{T}(R)$ action on $M$ transfers to

$$
r \cdot\left(r_{1}, r_{2}, \ldots, r_{n}, k_{1}, \ldots, k_{n-1}\right)=\left(r r_{1}, r r_{2}, \ldots, r r_{n}, k_{1}, \ldots, k_{n-1}\right), \quad r, r_{i} \in R, k_{i} \in \mathbb{Z}
$$

Putting all this together yields an isomorphism of $R$-modules,

$$
M_{\mathrm{Abs}}=\mathrm{G}(M / \operatorname{Abs}(M) ; \operatorname{Abs}(M)) \cong\left(R^{n} \oplus \mathbb{Z}^{n-1}\right) / \mathbb{Z}^{n-1} \cong R^{n}
$$


where the first isomorphism follows by Corollary 1.2.15, so that $M_{\mathrm{Abs}}$ is a free module.

Now, assume that $M$ is a free rank $n$ module over $\mathrm{T}(R)$ with a basis $B$. To prove that $B$ is a basis for $M_{\mathrm{Abs}}$ observe that, for all $S \subset B,\left(\underset{s \in S}{\boxplus} \sigma_{s}\right)_{\mathrm{Abs}}$ is a monomorphism of $R$-modules(see Lemma 6.2.6 (7)), and by the discussion following Lemma 6.2.3. appropriate intersections of $\left(\underset{s \in S}{\boxplus} \sigma_{s}\right)$ Abs $(\underset{S}{\boxplus} \mathrm{T}(R))_{\mathrm{Abs}}$ are no longer empty; they are now the initial object of $R$-mod, i.e. $\{0\}$. Firstly, since $B$ spans $M$, then it also spans $M_{\mathrm{Abs}}$. Therefore, it is enough to show that the set $B$ is linearly independent in $M_{\mathrm{Abs}}$. Suppose to the contrary that $B$ is linearly dependent, so that there exist $r_{i} \in R$ such that

$$
r_{1} b_{1}+\ldots+r_{n} b_{n}=0
$$

for $b_{i} \in B$, and $r_{n} \neq 0$. This implies that $r_{1} b_{1}+\ldots+r_{n-1} b_{n-1}=-r_{n} b_{n}$. Furthermore

$$
-r_{n} b_{n} \in\left(\stackrel{n}{\boxplus=1}_{i=1}^{\boxplus-1} \sigma_{b_{i}}\right) \text { Abs }\left({ }_{i=1}^{n-1} \mathrm{~T}(R)\right)_{A b s} \cap\left(\sigma_{b_{n}}\right)_{\mathrm{Abs}}\left((\mathrm{T}(R))_{\mathrm{Abs}}\right)=\{0\} .
$$

Therefore, $-r_{n} b_{n}=0$ and $\left(\sigma_{b_{n}}\right)_{\mathrm{Abs}}(0)=\left(\sigma_{b_{n}}\right)_{\mathrm{Abs}}\left(-r_{n}\right)$, and since $\left(\sigma_{b_{n}}\right)_{\mathrm{Abs}}$ is a monomorphism, $r_{n}=0$. Now by recursion for all $i=1, \ldots, n, r_{i}=0$, and we arrive at a contradiction with the assumption that $B$ is a linearly dependent set. Therefore, $B$ is a basis for $M_{\mathrm{Abs}}$. To sum up, at least in the case of the truss associated to a ring, Definition 6.2 .2 of a free set is justified by the linear independence of its elements in the associated module over a ring.

\subsection{Tensor product}

In this section, we will construct and investigate a tensor product of modules over trusses. Now $T$ is an arbitrary truss, not necessarily unital. Let us start with the following definition of bilinear maps.

Definition 6.3.1. Let $H, M, N$ be Abelian heaps. A function $\varphi: M \times N \longrightarrow H$ is said to be bilinear if, for all $m, m^{\prime}, m^{\prime \prime} \in M$ and $n, n^{\prime}, n^{\prime \prime} \in N$,

$$
\begin{aligned}
& \varphi\left(\left[m, m^{\prime}, m^{\prime \prime}\right], n\right)=\left[\varphi(m, n), \varphi\left(m^{\prime}, n\right), \varphi\left(m^{\prime \prime}, n\right)\right] \\
& \varphi\left(m,\left[n, n^{\prime}, n^{\prime \prime}\right]\right)=\left[\varphi(m, n), \varphi\left(m, n^{\prime}\right), \varphi\left(m, n^{\prime \prime}\right)\right] .
\end{aligned}
$$


In addition, if $T$ is a truss, $M$ is a right $T$-module and $N$ is a left $T$-module, then $\varphi$ is said to be $T$-balanced if, for all $m \in M, n \in N, t \in T$,

$$
\varphi(m \cdot t, n)=\varphi(m, t \cdot n) .
$$

Remark 6.3.2. We note in passing that, due to the Mal'cev conditions, any heap homomorphism $\varphi: M \times N \longrightarrow H$ satisfies conditions 6.3.1a 6.3.1b in Definition 6.3.1 (but, of course, a function satisfying 6.3.1a) 6.3.1b) needs not be a homomorphism of heaps).

The definition of the tensor product of modules over a truss is given by the following universal property, reminiscent of that for the tensor product of modules over a ring.

Definition 6.3.3. Let $M$ be a right $T$-module and $N$ be a left $T$-module. Then a tensor product (of $M$ and $N$ over $T$ ) is a pair $\left(M \otimes_{T} N, \varphi\right)$ consisting of an Abelian heap $M \otimes_{T} N$ and a $T$-balanced bilinear map $\varphi: M \times N \longrightarrow M \otimes_{T} N$ such that for any heap $H$ and any $T$-balanced bilinear map $f: M \times N \longrightarrow H$ there exists a unique heap morphism $\hat{f}$ rendering commutative the following diagram

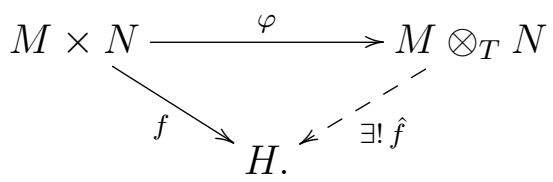

As for tensor products of modules over rings, if a tensor product of $M$ and $N$ over $T$ exists, then it is unique up to a unique isomorphism. Thus, we will speak about the tensor product $M \otimes_{T} N$, often omitting the structure map $\varphi$ as well.

Since any Abelian heap is a unital module over the terminal truss $\{1\}$ in a unique way, one can consider tensor product of heaps. In this case the balancing condition 6.3.2a is tautologically satisfied. The tensor product of heaps $M$ and $N$ viewed as unital $\{1\}$-modules is denoted by $M \otimes N$. Observe that, differently from what happens for modules over a ring, the fact that $1_{M \times N}$ is bilinear entails that there exists a unique morphism of heaps $\sigma: M \otimes N \rightarrow M \times N$ such that $\sigma \circ \varphi=1_{M \times N}$ (see Remark 6.3.2).

Next we give an explicit construction of tensor products, thus establishing their existence. 
Theorem 6.3.4. Tensor product of T-modules exists.

Proof. Let $M$ be a right $T$-module, $N$ be a left $T$-module and let us consider the free Abelian heap $\mathcal{A}(M \times N)$. Choose an arbitrary element $e=\left(e_{1}, e_{2}\right)$ of the free heap $\mathcal{A}(M \times N)$ and let $S_{T}(e)$ be the sub-heap of $\mathcal{A}(M \times N)$ generated by elements of the form:

$$
\begin{gathered}
{\left[\left(\left[m, m^{\prime}, m^{\prime \prime}\right]_{M}, n\right),\left[(m, n),\left(m^{\prime}, n\right),\left(m^{\prime \prime}, n\right)\right]_{\mathcal{A}}, e\right]_{\mathcal{A}},} \\
{\left[\left(m,\left[n, n^{\prime}, n^{\prime \prime}\right]_{N}\right),\left[(m, n),\left(m, n^{\prime}\right),\left(m, n^{\prime \prime}\right)\right]_{\mathcal{A}}, e\right]_{\mathcal{A}}} \\
{[(m \cdot t, n),(m, t \cdot n), e]_{\mathcal{A}},}
\end{gathered}
$$

for all $m, m^{\prime}, m^{\prime \prime} \in M, n, n^{\prime}, n^{\prime \prime} \in N$ and $t \in T$. Note that the transposition rule 1.1.2 together with the idempotent property of a heap operation imply that every element of $S_{T}(e)$ has the form $[a, b, e]$, where $a, b \in \mathcal{A}(M \times N)$. Also note that $e \in S_{T}(e)$. For an Abelian heap $H$, consider a $T$-balanced bilinear map $f: M \times N \longrightarrow H$. By treating $f$ as a function and by using the universal property of the free heap, we can construct the following commutative diagram:

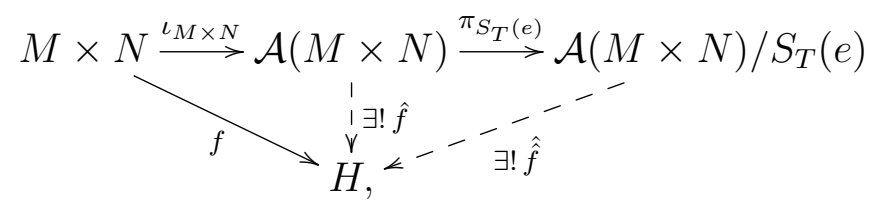

where $\iota_{M \times N}$ is the canonical monomorphism and $\pi_{S_{T}(e)}$ is the canonical epimorphism. The left triangle is given by the free heap property. The existence of the map $\hat{\hat{f}}$ is guaranteed provided that $\hat{f}$ respects the sub-heap relation $\sim_{S_{T}(e)}$. By using the definition of $\hat{f}$ and that $f$ is a $T$-balanced bilinear map, we find

$$
\begin{aligned}
\hat{f}\left(\left[\left(m,\left[n, n^{\prime}, n^{\prime \prime}\right]_{N}\right),\left[(m, n),\left(m, n^{\prime}\right),\left(m, n^{\prime \prime}\right)\right]_{\mathcal{A}}, e\right]_{\mathcal{A}}\right) & \\
= & {\left[f\left(m,\left[n, n^{\prime}, n^{\prime \prime}\right]_{N}\right),\left[f(m, n), f\left(m, n^{\prime}\right), f\left(m, n^{\prime \prime}\right)\right]_{H}, f(e)\right]_{H} } \\
\frac{6.3 .1 \mathrm{~b}}{-} & {\left[f\left(m,\left[n, n^{\prime}, n^{\prime \prime}\right]_{N}\right), f\left(m,\left[n, n^{\prime}, n^{\prime \prime}\right]_{N}\right), f(e)\right]_{H}=f(e)=\hat{f}(e) . }
\end{aligned}
$$

By symmetric arguments,

$$
\hat{f}\left(\left[\left(\left[m, m^{\prime}, m^{\prime \prime}\right]_{M}, n\right),\left[(m, n),\left(m^{\prime}, n\right),\left(m^{\prime \prime}, n\right)\right]_{\mathcal{A}}, e\right]_{\mathcal{A}}\right)=\hat{f}(e) .
$$


Finally,

$$
\begin{aligned}
\hat{f}\left([(m \cdot t, n),(m, t \cdot n), e]_{\mathcal{A}}\right) & =[f(m \cdot t, n), f(m, t \cdot n), f(e)]_{H} \\
& =[f(m \cdot t, n), f(m \cdot t, n), f(e)]=f(e)=\hat{f}(e) .
\end{aligned}
$$

This means that $S_{T}(e) \subset \operatorname{ker}_{\hat{f}(e)}(\hat{f})$ and therefore, in view of Lemma 1.2.17, $\hat{f}$ respects the sub-heap relation $\sim_{S_{T}(e)}$ as required. Consequently, the heap homomorphism $\hat{\hat{f}}$ exists.

Define:

$$
\varphi:=\left(\pi_{S_{T}(e)} \circ \iota_{M \times N}\right): M \times N \longrightarrow \mathcal{A}(M \times N) / S_{T}(e), \quad(m, n) \longmapsto \overline{(m, n)} .
$$

Since $e \in S_{T}(e)$, by definition of $\sim_{S_{T}(e)}$ and of $[-,-,-]$ on $\mathcal{A}(M \times N) / S_{T}(e)$,

$$
\begin{gathered}
\overline{\left(\left[m, m^{\prime}, m^{\prime \prime}\right]_{M}, n\right)}=\overline{\left[(m, n),\left(m^{\prime}, n\right),\left(m^{\prime \prime}, n\right)\right]_{\mathcal{A}}}=\left[\overline{(m, n)}, \overline{\left(m^{\prime}, n\right)}, \overline{\left(m^{\prime \prime}, n\right)}\right] \\
\overline{\left(m,\left[n, n^{\prime}, n^{\prime \prime}\right]_{N}\right)}=\overline{\left[(m, n),\left(m, n^{\prime}\right),\left(m, n^{\prime \prime}\right)\right]_{\mathcal{A}}}=\left[\overline{(m, n)}, \overline{\left(m, n^{\prime}\right)}, \overline{\left(m, n^{\prime \prime}\right)}\right] \\
\text { and } \quad \overline{(m \cdot t, n)}=\overline{(m, t \cdot n)}
\end{gathered}
$$

hold in $\mathcal{A}(M \times N) / S_{T}(e)$, that is to say, $\varphi$ is a $T$-balanced bilinear map. It remains to prove that the map $\hat{\hat{f}}$ constructed in diagram (6.3.4) is a unique homomorphism such that $f=\hat{\hat{f}} \circ \pi_{S_{T}(e)} \circ \iota_{M \times N}$. Suppose that there exists another homomorphism of heaps $h: \mathcal{A}(M \times N) / S_{T}(e) \longrightarrow H$ such that $f=h \circ \pi_{S_{T}(e)} \circ \iota_{M \times N}$. Then

$$
h \circ \pi_{S_{T}(e)} \circ \iota_{M \times N}=\hat{\hat{f}} \circ \pi_{S_{T}(e)} \circ \iota_{M \times N},
$$

and, since both $h \circ \pi_{S_{T}(e)}$ and $\hat{\hat{f}} \circ \pi_{S_{T}(e)}$ are homomorphisms of heaps, the universal property of the free (Abelian) heap implies that

$$
h \circ \pi_{S_{T}(e)}=\hat{\hat{f}} \circ \pi_{S_{T}(e)} .
$$

Since $\pi_{S_{T}(e)}$ is an epimorphism, it follows that $h=\hat{\hat{f}}$ and the uniqueness is established. Therefore, the pair $\left(\mathcal{A}(M \times N) / S_{T}(e), \varphi\right)$ is the tensor product of $M$ and $N$.

We note in passing that, up to isomorphism, the construction of the tensor product does not depend on the chosen element $e$. This independence can be seen as a consequence of the universal property of tensor products, or it can be observed directly by employing the swap automorphism (1.1.4. 
Following the ring-theoretic conventions we define, for all $m \in M$ and $n \in N$,

$$
m \otimes n:=\overline{(m, n)}=\left(\pi_{S_{T}(e)} \circ \iota_{M \times N}\right)(m, n) \in M \otimes_{T} N
$$

and we refer to each of $m \otimes n$ as to a simple tensor. As a rule, we do not decorate $\otimes$ with a subscript $T$, but occasionally it might be useful to indicate an element $e$ chosen in the definition of $S_{T}(e)$, in which case we write $m \otimes_{e} n$. With this terminology and notation at hand, $M \otimes_{T} N$ can be understood as an Abelian heap freely generated by simple tensors subject to relations:

$$
\begin{gathered}
{\left[m, m^{\prime}, m^{\prime \prime}\right] \otimes n=\left[m \otimes n, m^{\prime} \otimes n, m^{\prime \prime} \otimes n\right], \quad \text { for all } m, m^{\prime}, m^{\prime \prime} \in M, n \in N,} \\
m \otimes\left[n, n^{\prime}, n^{\prime \prime}\right]=\left[m \otimes n, m \otimes n^{\prime}, m \otimes n^{\prime \prime}\right], \quad \text { for all } m \in M, n, n^{\prime}, n^{\prime \prime} \in N, \\
m \cdot t \otimes n=m \otimes t \cdot n, \quad \text { for all } m \in M, n \in N, t \in T
\end{gathered}
$$

Definition 6.3.5. An opposite truss $T^{o p}$ of a truss $T$ is a triple $(T,[-,-,-], \bullet)$ such that $[-,-,-]$ is the ternary operation of $T$, and $\bullet: T \otimes T \rightarrow T$ is given for all $a, b \in T$ by

$$
a \bullet b=b a
$$

where $b a$ is a product in $T$.

Remark 6.3.6. $T^{o p}$ is a truss.

The following technical result will be of significant importance for Morita theory in chapter 7 .

Proposition 6.3.7. Let $T$ be a truss and $T_{u}$ be its unital extension. Then for every right $T$-module $M, M \otimes_{T} T_{u} \cong M \cong \operatorname{Hom}_{T}\left(T_{u}, M\right)$ as right $T$-modules, where $T_{u}$ has the T-T-bimodule structure induced by the truss homomorphism $\iota_{T}: T \rightarrow T_{u}$. Moreover, for $M$ a right $T$-module and $N$ a left $T$-module

$$
m \cdot z \otimes_{T} n=m \otimes_{T} z \cdot n
$$

for all $m \in M, n \in N, z \in T_{u}$. 
Proof. Consider the assignment

$$
\alpha: M \longrightarrow M \otimes_{T} T_{u}, \quad m \longmapsto m \otimes_{T} 1 .
$$

This is a heap homomorphism in view of 6.3.6a and it is $T$-linear because for all $t \in T$,

$$
\alpha(m \cdot t)=m \cdot t \otimes_{T} 1 \stackrel{\sqrt[6.3 .6 \mathrm{c}]{-}}{-} m \otimes_{T} \iota_{T}(t) \cdot 1 \stackrel{5.1 .7}{-} m \otimes_{T} 1 \cdot \iota_{T}(t)=\left(m \otimes_{T} 1\right) \cdot t .
$$

The other way around, recall that the underlying Abelian heap of $\mathrm{E}_{1}(M)$ is $M$ itself, which now is considered as a unital $T_{u}$-module via the bilinear morphism $\varrho: M \times T_{u} \longrightarrow$ $M$ uniquely determined by

$$
\left\{\begin{array}{l}
(m, t) \longmapsto m \cdot t, \quad t \in T, \\
(m, 1) \longmapsto m .
\end{array}\right.
$$

The associativity of the $T$-action entails that $\varrho$ is also $T$-balanced, whence it factors through the tensor product over $T$ giving

$$
\beta: M \otimes_{T} T_{u} \longrightarrow M
$$

A straightforward check shows that $\alpha$ and $\beta$ are inverses of each other. Concerning the second isomorphism, consider the right $T$-linear morphism

$$
\operatorname{Hom}_{T}\left(T_{u}, M\right) \longrightarrow M, \quad f \longmapsto f(1),
$$

and the assignment $M \longrightarrow \operatorname{Hom}_{T}\left(T_{u}, M\right)$, sending every $m \in M$ to the right $T$-linear morphism uniquely determined by

$$
\left\{\begin{array}{l}
t \longmapsto m \cdot t, \quad t \in T, \\
1 \longmapsto m .
\end{array}\right.
$$

Again, a straightforward check shows that they are inverses of each other. To prove the last assertion, recall that an element $z$ in $T_{u}$ is of the form $\left[a_{1}, \ldots, a_{s}\right]$, where $a_{i} \in T \sqcup\{1\}$, for all $i=1, \ldots, s$ and $s$ odd. Therefore,

$$
\begin{aligned}
m \cdot z \otimes_{T} n & =m \cdot\left[a_{1}, \ldots, a_{s}\right] \otimes_{T} n=\left[m \cdot a_{1} \otimes_{T} n, \ldots, m \cdot a_{s} \otimes_{T} n\right] \\
& \stackrel{(\bullet)}{=}\left[m \otimes_{T} a_{1} \cdot n, \ldots, m \otimes_{T} a_{s} \cdot n\right]=m \otimes_{T} z \cdot n,
\end{aligned}
$$

where (•) follows from the fact that either $m \cdot a_{i} \otimes_{T} n=m \cdot t \otimes_{T} n=m \otimes_{T} t \cdot n$ (if $a_{i} \in T$ ) or $m \cdot a_{i} \otimes_{T} n=m \otimes_{T} n=m \otimes_{T} a_{i} \cdot n$ (if $a_{i}=1 \in\{1\}$ ). 
In parallel to the ring-theoretic tensor product, tensoring with a fixed bimodule defines a functor between categories of modules over trusses.

Proposition 6.3.8. Let $T$ and $S$ be trusses.

1. If $M$ is a right $T$-module and $N$ is a $T$-S-bimodule, then $M \otimes_{T} N$ is a right $S$-module with the action

$$
\left(M \otimes_{T} N\right) \times S \longrightarrow M \otimes_{T} N, \quad(m \otimes n, s) \longmapsto m \otimes n \cdot s .
$$

If $S$ admits a unit and $N$ is unital, then $M \otimes_{T} N$ is unital as well. Symmetrically, if $M$ is an $S$-T-bimodule (unital over $S$ ) and $N$ is a left T-module, then $M \otimes_{T} N$ is a (unital) left $S$-module.

2. Let $N$ be a T-S-bimodule and let $\varphi: M \longrightarrow M^{\prime}$ be a homomorphism of right $T$-modules. Then the map $\varphi \otimes N$ defined on simple tensors as

$$
\varphi \otimes N: M \otimes_{T} N \longmapsto M^{\prime} \otimes_{T} N, \quad m \otimes n \longmapsto \varphi(m) \otimes n,
$$

extends uniquely to a homomorphism of right $S$-modules. Symmetrically, if $M$ is an $S$-T bimodule, then any left T-module homomorphism $\varphi: N \longrightarrow N^{\prime}$ gives rise to a left S-module homomorphism,

$$
M \otimes \varphi: M \otimes_{T} N \longmapsto M \otimes_{T} N^{\prime}, \quad m \otimes n \longmapsto m \otimes \varphi(n) .
$$

3. The constructions in items (1) and (2) yield functors $-\otimes_{T} N: \bmod -T \longrightarrow \bmod -S$ and $M \otimes_{T}-: T$-mod $\longrightarrow S$-mod. Furthermore, if $S$ admits a unit and $M, N$ are unital (over $S$ ), then they yield functors $-\otimes_{T} N: \bmod -T \longrightarrow \bmod -S_{1}$ and $M \otimes_{T}-: T-\bmod \longrightarrow S_{1}-\bmod$.

Proof. 1. Since $N$ is a right $S$-module, for every $s \in S$ we can consider the assignment

$$
\rho_{s}: M \times N \longrightarrow M \otimes_{T} N, \quad(m, n) \longmapsto m \otimes n \cdot s .
$$


It satisfies

$$
\begin{aligned}
\rho_{s}\left(\left(\left[m, m,,^{\prime}, m^{\prime \prime}\right], n\right)\right) & =\left[m, m,{ }^{\prime}, m^{\prime \prime}\right] \otimes n \cdot s \stackrel{6.3 .6 \mathrm{a}}{=}\left[m \otimes n \cdot s, m^{\prime} \otimes n \cdot s, m^{\prime \prime} \otimes n \cdot s\right] \\
& =\left[\rho_{s}(m, n), \rho_{s}\left(m^{\prime}, n\right), \rho_{s}\left(m^{\prime \prime}, n\right)\right], \\
\rho_{s}\left(m,\left[n, n^{\prime}, n^{\prime \prime}\right]\right) & =m \otimes\left[n, n^{\prime}, n^{\prime \prime}\right] \cdot s=m \otimes\left[n \cdot s, n^{\prime} \cdot s, n^{\prime \prime} \cdot s\right] \\
\stackrel{6.3 .6 \mathrm{~b}}{=} & {\left[m \otimes n \cdot s, m \otimes n^{\prime} \cdot s, m \otimes n^{\prime \prime} \cdot s\right] } \\
& =\left[\rho_{s}(m, n), \rho_{s}\left(m, n^{\prime}\right), \rho_{s}\left(m, n^{\prime \prime}\right)\right], \\
\rho_{s}((m \cdot t, n)) & =m \cdot t \otimes n \cdot s \stackrel{6.3 .6 \mathrm{c}}{-} m \otimes t \cdot(n \cdot s)=m \otimes(t \cdot n) \cdot s=\rho_{s}(m, t \cdot n),
\end{aligned}
$$

for all $m, m^{\prime}, m^{\prime \prime} \in M, n, n^{\prime}, n^{\prime \prime} \in N, t \in T$. That is to say, $\rho_{s}$ is a $T$-balanced bilinear map and hence it factors uniquely through $M \otimes_{T} N$ via the heap morphism

$$
\varrho_{s}: M \otimes_{T} N \longrightarrow M \otimes_{T} N, \quad m \otimes n \longmapsto m \otimes n \cdot s .
$$

Now, consider the assignment

$$
\varrho: S \longrightarrow E\left(M \otimes_{T} N\right), \quad s \longmapsto \varrho_{s} .
$$

For all $m \in M, n \in N, s, s^{\prime}, s^{\prime \prime} \in S$,

$$
\begin{aligned}
\varrho_{\left[s, s^{\prime}, s^{\prime \prime}\right]}(m \otimes n) & =m \otimes n \cdot\left[s, s^{\prime}, s^{\prime \prime}\right] \stackrel{\left[\frac{4.1 .1 \mathrm{bb}}{-}\right.}{=} m \otimes\left[n \cdot s, n \cdot s^{\prime}, n \cdot s^{\prime \prime}\right] \\
& \stackrel{[6.3 .6 \mathrm{~b}}{-}\left[m \otimes n \cdot s, m \otimes n \cdot s^{\prime}, m \otimes n \cdot s^{\prime \prime}\right] \\
& =\left[\varrho_{s}(m \otimes n), \varrho_{s^{\prime}}(m \otimes n), \varrho_{s^{\prime \prime}}(m \otimes n)\right] \\
& =\left[\varrho_{s}, \varrho_{s^{\prime}}, \varrho_{s^{\prime \prime}}\right](m \otimes n), \\
\varrho_{s s^{\prime}}(m \otimes n) & =m \otimes n \cdot s s^{\prime}=m \otimes(n \cdot s) \cdot s^{\prime}=\varrho_{s^{\prime}}(m \otimes(n \cdot s)) \\
& =\left(\varrho_{s^{\prime}} \circ \varrho_{s}\right)(m \otimes n) .
\end{aligned}
$$

Therefore, $\varrho: S^{o p} \longrightarrow E\left(M \otimes_{T} N\right)$ is a morphism of trusses and hence $M \otimes_{T} N$ is a right $S$-module. If $S$ admits an identity 1 and $N$ is a unital $S$-module, then $\varrho(1)=1_{M \otimes N}$ and hence $\varrho$ (and $M \otimes_{T} N$ ) is also unital.

The other case is proven in a symmetric way.

2. Similarly to the proof of statement 1, one considers the assignment

$$
\varphi^{\prime}: M \times N \longrightarrow M^{\prime} \otimes_{T} N, \quad(m, n) \longmapsto \varphi(m) \otimes n .
$$


Since $\varphi$ is a morphism of right $T$-modules, $\varphi^{\prime}$ is a $T$-balanced bilinear map, and hence it factors uniquely through

$$
\varphi \otimes N: M \otimes_{T} N \longrightarrow M^{\prime} \otimes_{T} N, \quad m \otimes n \longmapsto \varphi(m) \otimes n .
$$

Since $\varphi \otimes N$ acts trivially on the elements in $N$, and the $S$-actions on $M \otimes_{T} N$ and $M^{\prime} \otimes_{T} N$ are defined using the $S$-action on $N$ only, the resulting map is a homomorphism of right $S$-modules. The other case is proven in a symmetric way.

3. This follows immediately from assertions 1 and 2 .

Proposition 6.3.9. Let $T, S$ be trusses and let $M$ be a T-S-bimodule. Then the tensor functor $-\otimes_{T} M: \bmod -T \longrightarrow \bmod -S$ is the left adjoint to the functor $\operatorname{Hom}_{T}(M,-)$.

Proof. The proof of this proposition follows the same arguments as the proof of the corresponding statement for modules over rings. The only difference is that the distributivity of the tensor product over the heap ternary operation (rather than over a binary addition) should be employed whenever necessary (for example in showing that the unit and counit of the adjunction are morphisms of heaps). The unit and the counit of the adjunction are explicitly given by

$$
\begin{gathered}
\eta_{X}: X_{T} \longrightarrow \operatorname{Hom}_{S}\left(M_{S}, X \otimes_{T} M_{S}\right), \quad x \longmapsto[m \longmapsto x \otimes m], \\
\varepsilon_{Y}: \operatorname{Hom}_{S}\left(M_{S}, Y_{S}\right) \otimes_{T} M_{S} \longmapsto Y_{S}, \quad\left[f_{i} \otimes m_{i}\right]_{i=1}^{2 n+1} \longmapsto\left[f_{i}\left(m_{i}\right)\right]_{i=1}^{2 n+1},
\end{gathered}
$$

for all right $T$-modules $X$ and right $S$-modules $Y$. Now, one can easily show that the functors, unit and counit fulfill assumptions of Theorem A.0.18. Therefore we have that $-\otimes_{T} M \dashv \operatorname{Hom}_{T}(M,-)$.

Corollary 6.3.10. Let $R, S, T, U$ be trusses and let $A$ be an $R$-S-bimodule, $B$ be an $S$-T-bimodule and $C$ be a $T$-U-bimodule. Then the map,

$$
\begin{gathered}
\alpha_{A, B, C}:\left(A \otimes_{R} B\right) \otimes_{T} C \longrightarrow A \otimes_{R}\left(B \otimes_{T} C\right), \\
(a \otimes b) \otimes c \longmapsto a \otimes(b \otimes c) .
\end{gathered}
$$

is an isomorphism of $R$-U-bimodules. 
Proof. The assertion follows from Proposition 6.3.9 by standard arguments.

In view of the associativity of tensor products stemming from Corollary 6.3 .10 we no longer need to write brackets in-between multiple tensor products.

The distributive laws for a truss $T$ mean that the multiplication map $\mu: T \times T \longrightarrow T$, $(s, t) \longmapsto s t$ is bilinear. Hence, there is a unique heap homomorphism $\hat{\mu}: T \otimes T \longrightarrow T$. The associative law for $\mu$ is then reflected by the commutativity of the following diagram:

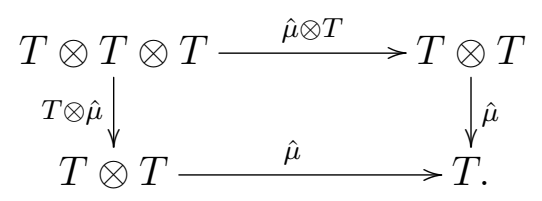

The existence of a map $\hat{\mu}$ satisfying (6.3.7) can be taken as the definition of the truss, provided that one carefully explains the meaning of $\otimes$ (for example, by resorting to relations (6.3.6a and (6.3.6b) without referring to trusses, in order to avoid the ignotum per ignotius trap.

Similarly, if $T$ is a truss and $M$ a left $T$-module with action $\lambda_{M}$, then conditions (4.1.1b) and 4.1.1c) mean that $\lambda_{M}: T \times M \longrightarrow M$ is a bilinear map, so it induces a unique map $\hat{\lambda}_{M}: T \otimes M \longrightarrow M$. Thus, exactly as in the case of modules over rings, a left module over a truss $T$ can be equivalently defined as an Abelian heap $M$ together with a heap homomorphism $\hat{\lambda}_{M}: T \otimes M \longrightarrow M$ such that

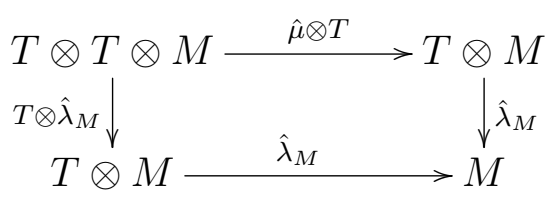

commutes, where $\hat{\mu}$ is the multiplication in $T$. In a similar way, a right $T$-module can be equivalently described as a heap $M$ together with an associative right action $\hat{\varrho}_{M}: M \otimes T \longrightarrow M$. Taking these equivalent definitions of modules into account, one can interpret the tensor product as a coequalizer.

Proposition 6.3.11. Let $T$ be a truss. For a right $T$-module $M$ and left $T$-module $N$, the tensor product $M \otimes_{T} N$ is the coequalizer of the following diagram of Abelian heaps

$$
M \otimes T \otimes N \underset{\hat{\varrho}_{M} \otimes N}{\stackrel{\hat{\lambda}_{N}}{\longrightarrow}} M \otimes N,
$$


where $\hat{\varrho}_{M}$ and $\hat{\lambda}_{N}$ are the corresponding actions.

Proof. Consider the structural morphisms $\phi: M \times N \longrightarrow M \otimes N$ and $\varphi: M \times N \longrightarrow$ $M \otimes_{T} N$, part of the tensor product data. By definition, $\varphi$ is a bilinear map and so it factors uniquely through the morphism of Abelian heaps

$$
\tilde{\varphi}: M \otimes N \longrightarrow M \otimes_{T} N,
$$

such that $\tilde{\varphi} \circ \phi=\varphi$. In addition, $\tilde{\varphi}$ satisfies

$$
\begin{aligned}
\tilde{\varphi}\left(\left(\hat{\varrho}_{M} \otimes N\right)(m \otimes t \otimes n)\right) & =\tilde{\varphi}(m \cdot t \otimes n)=\tilde{\varphi}(\phi(m \cdot t, n))=\varphi(m \cdot t, n) \\
& =\varphi(m, t \cdot n)=\tilde{\varphi}\left(\left(M \otimes \hat{\lambda}_{N}\right)(m \otimes t \otimes n)\right),
\end{aligned}
$$

for all $m \in M, n \in N, t \in T$, because $\varphi$ is $T$-balanced. Since every morphism involved is a morphism of Abelian heaps, we conclude that $\tilde{\varphi}$ coequalizes the pair (6.3.8). Now, let $(Q, q: M \otimes N \rightarrow Q)$ be a pair coequalizing 6.3.8) as well. The composition $q \circ \phi$ is bilinear because

$$
\begin{aligned}
(q \circ \phi)\left(\left[m, m^{\prime}, m^{\prime \prime}\right], n\right) & =q\left(\left[m, m^{\prime}, m^{\prime \prime}\right] \otimes n\right) \stackrel{\sqrt[6.3 .6 a)]{-}}{-} q\left(\left[m \otimes n, m^{\prime} \otimes n, m^{\prime \prime} \otimes n\right]\right) \\
& =\left[q(m \otimes n), q\left(m^{\prime} \otimes n\right), q\left(m^{\prime \prime} \otimes n\right)\right] \\
& =\left[(q \circ \phi)(m, n),(q \circ \phi)\left(m^{\prime}, n\right),(q \circ \phi)\left(m^{\prime \prime}, n\right)\right],
\end{aligned}
$$

for all $m, m^{\prime}, m^{\prime \prime} \in M, n \in N$, and analogously on the other side. Furthermore, it is also $T$-balanced because $q$ coequalizes (6.3.8), and hence

$$
\begin{aligned}
(q \circ \phi)(m \cdot t, n) & =q(m \cdot t \otimes n)=q\left(\left(\hat{\varrho}_{M} \otimes N\right)(m \otimes t \otimes n)\right) \\
& =q\left(\left(M \otimes \hat{\lambda}_{N}\right)(m \otimes t \otimes n)\right)=(q \circ \phi)(m, t \cdot n),
\end{aligned}
$$

for all $m \in M, n \in N, t \in T$. Thus, there exists a unique morphism of Abelian heaps $\tilde{q}: M \otimes_{T} N \rightarrow Q$ such that $\tilde{q} \circ \varphi=q \circ \phi$. In particular, $\tilde{q} \circ \tilde{\varphi} \circ \phi=q \circ \phi$ (by definition of $\tilde{\varphi}$ ) and since both $q$ and $\tilde{q} \circ \tilde{\varphi}$ are heap homomorphisms, the uniqueness part of the universal property of the tensor product entails that $\tilde{q} \circ \tilde{\varphi}=q$. Summing up, the pair $\left(M \otimes_{T} N, \tilde{\varphi}\right)$ is the coequalizer of (6.3.8) in $\mathbf{A h}$.

Remark 6.3.12. We know from [17, Theorem 9.4.14] (see Theorem C.0.16) that the category of modules over a truss is complete and cocomplete, see Definition A.0.33. 
Let $T$ be a truss and $T_{u}$ be its unital extension as in Section 5.1.2. By [24, Chapter VII, Section 4], the functor $T_{u} \otimes-: \mathbf{A} \mathbf{h} \longrightarrow\left(T_{u}\right)_{1}$ - $\mathbf{m o d}$ is left adjoint to the forgetful functor $\mathrm{U}^{\prime}:\left(T_{u}\right)_{1}$ - $\mathbf{m o d} \longrightarrow \mathbf{A h}$ (and hence it is called the free unital $T_{u}$-module functor). Since the functor $\mathrm{E}_{1}: T-\bmod \longrightarrow\left(T_{u}\right)_{1}$ - $\bmod$ of Theorem 6.1 .2 is the inverse of the restriction of scalars $\mathrm{S}_{T}:\left(T_{u}\right)_{1}$-mod $\longrightarrow T$-mod and since clearly $\mathrm{U}^{\prime} \circ \mathrm{E}_{1}$ coincides with the forgetful functor $\mathrm{U}: T$ - $\mathbf{m o d} \longrightarrow \mathbf{A h}$, the composition $\mathrm{S}_{T} \circ\left(T_{u} \otimes-\right)$ is left adjoint to $\mathrm{U}$. Once observed that $\mathrm{S}_{T} \circ\left(T_{u} \otimes-\right)$ is naturally isomorphic to tensoring by the left $T$-module $\mathrm{S}_{T}\left(T_{u}\right)$, we conclude that the functor $\mathrm{S}_{T}\left(T_{u}\right) \otimes-: \mathbf{A h} \longrightarrow T$ - $\mathbf{m o d}$ is left adjoint to the forgetful functor $\mathrm{U}$ and hence it is called the free $T$-module functor.

Proposition 6.3.13. Let $X$ be a non-empty set and $T$ be a unital truss. Denote by $\mathcal{A}(X)$ the free Abelian heap over $X$ and by $\mathcal{T}^{X}$ the free unital T-module over $X$. Then $\mathcal{T}^{X} \cong T \otimes \mathcal{A}(X)$ as $T$-modules. In particular, the following diagram of functors commutes

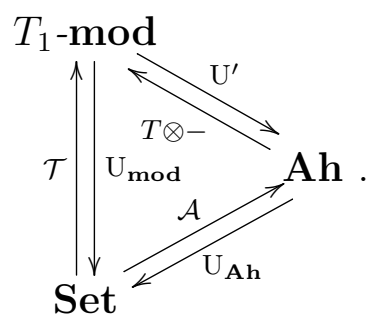

Proof. Fix $e \in X$. In view of Corollary 1.4 .6 we know that

$$
\mathcal{A}(X) \cong \mathrm{H}\left(\bigoplus_{x \in X \backslash\{e\}} \mathbb{Z}\right) \cong{\underset{x \in X}{\boxplus}}_{\mathcal{A}} \mathcal{A}(\{x\}),
$$

and the isomorphism $\mathcal{A}(X) \cong \underset{x \in X}{\boxplus} \mathcal{A}(\{x\})$ is independent from the choice of $e \in X$. Now, since $T \otimes-$ is cocontinuous (because it is the left adjoint of the forgetful functor), we have the following chain of isomorphisms of left $T$-modules

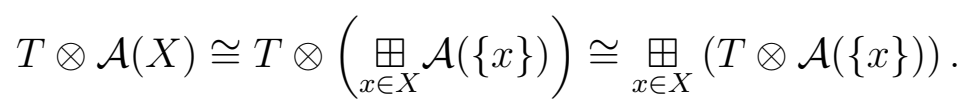

Consider $\mathcal{A}(\{x\})$. As a set, $\mathcal{A}(\{x\})=\{x\}$ with the ternary operation $[x, x, x]=x$. This makes it clear that $\{1\} \longrightarrow \mathcal{A}(\{x\}), 1 \longmapsto x$, is an isomorphism of (Abelian) heaps. Therefore, $T \otimes \mathcal{A}(X) \cong \underset{x \in X}{\boxplus} T x \cong \mathcal{T}^{X}$. 
Let us make explicit the foregoing isomorphism in an extremely easy example.

Example 6.3.14. Let $X=\{a, b\}$ be a set with two elements. The free Abelian heap $\mathcal{A}(X)$ on $X$ can be realized as the set

$$
\{a, b, a b a, b a b, a b a b a, b a b a b, a b a b a b a, b a b a b a b, \ldots\}
$$

with bracket given by concatenation and (symmetric) pruning. Then, for instance,

$$
t \otimes a b a b a \longleftrightarrow(t a)(t b)(t a)(t b)(t a)=([t, 1, t, 1, t] a)([t, 1, t] b)(1 a)(1 b)(1 a)
$$

Corollary 6.3.15 (of Proposition 6.3.13). Let $T$ be a truss and $T_{u}$ its unital extension. Denote by $\mathrm{U}_{\text {mod }}: T$-mod $\longrightarrow$ Set the forgetful functor. In the following diagram of adjunctions, the subdiagram involving only the right adjoints is commutative

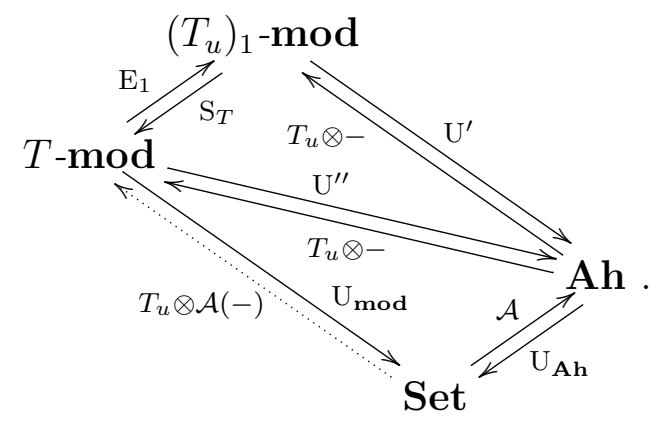

In particular, the free $T$-module over a set $X$ is $T_{u} \otimes \mathcal{A}(X)$.

Concretely, when $T$ is a not necessarily unital truss we can describe the free $T$-module over a set $X$ as the direct sum of Abelian heaps

$$
\mathcal{T}^{X}:=\underset{x \in X}{\boxplus} T_{u} x
$$

with the $T$-action given component-wise, that is,

$$
t \cdot\left[z_{1} x_{1}, \ldots, z_{2 k+1} x_{2 k+1}\right]=\left[\left(\iota_{T}(t) z_{1}\right) x_{1}, \ldots,\left(\iota_{T}(t) z_{2 k+1}\right) x_{2 k+1}\right]
$$

for all $x_{1}, \ldots, x_{2 k+1} \in X, z_{1}, \ldots, z_{2 k+1} \in T_{u}$ and $t \in T$. The canonical map $\iota_{X}: X \longrightarrow$ $\mathcal{T}^{X}$ (that is, the unit of the adjunction $T_{u} \otimes \mathcal{A}(-) \dashv \mathrm{U}_{\text {mod }}$ ) sends every $x \in X$ to 
$1 x \in T_{u} x$. The other way around, the counit $\epsilon$ of the adjunction $T_{u} \otimes \mathcal{A}(-) \dashv \mathrm{U}_{\text {mod }}$ realizes every $T$-module $M$ as a quotient of a free one:

$$
\mathcal{T}^{\mathrm{U}_{\text {mod }}(M)} \cong T_{u} \otimes \mathcal{A}\left(\mathrm{U}_{\text {mod }}(M)\right) \stackrel{\epsilon_{M}}{\longrightarrow} M
$$

(since $\mathcal{F}$ is faithful, every component of $\epsilon$ is full in view of [24, Theorem IV.3.1])(see A.0.35 


\section{Chapter 7}

\section{Morita theory and projective modules}

In the final chapter, we introduce the Morita theory for trusses. In rings we say that two rings are Morita equivalent if their categories of left modules are equivalent in the categorical sense. We present an analogue of that theory for trusses. It is worth mentioning that the lack of an absorber in modules complicates the theory significantly. The important tools to study Morita theory for rings are provided by projective modules. Therefore, further, we study projective modules over trusses.

In Section 7.1, we introduce the Morita theory for trusses. The main result of this section is the Eilenberg-Watts Theorem for trusses. The Eilenberg-Watts Theorem tells us that an equivalence between categories of modules over trusses is given by a bimodule tensoring. Further, we discover that this bimodule must satisfy property which we name the dual basis property. Modules that satisfy dual basis property are called tiny.

In Section 7.2, we study projective modules over trusses. Projective modules are of great importance for the Morita theory since every tiny module is projective. To study projective modules we introduce exact sequences. Since category of modules over a truss is not Abelian, it is not obvious how to define an exact sequence in general. Further, we study when an exact sequence splits. We use splittings of sequences to give conditions for a module to be projective or tiny in terms of decomposition into a 
product and coproduct od modules.

Both sections are part of [33].

\subsection{Morita theory and modules over trusses}

Given two trusses $S, T$ and a $T$-S-bimodule $M$ we already know that the functor $M \otimes_{S}-: S-\bmod \longrightarrow T$-mod is left adjoint to the functor $\operatorname{Hom}_{T}(M,-): T$-mod $\longrightarrow$ $S$-mod. Our aim in the present section is to show that, if $T$ and $S$ are (unital) trusses, then any heap functor $\mathrm{L}: S$-mod $\longrightarrow T$-mod which admits a right adjoint is of the form $P \otimes_{S}-$ for a suitable (unital) $T$-S-bimodule $P$. Recall that a functor F $: S$-mod $\longrightarrow T$-mod is a heap functor provided that, for all $M, N \in S$-mod, the functions $\mathrm{F}_{M, N}$ defined by equation 4.1.3 are morphisms of heaps. Recall also that the unital extension $T_{u}$ of a truss $T$ is a $T$ - $T$-bimodule via the truss homomorphism $\iota_{T}: T \longrightarrow T_{u}$.

Lemma 7.1.1. Let $S, T$ be trusses and let $\mathrm{F}: S-\bmod \longrightarrow T-\bmod$ be a heap functor between their categories of modules. Then $P:=\mathrm{F}\left(\mathrm{S}_{S}\left(S_{u}\right)\right)$ is a T-S-bimodule. Furthermore, if $S$ is unital and $\mathrm{F}: S_{1}-\bmod \longrightarrow T-\bmod$ is a heap functor, then $P^{\prime}:=\mathrm{F}(S)$ is a T-S-bimodule which is unital as right $S$-module.

Proof. To simplify notation we write $S_{u}$ instead of $\mathrm{S}_{S}\left(S_{u}\right)$. For every $s \in S$, consider the left $S$-module morphism

$$
\rho_{s}: S_{u} \longrightarrow S_{u}, \quad z \longmapsto z \cdot \iota_{S}(s)
$$

Clearly, $\rho_{s s^{\prime}}=\rho_{s^{\prime}} \circ \rho_{s}$ and, by the right distributive law of the action of $S$ on $S_{u}$, $\rho_{\left[s, s^{\prime}, s^{\prime \prime}\right]}=\left[\rho_{s}, \rho_{s^{\prime}}, \rho_{s^{\prime \prime}}\right]$ in $E_{S}\left(S_{u}\right):=\operatorname{Hom}_{S}\left(S_{u}, S_{u}\right)$ for all $s, s^{\prime}, s^{\prime \prime} \in S$. Therefore the map

$$
\rho: S^{o p} \longrightarrow E_{S}\left(S_{u}\right), \quad s \longmapsto \rho_{s},
$$

is a homomorphism of trusses, where $S^{o p}$ is the opposite truss from Definition 6.3.5. Since $\mathrm{F}$ is a heap functor, the composite

$$
S^{o p} \stackrel{\rho}{\longrightarrow} E_{S}\left(S_{u}\right) \stackrel{F_{S_{u}, S_{u}}}{\longrightarrow} E_{T}\left(\mathrm{~F}\left(S_{u}\right)\right)=E_{T}(P),
$$


where $\mathrm{F}_{S_{u}, S_{u}}$ is defined by (4.1.3), is a morphism of trusses. As a consequence, $P$ inherits the structure of a $T$-S-bimodule. If $S$ is unital, we may perform the same construction using $S$ instead of $S_{u}$ and $P^{\prime}=\mathrm{F}(S)$ becomes unital as a right $S$-module.

Proposition 7.1.2. Let $S, T$ be trusses. A heap functor $\mathrm{L}: S$-mod $\longrightarrow T$-mod admits a right adjoint if and only if it is naturally equivalent to $P \otimes_{S}-$ for a suitable T-S-bimodule P. Namely, $P:=\mathrm{L}\left(\mathrm{S}_{S}\left(S_{u}\right)\right)$. If, in addition, $S$ is unital then a heap functor $\mathrm{L}: S_{1}-\bmod \longrightarrow T-\bmod$ admits a right adjoint if and only if it is naturally equivalent to $P^{\prime} \otimes_{S}-$ for a suitable $T$-S-bimodule $P^{\prime}$, unital as a right $S$-module. Namely, $P^{\prime}:=\mathrm{L}(S)$.

Proof. We already know from Lemma 7.1.1 that $P:=\mathrm{L}\left(\mathrm{S}_{S}\left(S_{u}\right)\right)$ is a $T$-S-bimodule. Let us denote by $\mathrm{R}: T$-mod $\longrightarrow S$-mod the right adjoint to $L$ and let us consider the adjunction isomorphism

$$
\Phi_{S_{u}, N}: \operatorname{Hom}_{T}(P, N)=\operatorname{Hom}_{T}\left(\mathrm{~L}\left(\mathrm{~S}_{S}\left(S_{u}\right)\right), N\right) \cong \operatorname{Hom}_{S}\left(\mathrm{~S}_{S}\left(S_{u}\right), \mathrm{R}(N)\right)
$$

for all $N$ in $T$-mod. Then, for all $s \in S$ and $f \in \operatorname{Hom}_{T}(P, N)$,

$$
\begin{aligned}
\Phi_{S_{u}, N}(s \cdot f) & =\Phi_{S_{u}, N}\left(f \circ \mathrm{L}\left(\rho_{s}\right)\right)=\left(\Phi_{S_{u}, N} \circ \operatorname{Hom}_{T}\left(\mathrm{~L}\left(\rho_{s}\right), N\right)\right)(f) \\
& =\left(\operatorname{Hom}_{S}\left(\rho_{s}, \mathrm{R}(N)\right) \circ \Phi_{S_{u}, N}\right)(f) \\
& =\Phi_{S_{u}, N}(f) \circ \rho_{s}=s \cdot \Phi_{S_{u}, N}(f),
\end{aligned}
$$

that is $\Phi_{S_{u}, N}$ is a left $S$-linear isomorphism natural in $N \in T$-mod. Since $\mathrm{S}_{S}$ is the inverse of $\mathrm{E}_{1}$, we have further

$$
\operatorname{Hom}_{S}\left(\mathrm{~S}_{S}\left(S_{u}\right), \mathrm{R}(N)\right) \cong \mathrm{S}_{S}\left(\operatorname{Hom}_{S_{u}}\left(S_{u}, \mathrm{E}_{1}(\mathrm{R}(N))\right)\right)
$$

as left $S$-modules. Now, in view of the fact that both $S_{u}$ and $\mathrm{E}_{1}(\mathrm{R}(N))$ are unital, the assignment

$$
\operatorname{Hom}_{S_{u}}\left(S_{u}, \mathrm{E}_{1}(\mathrm{R}(N))\right) \longrightarrow \mathrm{E}_{1}(\mathrm{R}(N)), \quad f \longmapsto f\left(1_{S_{u}}\right)
$$

is an isomorphism of heaps, natural in $N$, which is also left $S_{u}$-linear. Therefore,

$$
\mathrm{S}_{S}\left(\operatorname{Hom}_{S_{u}}\left(S_{u}, \mathrm{E}_{1}(\mathrm{R}(N))\right)\right) \cong \mathrm{S}_{S}\left(\mathrm{E}_{1}(\mathrm{R}(N))\right) \cong \mathrm{R}(N)
$$


and we conclude that $\mathrm{R} \cong \operatorname{Hom}_{T}(P,-)$ as functors from $T$-mod to $S$-mod. Being the left adjoint to $\operatorname{Hom}_{T}(P,-), \mathrm{L} \cong P \otimes_{S}-$ as desired, by the uniqueness of adjoints up to isomorphism. Finally, in case $S$ is unital one may mimic the same procedure starting with $P^{\prime}=\mathrm{L}(S)$ instead.

With Proposition 7.1.2 we have shown that any functor between module categories over trusses which admits a right adjoint is naturally obtained by taking tensor products with suitable bimodules. Now we prove an analogue of the Eilenberg-Watts theorem for modules over trusses which, in turn, allows us to give an intrinsic characterisation of when a functor is given by tensoring by a bimodule (and hence it is a left adjoint) in terms of properties of the functor itself.

Theorem 7.1.3 (Eilenberg-Watts Theorem for trusses). Let $T$ and $S$ be trusses. If $\mathrm{F}: T-\bmod \longrightarrow S-\bmod$ is a cocontinuous heap functor, then

$$
\mathrm{F}(-) \cong P \otimes_{T}-,
$$

for an $S$-T-bimodule $P$. Namely, $P:=\mathrm{F}\left(\mathrm{S}_{T}\left(T_{u}\right)\right)$. If, in addition, $T$ is unital and $\mathrm{F}: T_{1}-\bmod \longrightarrow S$-mod is a cocontinuous heap functor, then

$$
\mathrm{F}(-) \cong P^{\prime} \otimes_{T}-
$$

for an $S$-T-bimodule $P^{\prime}$, unital as right $T$-module. Namely, $P^{\prime}:=\mathrm{F}(T)$.

Proof. We prove only the first claim and, for the sake of simplicity, we write $T_{u}$ instead of $\mathrm{S}_{T}\left(T_{u}\right)$. Let $X$ be a $T$-module. One can consider a coequalizer diagram

$$
\operatorname{Ker}(\pi) \stackrel{p_{1}}{\underset{p_{2}}{\longrightarrow}} \mathcal{T}^{X} \stackrel{\pi}{\longrightarrow} X,
$$

as in the proof of Proposition 4.4.2, where $\mathcal{T}^{X}$ is the free $T$-module over the set underlying $X, \pi$ is the canonical epimorphism, $\operatorname{Ker}(\pi)=\left\{(x, y) \in \mathcal{T}^{X} \times \mathcal{T}^{X} \mid \pi(x)=\pi(y)\right\}$ with the component-wise $T$-module structure, and $p_{1}, p_{2}$ are the (restrictions of the) two canonical projections. One can extend diagram (7.1.1) to 


$$
\mathcal{T}^{\operatorname{Ker}(\pi)} \underset{p_{2}^{\prime}}{\stackrel{p_{1}^{\prime}}{\longrightarrow}} \mathcal{T}^{X} \stackrel{\pi}{\longrightarrow} X,
$$

where $\pi^{\prime}: \mathcal{T}^{\operatorname{Ker}(\pi)} \longrightarrow \operatorname{Ker}(\pi), p_{1}^{\prime}=p_{1} \circ \pi^{\prime}$ and $p_{2}^{\prime}=p_{2} \circ \pi^{\prime}$. Since $\pi^{\prime}$ is an epimorphism, (7.1.2) is a coequalizer diagram as well. By Lemma 7.1.1, $P:=\mathrm{F}\left(T_{u}\right)$ inherits the structure of an $S$-T-bimodule from the fact that $\mathrm{F}$ is a heap functor. Since F is a cocontinuous functor and in view of Proposition 6.3.7, there is the following chain of natural isomorphisms:

$$
\mathrm{F}\left(\mathcal{T}^{X}\right)=\mathrm{F}\left(\underset{x \in X}{\boxplus} T_{u}\right) \cong \underset{x \in X}{\boxplus} \mathrm{F}\left(T_{u}\right) \cong{\underset{x \in X}{\boxplus}}\left(P \otimes_{T} T_{u}\right) \cong P \otimes_{T} \underset{x \in X}{\boxplus} T_{u}=P \otimes_{T} \mathcal{T}^{X} .
$$

Moreover we can fill in a diagram

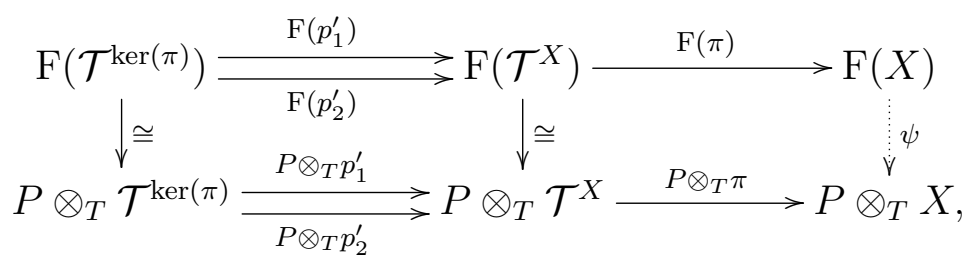

where both horizontal diagrams are coequalizers obtained from $(7.1 .2)$, because $\mathrm{F}$ and $P \otimes_{T}$ - preserve colimits, and $\psi$ is the isomorphism induced by their universal property. It can be checked, by resorting to the uniqueness of the morphisms induced at the level of the coequalizers, that $\psi$ is in fact natural in $X$.

Corollary 7.1.4. Let $T, S$ be trusses. A functor $\mathrm{F}: T$-mod $\longrightarrow S$-mod is a left adjoint if and only if it is a cocontinuous heap functor. If, in addition, $T$ is unital then $\mathrm{F}: T_{1}$-mod $\longrightarrow S$-mod is a left adjoint if and only if it is a cocontinuous heap functor.

Proof. The statements follow from Proposition 7.1.2. Theorem 7.1.3 and the fact that $P \otimes_{T}-$ is cocontinuous, heap and a left adjoint functor.

Assume that $S$ and $T$ are unital trusses. A key question related to the Morita theory for trusses is what can be said when $T_{1}$-mod $\cong S_{1}$-mod. Notice that this covers the non-unital case as well, since in that case $T$-mod $\cong S$-mod if and only if $\left(T_{u}\right)_{1}-\bmod \cong\left(S_{u}\right)_{1}-\mathbf{m o d}$. 
Theorem 7.1.5. Let $T, S$ be unital trusses. The following statement are equivalent:

1. $T_{1}$-mod $\cong S_{1}$-mod, where equivalence is a heap functor.

2. There exist unital bimodules ${ }_{S} P_{T}$ and ${ }_{T} Q_{S}$ together with an S-bilinear isomorphism ev $: P \otimes_{T} Q \longrightarrow S$ and a $T$-bilinear isomorphism $\mathrm{db}: T \longrightarrow Q \otimes_{S} P$ such that

$$
\left(Q \otimes_{S} \mathrm{ev}\right) \circ\left(\mathrm{db} \otimes_{T} Q\right)=1_{Q} \quad \text { and } \quad\left(\mathrm{ev} \otimes_{S} P\right) \circ\left(P \otimes_{T} \mathrm{db}\right)=1_{P}
$$

3. There exist unital bimodules ${ }_{S} P_{T}$ and ${ }_{T} Q_{S}$ together with an $S$-bilinear isomorphism $\mathrm{db}^{\prime}: S \longrightarrow P \otimes_{T} Q$ and a T-bilinear isomorphism $\mathrm{ev}^{\prime}: Q \otimes_{S} P \longrightarrow T$ such that

$$
\left(P \otimes_{T} \mathrm{ev}^{\prime}\right) \circ\left(\mathrm{db}^{\prime} \otimes_{S} P\right)=1_{P} \quad \text { and } \quad\left(\mathrm{ev}^{\prime} \otimes_{T} Q\right) \circ\left(Q \otimes_{S} \mathrm{db}^{\prime}\right)=1_{Q} .
$$

Proof. Since the proofs of $1 \Longleftrightarrow 2$ and of $11 \Longleftrightarrow 3$ are similar, we will present explicitly only the first one and leave the second one to the reader.

To show that 1 implies 2 assume that $\mathrm{L}: T_{1}-\bmod \longrightarrow S_{1}-\bmod$ and $\mathrm{R}: S_{1}-\bmod \longrightarrow$ $T_{1}$-mod are inverse equivalences (or quasi-inverse functors). Equivalently, we may assume that $\mathrm{L}$ is left adjoint to $\mathrm{R}$ and that the counit $\varepsilon: \mathrm{L} \circ \mathrm{R} \longrightarrow$ id and the unit $\eta: \mathrm{id} \longrightarrow \mathrm{R} \circ \mathrm{L}$ of this adjunction are natural isomorphisms. In light of Proposition 7.1.2. there exists a unital $S$-T-bimodule $P$ such that $\mathrm{L} \cong P \otimes_{T}-$. At the same time, we may look at $\mathrm{R}$ as left adjoint to $\mathrm{L}$ with counit $\eta^{-1}: \mathrm{R} \circ \mathrm{L} \longrightarrow$ id and unit $\varepsilon^{-1}:$ id $\longrightarrow \mathrm{L} \circ \mathrm{R}$, and hence there exists a unital $T$-S-bimodule $Q$ such that $\mathrm{R} \cong Q \otimes_{S}-$. Consider the following isomorphisms

$$
\begin{aligned}
& \mathrm{db}:=\left(T \stackrel{\eta_{T}}{\longrightarrow} \mathrm{R}\left(P \otimes_{T} T\right) \stackrel{\cong}{\longrightarrow} Q \otimes_{S} P \otimes_{T} T \stackrel{\cong}{\longrightarrow} Q \otimes_{S} P\right), \\
& \mathrm{ev}:=\left(P \otimes_{T} Q \stackrel{\cong}{\longrightarrow} P \otimes_{T} Q \otimes_{S} S \stackrel{\cong}{\longrightarrow} P \otimes_{T} \mathrm{R}(S) \stackrel{\varepsilon_{S}}{\longrightarrow} S\right) .
\end{aligned}
$$

First, we are going to show that $\eta$ and $\varepsilon$ can be written in terms of ev and $\mathrm{db}$. Then, we will see how the triangular identities for unit and counit reflect on ev and $\mathrm{db}$. For every left $T$-module $M$ and for every $m \in M$, consider the left $T$-module homomorphism $\rho_{m}: T \longrightarrow M, t \longmapsto t \cdot m$. By naturality of $\eta$,

$$
\eta_{M}(m)=\left(\eta_{M} \circ \rho_{m}\right)\left(1_{T}\right)=\left(Q \otimes_{S} P \otimes_{T} \rho_{m}\right)\left(\eta_{T}\left(1_{T}\right)\right)=\mathrm{db}\left(1_{T}\right) \otimes_{T} m
$$


Similarly, for every left $S$-module $N$ and for every $n \in N$ we consider the left $S$-module homomorphism $\rho_{n}: S \longrightarrow N, s \longmapsto s \cdot n$ and, by naturality of $\varepsilon$,

$$
\begin{gathered}
\varepsilon_{N}\left(p \otimes_{T} q \otimes_{S} n\right)=\left(\varepsilon_{N} \circ\left(P \otimes_{T} Q \otimes_{S} \rho_{n}\right)\right)\left(p \otimes_{T} q \otimes_{S} 1_{S}\right) \\
=\rho_{n}\left(\varepsilon_{S}\left(p \otimes_{T} q \otimes_{S} 1_{S}\right)\right)=\operatorname{ev}\left(p \otimes_{T} q\right) \cdot n .
\end{gathered}
$$

Let us write explicitly $\mathrm{db}\left(1_{T}\right)=\left[q_{i} \otimes_{S} p_{i}\right]_{i}$ and $\operatorname{ev}\left(p \otimes_{T} q\right)=q(p)$. By the triangular identities (see diagrams in Theorem A.0.18), for every $S$-module $N$ and for all $q \in$ $Q, n \in N$,

$$
\begin{aligned}
q \otimes_{S} n & =\left(\left(Q \otimes_{S} \varepsilon_{N}\right) \circ \eta_{Q \otimes_{S} N}\right)\left(q \otimes_{S} n\right)=\left(Q \otimes_{S} \varepsilon_{N}\right)\left(\left[q_{i} \otimes_{S} p_{i}\right]_{i} \otimes_{T} q \otimes_{S} n\right) \\
& =\left[q_{i} \cdot q\left(p_{i}\right)\right]_{i} \otimes_{S} n .
\end{aligned}
$$

In a similar way, for every $T$-module $M$ and for all $p \in P$ and $m \in M$,

$$
\begin{aligned}
p \otimes_{T} m & =\left(\varepsilon_{P \otimes_{T} M} \circ\left(P \otimes_{T} \eta_{M}\right)\right)\left(p \otimes_{T} m\right)=\varepsilon_{P \otimes_{T} M}\left(p \otimes_{T}\left[q_{i} \otimes_{S} p_{i}\right]_{i} \otimes_{S} m\right) \\
& =\left[q_{i}(p) \cdot p_{i}\right]_{i} \otimes_{T} m .
\end{aligned}
$$

In particular, for $N=S, n=1_{S}, M=T, m=1_{T}$, we find that

$$
\left[q_{i} \cdot q\left(p_{i}\right)\right]_{i}=q \quad \text { and } \quad\left[q_{i}(p) \cdot p_{i}\right]_{i}=p
$$

for all $p \in P$ and $q \in Q$. Concerning bilinearity, on the one hand, for every $t \in T$,

$$
\begin{gathered}
{\left[q_{i} \otimes_{S} p_{i}\right]_{i} \cdot t=\left[q_{i} \otimes_{S} p_{i} \cdot t\right]_{i}=\left[q_{i} \otimes_{S}\left[q_{j}\left(p_{i} \cdot t\right) \cdot p_{j}\right]_{j}\right]_{i}=\left[q_{i} \cdot q_{j}\left(p_{i} \cdot t\right) \otimes_{S} p_{j}\right]_{i, j}} \\
=\left[\left[q_{i} \cdot q_{j}\left(p_{i} \cdot t\right)\right]_{i} \otimes_{S} p_{j}\right]_{j} \stackrel{(*)}{=}\left[t \cdot q_{j} \otimes_{S} p_{j}\right]_{j}=t \cdot\left[q_{j} \otimes_{S} p_{j}\right]_{j},
\end{gathered}
$$

where $(*)$ follows from the fact that ev is a $T$-balanced map. Whence $\mathrm{db}$ is a $T$-bimodule homomorphism. On the other hand,

$$
\mathrm{ev}\left(p \otimes_{T} q \cdot s\right)=\varepsilon_{S}\left(p \otimes_{T} q \otimes_{S} \rho_{s}\left(1_{S}\right)\right) \stackrel{\text { 7.1.4 }}{=} \mathrm{ev}\left(p \otimes_{T} q\right) s
$$

and hence ev is an $S$-bimodule homomorphism. In view of this, 7.1.5 can now be rewritten as 7.1.3.

Conversely, to prove that 2 implies 1 consider the functors $P \otimes_{T}-: T$-mod $\longrightarrow$ $S$-mod and $Q \otimes_{S}-: S$-mod $\longrightarrow T$-mod. If we define unit and counit by

$$
\begin{gathered}
\eta_{M}:=\mathrm{db} \otimes_{T} M: M \longrightarrow Q \otimes_{S} P \otimes_{T} M, \\
\varepsilon_{N}:=\mathrm{ev} \otimes_{S} N: P \otimes_{T} Q \otimes_{S} N \longrightarrow N,
\end{gathered}
$$


for every $T$-module $M$ and every $S$-module $N$, then the zigzag identities (7.1.3) entail that $P \otimes_{T}-$ is left adjoint to $Q \otimes_{S}-$ and the fact that $\eta$ and $\varepsilon$ are natural isomorphisms implies in addition that these two functors define an equivalence of categories.

Remark 7.1.6. By checking closely the proof of Theorem 7.1.5, one may notice that $\mathrm{R} \cong \operatorname{Hom}_{S}(P,-)$ as the right adjoint functor of $\mathrm{L} \cong P \otimes_{T}-$, and $\mathrm{R} \cong Q \otimes_{S}-$ since it is a left adjoint functor itself. Therefore,

$$
{ }^{*} P:=\operatorname{Hom}_{S}\left({ }_{S} P, S\right) \cong \mathrm{R}(S) \cong Q \otimes_{S} S \cong Q
$$

as $T$-S-bimodules. Analogously, $P \cong Q^{*}:=\operatorname{Hom}_{S}\left(Q_{S}, S\right)$ as $S$-T-bimodules. Moreover, we point out that any argument provided for left modules would hold symmetrically for right modules.

A distinguished functor $\mathrm{F}: S$ - $\bmod \longrightarrow T$-mod is the restriction of scalars functor $\mathrm{F}=f^{*}$ associated with a truss homomorphism $f: T \longrightarrow S$. This is the faithful functor sending every left $S$-module $M$ to the left $T$-module ${ }_{f} M:=f^{*}(M)$ having the same underlying heap structure but action given by $t \cdot m=f(t) \cdot m$ for all $t \in T, m \in M$, and sending every $S$-linear morphism to itself, but now seen as a $T$-linear map. We already saw examples of restriction of scalars functors in Theorem 6.1.2 and Proposition 6.3.13 (the forgetful functor $\mathrm{U}_{\mathbf{m o d}}: T_{1}$-mod $\longrightarrow \mathbf{A h}$ can be seen as a restriction of scalars along the unital truss homomorphism $\eta:\{1\} \longrightarrow T)$.

Proposition 7.1.7. The restriction of scalars functor $\mathrm{F}: S$ - $\bmod \longrightarrow T$-mod associated with a truss homomorphism $f: T \longrightarrow S$ satisfies

$$
\operatorname{Hom}_{S}\left(\left(S_{u}\right)_{f},-\right) \cong \mathrm{F} \cong{ }_{f}\left(S_{u}\right) \otimes_{S}-
$$

In particular, there is an adjoint triple of functors:

$$
\left(S_{u}\right)_{f} \otimes_{T}-\dashv \mathrm{F} \dashv \operatorname{Hom}_{T}\left(f\left(S_{u}\right),-\right) .
$$


Proof. For every left $S$-module $M$, consider the assignments

$$
\begin{array}{r}
\operatorname{Hom}_{S}\left(\left(S_{u}\right)_{f}, M\right) \\
\phi \longmapsto{ }_{f} M \\
{\left[\begin{array}{c}
1 \longmapsto \\
s \longmapsto
\end{array}\right]}
\end{array}
$$

$$
\begin{gathered}
f M \longleftrightarrow{ }_{f}\left(S_{u}\right) \otimes_{S} M \\
m \longmapsto 1 \otimes_{S} m \\
{\left[z_{i} \cdot m_{i}\right]_{i=1}^{2 k+1} \longleftrightarrow\left[z_{i} \otimes_{S} m_{i}\right]_{i=1}^{2 k+1}}
\end{gathered}
$$

as in the proof of Proposition 6.3.7. They are $T$-linear isomorphisms, natural in $M$.

Let $T$ be a truss. The conditions in Theorem 7.1.5 and the subsequent observations in Remark 7.1.6 call for a closer analysis of $T$-modules admitting a dual basis db and evaluation ev morphisms.

Definition 7.1.8. A module $P$ over a truss $T$ is said to satisfy the dual basis property (DBP for short) if there exist an odd integer $s=2 k+1$, an element $\left(e_{1}, \ldots, e_{s}\right) \in P^{s}$ and an element $\left(\phi_{1}, \ldots, \phi_{s}\right) \in \operatorname{Hom}_{T}(P, T)^{s}$ such that, for all $p \in P$,

$$
p=\left[\phi_{1}(p) \cdot e_{1}, \ldots, \phi_{s}(p) \cdot e_{s}\right]
$$

We call the pair $\left\{\left(e_{1}, \ldots, e_{s}\right),\left(\phi_{1}, \ldots, \phi_{s}\right)\right\}$ a dual basis for $P$.

\section{Example 7.1.9.}

1. The empty $T$-module $\varnothing$ never satisfies the DBP.

2. If $T$ is unital, then $P=T$ itself satisfies the DBP with $e_{1}=1_{T}$ and $\phi_{1}=1_{T}$.

3. The singleton $T$-module $\{1\}$ satisfies the DPB if and only if $T$ admits a left absorber. Indeed, if $T$ admits a left absorber 0 then $\{1\}$ satisfies the DBP with $e_{1}=1$ and $\phi_{1}:\{1\} \longrightarrow T, 1 \longmapsto 0$. Conversely, if $\{1\}$ satisfies the DBP then $\phi_{1}(1) \in T$ is a left absorber.

4. If $T$ is a unital truss with identity $1_{T}$ and $S$ is a truss with a left absorber $a$, then $T$ satisfies the DBP as an $S \times T$-module with $e_{1}=1_{T}$ and $\phi_{1}: T \longrightarrow S \times T, t \longmapsto$ $(a, t)$. For example, if we take $S=E(T)^{o p}$ with $a: T \longrightarrow T, t \longmapsto 1_{T}$, then $T$ satisfies the DBP as an $\left(E(T)^{o p} \times T\right)$-module. 
As usual, let $T_{u}$ be the unital extension of $T$. Set ${ }^{*} P:=\operatorname{Hom}_{T}\left(P, T_{u}\right)$. It is a right $T$-module with $(f \cdot t)(p):=f(p) t$ for all $f \in{ }^{*} P, t \in T$ and $p \in P$.

Remark 7.1.10.

1. If $P$ satisfies the DBP, then ${ }^{*} P$ satisfies the DBP. For every $i=1, \ldots, s$, consider the right $T$-linear morphism

$$
\mathrm{ev}_{i}:{ }^{*} P \longrightarrow T_{u}, \quad \alpha \longmapsto \alpha\left(e_{i}\right)
$$

Then, for all $\alpha \in{ }^{*} P$,

$$
\alpha(p)=\alpha\left(\left[\phi_{k}(p) \cdot e_{k}\right]_{k=1}^{s}\right)=\left[\phi_{k}(p) \alpha\left(e_{k}\right)\right]_{k=1}^{s}=\left[\phi_{k} \cdot \mathrm{ev}_{k}(\alpha)\right]_{k=1}^{s}(p)
$$

for all $p \in P$, whence $\alpha=\left[\phi_{k} \cdot \mathrm{ev}_{k}(\alpha)\right]_{k=1}^{s}$.

2. If $P$ satisfies the DBP, then for every $T$-module $M$ and for every $f: P \longrightarrow M$,

$$
f=\left[\phi_{k} f\left(e_{k}\right)\right]_{k=1}^{s}
$$

in $\operatorname{Hom}_{T}(P, M)$, where $\phi_{k} f\left(e_{k}\right): P \longrightarrow M, p \longmapsto \phi_{k}(p) f\left(e_{k}\right)$.

Theorem 7.1.11. Let $T$ be a truss and $P$ be a left $T$-module. The following properties are equivalent

1. The functor $\operatorname{Hom}_{T}(P,-): T-\mathbf{m o d} \longrightarrow \mathbf{A h}$ is right exact (that is, it preserves finite colimits) and $P$ is finitely generated.

2. The module P satisfies the DBP.

3. There exist a T-bilinear morphism ev $: P \otimes{ }^{*} P \longrightarrow T_{u}$ and a morphism of Abelian heaps $\mathrm{db}:\{1\} \longrightarrow{ }^{*} P \otimes_{T} P$ (that is, a $\{1\}$-bilinear morphism) such that

$$
\left(\mathrm{ev} \otimes_{T} P\right) \circ(P \otimes \mathrm{db})=1_{P} \quad \text { and } \quad\left({ }^{*} P \otimes_{T} \mathrm{ev}\right) \circ\left(\mathrm{db} \otimes{ }^{*} P\right)=1{ }^{*} P
$$

up to the canonical isomorphisms

$$
P \otimes\{1\} \cong P \cong T_{u} \otimes_{T} P, \quad{ }^{*} P \otimes_{T} T_{u} \cong{ }^{*} P \cong\{1\} \otimes{ }^{*} P
$$


4. The functor $\operatorname{Hom}_{T}(P,-)$ is naturally isomorphic to the functor ${ }^{*} P \otimes_{T}-$.

5. The functor $\operatorname{Hom}_{T}(P,-)$ is cocontinuous (that is, it preserves small colimits).

Proof. 1 $\Rightarrow$ 2. Assume that the functor $\operatorname{Hom}_{T}(P,-)$ preserves finite colimits. Since $P$ is finitely generated, there exist a positive integer $r$ and a $T$-module epimorphism $\pi: \mathcal{T}^{\{1, \ldots, r\}} \longrightarrow P$. For the sake of clarity and brevity, we denote by $T_{i}$ the copy of $T_{u}$ in position $i$ and by $1_{i} \in T_{i}$ its unit, for $i=1, \ldots, r$. By Proposition 4.4.2, $\pi$ is a coequalizer and, by hypothesis,

$$
\operatorname{Hom}_{T}(P, \pi): \operatorname{Hom}_{T}\left(P, \mathcal{T}^{\{1, \ldots, r\}}\right) \longrightarrow \operatorname{Hom}_{T}(P, P)
$$

is a coequalizer of the corresponding morphisms, whence an epimorphism in particular. Choose a pre-image in $\operatorname{Hom}_{T}\left(P, \mathcal{T}^{\{1, \ldots, r\}}\right)$ of $1_{P}$ and call it $\sigma$; it satisfies $\pi \circ \sigma=1_{P}$.

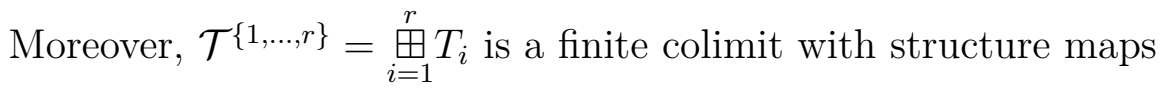

$$
\eta_{i}: T_{u} \longrightarrow \mathcal{T}^{\{1, \ldots, r\}}, \quad z \longmapsto z 1_{i} \in T_{i},
$$

for all $i=1, \ldots, r$. By hypothesis again, the induced morphism

$$
\underset{i=1}{\stackrel{r}{\boxplus}} \operatorname{Hom}_{T}\left(P, \eta_{i}\right): \stackrel{r}{\underset{i=1}{\boxplus}} \operatorname{Hom}_{T}\left(P, T_{u}\right)_{i} \longrightarrow \operatorname{Hom}_{T}\left(P, \mathcal{T}^{\{1, \ldots, r\}}\right)
$$

is an isomorphism, where $\operatorname{Hom}_{T}\left(P, T_{u}\right)_{i}$ denotes the copy of $\operatorname{Hom}_{T}\left(P, T_{u}\right)$ in position $i$. Therefore, there exist elements $\phi_{1}, \ldots, \phi_{s} \in \operatorname{Hom}_{T}\left(P, T_{u}\right)$ (possibly $r \neq s$ ) such that

$$
\left[\phi_{1}, \ldots, \phi_{s}\right] \in \underset{i=1}{\stackrel{r}{\boxplus}} \operatorname{Hom}_{T}\left(P, T_{u}\right)_{i}
$$

satisfies

$$
\underset{i=1}{\stackrel{r}{\boxplus}} \operatorname{Hom}_{T}\left(P, \eta_{i}\right)\left(\left[\phi_{1}, \ldots, \phi_{s}\right]\right)=\sigma .
$$

Concretely, this amounts to say that, for every $p \in P$,

$$
\begin{aligned}
p & =\pi(\sigma(p))=\pi\left(\left[\eta_{i_{1}} \circ \phi_{1}, \ldots, \eta_{i_{s}} \circ \phi_{s}\right](p)\right) \\
& =\left[\left(\pi \eta_{i_{1}} \phi_{1}\right)(p), \ldots,\left(\pi \eta_{i_{s}} \phi_{s}\right)(p)\right]=\left[\phi_{1}(p) \cdot \pi\left(1_{i_{1}}\right), \ldots, \phi_{s}(p) \cdot \pi\left(1_{i_{s}}\right)\right],
\end{aligned}
$$

where the $i_{k}$ are such that $\phi_{k} \in \operatorname{Hom}_{T}\left(P, T_{u}\right)_{i_{k}}, k=1, \ldots, s$. Set $e_{k}:=\pi\left(1_{i_{k}}\right) \in P$ for $k=1, \ldots, s$. The foregoing relation says that, for every $p \in P$,

$$
p=\left[\phi_{1}(p) \cdot e_{1}, \ldots, \phi_{s}(p) \cdot e_{s}\right] .
$$


We conclude that if $P$ is finitely generated and if $\operatorname{Hom}_{T}(P,-)$ preserves finite colimits, then $P$ satisfies the DBP.

$2 \Rightarrow 3$. Consider the assignment

$$
e: P \times{ }^{*} P \longrightarrow T_{u}, \quad(p, \alpha) \longmapsto \alpha(p)
$$

Then

$$
e\left(\left[p, p^{\prime}, p^{\prime \prime}\right], \alpha\right)=\alpha\left(\left[p, p^{\prime}, p^{\prime \prime}\right]\right)=\left[\alpha(p), \alpha\left(p^{\prime}\right), \alpha\left(p^{\prime \prime}\right)\right]=\left[e(p, \alpha), e\left(p^{\prime}, \alpha\right), e\left(p^{\prime \prime}, \alpha\right)\right]
$$

and

$$
e\left(p,\left[\alpha, \alpha^{\prime}, \alpha^{\prime \prime}\right]\right)=\left[\alpha, \alpha^{\prime}, \alpha^{\prime \prime}\right](p)=\left[\alpha(p), \alpha^{\prime}(p), \alpha^{\prime \prime}(p)\right]=\left[e(p, \alpha), e\left(p, \alpha^{\prime}\right), e\left(p, \alpha^{\prime \prime}\right)\right],
$$

whence there exists a unique heap homomorphism ev $: P \otimes{ }^{*} P \longrightarrow T_{u}$ such that $\mathrm{ev}(p \otimes \alpha)=\alpha(p)$, for all $p \in P, \alpha \in{ }^{*} P$. Moreover,

$$
\mathrm{ev}(t \cdot p \otimes \alpha)=\alpha(t \cdot p)=t \alpha(p)
$$

and

$$
\mathrm{ev}(p \otimes \alpha \cdot t)=(\alpha \cdot t)(p)=\alpha(p) t
$$

for all $p \in P, \alpha \in{ }^{*} P, t \in T$, whence ev is $T$-bilinear. Consider also the assignment

$$
\mathrm{db}:\{1\} \longrightarrow{ }^{*} P \otimes_{T} P, \quad * \longmapsto\left[\phi_{k} \otimes_{T} e_{k}\right]_{k=1}^{s}
$$

A direct check shows that

$$
\begin{gathered}
\left(\left(\mathrm{ev} \otimes_{T} P\right) \circ(P \otimes \mathrm{db})\right)(p)=\left(\mathrm{ev} \otimes_{T} P\right)\left(\left[p \otimes \phi_{k} \otimes_{T} e_{k}\right]_{k=1}^{s}\right)=\left[\phi_{k}(p) \cdot e_{k}\right]_{k=1}^{s}=p, \\
\left(\left({ }^{*} P \otimes_{T} \mathrm{ev}\right) \circ\left(\mathrm{db} \otimes{ }^{*} P\right)\right)(\alpha)=\left({ }^{*} P \otimes_{T} \text { ev }\right)\left(\left[\phi_{k} \otimes_{T} e_{k} \otimes \alpha\right]_{k=1}^{s}\right)=\left[\phi_{k} \cdot \alpha\left(e_{k}\right)\right]_{k=1}^{s}=\alpha,
\end{gathered}
$$

for all $p \in P, \alpha \in{ }^{*} P$.

3 $\Rightarrow$ 4. For every $T$-module $M$, consider

$$
\tilde{\tau}:{ }^{*} P \times M \longrightarrow \operatorname{Hom}_{T}(P, M), \quad(\alpha, m) \longmapsto[p \longmapsto \operatorname{ev}(p \otimes \alpha) \cdot m]
$$


For all $p \in P, \alpha, \alpha^{\prime}, \alpha^{\prime \prime} \in{ }^{*} P, m, m^{\prime}, m^{\prime \prime} \in M, t \in T$,

$$
\begin{aligned}
\tilde{\tau}\left(\left[\alpha, \alpha^{\prime}, \alpha^{\prime \prime}\right], m\right)(p) & =\left[\alpha, \alpha^{\prime}, \alpha^{\prime \prime}\right](p) \cdot m=\left[\alpha(p), \alpha^{\prime}(p), \alpha^{\prime \prime}(p)\right] \cdot m \\
& =\left[\alpha(p) \cdot m, \alpha^{\prime}(p) \cdot m, \alpha^{\prime \prime}(p) \cdot m\right] \\
& =\left[\tilde{\tau}(\alpha, m)(p), \tilde{\tau}\left(\alpha^{\prime}, m\right)(p), \tilde{\tau}\left(\alpha^{\prime \prime}, m\right)(p)\right] \\
& =\left[\tilde{\tau}(\alpha, m), \tilde{\tau}\left(\alpha^{\prime}, m\right), \tilde{\tau}\left(\alpha^{\prime \prime}, m\right)\right](p), \\
\tilde{\tau}\left(\alpha,\left[m, m^{\prime}, m^{\prime \prime}\right]\right)(p) & =\alpha(p) \cdot\left[m, m^{\prime}, m^{\prime \prime}\right]=\left[\alpha(p) \cdot m, \alpha(p) \cdot m^{\prime}, \alpha(p) \cdot m^{\prime \prime}\right] \\
& =\left[\tilde{\tau}(\alpha, m)(p), \tilde{\tau}\left(\alpha, m^{\prime}\right)(p), \tilde{\tau}\left(\alpha, m^{\prime \prime}\right)(p)\right] \\
& =\left[\tilde{\tau}(\alpha, m), \tilde{\tau}\left(\alpha, m^{\prime}\right), \tilde{\tau}\left(\alpha, m^{\prime \prime}\right)\right](p), \\
\tilde{\tau}(\alpha \cdot t, m)(p) & =(\alpha \cdot t)(p) \cdot m=\alpha(p) t \cdot m=\tilde{\tau}(\alpha, t \cdot m)(p) .
\end{aligned}
$$

Therefore, there exists a unique heap homomorphism $\tau_{M}:{ }^{*} P \otimes_{T} M \longrightarrow \operatorname{Hom}_{T}(P, M)$ such that $\tau\left(\alpha \otimes_{T} m\right): p \longmapsto \alpha(p) \cdot m$. The other way around, write explicitly $\mathrm{db}(1)=$ $\left[\phi_{k} \otimes_{T} e_{k}\right]_{k=1}^{s}$ and consider the assignment

$$
\sigma_{M}: \operatorname{Hom}_{T}(P, M) \longrightarrow{ }^{*} P \otimes_{T} M, \quad f \longmapsto\left({ }^{*} P \otimes_{T} f\right)(\mathrm{db}(1))=\left[\phi_{k} \otimes_{T} f\left(e_{k}\right)\right]_{k=1}^{s} .
$$

A direct computation shows that

$$
\sigma_{M} \tau_{M}\left(\alpha \otimes_{T} m\right)=\left[\phi_{k} \otimes_{T} \alpha\left(e_{k}\right) \cdot m\right]_{k=1}^{s}=\left[\phi_{k} \cdot \alpha\left(e_{k}\right)\right]_{k=1}^{s} \otimes_{T} m=\alpha \otimes_{T} m
$$

for all $m \in M, \alpha \in{ }^{*} P$, and

$$
\tau_{M} \sigma_{M}(f)(p)=\left[\phi_{k}(p) \cdot f\left(e_{k}\right)\right]_{k=1}^{s}=f
$$

whence they are inverses of each other. Furthermore, if $g: M \longrightarrow N$ is any $T$-linear map, then

$$
\tau_{N}\left({ }^{*} P \otimes_{T} g\right)\left(\alpha \otimes_{T} m\right)(p)=\alpha(p) g(m)=\left(\operatorname{Hom}_{T}(P, g) \circ \tau_{M}\right)\left(\alpha \otimes_{T} m\right)(p),
$$

for all $p \in P, m \in M, \alpha \in{ }^{*} P$, so that $\tau$ is also natural in $M$.

$4 \Rightarrow 5$. Obvious, since tensoring by a right $T$-module is a left adjoint.

$5 \Rightarrow$ 1. Clearly, $\operatorname{Hom}_{T}(P,-)$ is a right exact functor. Thus, we are left to show that $P$ has to be finitely generated. Since $P$ is a set, we can consider the epimorphism 
$\pi: \mathcal{T}^{P} \longrightarrow P$ uniquely determined by the assignments $T_{p} \rightarrow P, z \longmapsto z \cdot p$, for all $p \in P$. Since epimorphisms are coequalizers, $\pi_{*}: \operatorname{Hom}_{T}\left(P, \mathcal{T}^{P}\right) \longrightarrow \operatorname{Hom}_{T}(P, P), \psi \longmapsto \pi \circ \psi$, is still a coequalizer (whence an epimorphism), and since $\mathcal{T}^{P}$ is a small coproduct,

$$
\operatorname{Hom}_{T}\left(P, \mathcal{T}^{P}\right) \cong \underset{p \in P}{\boxplus} \operatorname{Hom}_{T}\left(P, T_{u}\right)_{p}
$$

As in the proof of 1 2, one can consider a pre-image of $1_{P}$ via $\pi_{*}$ and call it $\sigma$. There exist elements $\phi_{1}, \ldots, \phi_{s} \in \operatorname{Hom}_{T}\left(P, T_{u}\right)$ such that

$$
\left[\phi_{1}, \ldots, \phi_{s}\right] \in \underset{p \in P}{\boxplus} \operatorname{Hom}_{T}\left(P, T_{u}\right)_{p}
$$

satisfies

$$
\underset{p \in P}{\boxplus} \operatorname{Hom}_{T}\left(P, \eta_{p}\right)\left(\left[\phi_{1}, \ldots, \phi_{s}\right]\right)=\sigma .
$$

Concretely, this amounts to say that, for every $q \in P$,

$$
\begin{aligned}
q & =\pi(\sigma(q))=\pi\left(\left[\eta_{p_{1}} \circ \phi_{1}, \ldots, \eta_{p_{s}} \circ \phi_{s}\right](q)\right) \\
& =\left[\left(\pi \eta_{p_{1}} \phi_{1}\right)(q), \ldots,\left(\pi \eta_{p_{s}} \phi_{s}\right)(q)\right]=\left[\phi_{1}(q) \cdot \pi\left(1_{p_{1}}\right), \ldots, \phi_{s}(q) \cdot \pi\left(1_{p_{s}}\right)\right] .
\end{aligned}
$$

Set $e_{k}:=\pi\left(1_{p_{k}}\right) \in P$ for $k=1, \ldots, s$. Since the foregoing relation says that for every $p \in P, p=\left[\phi_{1}(p) \cdot e_{1}, \ldots, \phi_{s}(p) \cdot e_{s}\right]$, we conclude that the $e_{k}$ form a finite family of generators of $P$.

By taking inspiration from [37, §5.5] and in light of Theorem 7.1.11, we give the following definition (see also [38, $\S 3]$ ).

Definition 7.1.12. A $T$-module $P$ satisfying the equivalent conditions of Theorem 7.1.11 is called tiny (or small-projective).

Remark 7.1.13.

1. In the proof of the implication $1 \Rightarrow 2$ in Theorem 7.1 .11 , there is no need for $s$ to be exactly $r$.

2. The dual basis map db does not depend on the choice of the dual basis. In fact, if $\left\{\left(e_{1}, \ldots, e_{s}\right),\left(\phi_{1}, \ldots, \phi_{s}\right)\right\}$ and $\left\{\left(f_{1}, \ldots, f_{r}\right),\left(\psi_{1}, \ldots, \psi_{r}\right)\right\}$ are two dual bases, then

$$
\begin{aligned}
{\left[\phi_{k} \otimes_{T} e_{k}\right]_{k=1}^{s} } & =\left[\phi_{k} \otimes_{T}\left[\psi_{h}\left(e_{k}\right) \cdot f_{h}\right]_{h=1}^{r}\right]_{k=1}^{s} \stackrel{1.1 .2}{-}\left[\left[\phi_{k} \cdot \psi_{h}\left(e_{k}\right) \otimes_{T} f_{h}\right]_{k=1}^{s}\right]_{h=1}^{r} \\
& =\left[\left[\phi_{k} \cdot \psi_{h}\left(e_{k}\right)\right]_{k=1}^{s} \otimes_{T} f_{h}\right]_{h=1}^{r}=\left[\psi_{h} \otimes_{T} f_{h}\right]_{h=1}^{r} .
\end{aligned}
$$


3. The implication from 5 to 4 in Theorem 7.1.11 follows also from the EilenbergWatts theorem, since $\operatorname{Hom}_{T}(P,-)$ is a heap functor.

4. In the implication from 4 to 3 in Theorem 7.1.11, the dual basis map $\mathrm{db}$ corresponds to the image of the identity morphism $1_{P}$ via the isomorphism $\operatorname{Hom}_{T}(P, P) \cong$ ${ }^{*} P \otimes_{T} P$.

5. In the present section, we always worked with a left $T$-module $P$, implicitly viewed as a $T$-\{1\}-bimodule. Observe that there is nothing particular in considering the distinguished truss $\{1\}$ instead of any other truss. Therefore, the description and the properties of a small-projective $T$-module developed so far can be adapted, with no additional effort, to speak about a $T$-S-bimodule which is small-projective over $T$ on the left.

Example 7.1.14. If $P$ is a finitely generated and projective module over a ring $R$, then $\mathrm{T}(P)$ is a tiny $\mathrm{T}(R)$-module.

Example 7.1.15 (Free modules are not tiny). Let $T$ be a unital truss and consider the free $T$-module $T \boxplus T$. Assume, by contradiction, that $T \boxplus T$ admits a dual basis $\left\{\left(e_{1}, \ldots, e_{s}\right),\left(\phi_{1}, \ldots, \phi_{s}\right)\right\}$. Denote by $a=1_{T}$ the unit of the left-hand side copy of $T$ and by $b=1_{T}$ the one of the right-hand side copy. Taking advantage of the heap isomorphism in Proposition 1.4.4, we may construct the heap homomorphism that "measures tails"

$$
\ell: T \boxplus T \cong \mathrm{H}(\mathrm{G}(T, a) \oplus \mathrm{G}(T, b) \oplus \mathbb{Z}) \longrightarrow \mathrm{H}(\mathbb{Z}) .
$$

Notice that, being composition of heap homomorphisms, $\ell$ is not influenced by the reduction of a symmetric word $w$ to one of the "canonical forms" $t, s, t s b$, sta, tsab $\cdots b a$, $s t b a \cdots a b$. Therefore, the "length of tails" is well-defined and, in particular, it is not influenced by the action of $T$, see 4.3.1 . Summing up, for all $z \in T \boxplus T$

$$
\ell\left(\left[\phi_{1}(z) \cdot e_{1}, \ldots, \phi_{s}(z) \cdot e_{s}\right]\right)=\left[\ell\left(\phi_{1}(z) \cdot e_{1}\right), \ldots, \ell\left(\phi_{s}(z) \cdot e_{s}\right)\right]=\left[\ell\left(e_{1}\right), \ldots, \ell\left(e_{s}\right)\right] .
$$

However, if we set $m:=\left[\ell\left(e_{1}\right), \ldots, \ell\left(e_{s}\right)\right]$ (which does not depend on $z$ ) and we consider $z:=b a b \cdots a b$ with $|m|+1$ instances of $b$, then $\ell(z)=|m|+1$, which is a contradiction. 
Example 7.1.16. Let $T$ be a unital truss admitting a left absorber $a \in T$ and consider $P:=T \times T \times T$. Set

$$
\begin{array}{rlrl}
e_{1}:=\left(1_{T}, a, a\right), & e_{2}:=\left(a, 1_{T}, a\right), & e_{3}=\left(a, a, 1_{T}\right) \quad \text { and } \\
\phi_{1}: T \times T \times T \longrightarrow T, & (x, y, z) \longmapsto x, \\
\phi_{2}: T \times T \times T \longrightarrow T, & (x, y, z) \longmapsto[a, y, a], \\
\phi_{3}: T \times T \times T \longrightarrow T, & (x, y, z) \longmapsto z .
\end{array}
$$

Then these form a dual basis for $P$ as a left $T$-module.

Assume furthermore that $a$ is a two-sided absorber. Denote by $S$ the set of all $3 \times 3$ matrices with coefficients in $T$. They inherits an Abelian heap structure from the identification $S=T^{9}$ (that is, the bracket is taken component-wise). Moreover, $S$ admits a truss structure with the row-by-column multiplication

$$
\left(\begin{array}{lll}
t_{1,1} & t_{1,2} & t_{1,3} \\
t_{2,1} & t_{2,2} & t_{2,3} \\
t_{3,1} & t_{3,2} & t_{3,3}
\end{array}\right) \cdot\left(\begin{array}{lll}
s_{1,1} & s_{1,2} & s_{1,3} \\
s_{2,1} & s_{2,2} & s_{2,3} \\
s_{3,1} & s_{3,2} & s_{3,3}
\end{array}\right)=\left(r_{i, j}\right) \quad \text { where } \quad r_{i, j}=\left[t_{i, 1} s_{1, j}, t_{i, 2} s_{2, j}, t_{i, 3} s_{3, j}\right] .
$$

As for matrices over rings, $P$ becomes a right $S$-module with row-by-column action

$$
\left(\begin{array}{lll}
x & y & z
\end{array}\right)\left(\begin{array}{lll}
t_{1,1} & t_{1,2} & t_{1,3} \\
t_{2,1} & t_{2,2} & t_{2,3} \\
t_{3,1} & t_{3,2} & t_{3,3}
\end{array}\right)=\left(\left[x t_{1,1}, y t_{2,1}, z t_{3,1}\right] \quad\left[x t_{1,2}, y t_{2,2}, z t_{3,2}\right] \quad\left[x t_{1,3}, y t_{2,3}, z t_{3,3}\right]\right)
$$

which makes of it a $T$-S-bimodule and

$$
Q=\left\{\left(\begin{array}{l}
x \\
y \\
z
\end{array}\right) \mid x, y, z \in T\right\}
$$

becomes a $S$-T-bimodule analogously. Define the following morphisms

$$
\begin{gathered}
\text { ev }: Q \otimes_{T} P \longrightarrow S, \quad\left(\begin{array}{l}
x \\
y \\
z
\end{array}\right) \otimes_{T}\left(\begin{array}{lll}
x^{\prime} & y^{\prime} & z^{\prime}
\end{array}\right) \longmapsto\left(\begin{array}{l}
x \\
y \\
z
\end{array}\right) \cdot\left(\begin{array}{lll}
x^{\prime} & y^{\prime} & z^{\prime}
\end{array}\right)=\left(\begin{array}{lll}
x x^{\prime} & x y^{\prime} & x z^{\prime} \\
y x^{\prime} & y y^{\prime} & y z^{\prime} \\
z x^{\prime} & z y^{\prime} & z z^{\prime}
\end{array}\right) \\
\mathrm{db}: T \longrightarrow P \otimes_{S} Q, \quad 1_{T} \longmapsto\left(\begin{array}{lll}
1_{T} & a & a
\end{array}\right) \otimes_{S}\left(\begin{array}{c}
1_{T} \\
a \\
a
\end{array}\right) .
\end{gathered}
$$

They are invertible with inverses explicitly given by 


$$
\begin{aligned}
& \mathrm{ev}^{-1}:\left(t_{i, j}\right) \longmapsto\left[\left(\begin{array}{c}
t_{1,1} \\
t_{1,2} \\
t_{1,3}
\end{array}\right) \otimes_{T}\left(\begin{array}{lll}
1_{T} & a & a
\end{array}\right),\left(\begin{array}{c}
t_{2,1} \\
t_{2,2} \\
t_{2,3}
\end{array}\right) \otimes_{T}\left(\begin{array}{lll}
a & {\left[a, 1_{T}, a\right.}
\end{array}\right]\right. \\
& \text { and } \quad \mathrm{db}^{-1}:\left(\begin{array}{lll}
x & y & z
\end{array}\right) \otimes_{S}\left(\begin{array}{l}
x^{\prime} \\
y^{\prime} \\
z^{\prime}
\end{array}\right) \longmapsto\left(\begin{array}{lll}
x & y & z
\end{array}\right) \cdot\left(\begin{array}{l}
x^{\prime} \\
y^{\prime} \\
z^{\prime}
\end{array}\right)=\left[\begin{array}{ll}
x x^{\prime}, y y^{\prime}, z z^{\prime}
\end{array}\right] .
\end{aligned}
$$

Therefore, $T$-mod is equivalent to $S$-mod by Theorem 7.1 .5 .

\subsection{Projective modules over trusses}

Let $T$ be a truss (not necessarily unital) and let $\{0\}$ denote the singleton $T$-module. Recall from the Definition 4.2 .2 that if $(M, \cdot)$ is a non-empty $T$-module and $e \in M$, then we denote by $M^{(e)}=\left(M, \triangleright_{e}\right)$ the $T$-module with the induced action

$$
t \triangleright_{e} m=[t \cdot m, t \cdot e, e] .
$$

We say that a sequence of non-empty $T$-modules $M \stackrel{f}{\longrightarrow} N \stackrel{g}{\longrightarrow} P$ is exact provided there exists $e \in \operatorname{Im}(g)$ such that $\operatorname{Im}(f)=\operatorname{ker}_{e}(g)$ as sets. Notice that, in this case, $\operatorname{Im}(f) \cong \operatorname{ker}_{e^{\prime}}(g)$ as induced submodules for any other $e^{\prime} \in \operatorname{Im}(g)$.

Lemma 7.2.1. Let $M, N, P$ be T-modules and $f: M \longrightarrow N$ and $g: N \longrightarrow P$ be T-linear maps. There exist exact sequences

$$
M \stackrel{f}{\longrightarrow} N \stackrel{g}{\longrightarrow} P, \quad\{0\} \longrightarrow M^{(e)} \stackrel{f}{\longrightarrow} N^{(f(e))} \text { and } N \stackrel{g}{\longrightarrow} P \longrightarrow\{0\}
$$

if and only if

(a) $f$ is injective and

(b) $N / \operatorname{Im}(f) \cong P$ as $T$-modules,

where the module structure on $N / \operatorname{Im}(f)$ is the one for which the canonical projection $\pi: N \longrightarrow N / \operatorname{Im}(f)$ is $T$-linear.

Proof. Assume the sequences are exact. Then $f$ is injective, by the exactness of the second sequence, and

$$
P=\operatorname{Im}(g) \cong N / \operatorname{Ker}(g) \cong N / \operatorname{Im}(f),
$$


where the equality is a restatement of the third sequence, the second isomorphism follows by the exactness of the first sequence, and the first one is simply the first isomorphism theorem for $T$-modules.

Conversely, assume that there is an isomorphism $h: N / \operatorname{Im}(f) \longrightarrow P$ of $T$-modules, and denote by $\pi: N \longrightarrow N / \operatorname{Im}(f)$ the quotient map. The sub-heap $\operatorname{Im}(f)$ of $N$, as a kernel of $\pi$, admits an additional induced submodule structure. Denote it by $\operatorname{Im}(f)^{(e)} \subseteq N^{(e)}$ for a certain $e \in \operatorname{Im}(f)$. This entails that $\operatorname{Im}(f)$ is a submodule of $N$ with respect to two (in principle, different) $T$-modules structures: $\operatorname{Im}(f) \subseteq N$ with respect to the $T$-action for which $f$ is $T$-linear and $\operatorname{Im}(f)^{(e)} \subseteq N^{(e)}$ with respect to the induced action coming from the identification $\operatorname{Im}(f)=\operatorname{ker}_{\pi(e)}(\pi)$. Since $f$ is injective, we may transport the induced module structure on $M$. Denote it by $M^{\left(e^{\prime}\right)}$ for $e^{\prime} \in M$ such that $f\left(e^{\prime}\right)=e$. Consider the sequences

$$
\{0\} \longrightarrow M^{\left(e^{\prime}\right)} \stackrel{f}{\longrightarrow} N^{(e)}, \quad M \stackrel{f}{\longrightarrow} N \stackrel{h \circ \pi}{\longrightarrow} P, \quad N \stackrel{h \circ \pi}{\longrightarrow} P \longrightarrow\{0\} .
$$

They are exact.

By abuse of notation, we will say that

$$
\{0\} \cdots \cdots \cdots, \stackrel{f}{\longrightarrow} \geq N \stackrel{g}{\longrightarrow} P \longrightarrow\{0\}
$$

is a short exact sequence of $T$-modules to mean that there exists $e \in M$ such that all three sequences (7.2.1) are exact.

Proposition 7.2.2. Let $\phi: M \longrightarrow N$ and $\psi: N \longrightarrow P$ be morphisms of T-modules. Assume that $\psi$ is surjective, that $\phi$ admits a retraction $\gamma$ (in particular, it is injective) and that

$$
M \stackrel{\phi}{\longrightarrow} N \stackrel{\psi}{\longrightarrow} P
$$

is exact. Then $N \cong M \times P$ as T-modules. We will call such a sequence a split exact sequence.

Proof. Since (7.2.2) is exact, there exists $e \in P$ such that $\operatorname{ker}_{e}(\psi)=\operatorname{Im}(\phi)$. Consider $e^{\prime} \in N$ such that $\psi\left(e^{\prime}\right)=e$ and consider $\gamma\left(e^{\prime}\right) \in M$. Since $e^{\prime} \in \operatorname{ker}_{e}(\psi)=\operatorname{Im}(\phi)$, 
$\phi\left(\gamma\left(e^{\prime}\right)\right)=e^{\prime}$. Denote by $\mathrm{G}(P ; e), \mathrm{G}\left(M ; \gamma\left(e^{\prime}\right)\right)$ and $\mathrm{G}\left(N ; e^{\prime}\right)$ the retracts of the heaps $P$, $M$ and $N$ respectively. Observe that map $\phi$ induces an additive map of retracts,

$$
\widehat{\phi}: \mathrm{G}\left(M ; \gamma\left(e^{\prime}\right)\right) \longrightarrow \mathrm{G}\left(N ; e^{\prime}\right), \quad m \longmapsto\left[\phi(m), \phi \gamma\left(e^{\prime}\right), e^{\prime}\right]=\left[\phi(m), e^{\prime}, e^{\prime}\right]=\phi(m),
$$

and, analogously, $\widehat{\psi}=\psi$ and $\widehat{\gamma}=\gamma$, which entail that

$$
0 \longrightarrow \mathrm{G}\left(M ; \gamma(\underbrace{\stackrel{\phi}{\longrightarrow}}_{\gamma} \mathrm{G}\left(N ; e^{\prime}\right) \stackrel{\psi}{\longrightarrow} \mathrm{G}(P ; e) \longrightarrow 0\right.
$$

is a split short exact sequence of $\mathbb{Z}$-modules. Thus,

$$
\mathrm{G}\left(N ; e^{\prime}\right) \cong \mathrm{G}\left(M ; \gamma\left(e^{\prime}\right)\right) \oplus \mathrm{G}(P ; e) \cong \mathrm{G}\left(M ; \gamma\left(e^{\prime}\right)\right) \times \mathrm{G}(P ; e) .
$$

From $\mathrm{G}\left(N ; e^{\prime}\right) \cong \mathrm{G}\left(M ; \gamma\left(e^{\prime}\right)\right) \times \mathrm{G}(P ; e)$ and Corollary 1.1.29, it follows that

$$
\begin{aligned}
N=\mathrm{H}\left(\mathrm{G}\left(N ; e^{\prime}\right)\right) & \cong \mathrm{H}\left(\mathrm{G}\left(M ; \gamma\left(e^{\prime}\right)\right) \times \mathrm{G}(P ; e)\right) \\
& \cong \mathrm{H}\left(\mathrm{G}\left(M ; \gamma\left(e^{\prime}\right)\right)\right) \times \mathrm{H}(\mathrm{G}(P ; e))=M \times P .
\end{aligned}
$$

Summing up, at the heap level there is a (unique) isomorphism $N \cong M \times P$ induced by the universal property of the product and explicitly given by

$$
\Phi: N \longrightarrow M \times P, \quad n \longmapsto(\gamma(n), \psi(n)) .
$$

By $T$-linearity of $\gamma$ and $\psi, \Phi$ is $T$-linear as well.

For the sake of completeness, we point out that the inverse of $\Phi$ is explicitly given by

$$
\Phi^{-1}: M \times P \longrightarrow N, \quad(m, p) \longmapsto\left[n_{p}, \phi \gamma\left(n_{p}\right), \phi(m)\right]
$$

where $n_{p} \in N$ is any element such that $\psi\left(n_{p}\right)=p$.

Corollary 7.2.3. Let $T$ be a truss and $n \in \mathbb{N}$. Then for any $k \leq n$ there exists a $T$-module with absorber $M$ such that $T^{k} \times M \cong T^{n}$.

Proof. Observe that $\phi: T^{k} \rightarrow T^{n}$ given by $\left(t_{1}, \ldots, t_{k}\right) \longmapsto\left(t_{1}, \ldots, t_{k}, t_{k}, \ldots, t_{k}\right)$ is a $T$-module homomorphism and clearly the sequence

$$
T^{k} \underset{\pi_{k}}{\longleftarrow} T^{n} \stackrel{\pi_{\operatorname{Im}(\phi)}}{\longrightarrow} T^{n} / \operatorname{Im}(\phi),
$$


where $\pi_{k}$ is the projection on the first $k$ coordinates, is a split exact sequence. Therefore by Proposition 7.2.2. $T^{n} \cong T^{k} \times\left(T^{n} / \operatorname{Im}(\phi)\right)$ and $T^{n} / \operatorname{Im}(\phi)$ is the requested module with an absorber.

Example 7.2.4. Let $T=2 \mathbb{Z}+1$ and let us consider $(2 \mathbb{Z}+1)^{3} \cong(2 \mathbb{Z}+1) \times M$ for some $(2 \mathbb{Z}+1)$-module $M$ as in Corollary 7.2.3. In this case, $\phi$ is the map given by $2 k+1 \longmapsto(2 k+1,2 k+1,2 k+1)$ for all $k \in \mathbb{Z}$. It is easy to check that $\mathrm{H}(M) \cong$ $\mathrm{H}((2 \mathbb{Z}+1) \times(2 \mathbb{Z}+1))$ and that the heap isomorphism is a $(2 \mathbb{Z}+1)$-module homomorphism for the $(2 \mathbb{Z}+1)$-action given on $(2 \mathbb{Z}+1) \times(2 \mathbb{Z}+1)$ by

$$
(2 k+1) \cdot(2 l+1,2 h+1)=(2(2 k+1) l+1,2(2 k+1) h+1),
$$

for all $k, l, h \in \mathbb{Z}$. The desired absorber is $(1,1)$.

Proposition 7.2.5. Let $\phi: M \longrightarrow N$ and $\psi: N \longrightarrow P$ be morphisms of $T$-modules. Assume that $\phi$ is injective, that $\psi$ admits a section $\sigma$ (in particular, it is surjective) and that

$$
M \stackrel{\phi}{\longrightarrow} N \stackrel{\psi}{\longrightarrow} P
$$

is exact. Then there exists $e^{\prime} \in M$ yielding an isomorphism of $T$-modules $N \cong M^{\left(e^{\prime}\right)} \times P$, where $M^{\left(e^{\prime}\right)}$ denotes the $e^{\prime}$-induced left T-module structure on $M$.

Proof. The argument for this proof follows closely that in the proof of Proposition 7.2.2. Since (7.2.3) is exact, there exists $e \in P$ such that $\operatorname{ker}_{e}(\psi)=\operatorname{Im}(\phi)$. Consider $\sigma(e) \in N$ and let $e^{\prime} \in M$ be the unique element such that

$$
\phi\left(e^{\prime}\right)=\sigma(e)
$$

Similarly to before, the heap homomorphism $\sigma$ induces an additive map of retracts

$$
\widehat{\sigma}: \mathrm{G}(P ; e) \longrightarrow \mathrm{G}(N ; \sigma(e)), \quad p \longmapsto[\sigma(p), \sigma(e), \sigma(e)]=\sigma(p)
$$

and analogously for $\phi$ and $\psi$. These yield the following split short exact sequence of $\mathbb{Z}$-modules

$$
0 \longrightarrow \mathrm{G}\left(M ; e^{\prime}\right) \stackrel{\phi}{\longrightarrow} \mathrm{G}(N ; \underbrace{\sigma(e)) \stackrel{\psi}{\longrightarrow}}_{\sigma} \mathrm{G}(P ; e) \longrightarrow 0 .
$$


Thus,

$$
\mathrm{G}(N ; \sigma(e)) \cong \mathrm{G}\left(M ; e^{\prime}\right) \oplus \mathrm{G}(P ; e) \cong \mathrm{G}\left(M ; e^{\prime}\right) \times \mathrm{G}(P ; e)
$$

From $\mathrm{G}(N ; \sigma(e)) \cong \mathrm{G}\left(M ; e^{\prime}\right) \times \mathrm{G}(P ; e)$ and Corollary 1.1.29, it follows that

$$
N=\mathrm{H}(\mathrm{G}(N ; \sigma(e))) \cong \mathrm{H}\left(\mathrm{G}\left(M ; e^{\prime}\right)\right) \times \mathrm{H}(\mathrm{G}(P ; e))=M \times P .
$$

Explicitly, this isomorphism is given by the rule

$$
\Theta(m, p)=[\phi(m), \sigma(e), \sigma(p)]
$$

Now, consider $M$ as a $T$-module with the induced structure $t \triangleright_{e^{\prime}} m=\left[t \cdot m, t \cdot e^{\prime}, e^{\prime}\right]$. Then

$$
\begin{gathered}
\Theta\left(t \triangleright_{e^{\prime}} m, t \cdot p\right)=\Theta\left(\left[t \cdot m, t \cdot e^{\prime}, e^{\prime}\right], t \cdot p\right)=\left[\phi(t \cdot m), \phi\left(t \cdot e^{\prime}\right), \phi\left(e^{\prime}\right), \sigma(e), \sigma(t \cdot p)\right] \\
\stackrel{\text { 7.2.4 }}{=}\left[t \cdot \phi(m), t \cdot \phi\left(e^{\prime}\right), t \cdot \sigma(p)\right]=t \cdot \Theta(m, p)
\end{gathered}
$$

and hence it provides an isomorphism $N \cong M^{\left(e^{\prime}\right)} \times P$ as claimed.

Remark 7.2.6. Let us compute explicitly the projection $N \longrightarrow M$ arising from Proposition 7.2 .2 . At the level of $\mathbb{Z}$-modules,

$$
\mathrm{G}(N ; \sigma(e)) \longrightarrow \mathrm{G}\left(M ; e^{\prime}\right), \quad n \longmapsto \phi^{-1}(n-\sigma \psi(n))
$$

By recalling that the module structure is the one induced by the heap structure, we conclude that

$$
n-\sigma \psi(n)=\left[n, \sigma(e), \sigma \psi(n)^{-1}\right]=[n, \sigma(e),[\sigma(e), \sigma \psi(n), \sigma(e)]]=[n, \sigma \psi(n), \sigma(e)]
$$

Therefore, the projection $N \longrightarrow M$ is given by $n \longmapsto m_{n}$, where $m_{n} \in M$ is the unique element such that $\phi\left(m_{n}\right)=[n, \sigma \psi(n), \sigma(e)]$. Notice that this is not necessarily $T$-linear if $e$ or $\sigma(e)$ are not absorbers. The inverse to $\Theta$ is given by $\Theta^{-1}(n)=\left(m_{n}, \psi(n)\right)$.

At this point a curious reader may wonder why we introduced the terminology "split exact sequence" to refer to (7.2.2 and we did not use a more specific one instead, in order to distinguish (7.2.2 from (7.2.3) (such as e.g. "left" and "right" split exact 
sequences). The reason is that if $\psi: N \longrightarrow P$ admits a section $\sigma: P \longrightarrow N$, then $\sigma$ itself admits $\psi$ as a retraction. By applying Proposition 7.2 .2 to the split exact sequence

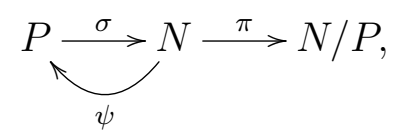

where $N / P$ is the quotient $T$-module with respect to the submodule $\sigma(P) \subseteq N$ and $\pi$ is the canonical projection, we conclude that $N \cong P \times N / P$ as $T$-modules. Now, $\pi \circ \phi$ is an isomorphism of Abelian heaps, since $\operatorname{Im}(\phi) \cap \operatorname{ker}_{\pi(\sigma(e))}(\pi)=\{\sigma(e)\}$ and for all $\pi(n) \in N / P,(\pi \circ \phi)\left(m_{n}\right)=\pi(n)$.

Let $T$ be a truss (not necessarily unital). Recall that epimorphisms in $T$-mod are surjective $T$-linear maps by Proposition 4.4.1.

Definition 7.2.7. Let $P$ be a $T$-module. We say that $P$ is projective if the functor $\operatorname{Hom}_{T}(P,-): T-\bmod \longrightarrow \mathbf{A h}$ preserves epimorphisms. That is to say, if for every surjective $T$-linear map $\pi: M \longrightarrow N$ and every $T$-linear map $f: P \longrightarrow N$ there exists a (not necessarily unique) T-linear map $\tilde{f}: P \longrightarrow M$ such that $\pi \circ \tilde{f}=f$. Diagrammatically,

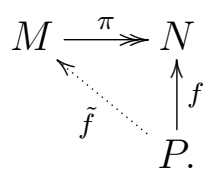

Proposition 7.2.8. A T-module $P$ satisfying the DBP property is projective. In particular, every tiny T-module is finitely generated and projective.

Proof. In view of Proposition 4.4.2, every epimorphism is a coequalizer. In particular, it is a colimit. By Theorem 7.1.11p $\operatorname{Hom}_{T}(P,-): T-\bmod \longrightarrow \mathbf{A h}$ is cocontinuous, and so it preserves small colimits and, in particular, epimorphisms. The last claim is a consequence of Theorem 7.1.111.

Remark 7.2.9. Proposition 7.2.8 should convince the reader that the terminology "smallprojective" from Definition 7.1 .12 would also be very well-suited for tiny objects in $T-\bmod$.

Lemma 7.2.10. Every projective T-module $P$ admits a T-linear morphism $f: P \longrightarrow T$. 
Proof. The required morphism is a filler of the following diagram

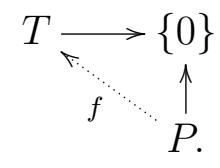

Proposition 7.2.11. Every free T-module is projective.

Proof. Let $X$ be any set, $\pi: M \longrightarrow N$ be a surjective $T$-linear map and $f: \mathcal{T}^{X} \longrightarrow N$ a $T$-linear map. Consider also the inclusion $\iota_{X}: X \longrightarrow \mathcal{T}^{X}, x \longmapsto 1 x$. For every $x \in X$ set $n_{x}:=f\left(\iota_{X}(x)\right) \in N$. Since $\pi$ is surjective, by the axiom of choice, for every $x \in X$, we may choose an $m_{x} \in M$ such that $\pi\left(m_{x}\right)=n_{x}$. This defines a function $\bar{f}: X \longrightarrow M, x \longmapsto m_{x}$. By the universal property of the free $T$-module, the latter extends uniquely to a $T$-linear map $\hat{f}: \mathcal{T}^{X} \longrightarrow M$ which satisfies $\pi(\hat{f}(1 x))=\pi\left(m_{x}\right)=$ $n_{x}=f(1 x)$. Since this implies that $f \circ \iota_{X}$ and $\pi \circ \hat{f} \circ \iota_{X}$ coincide, the uniqueness ensured by the universal property of the free $T$-modules entails that $f=\pi \circ \hat{f}$ as desired.

Corollary 7.2.12. Let $T$ be a truss without absorbers. Then any T-module with absorber cannot be projective. In particular, free T-modules over a truss without absorbers cannot have absorbers.

Proof. Since $T$-linear maps preserve absorbers, a projective $T$-module $P$ cannot have absorbers in view of Lemma 7.2.10. In particular, Proposition 7.2.11 entails that free modules over a truss without absorbers cannot have absorbers.

Remark 7.2.13. A truss $T$ has no absorbers if and only if there exists a non-empty $T$-module without absorbers. In fact, if $T$ admits an absorber $e$ then for every nonempty $T$-module $M$ and $m \in M, e \cdot m$ is an absorber in $M$. Conversely, $T$ itself is a $T$-module without absorbers. More precisely, a truss $T$ admits an absorber if and only if there exists a projective $T$-module admitting an absorber. Therefore, the hypothesis of Corollary 7.2.12 is not particularly restrictive.

Let us recall from Section 6.2 that functor $(-)_{\mathrm{Abs}}: \mathrm{T}(R)$-mod $\rightarrow R$-mod is given by $M \mapsto M / \operatorname{Abs}(M)$ and $\left(\varphi: M \rightarrow M^{\prime}\right) \mapsto\left(\varphi_{\text {Abs }}: \bar{m} \mapsto \overline{\varphi(m)}\right)$, see Lemma 6.2.6. 
Proposition 7.2.14. Let $R$ be a ring and $\mathrm{T}(R)$ be the associated truss.

1. If $P$ is projective over $\mathrm{T}(R)$ then $P_{\mathrm{Abs}}$ is projective over $R$.

2. If $P$ is finitely generated over $\mathrm{T}(R)$ then $P_{\mathrm{Abs}}$ is finitely generated over $R$.

In particular,

1. If $P$ is a tiny $\mathrm{T}(R)$-module then $P_{\mathrm{Abs}}$ is a finitely generated and projective $R$ module.

2. $P$ is a finitely generated and projective $R$-module if and only if $\mathrm{T}(P)$ is a tiny $\mathrm{T}(R)$-module.

Proof. To prove 1, let $\pi: M \longrightarrow N$ be a surjective morphism of $R$-modules and assume that $f: P_{\mathrm{Abs}} \longrightarrow N$ is an $R$-linear map. Since the action of the functor $\mathrm{T}$ on morphisms does not change the underlying mapping and since epimorphisms in $\mathrm{T}(R)$-mod and $R$-mod are exactly surjective maps (see Proposition 4.4.1), the functor $\mathrm{T}$ preserves epimorphisms, and hence we can consider the diagram of $\mathrm{T}(R)$-modules

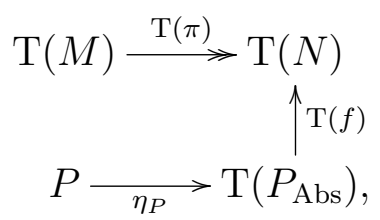

where $\eta_{P}: P \longrightarrow \mathrm{T}\left(P_{\mathrm{Abs}}\right)$ is the unit of the adjunction $(-)_{\mathrm{Abs}} \dashv \mathrm{T}$. Since $P$ is projective over $\mathrm{T}(R)$, there exists a filler $f^{\prime}$ rendering the following diagram commutative:

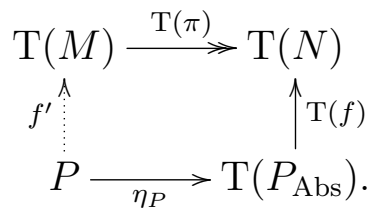

By applying the functor $(-)_{\text {Abs }}$ to the latter diagram, we find the commutative diagram

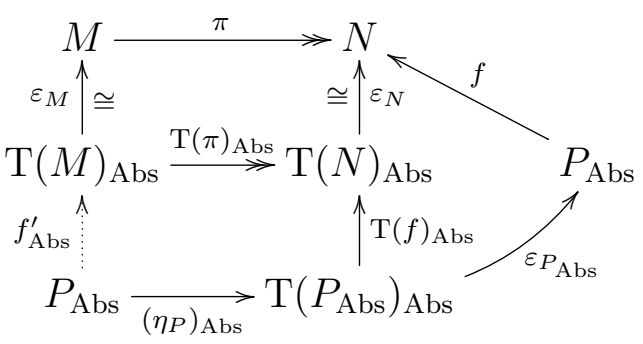


and since $\varepsilon_{P_{\mathrm{Abs}}} \circ\left(\eta_{P}\right)_{\mathrm{Abs}}=1_{P}$, we constructed a morphism of $R$-modules $\hat{f}:=\varepsilon_{M} \circ f_{\mathrm{Abs}}^{\prime}$ : $P_{\text {Abs }} \longrightarrow M$ such that $\pi \circ \hat{f}=f$.

To prove 2, pick an epimorphism $\pi: \stackrel{n}{\boxplus} \mathrm{T}(R) \longrightarrow P$. Since $(-)_{\mathrm{Abs}}$ is a left adjoint functor (see Lemma 6.2.6) and every epimorphism in $\mathrm{T}(R)$-mod is a coequalizer (see Proposition 4.4.2,$(-)_{\text {Abs }}$ preserves epimorphisms and coproducts, and hence

$$
R^{n} \cong \bigoplus^{n} \mathrm{~T}(R)_{\mathrm{Abs}} \cong(\stackrel{n}{\boxplus} \mathrm{T}(R))_{\mathrm{Abs}} \stackrel{\pi_{\mathrm{Abs}}}{\longrightarrow} P_{\mathrm{Abs}}
$$

is an epimorphism of $R$-modules, showing that $P_{\mathrm{Abs}}$ is finitely generated.

Concerning the last claims, assume that $P$ is tiny over $\mathrm{T}(R)$. Then it is finitely generated and projective by Proposition 7.2.8, and hence $P_{\mathrm{Abs}}$ is finitely generated and projective over $R$, proving 1. Furthermore, in view of Example 7.1.14 we know that if $P$ is finitely generated and projective over $R$, then $\mathrm{T}(P)$ is tiny over $\mathrm{T}(R)$. Conversely, we have just seen that if $\mathrm{T}(P)$ is tiny over $\mathrm{T}(R)$, then $\mathrm{T}(P)_{\mathrm{Abs}} \cong P$ is finitely generated and projective over $R$, thus showing 2

Lemma 7.2.15. The empty T-module is projective.

Proof. For every $T$-module $M$, there exists a unique morphism $\varnothing \longrightarrow M$ which is the empty morphism. Therefore, the following diagram is commutative and gives a lifting of the empty morphism along the epimorphism $\pi$ :

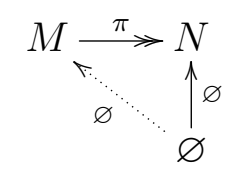

Proposition 7.2.16. Let $M$ be a non-empty projective T-module. Then $M$ is a direct factor of a free T-module. More precisely, there exist a set $X$ and a T-module with absorber $P$ such that $M \times P \cong \mathcal{T}^{X}$ as $T$-modules.

Proof. Since every $T$-module is a quotient of a free one (as we showed at the end of Section 6.3), there exists a set $X$ and a surjective $T$-linear morphism $\gamma: \mathcal{T}^{X} \longrightarrow M$. By projectivity of $M, \gamma$ admits a $T$-linear section $\phi: M \longrightarrow \mathcal{T}^{X}$. Thus, $\phi$ is injective and we may identify $M$ with the $T$-submodule $\phi(M) \subseteq \mathcal{T}^{X}$. Consider now

$$
P:=\mathcal{T}^{X} / \sim_{\phi(M)} \cong \mathcal{T}^{X} / M,
$$


which is a $T$-module with absorber. Denote by $\psi: \mathcal{T}^{X} \longrightarrow P$ the quotient map. As $M$ is non-empty, the sequence

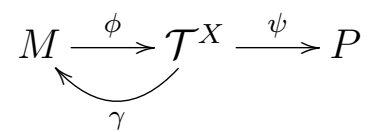

is split exact with $\psi$ surjective and so, by Proposition $7.2 .2, \mathcal{T}^{X} \cong M \times P$ as $T$ modules.

The converse of Proposition 7.2.16 holds as well.

Proposition 7.2.17. Let $M$ be a T-module. If there exists a $T$-module $P$ with absorber and a set $X$ such that $\mathcal{T}^{X} \cong M \times P$, then $M$ is projective.

Proof. Let $e \in P$ be an absorber. Then the assignment $\phi: M \longrightarrow M \times P, m \longmapsto(m, e)$, is a well-defined injective $T$-linear morphism, providing a section for the canonical projection $\gamma: M \times P \longrightarrow M,(m, p) \longmapsto m$. As a consequence, for every surjective morphism $g: N \longrightarrow Q$ of $T$-modules and every $T$-linear map $f: M \longrightarrow Q$, we can consider the diagram of $T$-linear maps

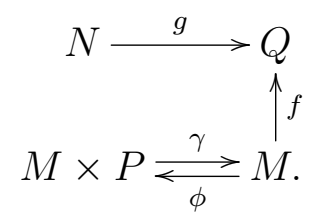

By projectivity of $\mathcal{T}^{X}$, there exists $\tilde{f}: \mathcal{T}^{X} \longrightarrow N$ such that the diagram

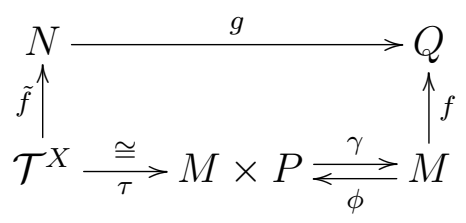

commutes, that is, $g \circ \tilde{f}=f \circ \gamma \circ \tau$. If we set $\hat{f}:=\tilde{f} \circ \tau^{-1} \circ \phi$ then

$$
g \circ \hat{f}=g \circ \tilde{f} \circ \tau^{-1} \circ \phi=f \circ \gamma \circ \phi=f
$$

whence $f: M \longrightarrow Q$ can be lifted to a $T$-linear map $\hat{f}: M \longrightarrow N$ along $g$, that is, $g \circ \hat{f}=f$, proving that $M$ is projective. 
Theorem 7.2.18. A T-module $M$ is projective if and only if there exists a T-module with absorber $P$ such that $M \times P$ is a free $T$-module.

Proof. It follows from Propositions 7.2.16 and 7.2.17.

Proposition 7.2.19. Let $P$ be a tiny $T$-module with dual basis $\left\{\left(e_{1}, \ldots, e_{s}\right),\left(\phi_{1}, \ldots, \phi_{s}\right)\right\}$. Then there exists a T-module $Q$ with an absorber, such that $P \times Q \cong T^{s}$.

Proof. By the universal property of the direct product, there exists a unique morphism of $T$-modules $\phi: P \longrightarrow T^{s}$ such that $\pi_{k} \circ \phi=\phi_{k}$, where $\pi_{k}: T^{s} \longrightarrow T$ is the projection on the $k$-th factor. The other way around, consider the assignment

$$
\pi: T^{s} \longrightarrow P, \quad\left(t_{1}, \ldots, t_{s}\right) \longmapsto\left[t_{1} \cdot e_{1}, \ldots, t_{s} \cdot e_{s}\right]
$$

Since

$$
\begin{aligned}
\pi\left(\left[\left(t_{1}, \ldots, t_{s}\right),\right.\right. & \left.\left.\left(t_{1}^{\prime}, \ldots, t_{s}^{\prime}\right),\left(t_{1}^{\prime \prime}, \ldots, t_{s}^{\prime \prime}\right)\right]\right)=\pi\left(\left(\left[t_{1}, t_{1}^{\prime}, t_{1}^{\prime \prime}\right], \ldots,\left[t_{s}, t_{s}^{\prime}, t_{s}^{\prime \prime}\right]\right)\right) \\
= & {\left[\left[t_{1}, t_{1}^{\prime}, t_{1}^{\prime \prime}\right] \cdot e_{1}, \ldots,\left[t_{s}, t_{s}^{\prime}, t_{s}^{\prime \prime}\right] \cdot e_{s}\right] } \\
= & {\left[\left[t_{1} \cdot e_{1}, t_{1}^{\prime} \cdot e_{1}, t_{1}^{\prime \prime} \cdot e_{1}\right], \ldots,\left[t_{s} \cdot e_{s}, t_{s}^{\prime} \cdot e_{s}, t_{s}^{\prime \prime} \cdot e_{s}\right]\right] } \\
& \stackrel{[1.1 .2]}{=}\left[\left[t_{1} \cdot e_{1}, \ldots, t_{s} \cdot e_{s}\right],\left[t_{1}^{\prime} \cdot e_{1}, \ldots, t_{s}^{\prime} \cdot e_{s}\right],\left[t_{1}^{\prime \prime} \cdot e_{1}, \ldots, t_{s}^{\prime \prime} \cdot e_{s}\right]\right] \\
= & {\left[\pi\left(t_{1}, \ldots, t_{s}\right), \pi\left(t_{1}^{\prime}, \ldots, t_{s}^{\prime}\right), \pi\left(t_{1}^{\prime \prime}, \ldots, t_{s}^{\prime \prime}\right)\right] }
\end{aligned}
$$

for all $\left(t_{1}, \ldots, t_{s}\right),\left(t_{1}^{\prime}, \ldots, t_{s}^{\prime}\right),\left(t_{1}^{\prime \prime}, \ldots, t_{s}^{\prime \prime}\right) \in T^{s}$ and

$$
\begin{aligned}
\pi\left(t \cdot\left(t_{1}, \ldots, t_{s}\right)\right) & =\pi\left(\left(t t_{1}, \ldots, t t_{s}\right)\right)=\left[t t_{1} \cdot e_{1}, \ldots, t t_{s} \cdot e_{s}\right] \\
& =t \cdot\left[t_{1} \cdot e_{1}, \ldots, t_{s} \cdot e_{s}\right]=t \cdot \pi\left(\left(t_{1}, \ldots, t_{s}\right)\right),
\end{aligned}
$$

for all $t \in T, \pi$ is a morphism of left $T$-modules satisfying $\pi \circ \phi=1_{P}$ (because $P$ satisfies the DBP). Thus, $\phi$ is injective and we may identify $P$ with the submodule $\phi(P) \subseteq T^{s}$. As in the proof of Proposition 7.2.16, consider $Q:=T^{s} / \sim_{\phi(P)} \cong T^{s} / P$, which is a $T$-module with absorber, and the quotient map $\psi: T^{s} \longrightarrow Q$. By 1 of Example 7.1.9, $P$ is non-empty, and so the sequence

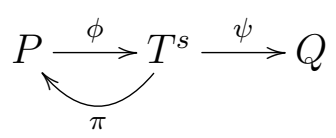

is split exact with $\psi$ surjective. By Proposition 7.2.2, $T^{s} \cong P \times Q$ as $T$-modules. 
Differently from what we have seen for projective modules, it seems that the converse of Proposition 7.2 .19 requires stronger hypotheses.

Proposition 7.2.20. Let $T$ be a unital truss with left absorber $a \in T$. If there exist an odd positive integer $s=2 k+1$ and $T$-modules $P, Q$ such that $T^{s} \cong P \times Q$ as left T-modules, then both $P$ and $Q$ are tiny $T$-modules.

Proof. If $T$ admits a left absorber $a$ and $\gamma: T^{s} \longrightarrow P \times Q$ is an isomorphism as left $T$ modules, then $(a, a, \ldots, a) \in T^{s}$ is a left absorber and so $a_{P}:=\pi_{P}(\gamma((a, a, \ldots, a))) \in P$ and $a_{Q}:=\pi_{Q}(\gamma((a, a, \ldots, a))) \in Q$ are absorbers as well.

Now, set $1_{i}:=\left(a, \ldots, a, 1_{T}, a, \ldots, a\right)$ where $1_{T}$ appears in the $i$-th position. Consider the projection $\pi:=\left(T^{s} \stackrel{\gamma}{\longrightarrow} P \times Q \stackrel{\pi_{P}}{\longrightarrow} P\right)$, the elements $e_{k}:=\pi\left(1_{k}\right) \in P$ and the compositions $\phi_{k}:=\left(P \longrightarrow P \times Q \stackrel{\gamma^{-1}}{\longrightarrow} T^{s} \stackrel{\pi_{k}}{\longrightarrow} T\right), p \longmapsto \pi_{k} \gamma\left(p, a_{Q}\right)$, for all $k=1, \ldots, s$. For all $p \in P$, one finds that

$$
\left[\phi_{1}(p) \cdot e_{1}, \ldots, \phi_{s}(p) \cdot e_{s}\right]=\pi\left(\left[\phi_{1}(p) \cdot 1_{1}, \ldots, \phi_{s}(p) \cdot 1_{s}\right]\right)=\pi\left(\left(\phi_{1}(p), \ldots, \phi_{s}(p)\right)\right)=p
$$

and so $P$ satisfies the DBP. The proof for $Q$ is analogous. 


\section{Appendix A}

\section{Categories}

This appendix aims to familiarise or remind the reader of the basics of category theory. We recall definitions of categories, functors, adjoints, limits and colimits. It is based on [24], [39] and [40]. Another book worth recommendation is [17]. Let us start with a definition of a category;

Definition A.0.1. A category $\mathfrak{C}$ consists of

- a collection of objects, denoted by $o b(\mathfrak{C})$;

- for each $A, B \in o b(\mathfrak{C})$, a collection, denoted by $\mathfrak{C}(A, B)$ or $\operatorname{Hom}(A, B)$, of maps or arrows or morphisms from $A$ to $B$;

- for each $A, B, C \in o b(\mathfrak{C})$, a function

$$
\begin{gathered}
\mathfrak{C}(B, C) \times \mathfrak{C}(A, B) \rightarrow \mathfrak{C}(A, C) \\
(g, f) \mapsto g \circ f
\end{gathered}
$$

called composition;

- for each $A \in o b(\mathfrak{C})$, an element $1_{A}$ of $\mathfrak{C}(A, A)$, called the identity on $\mathrm{A}$,

satisfying the following axioms:

- associativity: for each $f \in \mathfrak{C}(A, B), g \in \mathfrak{C}(B, C)$ and $h \in \mathfrak{C}(C, D)$, we have $(h \circ g) \circ f=h \circ(g \circ f)$; 
- identity laws: for each $f \in \mathfrak{C}(A, B), f \circ 1_{A}=f=1_{B} \circ f$.

Example A.0.2. Most common examples of categories are:

- category consisted of sets as objects and functions as morphisms, denoted by Set;

- category consisted of groups as objects and group homomorphisms as morphisms, denoted by Grp;

- category consisted of Abelian groups as objects and group homomorphisms as morphisms, denoted by Ab.

Observe that a collection is not defined. For our purpose, one can consider only classes, so there exists a class of all sets. A class of all classes does not exist. For the bigger picture, through Universes, I recommend reading Chapter 6 of [17]. In the category theory, whenever one works with sets, one works with small objects. Therefore, whenever the word small appears, and it refers to a category theory, it means that one works with "something at most as big as" sets. For example, a locally small category means that collections of morphisms are sets.

Definition A.0.3. An opposite or dual category $\mathfrak{C}^{o p}$ of a category $\mathfrak{C}$ consists of $o b\left(\mathfrak{C}^{o p}\right)=$ $o b(\mathfrak{C})$ and $\mathfrak{C}^{o p}(A, B)=\mathfrak{C}(B, A)$.

Definition A.0.4. A subcategory $\mathfrak{K}$ of a category $\mathfrak{C}$ consists of sub-collections of $o b(\mathfrak{C})$ and $\mathfrak{C}(A, B)$, for all $A, B \in o b(\mathfrak{K})$, and satisfy axioms of a category. If for all $A, B \in o b(\mathfrak{K}), \mathfrak{C}(A, B)=\mathfrak{K}(A, B)$, then $\mathfrak{K}$ is called a full subcategory of a category $\mathfrak{C}$.

Example A.0.5. The category Ab is a full subcategory of the category Grp.

Definition A.0.6. Let $\mathfrak{C}$ be a category and $A, B \in o b(\mathfrak{C})$. Then a morphism $f \in$ $\mathfrak{C}(A, B)$ is

(1) a monomorphism if for all $C \in o b(\mathfrak{C})$ and $g, g^{\prime} \in \mathfrak{C}(C, A), f \circ g=f \circ g^{\prime}$ implies $g=g^{\prime}$

(2) an epimorphism if for all $C \in o b(\mathfrak{C})$ and $g, g^{\prime} \in \mathfrak{C}(A, C), g \circ f=g^{\prime} \circ f$ implies $g=g^{\prime}$ 
(3) an isomorphism if there exists $g \in \mathfrak{C}(B, A)$ such that $g \circ f=1_{A}$ and $f \circ g=1_{B}$.

Definition A.0.7. Let $\mathfrak{C}$ and $\mathfrak{D}$ be categories. A functor $\mathrm{F}: \mathfrak{C} \rightarrow \mathfrak{D}$ consists of

- a function

$$
o b(\mathfrak{C}) \rightarrow o b(\mathfrak{D})
$$

written as $C \mapsto \mathrm{F}(C)$;

- for each $C, C^{\prime} \in o b(\mathfrak{C})$, a function

$$
\mathfrak{C}\left(C, C^{\prime}\right) \rightarrow \mathfrak{D}\left(\mathrm{F}(C), \mathrm{F}\left(C^{\prime}\right)\right)
$$

satisfying the following axioms:

- $\mathrm{F}\left(f^{\prime} \circ f\right)=\mathrm{F}\left(f^{\prime}\right) \circ \mathrm{F}(f)$ for all $f \in \mathfrak{C}(A, B)$ and $f^{\prime} \in \mathfrak{C}(B, C)$;

- $\mathrm{F}\left(1_{A}\right)=1_{\mathrm{F}(A)}$ for all $A \in o b(\mathfrak{C})$.

Example A.0.8. Let us consider two categories Grp and Set. Then the assignment $\mathrm{U}:$ Grp $\rightarrow$ Set given by taking a group and forgetting the group structure on it, i.e. taking an underlying set, is a functor that maps every group homomorphism into an underlying function. This kind of functor that forgets the structure of objects is called a forgetful functor. Analogously, one can assign to every set a group by taking a free group over the set. Then every function between sets extends to a homomorphism between free groups, and F : Set $\rightarrow$ Grp is the functor given by free construction. A functor given by a free construction is called a free functor.

Definition A.0.9. Let $\mathfrak{C}, \mathfrak{D}$ be categories and let $F: \mathfrak{C} \rightarrow \mathfrak{D}, G: \mathfrak{C} \rightarrow \mathfrak{D}$ be functors. A natural transformation $\alpha: \mathrm{F} \rightarrow \mathrm{G}$ is a family of morphisms in $\mathfrak{D}, \alpha_{A}: \mathrm{F}(A) \rightarrow \mathrm{G}(A)$ iterated by $A \in \mathfrak{C}$ such that for every morphisms $f: A \rightarrow A^{\prime}$, the square diagram:

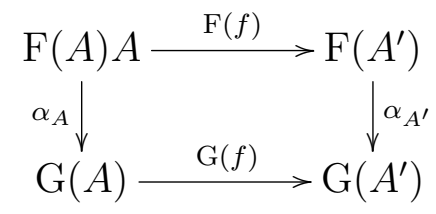

commutes. The maps $\alpha_{A}$ are called the components of $\alpha$. 
Definition A.0.10. A functor $\mathrm{F}: \mathfrak{C} \rightarrow \mathfrak{D}$ is faithful (respectively, full) if for all $A, B \in$ $o b(\mathfrak{C})$, the function: $\mathfrak{C}(A, B) \rightarrow \mathfrak{D}(\mathrm{F}(A), \mathrm{F}(B)), f \mapsto F(f)$ is injective (respectively, surjective).

Definition A.0.11. A functor $\mathrm{F}: \mathfrak{C} \rightarrow \mathfrak{D}$ is essentially surjective on objects if for all $B \in \mathfrak{D}$, there exists $A \in \mathfrak{C}$ such that $\mathrm{F}(A) \cong B$.

Definition A.0.12. We say that two functors $\mathrm{F}, \mathrm{G}: \mathfrak{C} \rightarrow \mathfrak{D}$ are naturally isomorphic if there exists a natural transformation between them such that all components are isomorphisms in $\mathfrak{D}$. A natural isomorphism is a natural transformation for which all components are isomorphisms.

Definition A.0.13. Given two functors F, G : $\mathfrak{C} \rightarrow \mathfrak{D}$, we say that

$$
\mathrm{F}(A) \cong \mathrm{G}(A) \text { naturally in } A,
$$

if $\mathrm{F}$ and $\mathrm{G}$ are naturally isomorphic.

Definition A.0.14. An equivalence between categories $\mathfrak{C}$ and $\mathfrak{D}$ consists of a pair of functors, $\mathrm{F}: \mathfrak{C} \rightarrow \mathfrak{D}$ and $\mathrm{G}: \mathfrak{D} \rightarrow \mathfrak{C}$, together with natural isomorphisms

$$
\eta: 1_{\mathfrak{C}} \rightarrow \mathrm{G} \circ \mathrm{F}, \quad \varepsilon: \mathrm{F} \circ \mathrm{G} \rightarrow 1_{\mathfrak{D}}
$$

If there exists an equivalence between $\mathfrak{C}$ and $\mathfrak{D}$, we say that $\mathfrak{C}$ and $\mathfrak{D}$ are equivalent.

Proposition A.0.15. A functor $\mathrm{F}: \mathfrak{C} \rightarrow \mathfrak{D}$ is an equivalence if and only if it is full, faithful and essentially surjective on objects.

Definition A.0.16. Let $\mathfrak{C}$ and $\mathfrak{D}$ be categories. An adjunction from $\mathfrak{C}$ to $\mathfrak{D}$ is a triple $(\mathrm{F}, \mathrm{G}, \varphi): \mathfrak{C} \rightarrow \mathfrak{D}$, where $\mathrm{F}: \mathfrak{C} \rightarrow \mathfrak{D}$ and $\mathrm{G}: \mathfrak{D} \rightarrow \mathfrak{C}$ are functors, while $\varphi$ is a function which assingns to each pair of objects $C \in o b(\mathfrak{C})$ and $D \in o b(\mathfrak{D})$ a bijection

$$
\varphi=\varphi_{C, D}: \mathfrak{D}(\mathrm{F}(C), D) \rightarrow \mathfrak{C}(C, \mathrm{G}(D))
$$

which is natural in $C$ and $D$, where naturality in $C$ and $D$ means that $\varphi$ assigns to each arrow $f: \mathrm{F}(C) \rightarrow D$, an arrow $\varphi(f): C \rightarrow \mathrm{G}(D)$, the right adjunct of $f$, in such a way that following naturality conditions holds:

$$
\varphi(f \circ \mathrm{F}(h))=\varphi(f) \circ h, \quad \varphi(k \circ f)=\mathrm{G}(K) \circ \varphi(f),
$$


for all $f$ and all arrows $h: C^{\prime} \rightarrow C$ and $k: D \rightarrow D^{\prime}$. Given such adjunction, the functor $F$ is said to be a left adjoint to $G$, while $G$ is called a right adjoint to $F$. We denote by $F \dashv G$ that $F$ is a left adjoint to $G$.

Example A.0.17. The free functor is a left adjoint to the forgetful functor.

Theorem A.0.18. (see [24], Theorem 1 and Theorem 2, pages 80-81) Each adjunction $(\mathrm{F}, \mathrm{G}, \varphi): \mathfrak{C} \rightarrow \mathfrak{D}$ is completely determined by functors $\mathrm{F}: \mathfrak{C} \rightarrow \mathfrak{D}, \mathrm{G}: \mathfrak{D} \rightarrow \mathfrak{C}$ and natural transformations $\eta: 1_{\mathfrak{C}} \rightarrow G F$, the unit, and $\varepsilon: F G \rightarrow 1_{\mathfrak{D}}$, the counit, such that composities

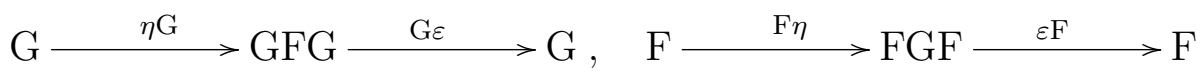

are the identity transformations. In this case bijection $\varphi$ is given by $\varphi(f)=\mathrm{G}(f) \circ \eta_{C}$ for all $f \in \mathfrak{D}(\mathrm{F}(C), D)$, similarly $\varphi^{-1}(g)=\varepsilon_{D} \circ \mathrm{F}(g)$ for all $g \in \mathfrak{C}(C, \mathrm{G}(C))$.

Definition A.0.19. Let $\mathfrak{C}$ be a category and $\mathfrak{I}$ be a small category, i.e. a category such that collections of objects and morphisms are sets. A functor D : $\mathfrak{I} \rightarrow \mathfrak{C}$ is called a diagram in $\mathfrak{C}$ of shape $\mathfrak{I}$.

Definition A.0.20. Let $\mathrm{D}: \mathfrak{I} \rightarrow \mathfrak{C}$ be a diagram in $\mathfrak{C}$.

(1) A cone on $\mathrm{D}$ is an object $C \in \operatorname{ob}(\mathfrak{C})$ together with a family $\left\{f_{I}: A \rightarrow D(I) \mid I \in\right.$ $o b(\mathfrak{I})\}$ of maps in $\mathfrak{C}$ such that for all maps $u: I \rightarrow J$ in $\mathfrak{I}$, the triangle

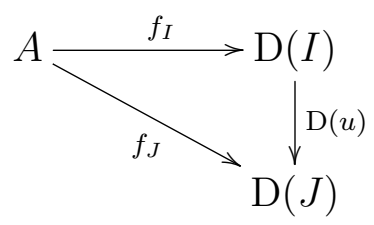

commutes

(2) A limit of $\mathrm{D}$ is a cone $\left\{p_{I}: L \rightarrow \mathrm{D}(I) \mid I \in o b(\mathfrak{I})\right\}$ with the property that for any cone $\left\{f_{I}: A \rightarrow \mathrm{D}(I) \mid I \in o b(\mathfrak{I})\right\}$ on $\mathrm{D}$, there exists a unique map $\bar{f}: A \rightarrow L$ such that $p_{I} \circ \bar{f}=f_{I}$ for all $I \in o b(\mathfrak{I})$. The maps $p_{I}$ are called the projections of the limit. If $\mathfrak{I}$ is a small category then a limit of D is called a small limit of $\mathrm{D}$. 
Example A.0.21. An object $I$ of a category $\mathfrak{C}$ is called an initial object if for all objects $C \in \operatorname{ob}(\mathfrak{C})$ there exists only one arrow $f: I \rightarrow C$. An initial object property is a limit.

Example A.0.22. Being a monomorphism is a limit.

Example A.0.23. A product of $A, B \in \mathfrak{C}$ is a triple $\left(P, p_{A}, p_{B}\right)$, where $P \in o b(\mathfrak{C})$, $p_{A}: P \rightarrow A$ and $p_{B}: P \rightarrow B$ such that for any triple $\left(L, f_{A}, f_{B}\right)$, where $L \in o b(\mathfrak{C})$, $f_{A}: L \rightarrow A$ and $f_{B}: L \rightarrow B$, exists a unique morphism $\bar{f}: L \rightarrow P$ such that $f_{A}=p_{A} \circ \bar{f}$ and $f_{B}=p_{B} \circ \bar{f}$. A product property is a limit.

Example A.0.24. Let $\mathfrak{C}$ be a category and $s, t: X \rightarrow Y$ be morphisms in this category. An equalizer of $s$ and $t$ is a pair $(E, i)$, where $E \in o b(\mathfrak{C})$ and $i \in \mathfrak{C}(E, X)$ such that $s \circ i=t \circ i$ and for any other pair $(S, j)$ with the same property $s \circ j=t \circ j$, there exists exactly one morphism $f: S \rightarrow E$ such that $i \circ f=j$. In groups a kernel is an equalizer. An equalizer property is a limit.

Example A.0.25. A pullback is a limit of the following diagram

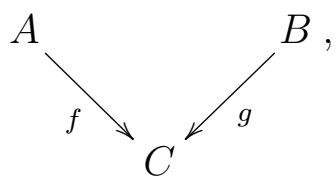

in $\mathfrak{C}$. Thus, a pullback of the preceding diagram in $\mathfrak{C}$ is a cone $\left(A \times_{C} B, p_{A}, p_{B}\right)$, where $A \times_{C} B$ is an object in $\mathfrak{C}, p_{A}: A \rightarrow C$ and $p_{B}: B \rightarrow C$ are morphisms such that $f \circ p_{A}=g \circ p_{B}$

Definition A.0.26. Let $\mathfrak{C}$ be a category and $\mathfrak{I}$ be a small category. Let $D: \mathfrak{I} \rightarrow \mathfrak{C}$ be a diagram in $\mathfrak{C}$ and consider the coresponding functor $D^{o p}: \mathfrak{I}^{o p} \rightarrow \mathfrak{C}^{o p}$. A cocone on $D$ is a cone on $D^{o p}$. A colimit $D$ is a limit of $D^{o p}$. If $\mathfrak{I}$ is a small category then a colimit of $D$ is called a small colimit of $D$.

Example A.0.27. An object $I$ of a category $\mathfrak{C}$ is called a terminal object if for all objects $C \in o b(\mathfrak{C})$ there exists only one arrow $f: C \rightarrow I$. A terminal object property is a colimit.

Example A.0.28. Being an epimorphism is a colimit. 
Example A.0.29. Let $\mathfrak{C}$ be a category and $s, t: X \rightarrow Y$ be morphisms in this category. A coequalizer of $s$ and $t$ is a pair $(E, i)$, where $E \in o b(\mathfrak{C})$ and $i \in \mathfrak{C}(Y, E)$ such that $i \circ s=i \circ t$ and for any other pair $(S, j)$ with the same property $j \circ s=j \circ t$, there exists exactly one morphism $f: E \rightarrow S$ such that $f \circ i=j$. A coequalizer property is a colimit.

Example A.0.30. A coproduct of $A, B \in \mathfrak{C}$ is a triple $\left(C, i_{A}, i_{B}\right)$, where $C \in$ ob( $(\mathfrak{C})$, $i_{A}: \rightarrow P$ and $i_{B}: B \rightarrow P$ such that for any triple $\left(L, f_{A}, f_{B}\right)$, where $L \in o b(\mathfrak{C})$, $f_{A}: A \rightarrow L$ and $f_{B}: B \rightarrow L$, exists a unique morphism $\bar{f}: P \rightarrow L$ such that $f_{A}=i_{A} \circ \bar{f}$ and $f_{B}=i_{B} \circ \bar{f}$. A coproduct property is a colimit.

Example A.0.31. A pushout is a colimit of the following diagram

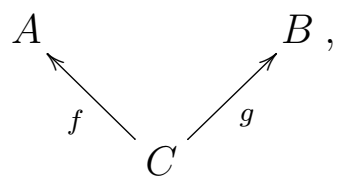

in $\mathfrak{C}$. Thus, a pushout of the preceding diagram in $\mathfrak{C}$ is a cocone $\left(A+_{C} B, i_{A}, i_{B}\right)$, where $A+{ }_{C} B$ is an object in $\mathfrak{C}, i_{A}: A \rightarrow C$ and $i_{B}: B \rightarrow C$ are morphisms such that $i_{A} \circ f=i_{B} \circ g$.

Theorem A.0.32. (see [24], Theorem 1, pages 114-115). A functor that has a left adjoint preserves limits, analogously a functor that has a right adjoint preserves colimits.

Definition A.0.33. A complete category is a category in which all small limits exist. Analogously, a cocomplete category is a category in which all small colimits exist.

Definition A.0.34. A functor is called continuous if it preserves all small limits. Analogously, a functor is called cocontinuous if it preserves all small colimits.

Theorem A.0.35 ([24, Theorem IV.3.1]). For an adjunction $(\mathrm{F}, \mathrm{G}, \epsilon, \eta): \mathfrak{C} \rightarrow \mathfrak{B}:$

1. A functor $\mathrm{G}: \mathfrak{B} \rightarrow \mathfrak{C}$ is faithful if and only if every morphism of counit $\epsilon$ is an epimorphism. 
2. A functor $\mathrm{G}$ is full if and only if every morphism of counit $\epsilon$ is a split monic, i.e. every component of counit is a monomorphism such that there exists a retraction to this monomorphism.

Therefore $\mathrm{G}$ is fully faithful if and only if every component of counit $\epsilon_{X}: \mathrm{FG}(X) \rightarrow X$ is an isomorphism. 


\section{Appendix B}

\section{Structures with binary operations}

In this appendix, we introduce basics related to sets with binary operations. The first section focuses on a set with one binary operation, i.e. semigroup, monoid and group. The second section consists of facts related to a set with two binary operations. For example, near-rings and skew braces.

\section{B.1 Semigroups, monoids and groups}

\section{Definition B.1.1.}

(1) A semigroup is a pair of a set $S$ and an associative binary operation $\cdot: S \times S \rightarrow S$.

(2) A monoid is a semigroup $S$ with an identity element, i.e. there exists an identity $1 \in S$ such that for all $s \in S, 1 \cdot s=s \cdot 1=s$

(3) A group is a monoid in which every element has an inverse element, i.e. for all $s \in S$ exists an inverse $s^{-1} \in S$ such that $s \cdot s^{-1}=s^{-1} \cdot s=1$.

Definition B.1.2. A map $f: S \rightarrow S^{\prime}$ between semigroups (groups) is called a semigroup (group) homomorphism if for all $s, h \in S$,

$$
f(s \cdot h)=f(s) \cdot f(h) .
$$

Moreover if $f$ is a semigroup homomorphism between monoids such that $f$ maps an identity to an identity is called a homomorphism of monoids. 
Definition B.1.3. Let $M$ be a monoid. A multiplicative closed subset $S \subseteq M$ containing a unit element in a monoid $M$ is a left (right) Ore set if it satisfies the

- (left (right) cancellability) If for all $n, m \in M$ and $s \in S, n s=m s(s n=s m)$, then there exists $s^{\prime} \in S$ such that $s^{\prime} n=s^{\prime} m\left(n s^{\prime}=m s^{\prime}\right)$.

- (left (right) Ore condition) For any $r \in R$ and $s \in S$ there exists $r^{\prime} \in R$ and $s^{\prime} \in S$ such that $s^{\prime} r=r^{\prime} s\left(r s^{\prime}=s r^{\prime}\right)$. A subset $S$ of a monoid is called an Ore set if it is left and right Ore set.

Lemma B.1.4. A group homomorphism is an epimorphism (a monomorphism) if and only if it is surjective (injective).

Proof. Surjective and injective group homomorphisms are epimorphisms and monomorphisms. That follows as group homomorphism is a function. Every monomorphism is injective is relatively easy and follows a similar way to the case of heaps. See proof of Lemma 1.1.8. Every epimorphism is surjective can be found in [41].

Construction B.1.5. We will briefly discuss the construction of a free group. We will omit to check that relations are congruences as this construction is standard and well-known. Let $X$ be a set. We construct a free group over a set $X, \mathcal{G}(X)$ as follows. We take a disjoint union of three sets $X,\{e\}$ and $X^{-1}:=\left\{x^{-1} \mid x \in X\right\}$, where $\{e\}$ is a singleton set, $X^{-1}$ and $X$ are bijective sets. Consider a set

$$
W(X)=\left\{x_{1} \ldots x_{n} \mid n \in \mathbb{N}, x_{i} \in X \sqcup X^{-1} \sqcup\{e\}\right\}
$$

of all finite words constructed from letters of $X \sqcup X^{-1} \sqcup\{e\}$. Now $W(X)$ with a binary operation given by juxtaposition, i.e. glueing words

$$
x_{1} \ldots x_{n}+y_{1} \ldots y_{m}=x_{1} \ldots x_{n} y_{1} \ldots y_{m}
$$

is a semigroup, i.e. a free semigroup over a set $X \sqcup X^{-1} \sqcup\{e\}$. Now, let us generate a congruence $\sim$ by

$x_{1} \ldots x_{i} e x_{i+1} \ldots x_{n} \sim x_{1} \ldots x_{n}$, for all $n \in \mathbb{N}, i \in\{1, \ldots, n\}$ and $x_{i} \in X \sqcup X^{-1} \sqcup\{e\}$. 
Then $W(X) / \sim$ with juxtaposition is a monoid, i.e. a free monoid over a set $X \sqcup X^{-1}$. Last step is to construct inverses, to do so let us generate another congruence $\sim_{i n v}$ by

$$
x_{1} \ldots x_{i} x x^{-1} x_{i+1} \ldots x_{n} \sim_{i n v} x_{1} \ldots x_{i} x^{-1} x x_{i+1} \ldots x_{n} \sim_{i n v} x_{1} \ldots x_{i} e x_{i+1} \ldots x_{n}
$$

for all $n \in \mathbb{N}, i \in\{1, \ldots, n\}$ and $x, x^{-1}, x_{i} \in X \sqcup X^{-1} \sqcup\{e\}$.

Then $\mathcal{G}(X):=W(X) / \sim / \sim_{i n v}$ together with a juxtaposition is a free group with a neutral element $e$.

Corollary B.1.6. Since $\mathcal{G}(X)$ is defined for all sets and every function between sets uniquely extends to a homomorphism between free groups, an assignment $\mathcal{G}:$ Set $\rightarrow$ Grp is a functor. Moreover, it is a left adjoint to the forgetful functor $\mathrm{U}_{\mathbf{G r p}}$ : Grp $\rightarrow$ Set.

Definition B.1.7. A sequence of Abelian groups

$$
G_{0} \stackrel{f_{1}}{\longrightarrow} G_{1} \stackrel{f_{2}}{\longrightarrow} G_{2} \stackrel{f_{3}}{\longrightarrow} \ldots \stackrel{f_{n}}{\longrightarrow} G_{n}
$$

is exact if for all $i \in\{1, \ldots, n\}, \operatorname{Im}\left(f_{i}\right)=\operatorname{ker}\left(f_{i+1}\right)$. A short exact sequence is an exact sequence of the form

$$
0 \stackrel{f_{1}}{\longrightarrow} G_{1} \stackrel{f_{2}}{\longrightarrow} G_{2} \stackrel{f_{3}}{\longrightarrow} G_{3} \stackrel{f_{4}}{\longrightarrow} 0
$$

Corollary B.1.8. Let sequence

$$
0 \stackrel{f_{1}}{\longrightarrow} G_{1} \stackrel{f_{2}}{\longrightarrow} G_{2} \stackrel{f_{3}}{\longrightarrow} G_{3} \stackrel{f_{4}}{\longrightarrow} 0
$$

be exact, then $f_{1}$ is a monomorphism and $f_{2}$ is an epimorphism.

Definition B.1.9. We say that a short exact sequence

$$
0 \stackrel{f_{1}}{\longrightarrow} G_{1} \stackrel{f_{2}}{\longrightarrow} G_{2} \stackrel{f_{3}}{\longrightarrow} G_{3} \stackrel{f_{4}}{\longrightarrow} 0 .
$$

is a split exact sequence if one of the following conditions holds:

1. There exists a group homomorphism $s: G_{3} \rightarrow G_{2}$, called section, such that $f_{3} \circ s=1_{G_{3}}$. 
2. there exists a group homomorphism $r: G_{2} \rightarrow G_{1}$, called retraction, such that $r \circ f_{2}=1_{G_{1}}$.

3. There is an isomorphism $\varphi: G_{2} \rightarrow G_{1} \oplus G_{3}$, such that $\varphi^{-1} \circ r$ is the canonical injection of $G_{1}$ into coproduct $G_{1} \oplus G_{3}$ and $s \circ \varphi$ is a canonical projection of the product $G_{1} \oplus G_{3}$.

Lemma B.1.10 ([42, Proposition 4.3]). All the conditions in the Definition B.1.9 are equivalent.

\section{B.2 Near-rings, skew braces and braces}

For more on near-rings, see [43]. A good introduction into braces one can find in [28].

Definition B.2.1. A near-ring is a set $N$ with two associative binary operations,$+ \cdot$, such that $(N,+)$ is a group and, for all $n, m, m^{\prime} \in N$,

$$
n\left(m+m^{\prime}\right)=n m+n m^{\prime}
$$

If $N$ has an element $1 \in N$, called an identity, such that for all $n \in N 1 n=n=n 1$, then $N$ is called a unital near-ring.

Definition B.2.2. Analogously to the case of rings a near-field is a near-ring such that $(N \backslash\{0\}, \cdot)$ is a group, where 0 is the neutral element for + .

Definition B.2.3. A homomorphism of near-rings is a function $f: N \rightarrow N^{\prime}$ that commutes with both near-ring operations, that is, for all $a, b \in N$,

$$
f(a b)=f(a) f(b) \quad \& \quad f(a+b)=f(a)+f(b)
$$

Moreover, if $N, N^{\prime}$ are unital near-rings, then $f$ is called a homomorphism of unital near-rings if it preserves identities.

Definition B.2.4. A near-ring $N$ is called a ring, if $(N,+)$ is an Abelian group and for all $m, n, m^{\prime} \in N$,

$$
\left(m+m^{\prime}\right) n=m n+m^{\prime} n .
$$


Definition B.2.5. A ring homomorphism is a homomorphism of near-rings between rings. Analogously, a homomorphism of unital rings is a homomorphism of near-rings between unital rings that preserves identity.

Definition B.2.6. Let $R$ be a ring. An element $a \in R$ is called left (right) regular if for all $b \in R$,

$$
a b=0 \Longrightarrow b=0 \quad(b a=0 \Longrightarrow b=0) .
$$

An element is regular if it is both left and right regular.

Definition B.2.7. A domain is a ring $R$ such that for all $a, b \in R$,

$$
a b=0 \Longrightarrow a=0 \text { or } b=0 .
$$

Equivalently, for all $a, b, c \in R$,

$$
(a b=a c \Longrightarrow b=c) \text { and }(c a=b a \Longrightarrow c=b) \text {. }
$$

Also, a domain is a ring such that all non-zero elements are regular.

Definition B.2.8. An Ideal of a ring $R$ is a normal subgroup $I$ of an additive group of $R$ such that for all $a \in I$ and $r \in R$,

$$
r a \in I \quad \& \quad a r \in I .
$$

If only the first (second) membership holds, we say that $I$ is a left (right) ideal. We write $I \triangleleft R$, when $I$ is an ideal of $R$.

Lemma B.2.9. Let $I$ be an ideal of a ring $R$. Then $R / I$ is a ring and a canonical map $\pi: R \rightarrow R / I$ is a ring homomorphism.

Definition B.2.10. Let $R$ be a ring. An ideal $I \triangleleft R$ is essential if for all non-zero ideals $J \triangleleft S, I \cap J \neq\{0\}$.

Definition B.2.11. An essential extension of a $\operatorname{ring} S$ is a ring $R$ such that there exists an injective ring homomorphism $i: S \rightarrow R$, and $i(S)$ is an essential ideal in $R$. 
Definition B.2.12 ([36, K.I. Beidar]). A ring $R$ is said to be a maximal essential extension of a ring $S$, if $S$ is an essential ideal of $R$, and for any ring $A$ which contains $S$ as an ideal, there exists a ring homomorphism $h: A \rightarrow R$ such that $h(s)=s$ for all $s \in S$.

Definition B.2.13. A completely prime ideal of a $\operatorname{ring} R$ is an ideal $P$ such that for all $a, b \in R$,

$$
a b \in P \Longrightarrow a \in P \text { or } b \in P \text {. }
$$

Lemma B.2.14. If $P$ is a completely prime ideal of ring $R$, then $R / P$ is a domain.

Definition B.2.15. Let $R$ be a $\operatorname{ring}$ and $G$ be a group. A group ring is a ring

$$
R G:=\{f: R \rightarrow G \mid \exists S \subseteq G \text { s.t. }|S|<\infty \& \forall x \in G \backslash S f(x)=0\}
$$

with addition and multiplication given for all $f, g \in R G$ by

$$
f+g(x)=f(x)+g(x) \quad f \cdot g(x)=\sum_{u v=x} f(u) g(v) .
$$

Definition B.2.16. Let $R$ be a unital near-ring. The set

$$
U(R):=\left\{u \in R \mid \exists u^{-1} \in R \quad u u^{-1}=u^{-1} u=1\right\}
$$

is called the set of units and an element of $U(R)$ is called a unit.

Remark B.2.17. A set $U(R)$, for any unital near-ring $R$, is a group.

Definition B.2.18. A field is a ring which is also a near-field.

Remark B.2.19. A ring $R$ is a field if and only if $R \backslash U(R)=\{0\}$, where 0 is a neutral element of the additive group of $R$.

Definition B.2.20. The order of an element $u \in U(R)$ of a ring $R$ is the smallest number $n \in \mathbb{N}$ such that $u^{n}=1$.

Definition B.2.21. The characteristic of a $\operatorname{ring} R$ is a smallest number $n$ such that $n \cdot 1=0$. If the number does not exists we say that ring has characteristic zero. 
Definition B.2.22. Let $R$ be a ring. An $R$-module is an Abelian group $G$ together with a ring homomorphism $\varphi: R \rightarrow \operatorname{End}(G)$, i.e. it is an Abelian group together with an $R$-action $\cdot: R \times G \rightarrow G,(r, g) \mapsto r g$ such that for all $r, s \in R$ and $g, h \in G$,

$$
(r s) g=r(s g), \quad(r+s) g=r g+s g \quad \& \quad r(g+h)=r g+r h .
$$

Definition B.2.23. An $R$-module homomorphism or $R$-linear map is a group homomorphism $f: M \rightarrow N$, between $R$-modules $M, N$, such that for all $m \in M$ and $r \in R$,

$$
r f(m)=f(r m) .
$$

Definition B.2.24. Let $M, N, P$ be $R$-modules. Then we say that $P$ is projective if for every surjective $R$-linear map $\pi: M \longrightarrow N$ and every $R$-linear map $f: P \longrightarrow N$ there exists a (not necessarily unique) $R$-linear map $\tilde{f}: P \longrightarrow M$ such that $\pi \circ \tilde{f}=f$. Diagrammatically,

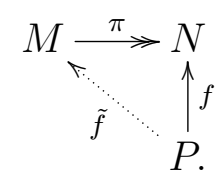

Lemma B.2.25. An R-module $P$ is projective if and only if functor $\operatorname{Hom}_{R}(P,-)$ preserves epimorphisms.

We will briefly introduce skew braces.

Definition B.2.26. A skew brace or a skew left brace is a set $B$ with two binary operations $+, \cdot: B \times B \rightarrow B$ such that $(B,+),(B, \cdot)$ are groups and for all $a, b, c \in B$ the following distributivity holds

$$
a(b+c)=a b-a+a c,
$$

where $-a$ is an inverse in respect to the group operation + , not necessarily Abelian! A skew brace in which $(B,+)$ is an Abelian group is called a left brace. A left brace in which analogous right distributivity rule holds is called a two-sided brace.

Remark B.2.27. In a skew brace $B$ both neutral elements are equal. Let 0 be a neutral element for + and 1 be a neutral element for $\cdot$, then $1=1 \cdot 1=1 \cdot(1+0)=1-1+0=0$. 
Definition B.2.28. A skew brace homomorphism is a map between skew braces that preserves both binary operations.

Definition B.2.29. An ideal of a skew brace $B$ is a normal subgroup $S$ of an additive group of $B$, for all $a \in B a S=S a$ and for any $b \in B, a b-a \in S$.

Proposition B.2.30 ([27, Lemma 2.3]). If $S$ is an ideal of $B$ then $B / S$ is a skew brace, and a canonical map $\pi: B \rightarrow B / S$ is a homomorphism of skew braces.

Proof. The fact that $B / S$ is a skew brace follows by [27, Lemma 2.3]. The second statement follows by the fact that $S$ is a normal subgroup for both groups $(B,+)$ and $(B, \cdot)$, so $\pi$ is a canonical map that is a homomorphism for both groups $(B / S,+)$ and $(B / S, \cdot)$, and therefore a homomorphism of skew braces.

Definition B.2.31. Let $B$ be a skew brace. A socle of $B$ is a set

$$
\operatorname{Soc}(B):=\{a \in B \mid a+b=a b, b+b a=a b+b \text { for all } b \in B\}
$$

If $B$ is a two-sided brace then

$$
\operatorname{Soc}(B):=\{a \in B \mid a+b=a b \text { for all } b \in B\}
$$

Lemma B.2.32 ([27, Lemma 2.5]). A socle of a skew brace $B$ is an ideal.

Proposition B.2.33 ([28, Proposition 3]). If B is a finite non-trivial two-sided brace, then $\operatorname{Soc}(B) \neq\{1\}$. 


\section{Appendix C}

\section{Universal algebra}

This appendix is devoted to universal algebras and introduce the reader to the basics of those algebras. For more on universal algebra, check [18] and [17]. In this part, we will omit the adjective universal, but one should bear in mind that when we say an algebra, we mean a universal algebra.

Definition C.0.1. Let $A$ be a non-empty set and $n$ a nonnegative integer. An n-ary operation on $A$ is a function $f: A^{n} \rightarrow A$, where for $n=0, A^{0}=\{\emptyset\}$ is a singleton set. A number $n$ is called the arity of $f$. We call $f$ a nullary, unary, binary or ternary operation if its arity is $0,1,2$ or 3 , respectively. If we do not specify the arity of $f$ we call it a finitary operation.

Definition C.0.2. A language of algebras is a set $\mathcal{F}$ of functions such that a positive integer $n$ is assigned to each member $f$ of $\mathcal{F}$. This integer is called the arity of $f$. The subset of $n$-ary operations in $\mathcal{F}$ is denoted by $\mathcal{F}_{n}$.

Definition C.0.3. If $\mathcal{F}$ is a language of algebras then an algebra $A$ of type $\mathcal{F}$ is an ordered pair $(A, F)$, where $A$ is a nonempty set and $F$ is a family of finitary operations on $A$ indexed by the language $\mathcal{F}$ such that corresponding to each $n$-ary function symbol $f$ in $\mathcal{F}$ there is an $n$-ary operation $f^{A}$ on $A$. If $F$ is finite, let us say it has $k$ elements, then we write $\left(A, f_{1}, \ldots f_{k}\right)$ instead of $(A, F)$.

Example C.0.4. A group $G$ is an algebra $\left(G, \cdot,^{-1}, 1\right)$, where $\cdot$ is a binary operation,

${ }^{-1}$ is a unary operation and 1 is a nullary operation such that for all $x, y, z \in G$, the 
following holds:

(1) $(x \cdot y) \cdot z=x \cdot(y \cdot z)$,

(2) $x \cdot 1=1 \cdot x=x$

(3) $x \cdot x^{-1}=x^{-1} \cdot x=1$.

Example C.0.5. A ring $R$ is an algebra $(R, \cdot,+,-, 0)$, where $\cdot$ and + are binary operations, - is a unary operation and 0 is a nullary operation, which has the following properties:

(1) $(R,+,-, 0)$ is a group,

(2) $x+y=y+x$

(3) $(x \cdot y) \cdot z=x \cdot(y \cdot z)$

(4) $x \cdot(y+z)=(x \cdot y)+(x \cdot z)$,

(5) $(y+z) \cdot x=(y \cdot x)+(z \cdot x)$,

for all $x, y, z \in R$.

Observe that a field is a quintuple $\left(\mathbb{F}, \cdot,+,{ }^{-1},-, 1,0\right)$ that consists two binary, unary and nullary operations. On the other hand, the brace is a quadruple $\left(B, \cdot,+,{ }^{-1},-, 1\right)$ with one nullary operation less.

Definition C.0.6. Let $A$ and $B$ be two algebras of the same language $\mathcal{F}$. A mapping $\alpha: A \rightarrow B$ is called a homomorphism if

$$
\alpha\left(f^{A}\left(a_{1}, \ldots, a_{n}\right)\right)=f^{B}\left(\alpha\left(a_{1}\right), \ldots, \alpha\left(a_{n}\right)\right)
$$

for each $n$-ary operation $f$ in $\mathcal{F}$ and all $a_{i} \in A$.

If a homomorphism of algebras $\alpha$ is surjective, injective or bijective, then $\alpha$ is an epimorphism, a monomorphism or an isomorphism, respectively. Of course, not every epimorphism is surjective. See Definition A.0.6. 
Definition C.0.7. Let $A$ be an algebra with a language $\mathcal{F}$ and $\sim_{\Theta}$ be an equivalence class on $A$. Then $\sim_{\Theta}$ is a congruence on $A$ if for all $n$-ary functions $f \in \mathcal{F}$ and elements $a_{i}, b_{i} \in A$, if $a_{i} \sim_{\Theta} b_{i}$ holds for $1 \leq i \leq n$ then

$$
f^{A}\left(a_{1}, \ldots, a_{n}\right) \sim_{\Theta} f^{A}\left(b_{1}, \ldots, b_{n}\right)
$$

holds.

Definition C.0.8. Let $\sim_{\Theta}$ be a congruence on an algebra $A$. Then the quotient algebra of $A$ by $\sim_{\Theta}$, denoted by $A / \sim_{\Theta}$ is the algebra whose underlying set is $A / \sim_{\Theta}$ and finitary operations satisfy

$$
f^{A / \sim_{\Theta}}\left(a_{1} / \sim_{\Theta}, \ldots, a_{n} / \sim_{\Theta}\right)=f^{A}\left(a_{1}, \ldots, a_{n}\right) / \sim_{\Theta},
$$

where $a_{i} \in A$ and $f$ is an $n$-ary function in $\mathcal{F}$.

Theorem C.0.9. (see [18], Definition 6.7 and Theorem 6.8) Let $\alpha: A \rightarrow B$ be a homomorphism of algebras with language $\mathcal{F}$. Then the kernel of $\alpha$, defined by

$$
\operatorname{Ker}(\alpha)=\left\{(a, b) \in A^{2} \mid \alpha(a)=\alpha(b)\right\}
$$

is a congruence on $A$. Moreover canonical map $\pi: A \rightarrow A / \operatorname{Ker}(\alpha)$ is an epimorphism.

Theorem C.0.10 (Homomorphism theorem). (see [18], Theorem 6.12) Let $\alpha: A \rightarrow B$ be a surjective homomorphism. Then $B$ is isomorphic with $A / \operatorname{Ker}(\alpha)$.

Theorem C.0.11 (Second isomorphism theorem). (see [18], Theorem 6.15) If $\sim_{\phi}, \sim_{\Theta}$ are congruences on $A$ such that $\sim_{\phi} \subset \sim_{\Theta}$, then an algebra $A / \sim_{\phi}$ is isomorphic to $\left(A / \sim_{\phi}\right) /\left(\sim_{\Theta} / \sim_{\phi}\right)$.

Remark C.0.12. Algebras with the same language $\mathcal{F}$ form a category with homomorphisms of algebras as morphisms. We will denote this category by $\mathcal{F}$-Alg.

Proposition C.0.13 ([17], Proposition 9.1.6). Let $\mathcal{F}$ be a language. Then the category $\mathcal{F}$-Alg has small limits, which can be constructed by taking the limits of the underlying sets and making them $\mathcal{F}$-algebras under pointwise operations. 
Theorem C.0.14 ([17], Theorem 9.3.8). The category $\mathcal{F}$-Alg has all small colimits.

Definition C.0.15. (see [17], Definition 9.4.6) A variety of $\mathcal{F}$-Alg is a full subcategory $\mathfrak{K}$ of $\mathcal{F}$-Alg for which objects are algebras which satisfies some set of identities $I$ and every object in $\mathcal{F}$-Alg has an underlying structure of a set. A category is called a variety of algebras if it is a variety of $\mathcal{F}$-Alg for some language $\mathcal{F}$.

Theorem C.0.16. (see, [17], Theorem 9.4.14) If $\mathfrak{K}$ is a variety of $\mathcal{F}$-Alg. then $\mathfrak{K}$ has small colimits, objects presented by generators and relations, and free objects on all small sets.

The most common examples of the varieties are a category of groups Grp, a category of rings, denoted by Ring, and a category of modules over a ring $R$ denoted by $R$-mod. 


\section{Bibliography}

[1] H. Prüfer, Theorie der Abelschen Gruppen. I. Grundeigenschaften, Math. Z. 20 (1924), 165-187.

[2] R. Baer, Zur Einführung des Scharbegriffs, J. Reine Angew. Math. 160 (1929), 199-207.

[3] A.K. Suškevič, Theory of Generalized Groups, Goc. Naučno-Techn. Izdat. Ukrainy, Kharkov, 1937.

[4] J. Certaine, The ternary operation $(a b c)=a b^{-1} c$ of a group, Bull. Amer. Math. Soc., 49 (1943), 869-877.

[5] W. Rump, Braces, radical rings, and the quantum Yang-Baxter equation, J. Algebra 307 (2007), 153-170.

[6] S. Majid, Foundations of quantum group theory, Cambridge University Press, 1995.

[7] A. Smoktunowicz, A note on set-theoretic solutions of the Yang-Baxter equation, J. Algebra 500 (2018), 3-18.

[8] A. Smoktunowicz \& L. Vendramin, On skew braces (with an appendix by N. Byott and L. Vendramin), J. Comb. Algebra, 2 (2018), 47-86.

[9] A. Soloviev, Non-unitary set-theoretical solutions to the quantum Yang-Baxter equation, Math. Res. Lett. 7 (2000), 577-596.

[10] F. Cedó, T. Gateva-Ivanova, A. Smoktunowicz, On the Yang-Baxter equation and left nilpotent left braces, J. Pure Appl. Algebra 221 (2017), 751-756. 
[11] J.-H. Lu, M. Yan \& Y.-C. Zhu, On the set-theoretical Yang-Baxter equations, Duke Math. J. 104 (2000), 1-18.

[12] L. Vendramin, Problems on skew left braces, Adv. Group Theory Appl., 7 (2019), 15-37.

[13] P. Etingof, T. Schedler \& A. Soloviov, Set-theoretical solutions to the quantum Yang-Baxter equation, Duke Math. J. 100 (1999), 169-209.

[14] W. Rump, Set-theoretic solutions to the Yang-Baxter equation, skew-braces, and related near-rings, J. Algebra Appl. 18 (2019), 1950145.

[15] T. Brzeziński, Trusses: Between braces and rings, Trans. Amer. Math. Soc. 372 (2019), 4149-4176.

[16] T. Brzeziński, Trusses: Paragons, ideals and modules, J. Pure Appl. Algebra 224 (2020), 106258.

[17] G.M. Bergman, An Invitation to General Algebra and Universal Constructions, Springer Cham, 2015.

[18] S. Burris \& H.P. Sankappanavar, A Course in Universal Algebra, Springer-Verlag, The Millenium Edition (on-line), http://math.hawaii.edu/ ralph/Classes/619/univalgebra.pdf.

[19] T. Brzeziński \& B. Rybołowicz, Modules over trusses vs modules over rings: direct sums and free modules, Algebras and Representation Theory (2020).

[20] C.D. Holdings \& M.V. Lawson, Wagner's Theory of Generalised Heaps, Springer Berlin 2017.

[21] W.A. Dudek, Ternary quasigroups connected with the affine geometry, Algebras, Groups and Geometries, 16 (1999), 329-354.

[22] B. Rybołowicz, A note on a free group. The decomposition of a free group functor through the category of heaps, Preprint, arXiv:2101.04752 (2021). 
[23] O. Schreier, Die Untergruppen der Freien Gruppen,Abh. Math. Sem. Univ. Hamburg 5, 161-183 (1927).

[24] S. Mac Lane, Categories for the Working Mathematician (Second ed.), Springer, New York, 1998.

[25] M. Barr \& Ch. Wells, Toposes, Triples and Theories, Reprints in Theory and Applications of Categories, 12 (2005), 1-287.

[26] T. Brzeziński, B. Rybołowicz \& S. Mereta, From pre-trusses to skew braces, Accepted, Publicacions Matemàtiques, arXiv: 1909.05807 (2019).

[27] L. Guarnieri \& L. Vendramin, Skew braces and the Yang-Baxter equation, Math. Comp. 86 (2017), 2519-2534.

[28] F. Cedó, E. Jespers \& J. Okniński, Braces and the Yang-Baxter equation, Commun. Math. Phys. 327 (2014), 101-116.

[29] W. Rump, Classification of cyclic braces, J. Pure Appl. Algebra 209 (2007), 671685.

[30] O. Ore, Linear equations in noncommutative fields, Ann. Math. 32 (1931), 463-477. Available online: https://www.jstor.org/stable/1968245.

[31] Z. Škoda, Noncommutative localization in noncommutative geometry, Lond.Math.Soc.Lect.Note Ser. 330 (2006), 220-313, arXiv:math/0403276v2.

[32] T. Brzeziński \& B. Rybołowicz, On congruence classes and extensions of rings with an application to braces, Communications in Contemporary Mathematics, 23 (2021), No. 4, 2050010.

[33] T. Brzeziński, B. Rybołowicz \& P. Saracco, On functors between categories of modules over trusses, Preprint, arXiv:2006.16624 (2020).

[34] R. R. Andruszkiewicz, T. Brzeziński, \& B. Rybołowicz, Ideal ring extensions and trusses, Preprint, arXiv:2101.09484 (2021). 
[35] J.L. Dorroh, Concerning adjunctions to algebras, Bull. Amer. Math. Soc., 38 (1932), 85-88.

[36] K.I. Beidar, On essential extensions, maximal essential extensions and iterated maximal essential extensions in radical theory, [in:] Theory of Radicals (Szekszard, 1991), Colloq. Math. Soc. Janós Bolyai 61, North-Holland, Amsterdam 1993, pp. $17-26$.

[37] G. M. Kelly, Basic concepts of enriched category theory. Reprint of the 1982 original. Repr. Theory Appl. Categ., No. 10 (2005).

[38] B. Mitchell, Rings with several objects, Adv. Math., 8 (1972), 1-161.

[39] F. Borceux, Handbook of categorical algebra. 2. Categories and structures. Encyclopedia of Mathematics and its Applications, 51. Cambridge University Press, Cambridge, 1994.

[40] T. Leinster, Basic Category Theory, Cambridge Studies in Advanced Mathematics, 143, Cambridge University Press, arXiv:1612.09375, 2014.

[41] C. E. Linderholm, A Group Epimorphism is Surjective, The American Mathematical Monthly 77 (1970), 176-177.

[42] S. Mac Lane, Homology, Springer-Verlag Berlin, 1963. Available online https://www.maths.ed.ac.uk/ṽ1ranick/papers/maclane_homology.pdf

[43] G. Pilz, Near-rings. The Theory and Its Applications, 2nd edition. North-Holland Mathematics Studies, 23. North-Holland Publishing Co., Amsterdam, 1983. 